\title{
WestVirginiaUniversity
}

THE RESEARCH REPOSITORY @ WVU

Graduate Theses, Dissertations, and Problem Reports

2006

\section{Cracking studies of high-performance concrete for bridge decks}

\author{
Zhiguo Gong \\ West Virginia University
}

Follow this and additional works at: https://researchrepository.wvu.edu/etd

\section{Recommended Citation}

Gong, Zhiguo, "Cracking studies of high-performance concrete for bridge decks" (2006). Graduate Theses, Dissertations, and Problem Reports. 4229.

https://researchrepository.wvu.edu/etd/4229

This Dissertation is protected by copyright and/or related rights. It has been brought to you by the The Research Repository @ WVU with permission from the rights-holder(s). You are free to use this Dissertation in any way that is permitted by the copyright and related rights legislation that applies to your use. For other uses you must obtain permission from the rights-holder(s) directly, unless additional rights are indicated by a Creative Commons license in the record and/ or on the work itself. This Dissertation has been accepted for inclusion in WVU Graduate Theses, Dissertations, and Problem Reports collection by an authorized administrator of The Research Repository @ WVU.

For more information, please contact researchrepository@mail.wvu.edu. 


\title{
Cracking Studies of High-Performance Concrete \\ for Bridge Decks
}

\author{
by \\ Zhiguo Gong
}

\begin{abstract}
Dissertation submitted to the
College of Engineering and Mineral Resources

at West Virginia University

in partial fulfillment of the requirements

for the Degree of
\end{abstract}

\author{
Doctor of Philosophy \\ in \\ Civil Engineering
}

\author{
Karl E. Barth, Ph.D. \\ Julio F. Davalos, Ph.D., Co-chair \\ Xingbo Liu, Ph.D. \\ Sam Mukdadi, Ph.D. \\ Indrajit Ray, Ph.D., Chair
}
Department of Civil and Environmental Engineering
Morgantown, West Virginia 2006

Keywords: Cracking, High-Performance Concrete, Bridge Deck, Shrinkage, Fracture Mechanics

Copyright @2006 Zhiguo Gong 


\title{
Cracking Study of High-Performance Concrete for Bridge Decks
}

\author{
Zhiguo Gong \\ Advisor: Dr. Indrajit Ray, and Dr. Julio F. Davalos
}

\begin{abstract}
:
The cracking problem has been one of the major concerns for any concrete bridge deck, especially for cast-in-place concrete decks. The cracking can accelerate deterioration of the structure, thereby increasing maintenance costs and potentially shortening the system service-life. In order to achieve low permeability and reasonable strength, HPC deck mixture designs usually include a higher cementitious material content, which means more shrinkage, and therefore, makes the bridge deck more susceptible to cracking. Thus, further and better understanding of shrinkage and cracking properties of HPC designated for bridge decks is necessary. As part of a comprehensive project, this dissertation is focused on the restrained shrinkage and cracking study of HPC for bridge decks.
\end{abstract}

A total of 24 HPC mixtures were developed, and tested. The relationships among compressive strength, modulus of elasticity, and direct tensile strength were established. Free shrinkage and restrained shrinkage tests were conducted for selected HPC mixtures. The AASHTO ring specimen test was adopted for the restrained shrinkage study. Based on the results, the relationship among basic material parameters (such as 28-day compressive strength, modulus of elasticity, and 90-day free shrinkage) versus cracking onset days was established. The concept of cracking index was proposed as a convenient way to evaluate the cracking properties of different HPC mixtures. This new concept was validated with actual field data for bridges in WV. A threshold cracking onset day was proposed as the criterion for any acceptable HPC mix designs.

A fracture mechanics method by a combined experimental and FE modeling approach was developed for predicting cracking for the AASHTO ring test. The predicted cracking onset day was in good agreement with the experimental results, and the AASHTO ring test was validated as an effective method for cracking evaluation of HPC mixtures. Finally, comprehensive fracture mechanics tests were conducted for selected HPC mixtures, and a data set for fracture mechanics properties was established as a referance for future studies. 


\section{ACKNOWLEDGMENTS}

First of all, I want to express my sincere and deep gratitude to Dr. Julio F. Davalos for his continuing support, guidance, understanding and encouragement during last four years. My sincere appreciation also goes to Dr Indrajit Ray for his tremendous support and valuable suggestions. His guidance was invaluable at every step of this work.

Special thanks are given to the committee members: Dr. Karl E. Barth, Dr. Xingbo Liu, and Dr. Sam Mukdadi for their interests in my research and careful evaluation of this dissertation.

I would like to thank Mr. Justin Robinson, Mr. David Turner, Mr. Doug Cutlip and Mr. William Comstock for the technical help they have provided in the concrete and structure labs.

Also I want to thank Dayong Fan, Anituddha Chatterjee, Hong Tao, Yi chen, Zhenghua Sun, and An Chen for their generous help.

I gratefully acknowledge the financial support from West Virginia Department of Transportation. I also acknowledge Arrow Concrete Company, Degussa Corp., Advanced Cement Technologies Inc., and other materials suppliers.

With great appreciation, I acknowledge the endless support I received from my wife Yun Sun and my parents. 


\section{TABLE OF CONTENTS}

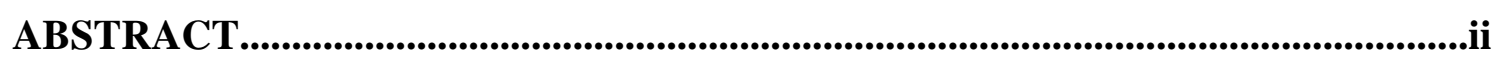

ACKNOWLEDGEMENTS...................................................................................ii

TABLE OF CONTENTS ........................................................................................................ iv

LIST OF TABLES................................................................................................................... ix

LIST OF FIGURES........................................................................................................... $\mathrm{x}$

CHAPTER ONE INTRODUCTION............................................................................. 1

1.1 Background of High-Performance Concrete......................................................... 1

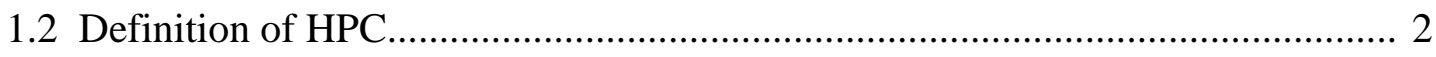

1.3 HPC Bridge Deck and Applications................................................................. 3

1.4 Research Significance.............................................................................

1.5 Goals and Objectives................................................................................. 8

1.6 Organization of the Dissertation........................................................................ 9

CHAPTER TWO LITERATURE REVIEW............................................................. 11

2.1 Cracking Mechanism of Bridge Deck.................................................................. 11

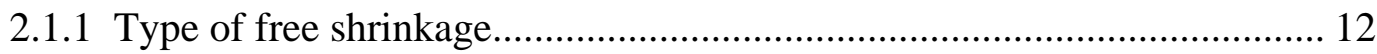

2.1.2 Creep.................................................................................................. 16

2.1.3 Restrained shrinkage in bridge decks........................................................18

2.1.4 Mechanism of cracking.............................................................................. 18

2.2 A Review of Restrained Shrinkage Test Methods............................................... 19

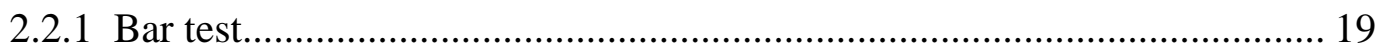

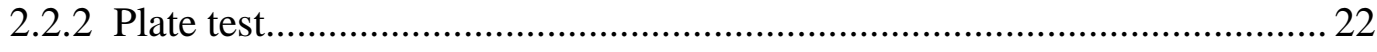

2.2.3 Ring specimen test................................................................................ 24

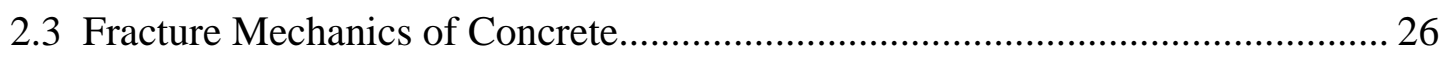

2.3.1 History of fracture mechanics from Griffith approach to cohesive crack

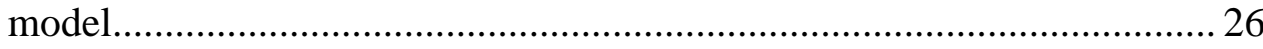

2.3.2 Fracture process zone of concrete............................................................. 28

2.3.3 Fracture theories for concrete....................................... 30 


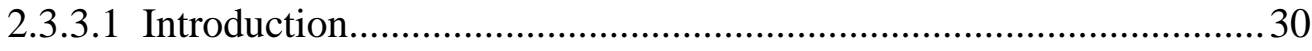

2.3.3.2 Nonlinear cohesive models............................................................... 31

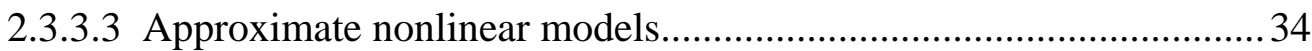

2.4 Test Methods for the Determination of Fracture Parameters..................................39

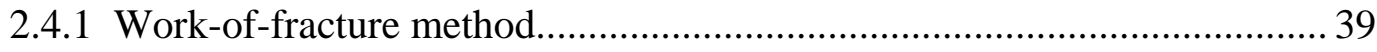

2.4.2 Jenq-Shah method...................................................................................... 41

2.4.3 Size effect method......................................................................................43

CHAPTER THREE METERIALS AND METHODS............................................45

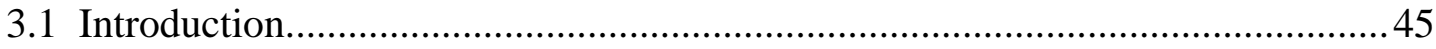

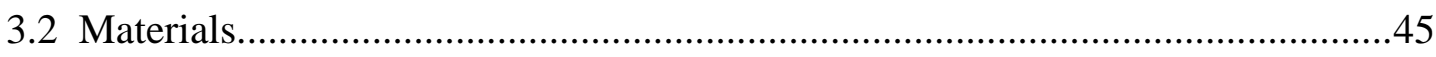

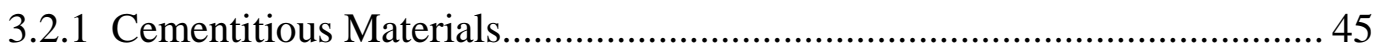

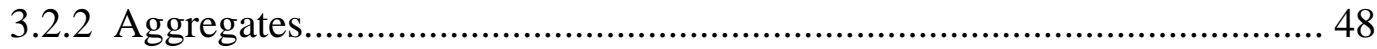

3.2.3 Chemical admixtures................................................................................ 51

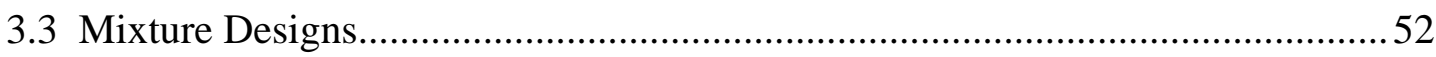

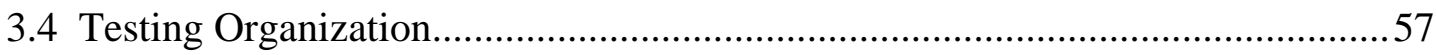

3.4.1 Preparation of Concrete......................................................................... 57

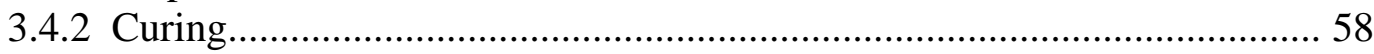

3.4.3 Testing of fresh properties....................................................................... 58

3.4.4 Testing of mechanics properties................................................................. 59

3.4.4.1 Compressive strength test................................................................ 59

3.4.4.2 Modulus of elasticity test..................................................................... 59

3.4.4.3 Direct tensile test.................................................................................60

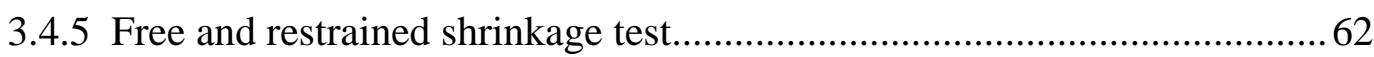

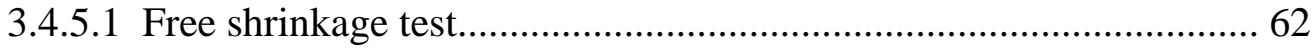

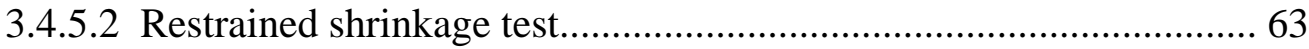

3.4.6 Fracture mechanics test................................................................................... 63

CHAPTER FOUR PROPERTIES OF FRESH AND HARDENED HPC............... 64

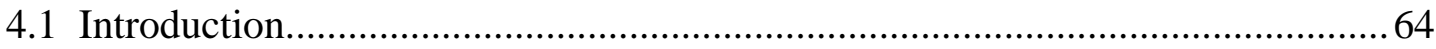

4.2 Fresh Properties............................................................................................64

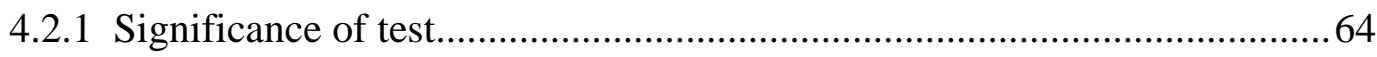

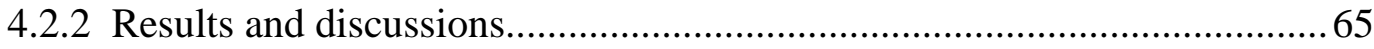

4.3 Compressive Strength and Modulus of Elasticity...................................................66

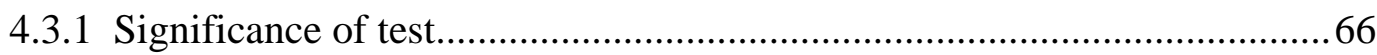

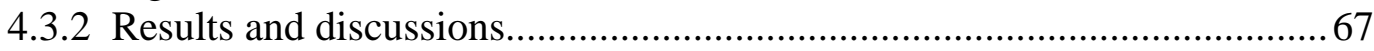

4.3.2.1 Compressive strength........................................................................67

4.3.2.2 Modulus of elasticity and Poisson's ratio........................................... 73 
4.4 Direct Tensile Strength and Modulus of Elasticity in Tension........................... 75

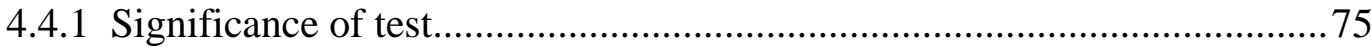

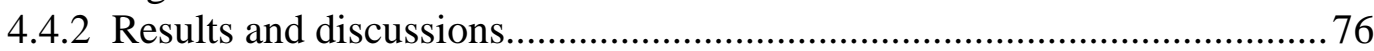

4.4.2.1 Validation of the testing system.................................................... 76

4.4.2.2 Selection of mixtures and failure description................................ 77

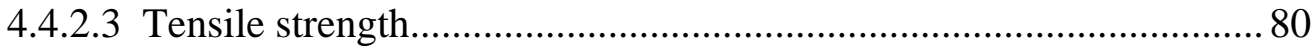

4.4.2.4 Relationship between direct tensile strength and compressive

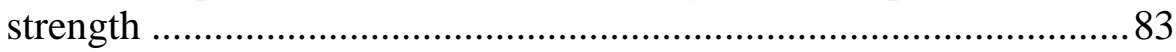

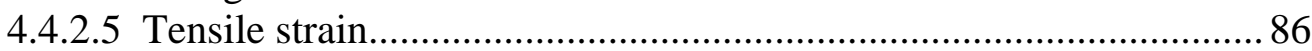

4.4.2.6 Tensile modulus of elasticity................................................... 87

4.5 Free Shrinkage

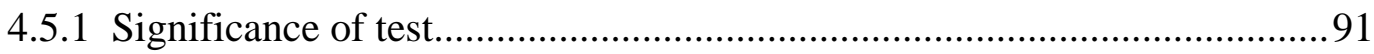

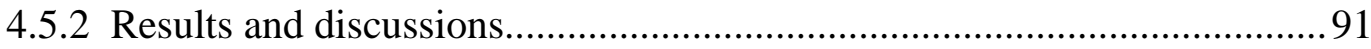

4.5.2.1 Effect of different coarse aggregate............................................. 91

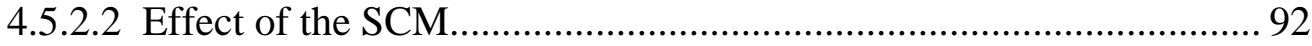

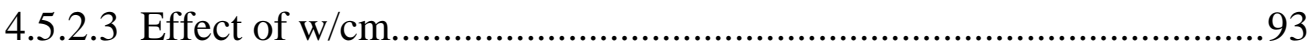

4.5.2.4 Validation of experimental data..................................................95

4.5.3 Prediction of the free shrinkage........................................................... 96

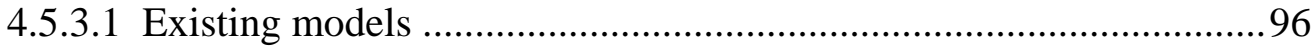

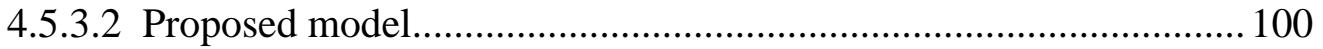

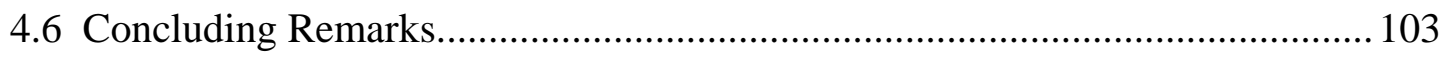

CHAPTER FIVE RESTRAINED SHRINKAGE TEST ................................... 105

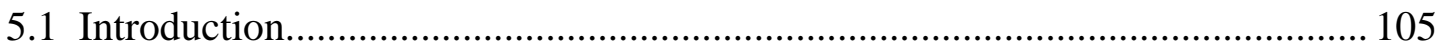

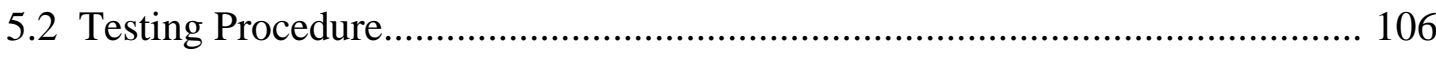

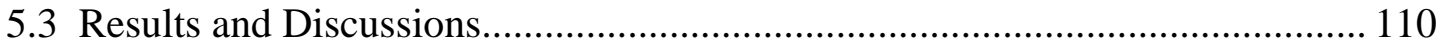

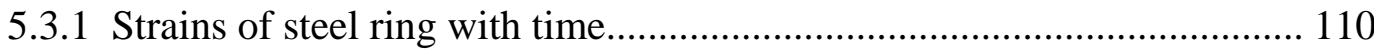

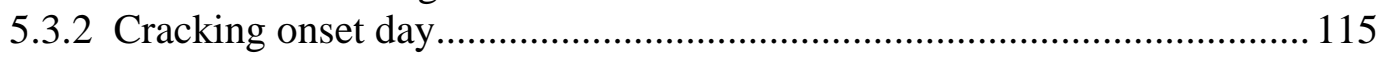

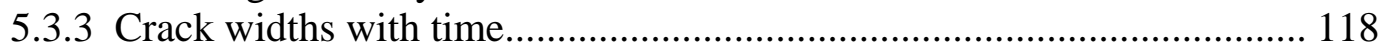

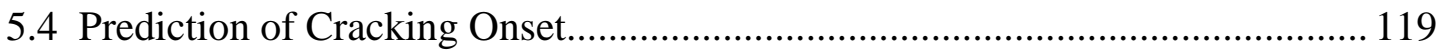

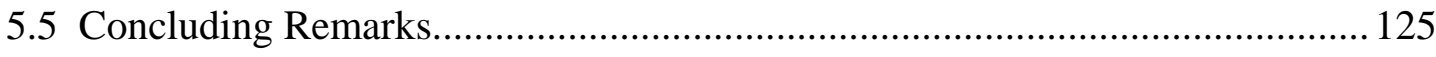

\section{CHAPTER SIX NUMERICAL ANALYSIS OF CRACKING OF RING}

SPECIMENS....................................................................... 126

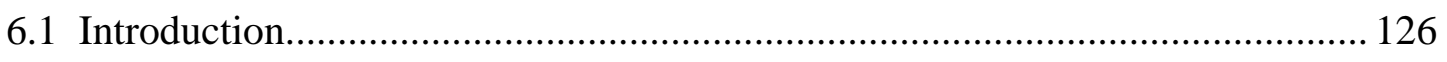

6.2 Failure Criteria Based on Fracture Mechanics............................................... 127

6.3 G-Curves for AASHTO Concrete Ring........................................................ 130 
6.4 R-Curves for Different HPC Mixtures................................................................ 136

6.5 Maximum Allowable Tensile Strains....................................................................143

6.6 Prediction of Cracking Onset............................................................................. 148

6.6.1 Estimation of creep...............................................................................148

6.6.2 Prediction of cracking onset.......................................................................151

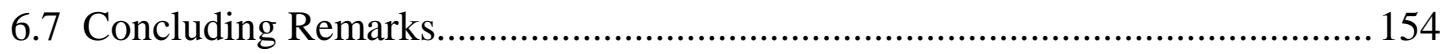

CHAPTER SEVEN FRACTURE MECHANICS TEST......................................... 155

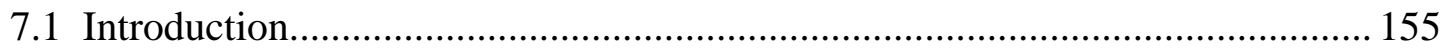

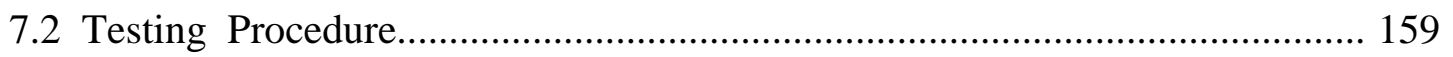

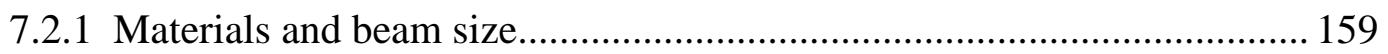

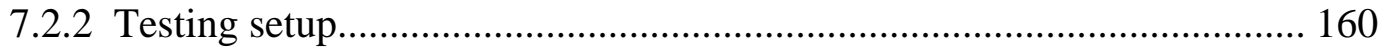

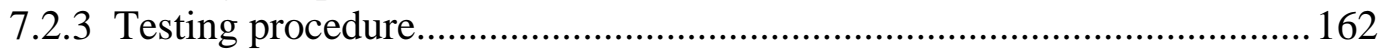

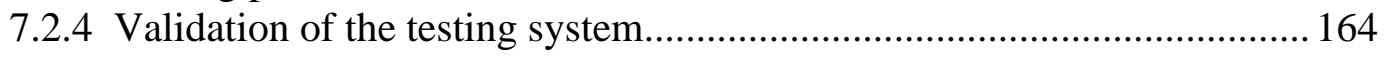

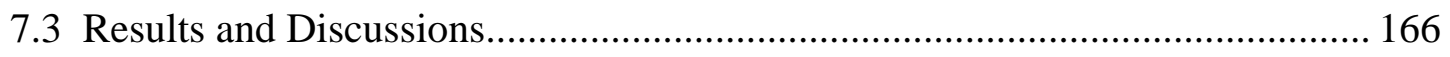

7.3.1 Crack propagation and fracture surface................................................... 166

7.3.2 Determination of the fracture energy of different HPC mixtures............... 169

7.3.3 Comparison of fracture energy for different HPC mixtures................... 175

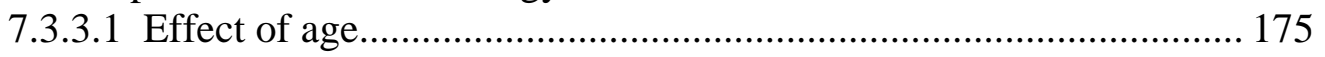

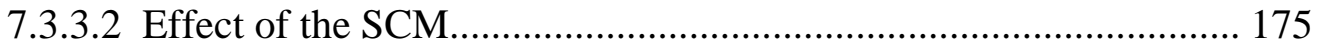

7.3.3.3 Effect of coarse aggregate types.....................................................177

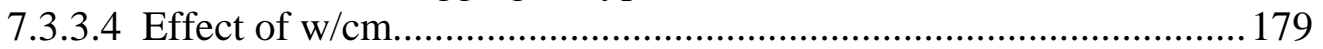

7.3.4 Relationship between fracture energy $G_{F}$ and compressive strength...... 180

7.3.5 Size effect for fracture mechanics test..................................................... 181

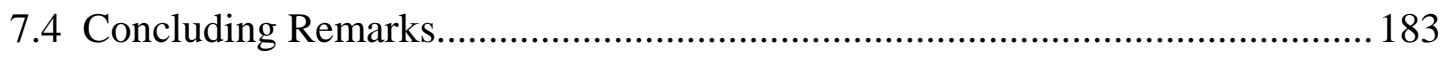

CHAPTER EIGHT CONCLUSIONS AND RECOMMENDATIONS.................. 185

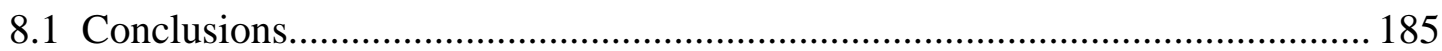

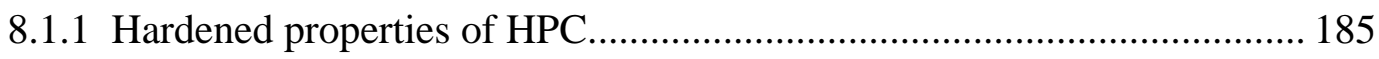

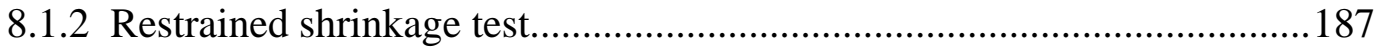

8.1.3 Numerical analysis................................................................................ 188

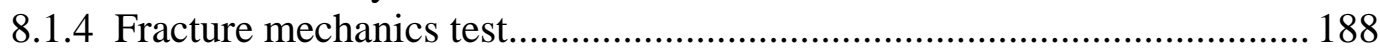

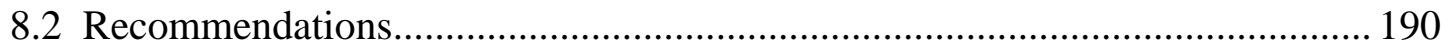


REFERENCES............................................................................................................. 191 APPENDIX A COMPRESSIVE STRENGTH, MODULUS OF ELASTICITY, AND POISSON'S RATIOS DATA................................................ 200

APPENDIX B DIRECT TENSILE STRANGTH DATA ….................................... 202

APPENDIX C FREE SHRINKAGE DATA.....................................................204

APPENDIX D COMPUTING PROGRAMS FOR NUMERICAL ANALYSIS...206

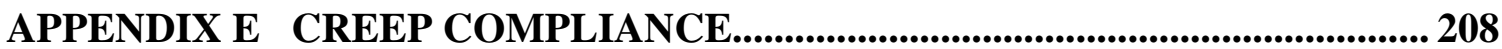




\section{LIST OF TABLES}

Table 2.1 Size of specimen for Hillerborg's test (from RILEM 50-FMC, 1985)........40

Table 2.2 Size of specimen for two parameter test

(from RILEM TC-89-FMT, 1990).

Table 3.1 Basic properties and oxide compositions of cement, silica fume,

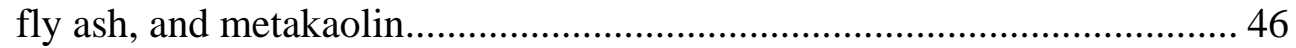

Table 3.2 Basic properties and compound compositions of slag...............................47

Table 3.3 Sieve analysis and basic properties of coarse aggregates.................. 49

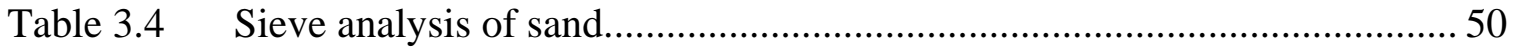

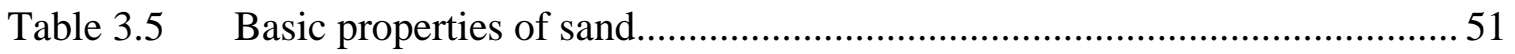

Table 3.6 Overview of various fly ash + silica fume mixtures............................... 53

Table 3.7 Overview of various slag + silica fume mixtures.................................... 53

Table 3.8 Mixture types and percentage of SCM used............................................. 54

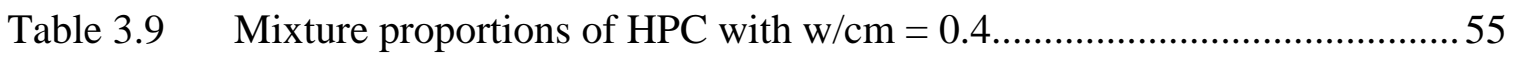

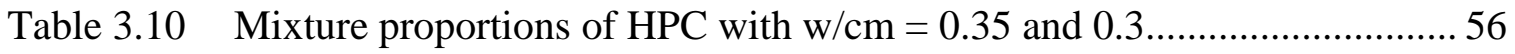

Table 4.1 Total cementitious material and water usage per $\mathrm{m}^{3}$ of different

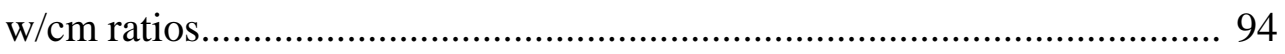

Table 4.2 Values of $f$ and $\varepsilon_{\text {shu }}$ for HPC mixtures with different w/cm.................... 101

Table 5.1 Stresses and strains inside concrete rings.............................................. 112

Table 5.2 Cracking onset days for different HPC mixtures.................................... 115

Table 5.3 Basic material parameters and cracking onset data of all

HPC mixtures tested..................................................................... 120

Table 5.4 Field data from $40^{\text {th }}$ Street and I-64 Dunbar projects................................ 123

Table 6.1 Maximum allowable tensile strains for HPC at different ages..................143

Table 6.2 Comparison of predicted values with measured values............................ 151

Table 7.1 Experimental results of fracture energy for different HPC........................ 172

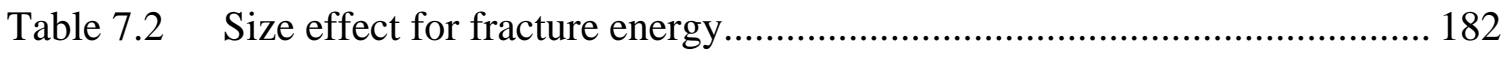




\section{LIST OF FIGURES}

Figure 1.1 FHWA survey map (from Triandafilou, 2004)..................................... 6

Figure 2.1 Transverse cracks on bridge deck....................................................... 11

Figure 2.2 Factors related to the cracking of bridge deck......................... 12

Figure 2.3 Mechanism of autogenous shrinkage (from Bissonnette et al., 2001) ...... 14

Figure 2.4 Mechanism for causes of drying shrinkage of cement paste

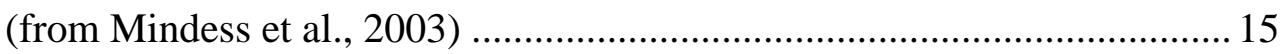

Figure 2.5 Mechanism of restrained shrinkage of bridge deck

(from Saadeghvaziri and Hadidi, 2002) .................................................. 17

Figure 2.6 Mechanism of cracking (from Neville, 1996) ........................................ 19

Figure 2.7 The cracking frame apparatus (from Springenschmid et al., 1994) ......... 20

Figure 2.8 Scheme of restrained shrinkage testing system

(from Igarashi et al., 2000) ................................................................. 21

Figure 2.9 JCI restrained shrinkage test device (from JCI, 1996) ............................ 22

Figure 2.10 Slab test scheme (from Banthia et al., 1996) ....... 23

Figure 2.11 Ring specimen test setup (from Grzyboski and Shah, 1990) ................... 25

Figure 2.12 Barenblatt and Dugdale’s cohesive models........................................... 28

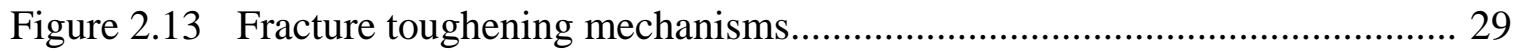

Figure 2.14 Typical stress-strain curve for concrete in tension.................................. 31

Figure 2.15 Hillerborg's fictitious theory (from Hillerborg et al., 1976) ..................... 32

Figure 2.16 Crack bond theory (from Bazant and Oh, 1983) .......................................34

Figure 2.17 Two parameter model (from Jenq and Shah, 1985) .................................35

Figure 2.18 Effective crack model (from Nallathambi and Karihaloo, 1989) .............. 37

Figure 2.19 Hillerborg's three-point bending test (from RILEM 50-FMC, 1985) ....... 40

Figure 2.20 Bazant's size effect method (from RILEM TC-89-FMT, 1990) ................ 42

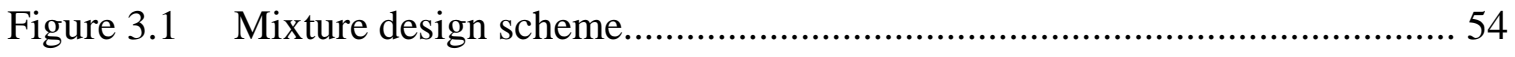

Figure 3.2 Static modulus of elasticity test in progress........................................ 59

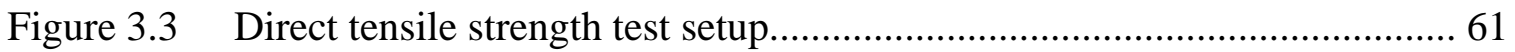

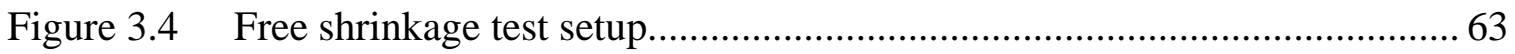


Figure 4.1 Compressive strength with time up to 90-day for 24 HPC mixtures........... 70

Figure 4.2 Effect of SCM on 28-day compressive strength..................................... 71

Figure 4.3 Compressive strength from current study compared to ready mix plant

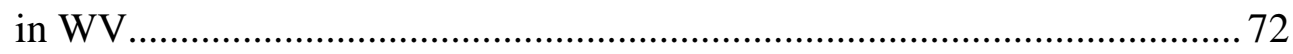

Figure 4.4 Effect of SCM on 28-day modulus of elasticity..................................... 73

Figure 4.5 Relationships between 28-day modulus of elasticity and compressive

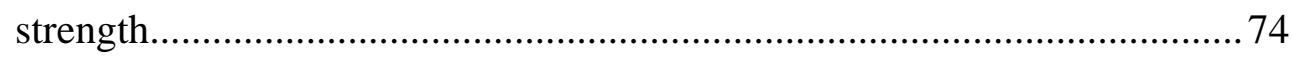

Figure 4.6 Readings from two strain gauges on opposite sides................................ 77

Figure 4.7 Cross-section of broken specimens.................................................... 78

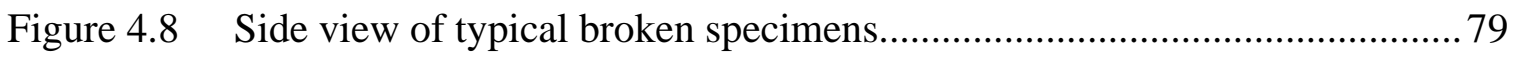

Figure 4.9 Typical stress-strain curves of HPC in tension........................................ 79

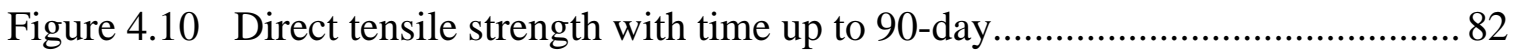

Figure 4.11 Direct tensile strength at 28-day for HPC with different SCM...................83

Figure 4.12 Direct tensile strength with time for HPC with different w/cm.................. 83

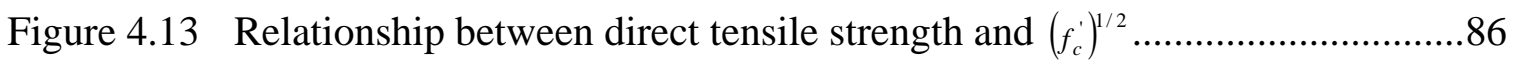

Figure 4.14 Maximum tensile strains of different HPC mixtures................................ 86

Figure 4.15 Method to calculate the tensile modulus of elasticity................................ 88

Figure 4.16 Tensile and compressive moduli of elasticity of various HPC mixtures..... 88

Figure 4.17 Effect of coarse aggregates on tensile modulus of elasticity...................... 89

Figure 4.18 Relationships between tensile modulus of elasticity and tensile strength... 90

Figure 4.19 Effect of coarse aggregates on free shrinkage of HPC............................. 92

Figure 4.20 Effect of SCM on free shrinkage of HPC............................................... 93

Figure 4.21 Effect of $\mathrm{w} / \mathrm{cm}$ ratios on free shrinkage of HPC...................................... 94

Figure 4.22 Comparison of current research data with field data for class H concrete...95

Figure 4.23 Comparison of prediction models and measured free shrinkage................. 99

Figure 4.24 A typical plot of the best-fit curves................................................... 101

Figure 4.25 Comparative plots of proposed prediction models and experimental data..................................................................... 102

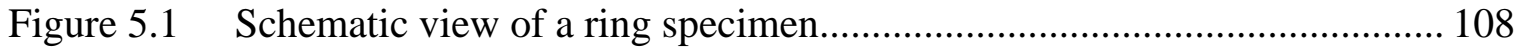

Figure 5.2 Close-view of a typical ring specimen with four strain gages.................. 109 
Figure 5.3 Overview of test setup in an environmental chamber and data

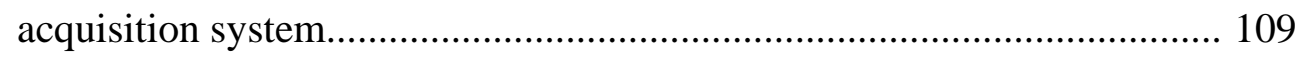

Figure 5.4 Illustration of interaction between concrete ring and steel ring................. 110

Figure 5.5 Strain developments with time inside the steel rings................................... 114

Figure 5.6 Typical cracks on the surface of the concrete rings.................................... 116

Figure 5.7 Comparative plots of cracking onset for different HPC mixtures............... 117

Figure 5.8 The development of crack width with time of different HPC mixtures..... 118

Figure 5.9 Regression analysis of cracking onset day and basic material

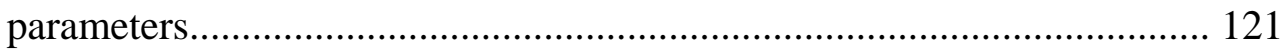

Figure 5.10 Threshold plane of cracking onset based on field data...............................124

Figure 6.1 Failure criteria based on fracture mechanics............................................... 129

Figure 6.2 Simplification of ring specimen for fracture analysis................................ 131

Figure 6.3 2-D mesh for FE model of ring specimen................................................ 132

Figure 6.4 Element type for fracture mechanics analysis (from ANSYS, 2005).........133

Figure 6.5 The geometry function for ring specimen.................................................. 134

Figure 6.6 Typical G-curves for AASHTO ring at different stress levels................... 135

Figure 6.7 Definition of the P-CMOD equivalence (from Elices and Planas, 1993)...136

Figure 6.8 Fracture mechanics test setup and typical P-CMOD response................... 138

Figure 6.9 The P-CMOD responses for HPC mixtures at different ages...................... 141

Figure 6.10 The R-curves for HPC mixtures at different ages....................................... 142

Figure 6.11 Critical crack lengths for SL-SF-L1-0.4 at different ages........................... 145

Figure 6.12 Critical crack lengths for SL-SF-G2-0.4 at different ages......................... 146

Figure 6.13 Critical crack lengths for SL-SF-L1-0.3 at different ages........................... 147

Figure 6.14 Free shrinkage, creep and free shrinkage minus creep development

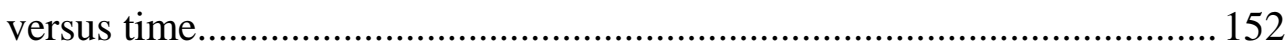

Figure 6.15 Theoretical predictions of cracking onset days for ring specimens.............153

Figure 7.1 Load and deflection responses of ductile and brittle structures.................. 156

Figure 7.2 Size effect of geometrically similar structures with different size (from ACI 446, 1991) ………………………………………………...... 157

Figure 7.3 The sketch of the beam and displacement transducer frame........................ 160

Figure 7.4 The testing frame...................................................................................... 161 


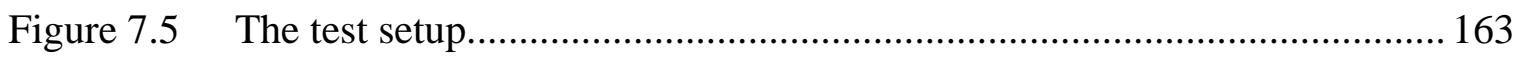

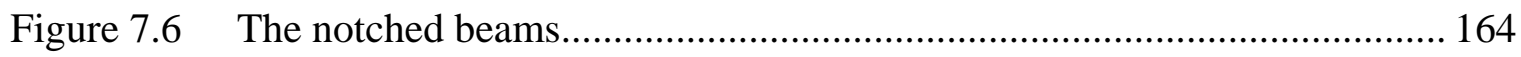

Figure 7.7 Typical load-displacement curves............................................................. 165

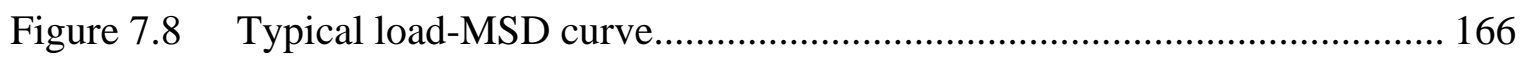

Figure 7.9 Illustration of the crack propagation........................................................ 167

Figure 7.10 Typical crack propagation paths...................................................................167

Figure 7.11 The fracture surface of typical broken specimens........................................ 168

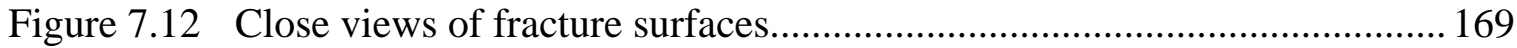

Figure 7.13 Typical load-MSD responses for three replicate specimens....................... 170

Figure 7.14 The method to calculate the fracture energy.............................................. 171

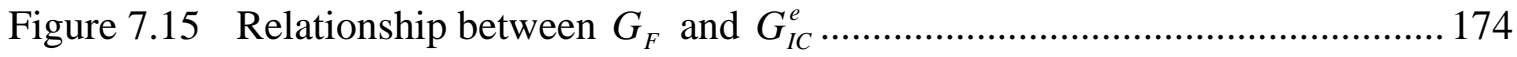

Figure 7.16 Fracture energy with time...........................................................................175

Figure 7.17 Load-MSD curves for HPC mixtures with different SCM........................ 176

Figure 7.18 Comparison of fracture energy of HPC mixtures with different SCM...... 176

Figure 7.19 Load-MSD curves for HPC mixtures with different coarse aggregates.....178

Figure 7.20 Comparison of fracture energy for HPC mixtures with different coarse

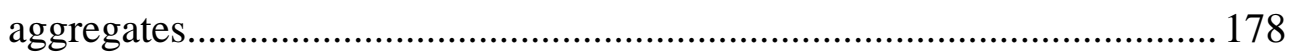

Figure 7.21 Load-MSD curves for HPC mixtures with different w/cm ratios............... 179

Figure 7.22 Comparison of fracture energy for HPC mixtures with different

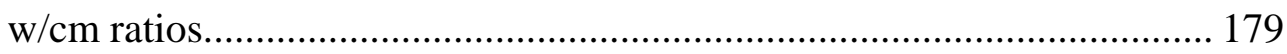

Figure 7.23 Relationship between fracture energy and compressive strength............... 181 


\section{CHAPTER ONE}

\section{INTRODUCTION}

This introductory chapter presents background information on high-performance concrete (HPC) with emphasis on highway bridge deck application. Also, the significance, objectives, and organization of the present research work are discussed.

\subsection{Background on High-Performance Concrete}

HPC first appeared as High-Strength Concrete (HSC). From the early 1960s to 1970s in Chicago area (Freedman, 1971), HSC was developed and applied to the high-rise building construction as a new and innovative material compared to the normal strength concrete. In 1986, France (Malier, 1992) launched the project "New ways for concrete,” which resulted in the conception of HPC. Two breakthrough milestones are worth mentioning for the development of HPC: one is the introduction of high range waterreducing admixture (HRWRA) to concrete; the other is the advent of supplementary cementitious materials (SCM). The use of HRWRA makes it possible to produce concrete with a low water-cementitious material ratio $(\mathrm{w} / \mathrm{cm})$ while providing superior workability. The addition of SCM, as the finely divided and mostly pozzolanic and sometimes hydraulic materials, permits obtaining specific engineering properties of cement mortar and concrete. Small quantities of SCM in concrete mixtures can affect both fresh and hardened properties: fresh properties, such as setting characteristics, cohesiveness and bleeding, and heat of hydration; and hardened properties, such as strength development, ultimate strength, permeability, and durability. 


\subsection{Definition of HPC}

Different researchers have defined HPC in different ways. There have been numerous attempts to develop a definition for HPC. In 1990, the Strategic Highway Research Program (SHRP) publication (Zia, 1991) initially defined HPC, mainly focusing on the strength of the concrete. The basic requirements given by SHRP are as follows:

1) w/cm ratio not more than 0.35

2) strength at four hours not less than $21 \mathrm{MPa}(3045 \mathrm{psi})$

3) strength at 24 hours not less than $34 \mathrm{MPa}$ (4930 psi)

4) strength at 28-day not less than $69 \mathrm{MPa}(10005 \mathrm{psi})$

In 1996, SHRP further defined HPC for highway structures (Goodspeed, 1996), by identifying eight parameters as sufficient to represent long-term performance of HPC: characteristic, freeze and thaw, scalings, abrasion, chloride permeability, strength, modulus of elasticity, shrinkage, and creep. HPC were quantifiably divided into four different grades according to the requirements of those parameters; for example, HPC with shrinkage $800 \mu \varepsilon<\varepsilon_{\text {sh }} \leq 600 \mu \varepsilon$ as Grade 1, $600 \mu \varepsilon<\varepsilon_{\text {sh }} \leq 400 \mu \varepsilon$ as Grade 2, and $\varepsilon_{\text {sh }}<400 \mu \varepsilon$ as Grade 3. A given HPC mixture may be considered Grade 4 in strength and modulus of elasticity, Grade 3 in shrinkage, and Grade 2 in other categories.

In 1998, ACI finally provided an official definition for HPC (Russel, 1999), as “concrete meeting special combinations of performance and uniformity requirements that can not always be achieved routinely using conventional constituents and normal mixing, placing, and curing practices.” The special performance characteristics include:

- $\quad$ Ease of placement

- $\quad$ Compaction without segregation 
- $\quad$ Early age strength

- $\quad$ Long-term mechanical properties

- $\quad$ Low permeability

- Density

- $\quad$ Low heat of hydration

- $\quad$ Toughness

- $\quad$ Volume stability

- $\quad$ Long life in severe environment

Therefore, HPC can be defined as a special concrete, produced with appropriate materials, such as HRWRA, retarder, fly ash, blast furnace slag, silica fume and other SCM. When properly mixed, transported, placed, consolidated, and cured, HPC can attain certain superior performance characteristics like high compressive strength, high density, low permeability, and good resistance to external attack by aggressive environments.

\subsection{HPC Bridge Deck and Applications}

In the United States, most bridges were designed and constructed using concrete material. However, according to the Federal Highway Administration (FHWA)'s annual report to congress (1993), approximately $35 \%$ of the more than 570,000 bridges in the United States were classified as deficient: either structurally deficient (21\%) or functionally obsolete (14\%). The poor conditions of bridges contribute significantly to highway congestion, and safety of bridges becomes an important issue. In order to reduce the percentage of deficient bridges to a reasonable level, approximately $\$ 8.2$ billion is annually required through 2011. 
HPC, as a newly developed material provides the advantage of achieving superior durability and low maintenance. The inherent higher strength of HPC allows for greater design efficiencies. More importantly, the enhanced durability can possibly achieve longer service-life (75 years or more) of bridges. HPC may also be treated as a "green" construction material, since it incorporates recovered industrial materials which include waste byproducts such as fly ash, slag and silica fume.

As early as 1987, SHRP (Moore, 1999) was initiated to find ways to improve the constructability and reduce the maintenance of highways and bridges. In 1996, the American Association of State Highways and Transportation Officials (AASHTO) in conjunction with FHWA established the HPC Lead State Team (Moore, 1999) to promote the implementation of HPC technology in highways and highway structures, particularly, bridge deck.

As part of the AASHTO Lead State Team program, several HPC bridge projects were implemented in the participating states. Texas was the first state in the US to fully incorporate HPC into bridge practice. The first HPC bridge in Texas (Raus, 1999) was the Louetta Road overpass on State Highway 249 in Houston. The HPC mixtures were used for the deck system, precast beams, and substructure. The second HPC bridge was built in San Angelo, TX and carries the eastbound lanes of US Route 67 over the North Concho River, US 87, and the South Orient railroad. The selected HPC mixtures were used for the deck, girder and substructure. The construction of those two HPC bridges was completed in 1997. During those projects, the researchers at the University of Texas at Austin (Raus, 1999) worked closely with ready mix plants to optimize mixture designs from laboratory to the field. 
In the mid 1990s, at the beginning of their HPC program, the Virginia Department of Transportation (VDOT) already included the durability requirement in its special provision for low-permeability concrete. The first HPC bridge in Virginia (Qzyildirim, 1999) was built on Route 40 over Falling River in Campbell County. The mixture design for the deck of this bridge has a specified maximum w/cm 0.45 with an average $60.1 \mathrm{MPa}$ (8715 psi) 28-day compressive strength, and slag was used in order to achieve a better permeability property. From 1995 to 1997, a total of seven bridges were designed and constructed with HPC by VDOT.

Nebraska’s HPC bridge practice started from 1995, and a comprehensive HPC research project (Beacham, 1999) was conducted at the University of Nebraska. In total, 131 HPC mixture designs were developed and evaluated for strength, shrinkage, creep, and modulus of elasticity. The most promising mixtures were then selected and subjected to the field-level tests. The research outcome was used on Nebraska's first HPC bridge built in Sarpy County. Based on the experience gained, a special specification for HPC bridges in Nebraska was established.

In 2004, the FHWA HPC Technology Delivery Team conducted a national survey (Triandafilou, 2004) to track the HPC progress in bridge design and construction. Figure 1.1 shows the results of this survey. It was shown that a significant progress had been made since the initiation of AASHTO/SHRP Lead State Team project. To date, 34 states have included HPC in their current specifications. However, the HPC requirement for each state is found to be different, and also there seems to be long way to go to introduce consistent and comprehensive specifications in all of these states. 
In West Virginia (WV), based on the practice of several bridges including 40th Street Bridge, in Charleston, and I-64 viaduct, in Dunbar, a specification for Class H concrete was issued by the West Virginia Department of Transportation - Division of Highways (WVDOT-DOH) in July, 2003. In this specification, the w/cm for Class H concrete was 0.4 , and slag/fly ash and silica fume are required as the essential part of the concrete to achieve low permeability and better durability.

In 2002, a large-scale and comprehensive project was initiated at WVU in order to gain a better understanding of properties of HPC and accelerate its implementation in WV area. This comprehensive research project on cast-in-place HPC bridge deck has been conducted collaboration with WVDOH, contranctors, material suppliers, and ready mix concrete plants.

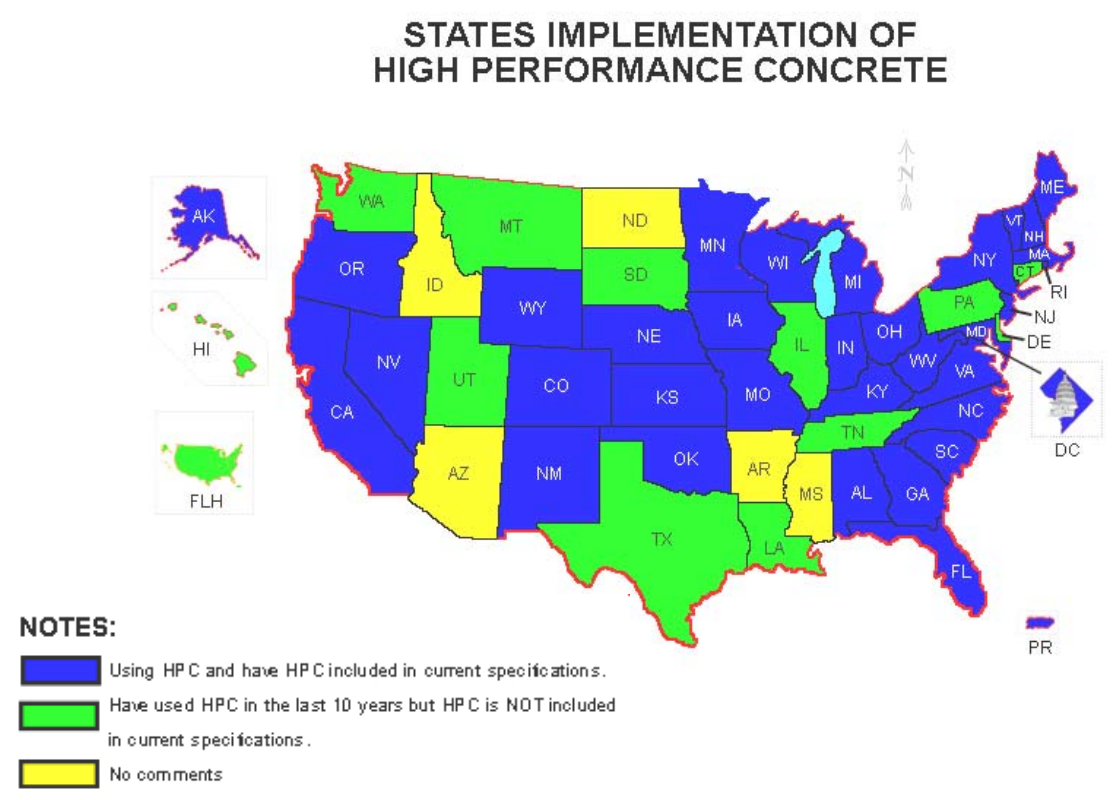

Figure 1.1 FHWA survey map (From Triandafilou, 2004) 


\subsection{Research Significance}

HPC is an engineered material and its performance is entirely dependent on the quality of local materials, local climatic conditions to which concrete will be exposed, and construction methods. Thus, it is necessary to develop suitable HPC mixtures using local material sources and construction technologies specific for a location or state.

The cracking problem has been one of the major concerns for any concrete bridge decks, especially for cast-in-place concrete decks. The cracking can accelerate deterioration of the structure, thereby increasing maintenance costs and potentially shortening the system service-life. In order to get a low permeability and reasonable strength, HPC deck mixture designs usually include a higher cementitious material content, which means more shrinkage, and therefore, makes the bridge deck more susceptible to cracking. For years, numerous studies have been focused on this topic; however, the problem still remains critical. Thus, further and better understanding of shrinkage and cracking properties of HPC designated for bridge decks is very important for its application in practices.

Over the last decades, many important discoveries concerning the causes and effects of cracking, damage, and fracture of plain and reinforced concrete structures and rock have been made by using the fracture mechanics techniques. However, only few studies have been focused on HPC, especially for use in bridge deck applications. An in-depth study of fracture mechanics properties of HPC used for bridge decks would provide an alternative and more accurate way to mitigate the current cracking problems. 


\subsection{Goals and Objectives}

The primary goal of this research is to develop low cracking or cracking-resistance HPC for bridge decks using available local materials and typical construction methods in the state of West Virginia. In this study, only the cracking cuased by shrinkage will be taken into considerations as it has been found to be the primary factor for fracture and failure of bridge decks. The proper implementation of HPC in bridge decks can increase the service-life of bridges with minimum maintenance, leading to economic benefits.

The first objective is to develop and characterize a number of HPC mixtures using representative aggregates from different locations of WV and various SCM. Fresh and mechnicical properites of all the HPC mixtures will be evaluated. The relationships between compressive strength, modulus of elasticity and direct tensile strength will be established, and the formulation for development of free shrinage tendency with time will be presented.

The second objetive is to explore cracking tendency of HPC when subjected to restrained shrinkage, by conducting ring specimen tests and monitoring crack propagations. A relationship between basic properties such as conpressvive strength and drying free shrinkage versus cracking onset days will be established based on laborarary data. This relationship can be used in the field as convenient way to evaluate the cracking properties of different practical HPC mixtures.

The final objective is to develop a numerical procedure which can be used to analyze the cracking of the ring specimens tested, as a method of validating the effectiveness of the ring specimen testing method. Also, a comprehensive fracture mechanics test will be 
conducted for selected HPC mixtures with the purpose of establishing a data set for fracture mechanics properties as a reference for future studies.

\subsection{Organization of the Dissertation}

This dissertation is organized into eight chapters as follows:

Chapter 1: A brief introduction is given about HPC and its application on bridge decks in the US, followed by the research significance, objectives and organiztion of the dissertation.

Chapter 2: Historical and theoretical backgounds of related areas are reviewed which include: shrinkage mechanisms and causes of bridge deck cracking, restrained shrinkage test methods and their evolution, and fracture mechanics of concrete related to its history and latest development.

Chapter 3: In this chapter, all the materials used in this project are listed. Their sources and detailed properties (chemical and/or physical) are included. The mixture proportions and preparation methods of the specimen are presented. A brief description of all the test methods used in this project is also included in this chapter.

Chapter 4: The test results of some basic properties of fresh and hardened concrete are discussed in the following sequence: fresh properties, compressive strength, and modulus of elasticity, direct tensile strength, and free shrinkage. Some useful relationships are estabished based on those results.

Chapter 5: This chapter is exclusively devoted to the restrained shrinkage test. The ASSHTO ring specimen test (AASHTO Designation PP34-99) is adopted. The strain development inside the steel ring, the onset of cracking in days, and crack width with 
time are presented in this chapter. A cracking index is proposed for the effective evaluation of the cracking tendency of different HPC mixtures.

Chapter 6: The R-curves of different HPC mixtures are developed based on the fracture mechanics tests. Also, G-curves of the ring specimens are developed with the aid of FEM software. A numerical prediction model about the onset of cracking for the AASHTO ring speciemen is established. The predicted data and laboratory testing data are compared.

Chapter 7: Comprehensive fracture mechanics tests are conducted on the basis of a RILEM standard procedure (RILEM 50 FCM, 1985). The fracture parameters of different HPC mixtures are compared and evaulated. Some useful relationships are estabished based on test results.

Chapter 8: Conclusions are given in this chapter, and further research needs in this area are recommended. 


\section{CHAPTER TWO}

\section{LITERATURE REVIEW}

In this chapter, historical and theoretical backgrounds of related areas are reviewed. Those include shrinkage mechanisms and cause of bridge deck cracking, restrained shrinkage test methods and their evolution, and fracture mechanics of concrete.

\subsection{Cracking Mechanism of Bridge Deck}

Cracking in concrete is always a major concern, particularly for flat structures like bridge decks. According to the survey conducted by Krauss and Rogaua (1996), more than 100,000 bridges all over the US developed early age transverse cracks. Figure 2.1 shows a typical transverse crack on a bridge deck. These cracks reduce the service life and increase maintenance costs.

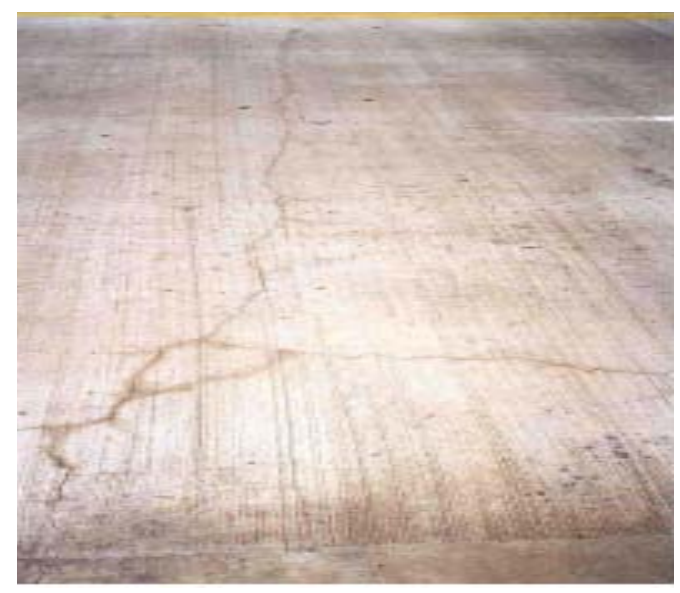

Figure 2.1 Transverse cracks on bridge deck 
The cause of cracking is a complex process, and can be the result of one or combination of several factors. Krauss and Rogaua (1996) and French et al. (1999) classified those factors into three: material, construction, and design. Figure 2.2 lists the main factors, including: shrinkage, creep, thermal stress, modulus of elasticity, curing conditions, and restraint of the structure. Among those factors, the restrained shrinkage is believed to be the key reason for causing most cracking.

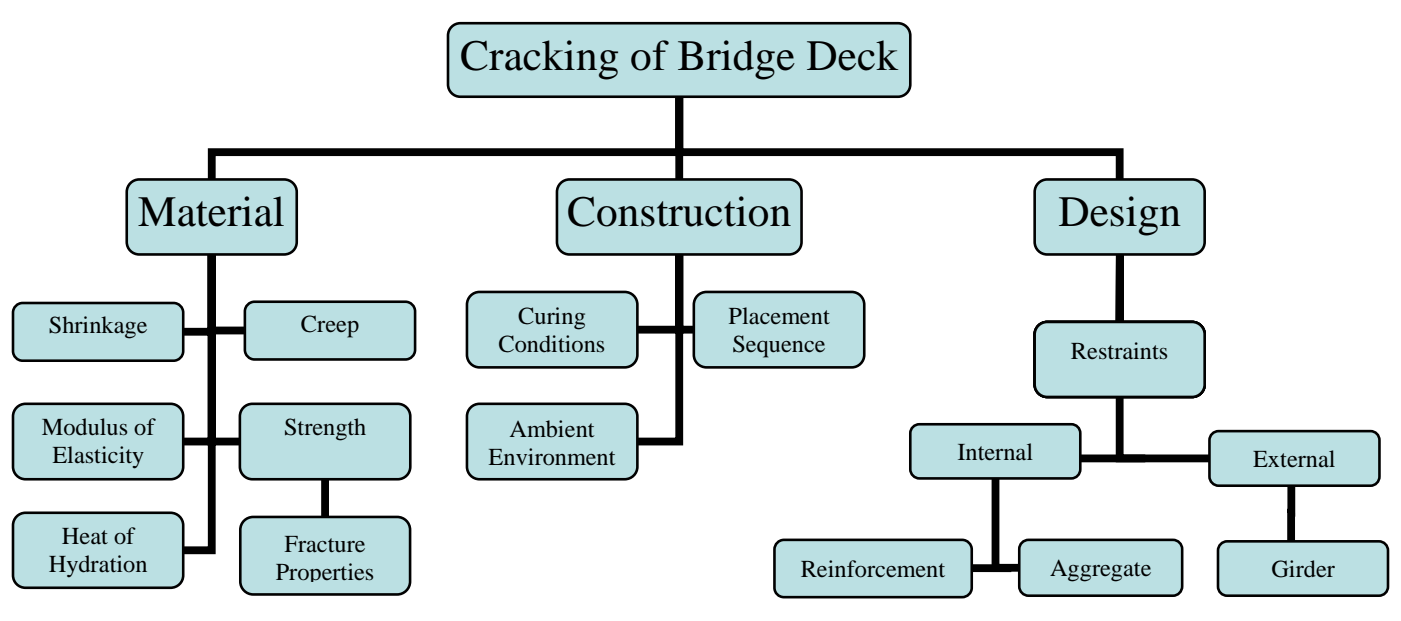

Figure 2.2 Factors related to the cracking of bridge deck

The next four sub-sections discuss: type of free shrinkage, time-dependent deformation due to creep, restrained shrinkage in bridge deck, and mechanism of cracking.

\subsubsection{Types of free shrinkage}

Concrete may undergo significant volume changes during the first hours after the initial contact of the cement and water. A brief definition of shrinkage can be the time 
dependent volumetric changes. According to different mechanisms, the shrinkage of the concrete can be further divided into the following four different types: plastic, carbonation, autogeneous, and drying.

\section{(a) Plastic shrinkage}

This kind of shrinkage occurs when the concrete is still in the plastic stage. The water in concrete mixture is lost by evaporation from the surface of the concrete or suction from dry sub-surface. When the evaporation or suction rate exceeds the rate of bleeding, and the free settlement period is ended, a hydrostatic tension begins to develop due to the formation of menisci at the water surfaces within the capillaries (Neville, 1996). As a result, concrete may be subjected to cracking unless evaporation loss is prevented.

\section{(b) Carbonation shrinkage}

When concrete is exposed to the air, the moisture inside hardened concrete reacts with carbon dioxide in the air. The carbonation forms carbonic acid in presence of moisture, which reacts with calcium hydroxide within concrete and forms calcium carbonate. The $\mathrm{pH}$ value of concrete is reduced significantly and makes the steel in the carbonated area vulnerable to corrosion. The maximum carbonation shrinkage occurs at about $50 \%$ relative humidity, and little shrinkage is observed at relative humidities close to $100 \%$ and $25 \%$. Carbonation shrinkage usually does not cause cracking per se, but it may be the reason for cracking due to lowering of $\mathrm{pH}$ and thus increasing the chance of steel corrosion.

\section{(c) Autogeneous shrinkage}

The autogenous shrinkage occurs after the setting of the cement paste has taken place and is the result of self-desiccation. As shown in Figure 2.3 (Bissonnette et al., 2001), 
when the cement particle and water are in contact, the hydration begins. During this process, if no additional water is provided (through curing), concrete will begin to dry internally and the autogeneous deformation occurs. The autogeneous shrinkage tends to increase with higher cement content, and possibly with finer cement. For HPC, due to its lower porosity and high volumes of cementitious paste, autogenous shrinkage due to selfdesiccation within concrete is significant in spite of water sprayed on the outside of the concrete decks, and sometimes, constitutes more than 50\% of total drying shrinkage.

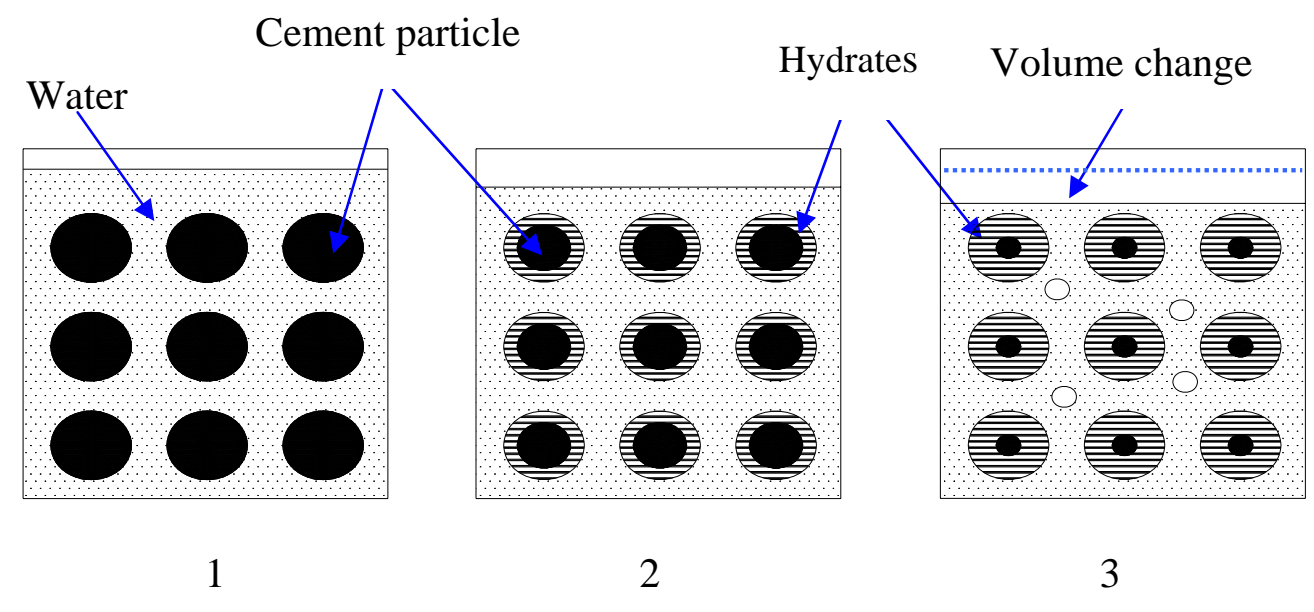

Figure 2.3 Mechanism of autogenous shrinkage

(Adopted from Bissonnette et al., 2001)

\section{(d) Drying shrinkage}

Drying shrinkage occurs in hardened concrete stored in unsaturated air when the water (free water or adsorbed water) is removed from inside of harden concrete. The lower the relative humidity of the air surrounding the concrete, the higher the rate of drying shrinkage is. Three phenomena are believed to contribute to drying shrinkage of 
concrete (Mindess et al., 2003): capillary stress, disjoining pressure, and changes in surface free energy, as shown in Figure 2.4 (a), (b), and (c) respectively.

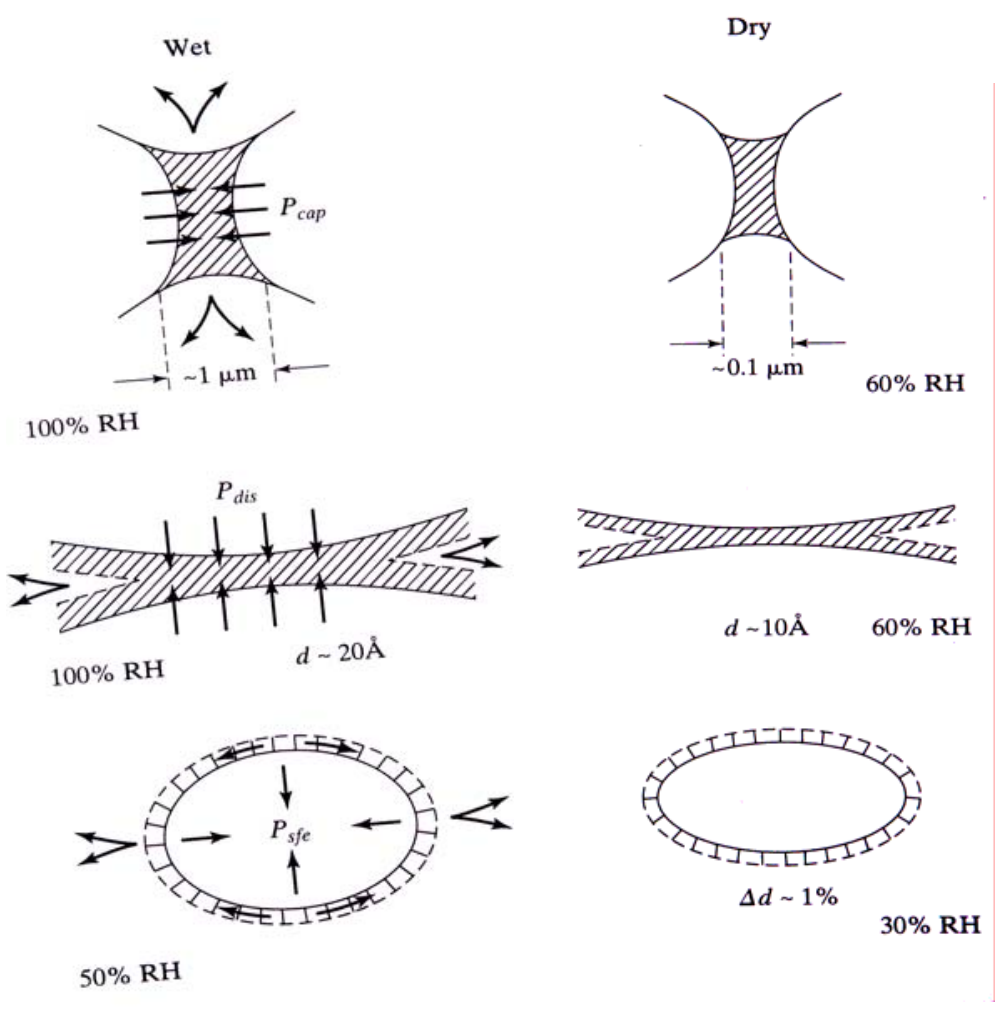

(a)

(b)

(c)

Figure 2.4 Mechanism for causes of drying shrinkage of cement paste (Reproduced from: Mindess et al., 2003)

Capillary stress: within a small capillary pore system, the water is in a state of hydrostatic tension $P_{\text {cap }}$

$$
P_{\text {cap }}=\frac{\ln (R H)}{K}
$$

where $\mathrm{RH}$ is relative humidity, and $\mathrm{K}$ is a constant.

The water exerts a corresponding hydrostatic compression on the solid skeleton. This compressive pressure is tending to reduce the volume of the cement paste. 
Disjoining pressure: There are two different forces between C-S-H (calcium silicate hydrate) gels. One is the disjoining pressure, which results from the orientation of water molecules in the absorbed film. The other is van der Waals' forces. The disjoining pressure decreases with the decreasing thickness of the absorbed water until it is lower than the van der Waals' forces, which will draw the C-S-H gels together. Disjoining pressure is only a significant factor for $\mathrm{RH}$ down to about $45 \%$, and it shows an $\mathrm{RH}$ dependency similar to capillary stress.

Surface free energy: when the RH is between $20 \%-45 \%$, the drying shrinkage can be explained by changes in surface energy. The changes of the surface energy results in a hydrostatic pressure as expressed in the following equation:

$$
p_{s f e}=\frac{2 \Delta \gamma S}{3}
$$

where $\gamma$ is the surface energy, $S$ is the specific surface area of a solid. The solid C-H-S gel is likewise subjected to a mean pressure, resulting in shrinkage.

Autogeneous shrinkage is a special case of drying shrinkage in which drying takes place internally due to self-desiccation. As far as HPC is concerned, the autogenous shrinkage plays a more important role than drying shrinkage due to higher volume of cementitious materials, lower $\mathrm{w} / \mathrm{cm}$ and formation of more micropores. The free shrinkage measured in laboratory as length change from very early age (1-day) consists of both autogeneous and drying shrinkage, which means the volume change is due to both internal and external drying.

\subsubsection{Creep}

When concrete is subjected to a stress, a time-dependent deformation begins immediately and continues for as long as the concrete is loaded. This time dependent 
deformation is called creep. Excluding the influence of applied load, the same as for shrinkage, the factors influencing creep of concrete include w/cm, curing conditions, temperature, humidity, cementitious materials, aggregates and specimen geometry. There are two types of mechanisms: basic creep, which occurs in the absence of hygrometric exchanges with the ambient medium, and drying creep, that appears when the loaded concrete dries. The total strain in a concrete specimen includes the effect of: initial elastic strain due to applied load, shrinkage strain, thermal strain, and creep strain overtime. Further details of creep phenomena may be found in several literatures (Neville, 1996 and Mindess et al., 2003)

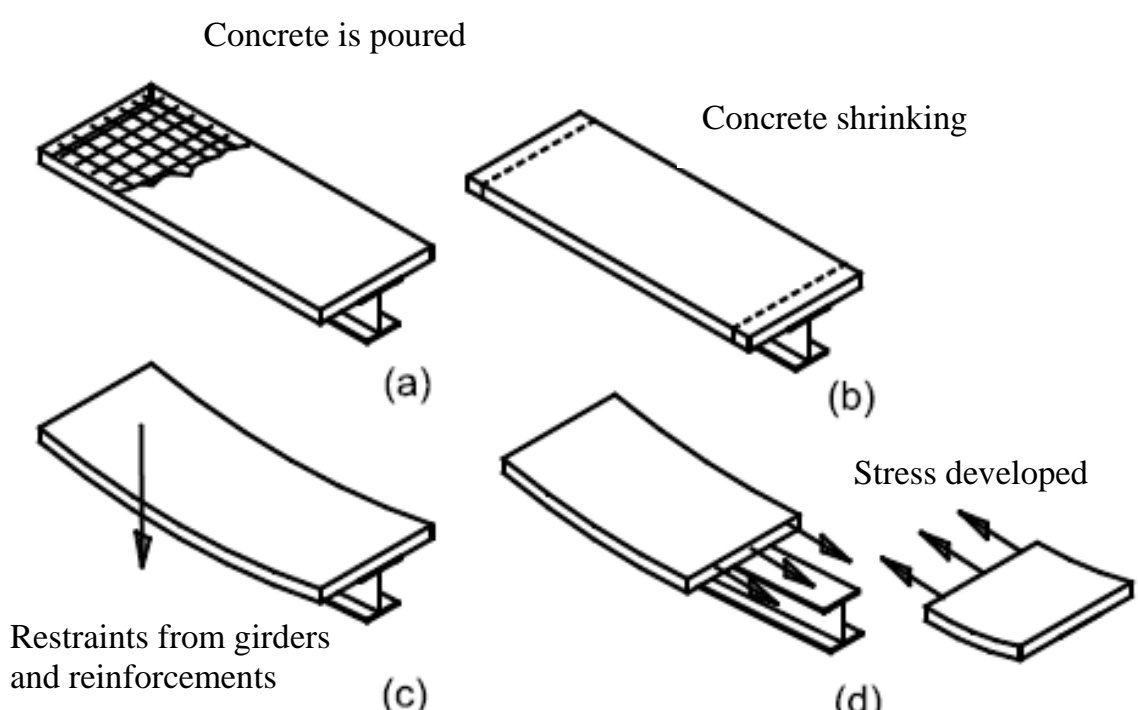

Figure 2.5 Mechanism of restrained shrinkage of bridge deck

(Reproduced from: Saadeghvaziri and Hadidi, 2002) 


\subsubsection{Restrained shrinkage in bridge decks}

The free shrinkage itself does not induce any stress, however if the deformation is restrained, the force and pressure provided by the restraint can cause stress. The restraints can be internal such as aggregate and reinforcement or external such as girder or sub-base (Figure 2.2). A concrete bridge deck is particularly susceptible to both internal and external restraints. As shown in Figure 2.5 (Saadeghvaziri and Hadidi, 2002), most bridge decks are designed as composite structure with supporting steel girders. The composite action of deck and girder, as well as the stiffness difference between concrete and steel, increase the restraints.

\subsubsection{Mechanism of cracking}

Under restraining conditions present in concrete structures, such as bridge decks, as shrinkage effect develops, the tensile stress is induced and accumulated inside the concrete, and as a result, cracking may occur. However this is a complicated process and is the result of the interaction among tensile stresses induced by shrinkage, modulus of elasticity, and stress relief due to creep relaxation. For a given concrete, time has a twofold effect (Neville, 1996): the tensile strength will increase with time, but at the same time the modulus of elasticity will increase as well, so that the induced stress by a given amount of shrinkage becomes larger. As shown in Figure 2.6, the cracking can be avoided only if the relaxation tensile stress is lower than the concrete tensile strength. 


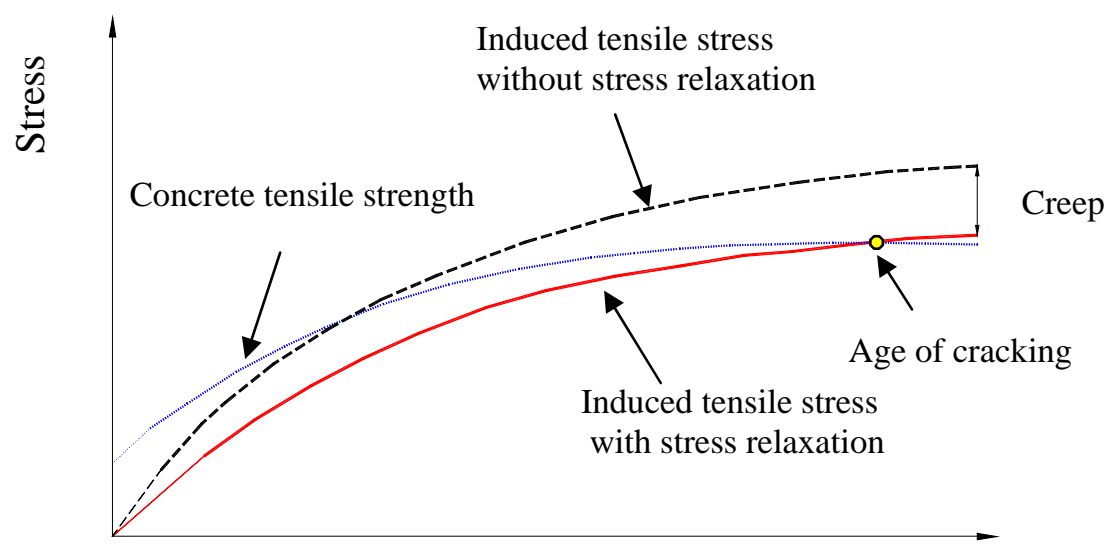

Time

Figure 2.6 Mechanism of cracking (From Neville, 1996)

For HPC, due to the higher self-desiccation as mentioned in Section 2.1.1, the early age free shrinkage is much higher compared to that in normal concrete, while at the same time the tensile strength and creep relaxation are still low. Therefore HPC is more susceptible to early cracking unless adequate measures are taken to reduce early age shrinkage.

\subsection{A Review of Restrained Shrinkage Test Methods}

In order to evaluate the cracking tendency of different concrete mixtures, a number of retrained shrinkage test methods have been developed. These tests are conducted on three different types of specimens: bar, plate, and ring, as described below.

\subsubsection{Bar test}

The bar specimen test is also called uniaxial restrained shrinkage test, which provides relatively uniform uniaxial state of stress along the specimen tested. There are two types of uniaxial restrained specimens. One is so-called "dog-bone” specimen, and the other one is prismatic beam specimen. 
Figure 2.7 shows the so-called cracking frame, which was first developed in Germany (Springenschmid et al., 1994). The “dog-bone” type specimen is cast horizontally and has a straight prismatic central section. This device includes two steel bars running longitudinally and two steel cross heads at each end. The frame was initially designed for thermal cracking study.

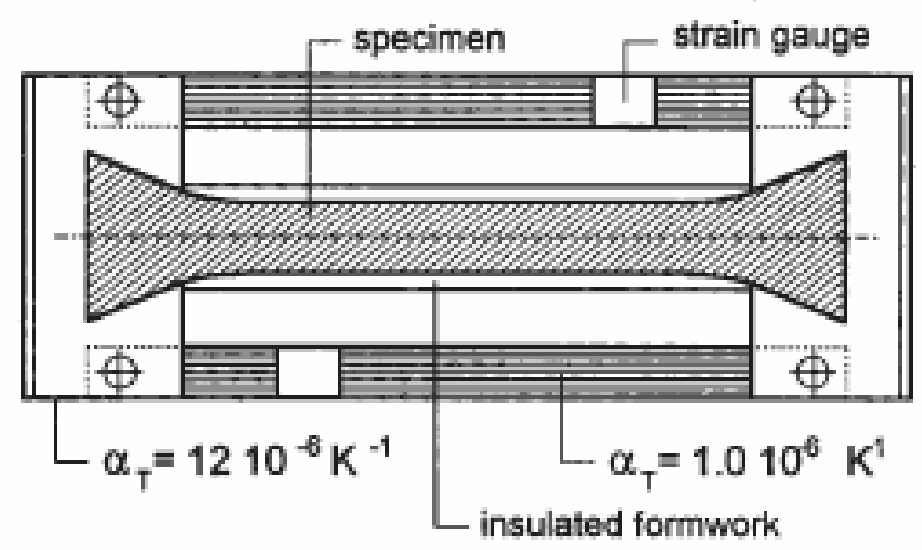

Figure 2.7 The cracking frame apparatus (Reproduced from: Springenschmid et al., 1994)

Kolver (1994); Bloom and Bentur (1995); and Igarashi et al. (2000), conducted several restrained shrinkage and tensile creep tests using modified cracking frame devices. Figure 2.8 shows the restrained shrinkage testing system used by Igarashi et al. (2000). Two concrete specimens were used, one free and the other restrained. For the restrained specimen, one grip was fixed, while the other one was free to move, and a LVDT (or special gauge) was used to monitor the movement of the free grip. The computer controlled motor was programmed to pull back the free grip to its original position. The 
whole system can achieve a fully automated closed loop control as well as high accuracy of measurements.

The other type of bar test is conducted on a prismatic beam specimen, in which threaded rods are embedded to provide restraint. The prism specimen test was adopted by ASTM C 878, including an assembled restraining cage to measure the length change of shrinkage compensating concrete under restrained condition.

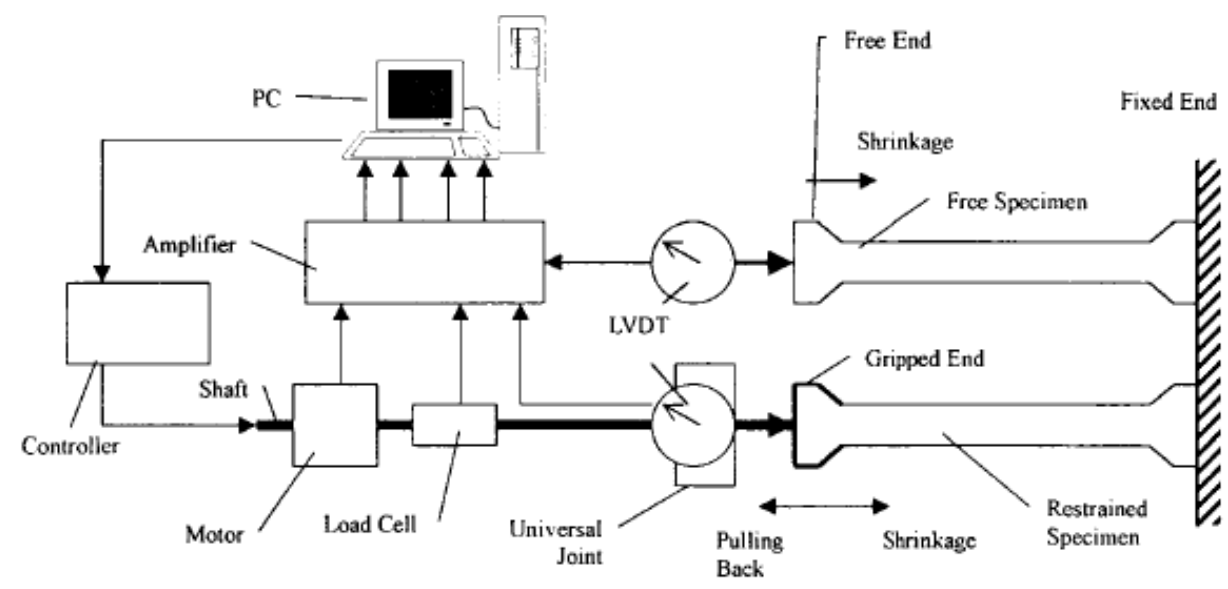

Figure 2.8 Scheme of restrained shrinkage testing system

(Reproduced from: Igarashi et al., 2000)

The Japan Concrete Institute (JCI) developed a standard test device (JCT, 1996) to measure the shrinkage properties of concrete. The test setup is shown in Figure 2.9. Similar to the ASTM C 878, a deformed steel rebar is embedded with a smooth central portion covered with Teflon sheet. A sufficient length of smoothed rebar is used to produce a uniform stress distribution in both materials. 


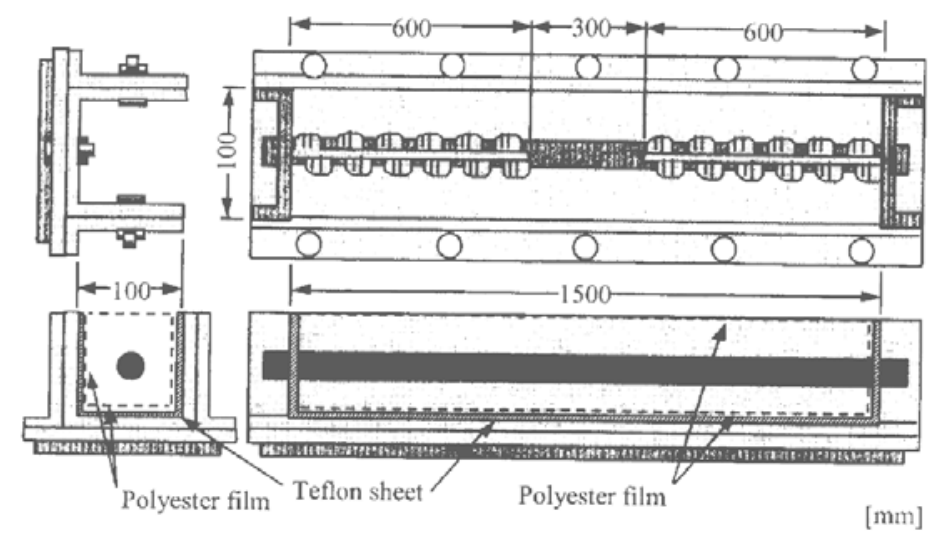

Figure 2.9 JCI restrained shrinkage test device

(Reproduced from: JCI, 1996)

For the bar type specimen test, it is difficult to provide sufficient restraint to produce cracking with linear specimens, especially when the cross-sectional dimensions are large. The method by Igarashi et al. (2000) provides an innovative way to maintain the restraint. However, the whole test configuration is complicated, and difficult to implement for large number of tests.

\subsubsection{Plate (slab) Test}

The plate or slab specimen is usually used for the evaluation of plastic shrinkage cracking. However the plate or slab specimen is a good choice to simulate the restraint experienced in an actual structure such as a bridge deck, or an industrial floor.

Kraai (1985) employed a plate specimen to study the effect of material composition on plastic shrinkage cracking. The dimension of the specimen was $0.9 \mathrm{~m} \times 0.6 \mathrm{~m} \times 0.02 \mathrm{~m}$ (35.4 in $\times 23.6$ in $\times 0.8$ in). Since a plate type specimen is similar to the actual structural system, the information obtained from this method can be directly applied to a specific structure. The main limitation of this method is that the degree of restraint is much less 
than $100 \%$. This is further complicated by the constant change in level of restraint with time because of both continual change in hydration and viscoelastic nature of concrete.

Weiss et al. (1998) developed slab type specimens to study the restrained cracking of concrete with shrinkage reducing admixtures. One type of slab was restrained at the ends. The other was restrained both at the ends and at the bottom base. The cracking onset days were recorded and compared.

Bathia et al. (1996) developed a novel method to study a concrete overlay which is subjected to sub-base restraint as shown in Figure 2.10. The whole test was conducted in an environmentally controlled chamber. The concrete base was cast first and roughened manually to increase the restraining force. The upper overlay was then cast over the substrate. The cracking onset days were monitored.

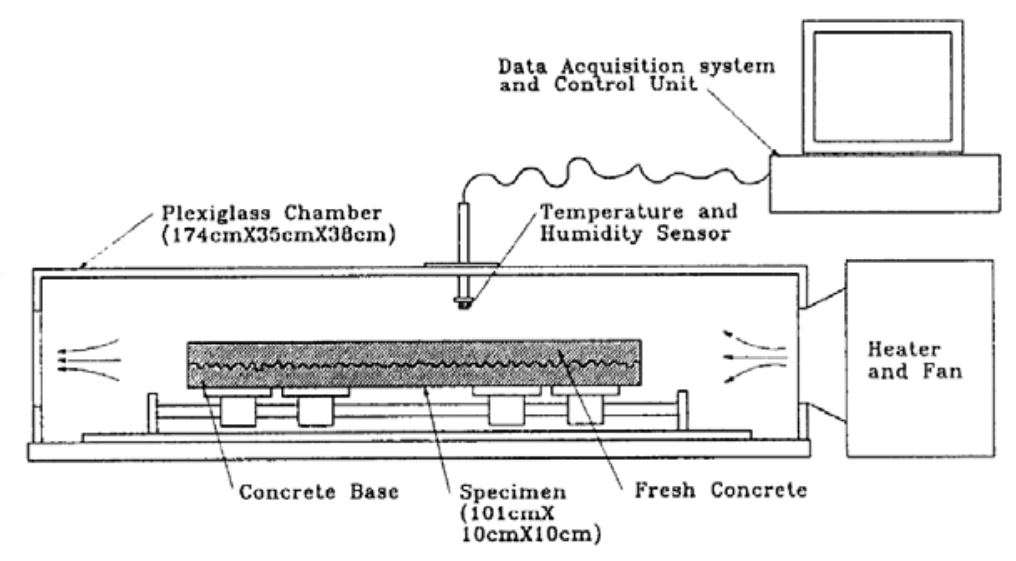

Figure 2.10 Slab test scheme

(Reproduced from: Banthia et al., 1996) 


\subsubsection{Ring specimen test}

The ring test was first developed by Carlson in early 1940s. His work was first published in 1942 (Carlson, 1942), then subsequently published in 1988 (Carlson and Reading, 1998). In this test, a 25.4 mm (1 in) thick steel ring with an external diameter of $178 \mathrm{~mm}$ (7 in) was used as a restraint source. The concrete or mortar ring specimens were cast around the exterior surface of steel rings. As a result of drying, the concrete ring shrinks, and exerts axisymmetric pressure over the "rigid" steel rings, leading to tension cracking on the outer surface of the concrete ring specimen. The cracking onset depends on the degree of drying of concrete, stiffness of inner steel, and concrete material properties.

Bennet and Loat (1970) employed a ring test method based on the work by Coutinho (1959) to investigate the shrinkage properties of Portland cement with different fineness. In their tests, three silicon semiconductor strain gauges, which gave a better sensitivity than the vibrating wire, were mounted to sense the deformation of the steel ring. Almost at the same time, Malhotra (1970) in Canada used the ring test method to determine the tensile strength of concrete. The outside diameter of steel ring used was 304.8 mm (12 in). The dimensions of the concrete ring were $76.2 \mathrm{~mm}$ in thickness $\times 76.2 \mathrm{~mm}$ in height (3 in $\times 3$ in). The relationship between the tensile strength from ring test versus cylinder compressive strength was established.

From the late 1980s to1990s, a number of studies were conducted in the US with ring type specimens. Grzybowski and Shah (1990) investigated the shrinkage cracking of fiber reinforced concrete with a ring specimen. In their test (Figure 2.11), holographic technology and a special microscope setup were first applied to detect the presence of 
microcracks on the surface of ring specimens and to measure crack widths. In 1995, the same method (Wiegrink et al., 1996) was applied to investigate the shrinkage cracking properties of high strength concrete. In 1998, a numeric method (Shah et al., 1998) based on fracture mechanics, was established to predict shrinkage cracking of the ring type concrete specimen.

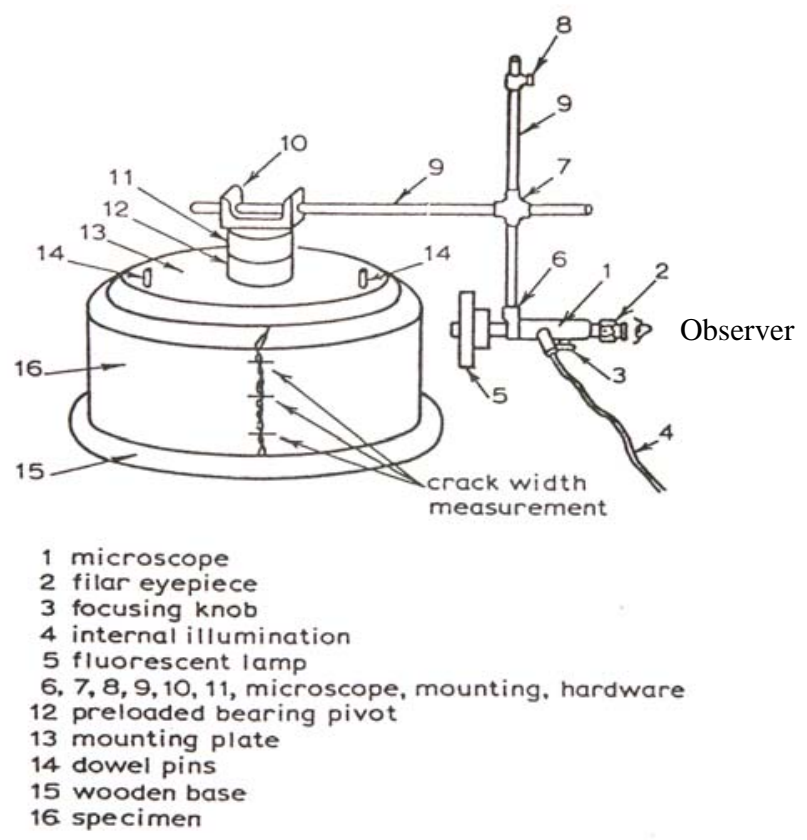

Figure 2.11 Ring specimen test setup

(Reproduced from: Grzyboski and Shah, 1990)

In 1998, AASHTO approved a provisional standard for the ring test, as "Standard Practice for Estimating the Cracking Tendency of Concrete." The steel ring recommended has a wall thickness of $12.8 \mathrm{~mm}\left(\frac{1}{2} \mathrm{in}\right)$, an outside diameter of $305 \mathrm{~mm}$ (12 in), and height of $152 \mathrm{~mm}$ (6 in). Four strain gages are suggested to be mounted to the interior surface of the steel ring on opposite directions to monitor the onset of concrete cracking. In practice, a smaller size of ring is also sometimes used if aggregate for concrete mixture is small. 


\subsection{Fracture Mechanics of Concrete}

In this section, the historical background on the development of fracture mechanics is reviewed, and followed by the concept about the fracture process zone around crack tip inside concrete. Several fracture mechanics models for concrete are also presented.

\subsubsection{History of fracture mechanics from Griffith approach to cohesive crack model}

The original concept of fracture mechanics started on concepts of linear elastic fracture mechanics (LEFM), which can be traced back to the 1920s by Griffith (1920). Griffith's theory begins from the hypothesis that brittle material contains microcracks which can introduce high stress concentrations near their tips. Based on his test results, he first proposed the concept of Griffith energy criterion of fracture for brittle materials, which can be expressed as:

$$
\frac{\partial(W+\Gamma)}{\partial a}=0
$$

Where $W=-\frac{\pi a^{2} \sigma^{2}}{E}$ is the work done; $\Gamma=4 a \gamma$ is the surface energy; $a$ is crack extension length, and $\gamma$ is unit surface energy. According to Griffith energy criterion, as the crack grows by a small amount $\partial a$, to get stable crack propagation, the above energy balance condition (Eq. 2.3) should be satisfied.

In 1957, Irwin suggested a new modification (Irwin, 1957) of the Griffith theory of brittle fracture to generalize Griffith's theory to the case of quasi-brittle materials. For information purpose, Irwin's main contributions to the development of fracture mechanics are summarized next from the writing by Anderson (1995): 
1) He introduced the concept of effective surface energy and reformulated the Griffith theory in terms of singular stresses at the crack front and proved that his approach was equivalent to the Griffith’s energy approach.

2) He introduced stress intensity factors $\mathrm{K}$ : a parameter which relates the local mechanical state near the crack tip to macroscopic characteristics, and showed that the stress field in the area of crack tip is completely determined by the quantity $\mathrm{K}$, which enables one to give a very simple description of fracture processes as a continuous process of stable crack growth up to the onset of instability.

3) Irwin initiated investigation of various aspects of the theory of fracture such as the role of plasticity, dynamics of the process, and fractographic analysis of crack growth, etc. His ideas were very important for practical use and subsequent works, and caused a decisive influence on the progress in fracture mechanics as a new science and powerful engineering tool applicable to numerous problems connected with manufacturing and processing of materials and their failure proof operation in critical structures of various materials.

Soon after the introduction of fracture mechanics concept, it became evident that LEFM has good predictions only when the material is very brittle. However this is not the case for most practical structures. During the 1960s, various nonlinear fracture mechanics theories were developed. Among those theories, the first attempt, which included the cohesive forces in the crack tip region within the limits of elasticity theory, was made by Barenblatt (1959). In Barenblatt's theory, as shown in Figure 2.12(a), it was assumed that a cohesive stress $\sigma(x)$, for which the crack faces can close smoothly, acted in a small zone (cohesive zone) near the crack tip. However, the distribution of such cohesive 
stresses is generally unknown. Then Dugdale (1960) added some modifications to Barenblatt's theory and developed his own cohesive model. As shown in Figure 2.12 (b), Dugdale's model is almost the same as that of Barenblatt's cohesive crack model, except that the distribution of the cohesive stresses is known and constant, and therefore there is no need to require the invariance and smallness of the cohesive zone.

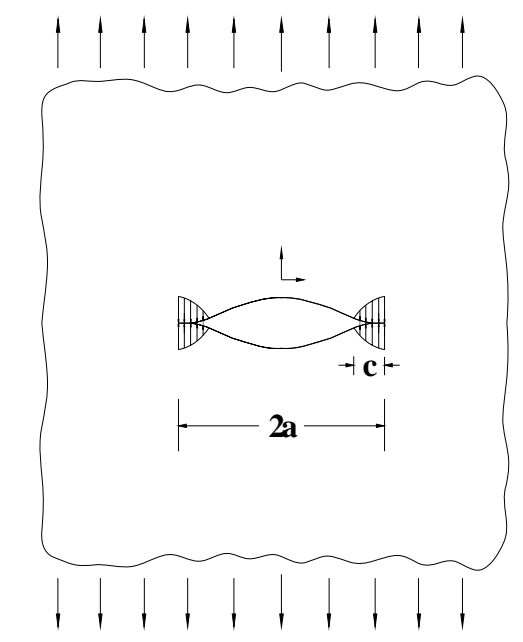

(a) Barenblatt's cohesive model

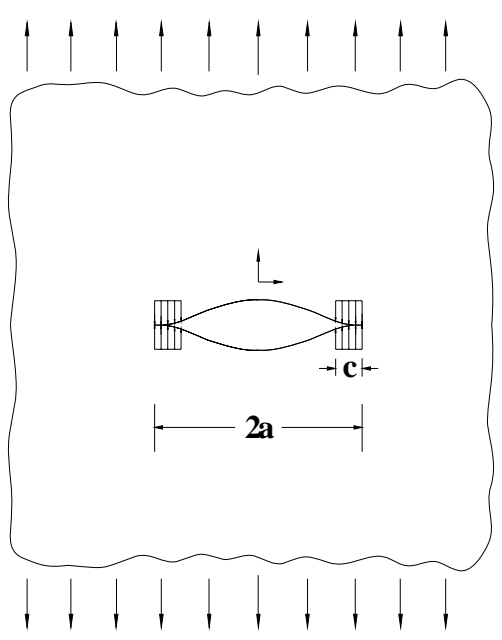

(b) Dugdale's cohesive model

Figure 2.12 Barenblatt and Dugdale’s cohesive models

Rice (1968) suggested a new parameter (J-integral) for fracture of metals. This parameter is independent of the integration path around the crack tip and is also used as a crack growth criterion.

\subsubsection{Fracture process zone (FPZ) of concrete}

LFEM allows the stress to reach infinity at the crack tip. However, in real materials, a certain range of inelastic zone must exist at the crack tips. For the ductile materials, such as metal, the inelastic zone is a nonlinear plastic zone. Whereas, in quasi-brittle materials 
like concrete, an inelastic zone around the crack tip is known as so-called fracture process zone. Many mechanisms are responsible for fracture process inside the concrete, and some main toughening mechanisms are discussed in this section.

As it is known, concrete is a heterogeneous material consisting of different phases. Flaws, such as water filled pores, air voids, bleed water lenses under coarse aggregates and shrinkage cracks, exist even before loading. Due to the stress concentration ahead of a flow, microcracks could be initiated at nearby flaws and propagate as shown in Figure 2.13 (a). This is also known as the microcrack shielding, which can consume a part of external energy.

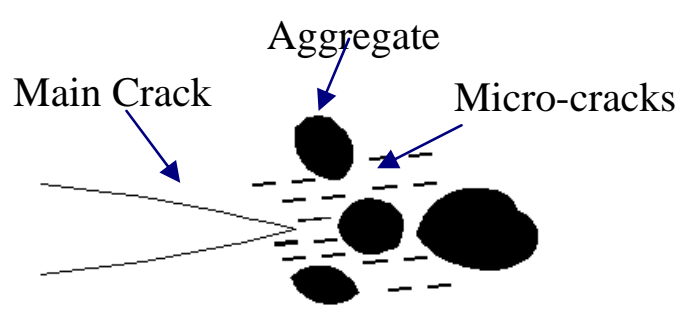

(a)

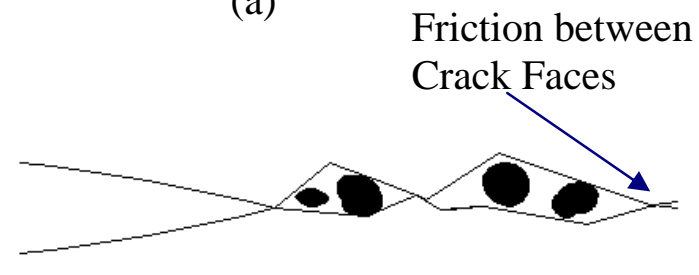

(c)

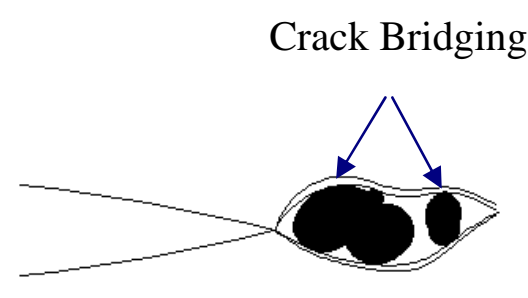

(b)

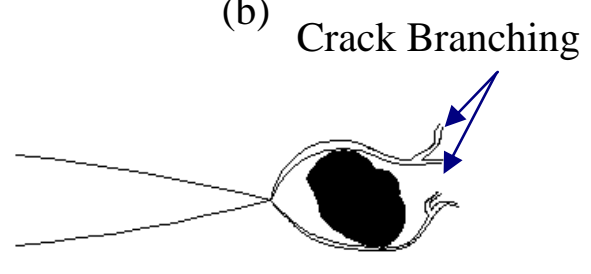

(d)

Figure 2.13 Fracture toughening mechanisms

The crack may be forced to grow around the strong aggregates, as a result, the crack deflection occurs. When the crack has advanced beyond the aggregate, an aggregate bridge will be formed to transmit the stress between the faces of crack. This process is 
called crack bridging, one very important fracture toughening process, as shown in Figure 2.13 (b).

As the crack propagates, there may be some contact between the cracked faces, due to the friction and bridging across the crack, and as a result, part of the energy will be dissipated, as shown in Figure 2.13 (c). Since concrete is a heterogeneous material, and the properties in different directions may not be same, the crack may propagate into different branches, as shown in Figure 2.13 (d). This kind of toughening mechanism is called crack branching.

\subsubsection{Fracture theories for concrete}

In this section, the concept and development of fracture mechanics models for concrete are discussed. Those models include nonlinear cohesive models and approximate nonlinear models.

\subsubsection{Introduction}

Due to existence of the sizable fracture process zone at the tip of the crack, LEFM can not be directly applied to concrete for normal size specimens. Figure 2.14 shows a typical stress-strain curve for a concrete in tension. It is observed that the pre-peak nonlinearity and the post-peak tension-softening are primarily the result of microcracking, aggregate bridging, and other toughening mechanisms. In order to account for the fracture process zone into the fracture theory for concrete, several nonlinear approaches have been made during the last two decades. Those attempts can be divided into two categories: nonlinear cohesive models, and approximate nonlinear models. 


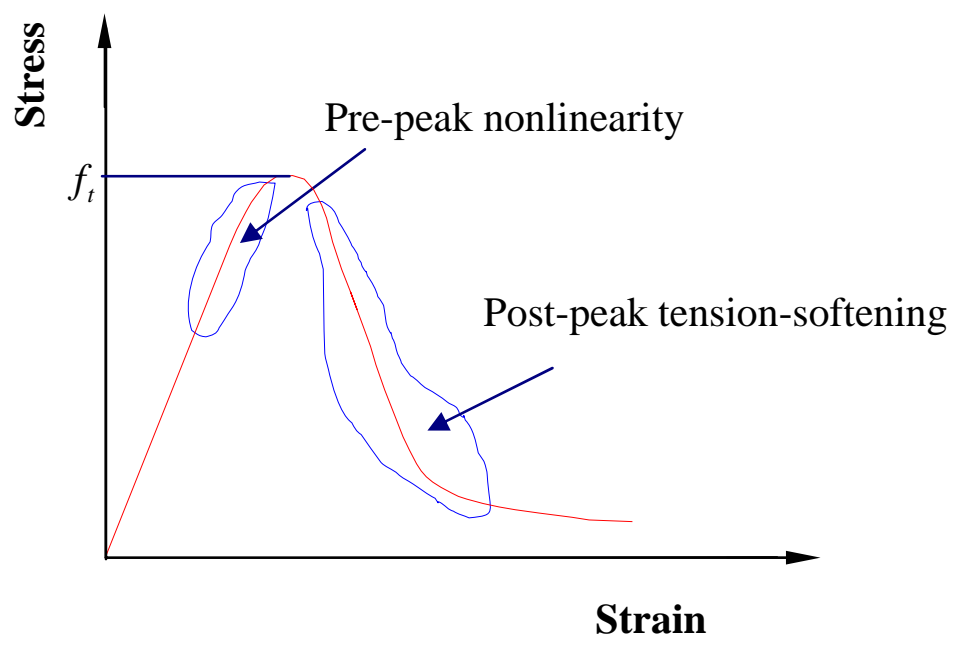

Figure 2.14 Typical stress-strain curve for concrete in tension

\subsubsection{Nonlinear cohesive models}

Two nonlinear cohesive models: fictitious crack and crack band model are discussed in this section.

\section{(a) Fictitious crack model (FCM)}

As mentioned before, the cohesive model was introduced in the early 1960s by Barenblatt (1959) and Dugdale (1960). Hillerborg et al. (1976) first extended the concept of cohesive crack for fracture of concrete. Hillerborg's theory includes a tension softening fracture process zone through a fictitious crack ahead of the pre-existing crack, as shown in Figure 2.15 (a). It is different from Dugdale's theory as the closing stress in the fracture process zone is not constant. The stress distribution increases from zero at the tip to the full tensile strength value of the material $f_{t}$. The distribution of the closing stress along the process zone depends on the crack opening displacement $w$. 
Based on the uniaxial tension test on a concrete bar, Hillerborg described the tensile stress-elongation curve in an idealized manner. The prepeak tensile response of concrete can be described by a stress-strain curve as shown in Figure 2.15 (b), whereas the postpeak fracture behavior can be characterized as stress-elongation curve as shown in Figure 2.15 (c). The area under the entire softening stress-elongation curve is expressed as:

$$
G_{F}=\int_{0}^{w_{c}} f(w) d w
$$

where $G_{F}$ is fracture toughness; $w_{c}$ is the critical tip opening displacement at the time when the softening stress $f(w)$ is equal to zero. For a given material, the fracture property is determined by the tensile stress $f_{t}$, the fracture toughness $G_{F}$, and the shape of the softening curve. If the shape of $f(w)$ is given, the fracture is only determined by $G_{F}$ and $f_{t}$.

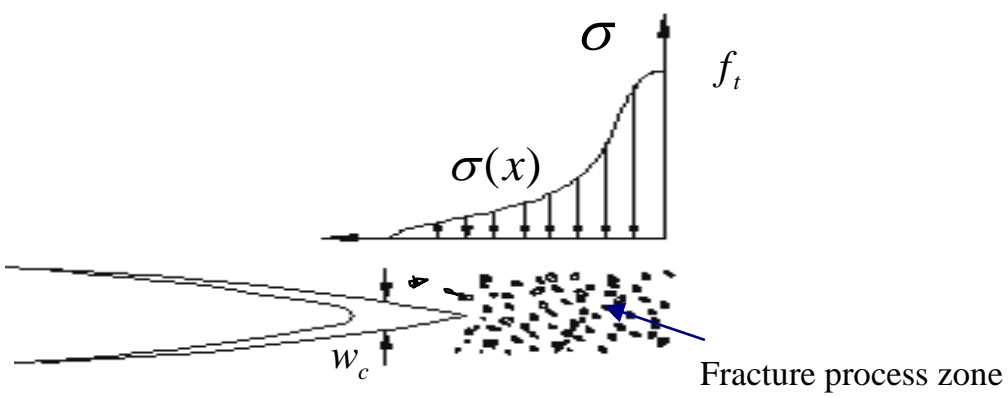

(a)

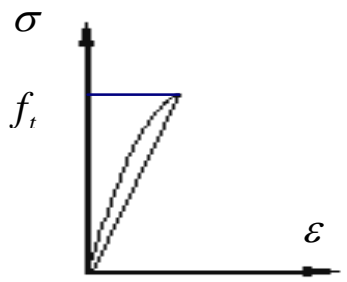

(b)

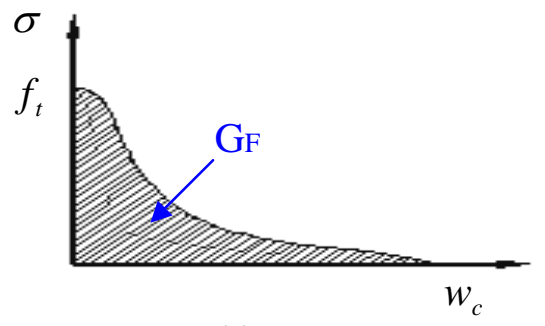

(c)

Figure 2.15 Hillerborg's fictitious theory (Adopted from: Hillerborg et al., 1976) 
Hillerborg also proposed the concept of the characteristic length $l_{c h}$, which is expressed as:

$$
l_{c h}=\frac{E G_{F}}{f_{t}^{2}}
$$

where $E$ is the modulus of elasticity; $G_{F}$ is fracture energy, and $f_{t}$ is tensile strength.

The characteristic length combines two parameters: $G_{F}$ and $f_{t}$ together, and is considered as a purely material property.

\section{(b) Crack band model (CBM)}

Bazant and Oh (1983) assumed that the microcracks in the fracture process zone are distributed over a band of width $h$, as shown in Figure 2.16 (a). The deformation was smeared over this band. This model is also called smear crack approach. The tensionsoftening relation can be described by a simple stress-strain curve, which is shown in Figure 2.16 (b). The fracture toughness can then be expressed as:

$$
G_{f}=h\left(1+\frac{E}{E_{t}}\right) \frac{f_{t}^{2}}{2 E}
$$

where $E$ is the modulus of elasticity; $E_{t}$ is the strain -softening modulus; $f_{t}$ is the tensile strength, and $h$ is the width of the crack band which is related to $w_{c}$ (critical crack tip opening displacement) and $\varepsilon_{c}$ (critical strain). On the basis of test data, Bazant recommended a value of $h \approx 3 g$ ( $g$ is the maximum aggregate size) 


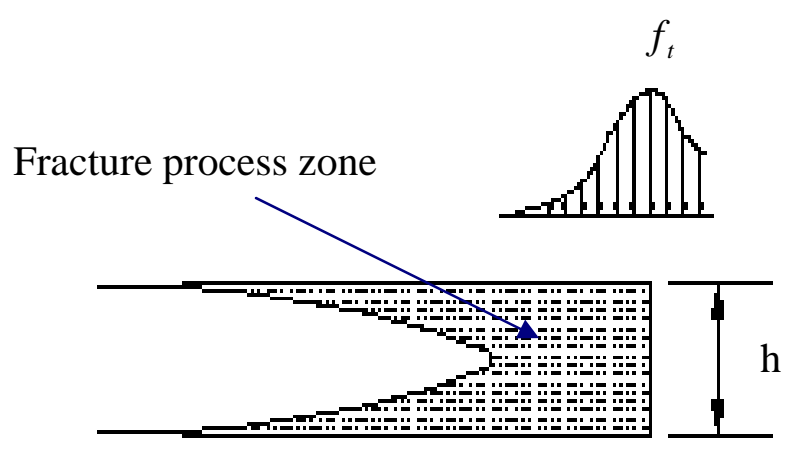

(a)

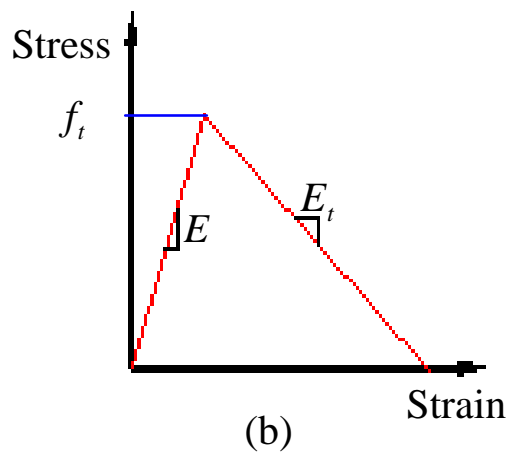

(b)

Figure 2.16 Crack bond theory

(Adopted from: Bazant and Oh, 1983)

\subsubsection{Approximate nonlinear models}

The cohesive model assumes a cohesive stress ahead of crack tips which follows the stress-displacement or stress-strain softening law. However, in practice, the abovementioned cohesive model requires a sophisticated analysis method for its implementation. Several models have been proposed as the adaptations of LEFM by taking into account the nonlinear fracture behaviors (fracture process zone) in an approximate way. Those approaches include: two-parameter, size effect, and effective crack models.

\section{(a) Two-parameter model ( TPM)}

Jenq and Shah (1985) first proposed the two parameter fracture model, which includes the effect of pre-peak nonlinear behavior through a critical effective crack length $a_{c}$. Figure 2.17 shows a typical loading and unloading curve for a three-point bending test. The specimen may be loaded up to the maximum stress, and then unloaded. The value of $C_{u}$ is the unloading compliance. The inelastic component of the $C M O D^{P}$ is reduced from the total $C M O D$. The critical elastic $C M O D_{c}{ }^{e}$ as well as critical stress $\sigma_{c}$ 
are used to calculate the critical stress intensity factor $K_{I C}{ }^{s}$ and critical crack-tip opening displacement $C T O D_{c}$ following the LEFM equations.

Based on experimental results, Jenq and Shah (1985) found that the values of $K_{I C}{ }^{s}$ and $C T O D_{c}$ are basically constant for the same material independent of the sizes. Those two parameters are considered as the material properties of quasi-brittle materials like concrete. Therefore, when structures with the same material but different size and geometries are subjected to critical fracture loads, the following two conditions will be satisfied:

$$
K_{I}=K_{\text {Ic }}^{s} \quad C T O D=C T O D_{c}
$$
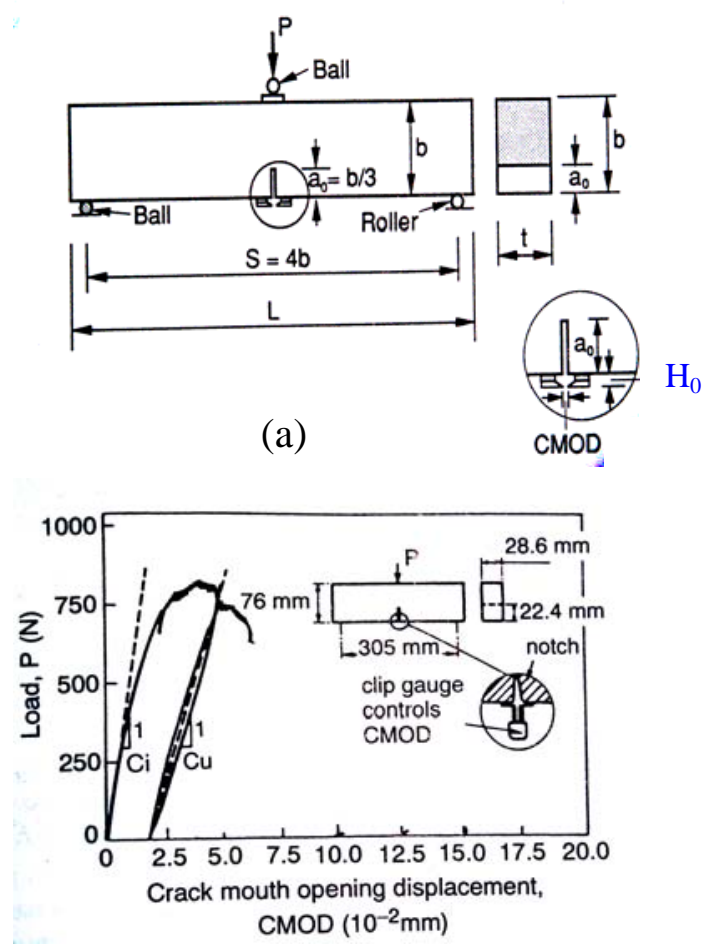

(b)

Figure 2.17 Two parameter model

(Reproduced from: Jenq and Shah, 1985) 


\section{(b) Size effect model (SEM)}

The idea of size effect model comes from the concept that LEFM can be applied to the analysis of concrete structures with infinitely large dimensions of any shapes. Based on the assumption that the fracture energy dissipated at failure is a smooth function of structural dimensions and size of fracture process zone, Bazant and Kazemi (1990) proposed the following scale law for a series of geometrically similar structures with different sizes:

$$
\sigma_{N c}=B_{0} f_{t}\left[\frac{d}{d_{0}}+1+L_{1}\left(\frac{d}{d_{0}}\right)^{-1}+L_{2}\left(\frac{d}{d_{0}}\right)^{-2}+\cdots\right]^{-1 / 2}
$$

where $\sigma_{N c}$ is nominal stress at the maximum load; $B_{0}$ and $d_{0}$ are two material constants; $f_{t}$ is tensile stress; $d$ is the depth of the concrete structure. Eq. (2.8) can be further simplified as:

$$
\sigma_{N c}=\frac{B_{0} f_{t}}{\sqrt{1+\frac{d}{d_{0}}}}
$$

Based on the formulation from LEFM, Bazant and Kazemi (1990) proposed two parameters for Mode I fracture for concrete, as

$$
\begin{gathered}
G_{f}=\lim _{d \rightarrow \infty} \frac{\sigma_{N c}{ }^{2} \pi\left(a_{0}+\Delta a_{c}\right)}{E} g_{1}{ }^{2} \\
c_{f}=\lim _{d \rightarrow \infty} \Delta a_{c}
\end{gathered}
$$

where $G_{f}$ and $c_{f}$ are the critical energy release rate and critical crack extension for infinitely large structures, respectively; $\Delta a_{c}$ is effective crack extension, and $g_{1}$ is geometric function. 


\section{(c) Effective crack model (ECM)}

Nallathambi and Karihaloo (1986 and 1989) first proposed a method to calculate the effective crack length by the concept of the secant stiffness of the real beam at the peak load. As shown in Figure 2.18, for a three-point bending of a beam with an initial crack length $a_{0}$, the stiffness in the linear regime can be calculated at any point with an equation based on the finite element method:

$$
\begin{aligned}
E & =\frac{P_{i}}{4 t \delta_{i}}\left(\frac{S}{b}\right)^{3}\left[1+\frac{5 S W}{8 P_{i}}+\left(\frac{b}{S}\right)^{2}\left(2.7+1.35 \frac{S W}{P_{i}}\right)-0.84\left(\frac{b}{S}\right)^{3}\right] \\
& +\frac{9 P_{i}}{2 t \delta_{i}}\left(1+\frac{S W}{2 P_{i}}\right)\left(\frac{S}{b}\right)^{2} g_{4}\left(\frac{a_{0}}{b}\right)
\end{aligned}
$$

where $P_{i}$ and $\delta_{i}$ are the load and corresponding deflection; $\mathrm{E}$ is the stiffness of the beam; $S, b$ and $t$ are the span, depth and thickness of the beam, respectively; $W$ is the self-weight of the beam; $g_{4}$ is a geometric function;

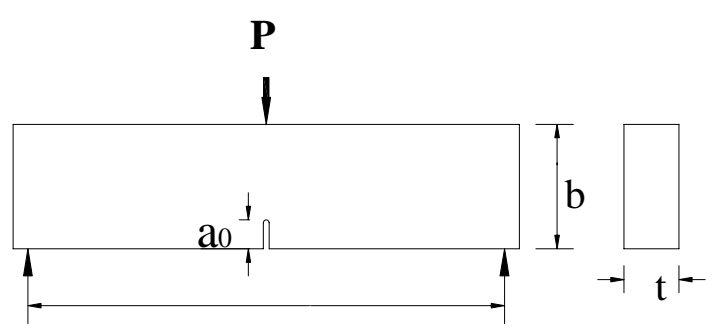

S

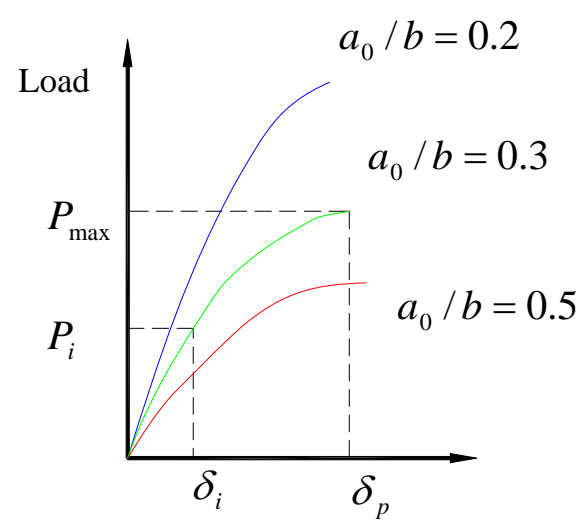

Deformation

(a)

(b)

Figure 2.18 Effective crack model

(Adopted from: Nallathambi and Karihaloo, 1989) 
Due to the existence of fracture process zone at the tip of the crack, when the load reaches the peak load $P_{\max }$ with a corresponding mid-span deflection $\delta_{p}$ as shown in Figure 2.18, the stiffness of the beam will differ from the original beam. In order to take into account this change in stiffness, a fictitious beam having a notch length $a_{e}$ and same stiffness as the real beam is introduced. To find the value of $a_{e}$, the following trial-anderror procedure can be applied:

1. Substituting the value of $P_{i}$ and corresponding displacement $\delta_{i}$ as well as notch length $a_{0}$ into the Eq. (2.12) to calculate the initial modulus of elasticity of the beam, say $E^{*}$.

2. Using the notched length $a_{0}$ as a starting value, substitute $a_{0}$ as well as $P_{\max }$ and deflection $\delta_{p}$ into Eq. (2.12) to calculate a new modulus of elasticity $E$. The $E$ will naturally be smaller than $E^{*}$.

3. Increasing the notch length by $\Delta a=0.01 a_{0}$, repeat the procedure in item 2 , until $E=E^{*} \pm 0.5 \%$. The final value of notch length is equal to $a_{e}$.

The critical stress intensity factor $K_{I c}{ }^{e}$ can then be calculated by the formulation from LEFM. The fracture in real concrete propagates in a stable way when the stress intensity factor and effective crack length reach the critical value, i.e.:

$$
K_{I}=K_{I c}^{e} \quad a=a_{e}
$$




\subsection{Test Methods for the Determination of Fracture Parameters}

Several test methods have been developed for the determination of the fracture properties of concrete during the last decade. However, till now, no test method has been adopted by ASTM as a standard fracture test for concrete. ASTM E 399 only covers the determination of the plane-strain fracture toughness $\left(K_{I C}\right)$ of metallic materials.

RILEM (The International Union of Testing and Research Laboratories for Materials and Structures) has been actively involved in various committees in developing test methods for analysis of cracked concrete structures. The work-of-fracture method (Hillerborg, 1976) was first recommended by RILEM in 1985 (RILEM 50 FMC, 1985), then another two methods: Jenq-Shah method and Bezant's size effect method were also proposed separately by RILEM in1990.

\subsubsection{Work-of - fracture method (RILEM 50 FMC)}

RILEM 50-FMC (1985) method is the first method proposed as a standard test for fracture properties of concrete. This method is the Hillerborg's fictitious crack model, which has been discussed in Section 2.3.3.2(a). The proposed standard uses a beam specimen loaded in three-point bending with a central edge notch, as shown in Figure 2.19. The dimension of the beam depends on the maximum size of the aggregate according to Table 2.1. The notch depth is equal to half of the beam depth $\pm 5 \mathrm{~mm}(0.2$ in).

The vertical load- point displacement should be recorded continuously during the test. The displacement should be measured with an accuracy of at least $0.01 \mathrm{~mm}$ (0.0004 in), and load should be measured with an accuracy of at least $2 \%$ of the maximum value. The 
rate should be approximately constant, and the maximum load can be reached within $60 \mathrm{~s}$ after the start of the test.

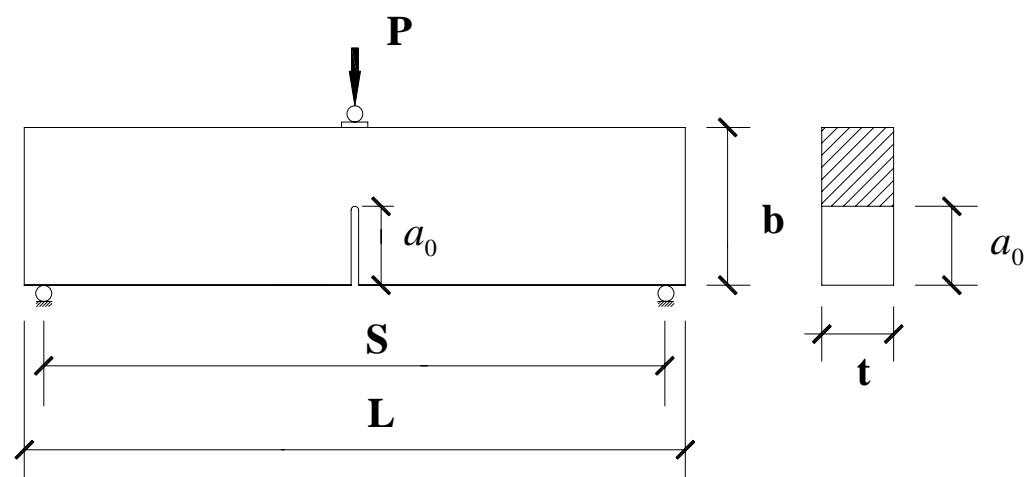

Figure 2.19 Hillerborg's three-point bending test (Adopted from: RILEM 50-FMC, 1985)

Table 2.1 Size of specimen for Hillerborg’s test (RILEM 50-FMC, 1985)

\begin{tabular}{c|c|c|c|c}
\hline $\begin{array}{c}\mathrm{D}_{\max } \\
(\mathrm{mm})\end{array}$ & $\begin{array}{c}\text { Depth d } \\
(\mathrm{mm})\end{array}$ & $\begin{array}{c}\text { Width t } \\
(\mathrm{mm})\end{array}$ & $\begin{array}{c}\text { Length L } \\
(\mathrm{mm})\end{array}$ & $\begin{array}{c}\text { Span S } \\
(\mathrm{mm})\end{array}$ \\
\hline $1-16$ & $100 \pm 5$ & $100 \pm 5$ & $840 \pm 10$ & $800 \pm 5$ \\
\hline $16.1-32$ & $200 \pm 5$ & $100 \pm 5$ & $1190 \pm 10$ & $1130 \pm 5$ \\
\hline $32.1-48$ & $300 \pm 5$ & $150 \pm 5$ & $1450 \pm 10$ & $1385 \pm 5$ \\
\hline $48.1-64$ & $400 \pm 5$ & $200 \pm 5$ & $1640 \pm 10$ & $1600 \pm 5$ \\
\hline
\end{tabular}

$$
(1 \mathrm{~mm}=0.0394 \mathrm{in})
$$

In order to get a complete load and loading point displacement curve, a closed-loop servo control testing system is recommended. Otherwise the test machine should be stiff enough to capture the descending part of the curve. The energy absorbed by the beam is represented by the area under the $P-\delta$ curve. The fracture energy per unit ligament can be calculated by:

$$
G_{F}=\frac{W_{0}+2 F_{1} \delta_{0}}{A_{\text {lig }}}
$$


where $W_{0}$ is the area under the measured $P-\delta$ curve; $F_{1}$ is the sum of self-weight; $\delta_{0}$ is the displacement at failure; $A_{\text {lig }}$ is the area of ligament.

\subsubsection{Jenq-Shah method}

RILEM recommendation (RILEM TC-89-FMT, 1990) covers the determination of the critical stress factor $K_{I c}{ }^{s}$ and $C T O D_{c}$ of mortar and concrete, using a three point bending beam. This method is based on the two parameters model by Jenq and Shah (1985).

Table 2.2 Size of specimen for two parameter test (RILEM TC-89-FMT, 1990)

\begin{tabular}{c|c|c|c|c}
\hline $\begin{array}{c}\mathrm{D}_{\max } \\
(\mathrm{mm})\end{array}$ & $\begin{array}{c}\text { Depth, d } \\
(\mathrm{mm})\end{array}$ & $\begin{array}{c}\text { Width, t } \\
(\mathrm{mm})\end{array}$ & $\begin{array}{c}\text { Length, L } \\
(\mathrm{mm})\end{array}$ & $\begin{array}{c}\text { Span, S } \\
(\mathrm{mm})\end{array}$ \\
\hline $1-25$ & $150 \pm 5$ & $80 \pm 5$ & $700 \pm 10$ & $600 \pm 5$ \\
\hline $25.1-50$ & $250 \pm 5$ & $150 \pm 5$ & $1100 \pm 10$ & $1000 \pm 5$ \\
\hline
\end{tabular}

$$
(1 \mathrm{~mm}=0.0394 \mathrm{in})
$$

The size of beam depends on the maximum aggregate size. Table 2.2 shows the dimension of the beam with different maximum size aggregate. The initial notch-to-depth ratio should be 1/3. A closed-loop testing system with the $C M O D$ as the feedback signal or a relatively stiff machine should be used to achieve a stable failure. Clip-on gauge is strongly recommended for the measurement of $C M O D$. As shown in Figure 2.17, the $C M O D$ and load should be continuously recorded, and the specimen should be unloaded at about $95 \%$ of the maximum load on the descending branch to obtain the unloading compliance $C_{u}$.

After knowing the initial compliance $C_{i}$ and unloading compliance $C_{u}$, the modulus of elasticity can be calculated from:

$$
E=\frac{6 S a_{0} g_{2}\left(\alpha_{0}\right)}{C_{i} b^{2} t}
$$


where $\alpha_{0}=\left(a_{0}+H_{0}\right) /\left(b+H_{0)}\right) ; S, b, t, a_{0}$, and $H_{0}$ are defined in Figure 2.17; $g_{2}$ is the geometric function. Assuming that the modulus of elasticity is constant, the effectiveelastic critical crack length $a_{c}$ can be calculated from

$$
a_{c}=\frac{C_{u} b^{2} t}{6 S g_{2}\left(\alpha_{c}\right)} E
$$

By substituting Eq. (2.15) into Eq. (2.16), and by using an iteration procedure, $a_{c}$ can be calculated from:

$$
\alpha_{c}=a_{0} \frac{C_{u} g_{2}\left(\alpha_{0}\right)}{C_{i} g_{2}\left(\alpha_{c}\right)}
$$

where $\alpha_{c}=\left(a_{c}+H_{0}\right) /\left(b+H_{0}\right)$

The $K_{\text {Ic }}{ }^{s}$ and $C T O D_{c}$ can be further calculated by the formulations from LEFM.

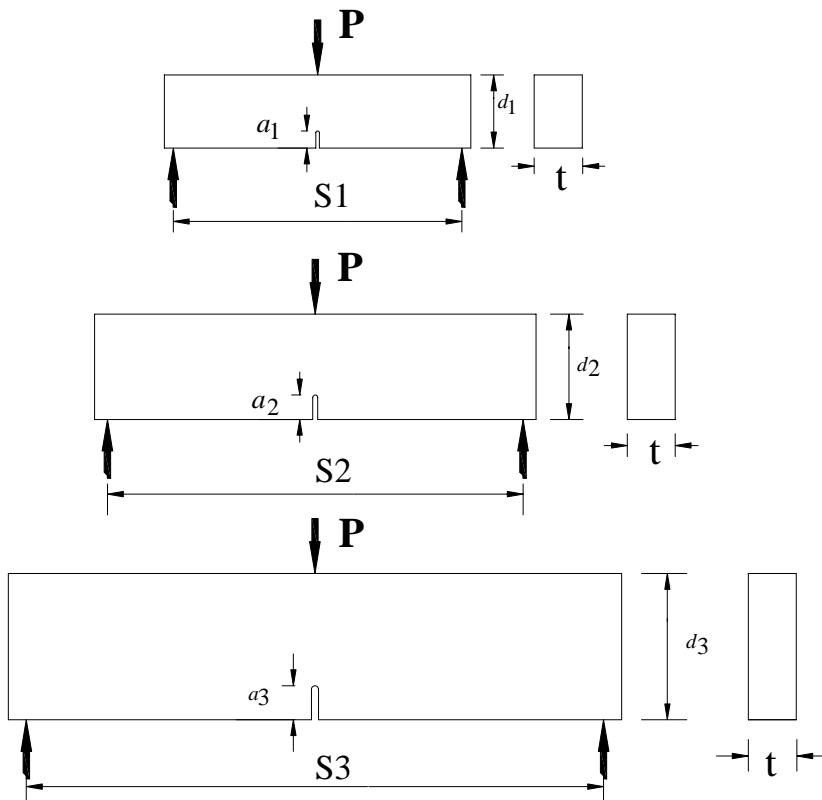

Figure 2.20 Bazant's size effect method (Adopted from: RILEM TC-89-FMT, 1990) 


\subsubsection{Size effect method}

Based on Bazant's size effect model, the following procedure is recommended by RILEM TC-89-FMT (1990).

Three-point bending beams are recommended for this method, the span-to-depth ratio ( $S / d$ ) should be at least 2.5; and the ratio of notch depth to the beam depth $\left(a_{0} / d\right)$ should be between $0.15 \sim 0.5$. As shown in Figure 2.20, specimens of at least three different sizes, characterized by the beam depths $b=b_{1}, b_{2}, b_{3} \ldots . . b_{n}$ and spans $S=S_{1}, S_{2}, S_{3} \ldots . . S_{n}$ must be tested. The smallest depth $b_{1}$ must not be larger than $5 d_{a}\left(d_{a}\right.$ is maximum aggregate size), and the largest $b_{n}$ must not be smaller than $10 d_{a}$. The choice of $\frac{b}{d_{a}}=3,6,12$, and 24 is preferable. The thickness of the beam should be kept constant.

An ordinary uniaxial testing machine without high stiffness is sufficient. However, a closed-loop control system is preferred for more consistent results. The loading rate should be such that the maximum load is reached within 5 minutes. Only the maximum loads $P_{1}, P_{2}, P_{i} \ldots P_{n}$ and corresponding specimens depths $b=b_{1}, b_{2}, b_{i} \ldots . . b_{n}$ are needed to determine the fracture parameters $G_{f}$ and $c_{f}$. The calculation procedure is as follows:

1. To calculate the maximum loads by considering the self-weight of the beams

$$
P_{i}^{0}=P_{i}+\frac{2 S_{i}-L_{i}}{2 S_{i}} g m_{i_{i}}
$$

where $L_{i}$ is the length of the beam; $g$ is the gravity acceleration; $m_{i}$ is the mass of the specimen; $P_{i}$ is maximum load, and $S_{i}$ is span. 
2. To plot $Y_{i}$ against $X_{i}$.

where: $Y_{i}=\left(\frac{t b_{i}}{P_{i}^{0}}\right)^{2}, X_{i}=b_{i}$; from a linear regression, the values of the slope $A_{B}$ and intercept $C_{B}$ can be obtained:

$$
A_{B}=\frac{\sum_{i=1}^{n}\left(X_{i}-\bar{X}\right)\left(Y_{i}-\bar{Y}\right)}{\sum_{i=1}^{n}\left(X_{i}-\bar{X}\right)^{2}} ; \quad C_{B}=\bar{Y}-A_{B} \bar{X}
$$

where: $\bar{X}=\frac{1}{n} \sum_{i=1}^{n} X_{i}, \bar{Y}=\frac{1}{n} \sum_{i=1}^{n} Y_{i}$

3. To determine the value of the material fracture energy $G_{f}$ from

$$
G_{f}=\frac{g\left(\alpha_{0}\right)}{E A_{B}}
$$

where $g\left(\alpha_{0}\right)$ is geometric function; $\alpha_{0}=a_{0} / b$, and $E$ is modulus of elasticity.

The length of fracture process zone for an infinitely large specimen $c_{f}$ can be further calculated by:

$$
c_{f}=\frac{g\left(\alpha_{0}\right)}{g^{\prime}\left(\alpha_{0}\right)}\left(\frac{C_{B}}{A_{B}}\right)
$$

where $g^{\prime}\left(\alpha_{0}\right)$ is the first order of $g\left(\alpha_{0}\right)$. 


\section{CHAPTER THREE}

\section{MATERIALS AND METHODS}

\subsection{Introduction}

This chapter describes the materials and methods for a total of 24 different HPC mixtures that were developed for the evaluation of fresh, hardened, and durability properties. These mixes were designed using limestone and gravel from different locations in West Virginia (WV), and the state-of-art HPC technologies were followed.

\subsection{Materials}

In this section, the detailed information of materials used in this research project is presented. These materials include cementitious materials, coarse and fine aggregates, and chemical admixtures.

\subsubsection{Cementitious Materials}

The cementitious materials used include: portland cement, fly ash, slag, silica fume, and metakaolin.

\section{(a) Portland Cement}

Commercially available Type I portland cement was used, conforming to AASHTO M85/ASTM 150. The specific gravity, specific surface area, and oxide compositions are listed in Table 3.1. 
Table 3.1 Basic properties and oxide compositions of cement, silica fume, fly ash, and metakaolin

\begin{tabular}{c|c|c|c|c}
\hline & $\begin{array}{c}\text { Portland } \\
\text { cement }\end{array}$ & $\begin{array}{c}\text { Silica } \\
\text { Fume }\end{array}$ & Fly Ash & Metakaolin \\
\hline Specific gravity & 3.15 & 2.18 & 2.47 & 2.78 \\
\hline $\begin{array}{c}\text { Specific surface, } \\
\left(\mathrm{m}^{2} / \mathrm{kg}\right)\end{array}$ & 385 & 21400 & 496 & 2526 \\
\hline Loss of ignition, \% & 1.2 & 1.64 & 3.00 & - \\
\hline $\mathrm{SiO}_{2}, \%$ & 20.7 & 100 & 49.34 & 52.35 \\
\hline $\mathrm{Al}_{2} \mathrm{O}_{3}, \%$ & 5.5 & - & 22.73 & 43.82 \\
\hline $\mathrm{CaO}_{0} \%$ & 63.6 & - & 3.09 & 0.10 \\
\hline $\mathrm{MgO}_{0} \%$ & 0.9 & - & 1.06 & 0.15 \\
\hline $\mathrm{SO}_{3}, \%$ & 2.7 & 0.156 & 0.97 & - \\
\hline $\mathrm{Na}_{2} \mathrm{O}, \%$ & 0.5 & 0.57 & 1.15 & - \\
\hline $\mathrm{K}_{2} \mathrm{O}, \%$ & - & - & 1.60 & 0.46 \\
\hline $\mathrm{TiO}_{2}, \%$ & - & - & - & - \\
\hline $\mathrm{MnO}_{0} \%$ & - & - & - & 1.42 \\
\hline $\mathrm{Fe}_{2} \mathrm{O}_{3}, \%$ & 3.6 & - & 16.01 & - \\
\hline
\end{tabular}

\section{(b) Fly ash}

Fly ash is a finely divided residue resulting from the combustion of ground or powdered coal. It is generally finer than cement and consists mainly of glassy-spherical particles. In ASTM C618, fly ash has been classified into two classes, F and C, based on the chemical composition of the fly ash. The fly ash used in this study was Class F conforming to AASHTO M295/ASTM C618. The specific gravity, specific surface area, and oxide compositions are listed in Table 3.1. 


\section{(c) Ground granulated blast-furnace slag}

Ground granulated blast furnace slag or simply slag is defined as a finely ground glassy granular material formed when molten blast-furnace slag is rapidly chilled. Slag is varying in size depending on its chemical composition and method of production as well as that of its iron source. The slag used in this project conformed to AASHTO M302 /ASTM C989-82. The specific gravity, specific surface area and compound compositions are listed in Table 3.2.

Table 3.2 Basic properties and compound compositions of slag

\begin{tabular}{c|c}
\hline & Slag \\
\hline Specific gravity & 2.88 \\
\hline Specific surface, $\mathrm{m}^{2} / \mathrm{kg}$ & 581 \\
\hline $\mathrm{C}_{3} \mathrm{~S}(\%)$ & 48 \\
\hline $\mathrm{C}_{2} \mathrm{~S}(\%)$ & 19 \\
\hline $\mathrm{C}_{3} \mathrm{~A}(\%)$ & 8 \\
\hline $\mathrm{C}_{4} \mathrm{AF}(\%)$ & 7.73 \\
\hline $\mathrm{Na}_{2} \mathrm{O}(\%)$ & 0.67 \\
\hline
\end{tabular}

\section{(d) Silica fume}

Silica fume is a by-product for producing silicon metal or ferrosilicon alloys. Because of its chemical and physical properties, it is a highly reactive pozzolan of very fine nature (about 100 times finer than cement particles), and is able to pack between cement particles. The silica fume used in this research project conformed to AASHTO M307 / ASTM C1240 and was commercially available. The specific gravity, specific surface area, and oxide compositions are listed in Table 3.1. 


\section{(e) Metakaolin}

Metakaolin is a white pozzolan made by calcining the kaolin clay under carefully controlled conditions to create an amorphous alumina silicate that is reactive in concrete. Same as silica fume, metakaolin is also a highly-reactivity pozzolan, and is able to pack between cement particles due to much smaller size (about 10 times finer than portland cement particle). Metakaolin is gaining more and more attention in concrete materials research due to its outstanding performance in enhancing the properties of the concrete. Metakaolin used in this study met the requirements of ASTM C618 Class N. The specific gravity, specific surface area, and oxide compositions are listed in Table 3.1.

\subsubsection{Aggregates}

Four different types of graded coarse aggregates were selected from 12 different sources as suggested by the WVDOH. One type of fine aggregate (sand) was selected from two different sources for this study. All the aggregates conformed to AASHTO M43 / ASTM C33.

The aggregates and the sources are as follows:

(1) Greer limestone with nominal maximum size $=25.4 \mathrm{~mm}$ (1 in), and specific gravity = 2.69, from Greer, WV (to be denoted as L1).

(2) Buckeye limestone with nominal maximum size $=25.4 \mathrm{~mm}$ (1 in), and specific gravity $=2.7$, from Blaney Hollow, WV (to be denoted as L2).

(3) Crushed gravel with nominal maximum size $=25.4 \mathrm{~mm}(1 \mathrm{in})$, and specific gravity $=$ 2.59, from Dulles Bottom, Joe Lucas Dredge, WV (to be denoted as G1).

(4) Gravel with nominal maximum size $=25.4 \mathrm{~mm}$ ( 1 in), and specific gravity $=2.56$, from Apple Grove plant (to be denoted as G2). 
(5) Fine aggregate (sand) with maximum nominal size of $4.76 \mathrm{~mm}$ (3/16 in), and specific gravity of 2.61, from Shelly Aggregate.

Table 3.3 lists the sieve analysis and basic physical properties for the four types of coarse aggregates. Table 3.4 and Table 3.5 list the sieve analysis, and basic properties of the sand respectively.

Table 3.3 Sieve analysis and basic properties of coarse aggregates

\begin{tabular}{|c|c|c|c|c|}
\hline & $\begin{array}{c}\text { Limestone \#1 } \\
\text { (L1) }\end{array}$ & $\begin{array}{c}\text { Limestone \#2 } \\
\text { (L2) }\end{array}$ & $\begin{array}{l}\text { Gravel \#1 } \\
\text { (G1) }\end{array}$ & $\begin{array}{l}\text { Gravel \#2 } \\
\text { (G2) }\end{array}$ \\
\hline $25.4 \mathrm{~mm}$ & 95 & 96 & 96 & 100 \\
\hline $19.0 \mathrm{~mm}$ & - & - & 76 & 89 \\
\hline $12.7 \mathrm{~mm}$ & 25 & 46 & 41 & 48 \\
\hline $9.5 \mathrm{~mm}$ & - & - & 17 & 20 \\
\hline $4.75 \mathrm{~mm}$ & 0 & 4 & 1 & 2 \\
\hline $2.36 \mathrm{~mm}$ & 0 & 2 & 1 & 1 \\
\hline SSD Specific Gravity & 2.68 & - & 2.59 & 2.56 \\
\hline Dry Specific Gravity & 2.69 & 2.70 & 2.54 & 2.51 \\
\hline $\begin{array}{c}\text { Apparent Specific } \\
\text { Gravity }\end{array}$ & 2.72 & - & 2.67 & 2.63 \\
\hline Absorption & $0.53 \%$ & $1.8 \%$ & $1.9 \%$ & $2.1 \%$ \\
\hline Source & Greer & Buckeye & $\begin{array}{l}\text { Joe Lucas } \\
\text { Dredge }\end{array}$ & Apple Grove \\
\hline
\end{tabular}

$(\mathrm{SSD}=$ Saturated surface dry)

$(1 \mathrm{~mm}=0.0394 \mathrm{in})$ 
Table 3.4 Sieve analysis of sand

\begin{tabular}{c|c}
\hline Sieve & Percentage Passing \\
\hline $4.75 \mathrm{~mm}$ & 97 \\
\hline $2.36 \mathrm{~mm}$ & 84 \\
\hline $1.18 \mathrm{~mm}$ & 72 \\
\hline $600 \mu \mathrm{m}$ & 57 \\
\hline $300 \mu \mathrm{m}$ & 18 \\
\hline $150 \mu \mathrm{m}$ & 4 \\
\hline $75 \mu \mathrm{m}$ & 2 \\
\hline
\end{tabular}

$(1 \mathrm{~mm}=0.0394 \mathrm{in})$

Table 3.5 Basic properties of sand

\begin{tabular}{c|c}
\hline SSD Specific Gravity & 2.61 \\
\hline Dry Specific Gravity & 2.59 \\
\hline Apparent Specific Gravity & 2.65 \\
\hline Absorption \% & 1.0 \\
\hline Source & Shelly \\
\hline
\end{tabular}




\subsubsection{Chemical admixtures}

Chemical admixtures constitute essential ingredients in concrete, in addition to portland cement, SCM, water, and aggregates. In this research, several different chemical admixtures were used to achieve desired HPC mixtures which have specific properties requirement such as: high slump, good workability, and low permeability. The following admixtures were used in this study.

1. Water reducing admixture (WRA)

This is a general use liquid WRA, which meets ASTM C494 requirements for: Type A water reducing, Type B retarding, and Type D water reducing and retarding admixture. The recommended usage for Type A application is 195-260 ml/100 kg of cementitious materials.

2. High-range water reducing admixture (HRWRA)

This is a naphthalene-based HRWRA conforming to ASTM C494 requirement for Type A water reducing admixture, and Type F high-range water reducing admixture. In this study, HRWRA was used as Type F application. The recommended usage for Type F application is $1000-1600 \mathrm{ml} / 100 \mathrm{~kg}$ of cementitious materials.

3. Air-entraining admixture (AEA)

This admixture is used for enhancing the durability of the concrete by entraining micro air bubbles within concrete. It meets the requirements of AASHTO M154/ ASTM C260. 


\subsection{Mixture Designs}

A total of 24 different mixture proportions were developed for laboratory evaluations. The mixture designs are based on the following considerations:

1. WVDOH Class $\mathrm{H}$ concrete and the ready mix concrete plant practice. Several mixture proportions were collected from different ready mix plants with the help of the WVDOH personnel. The laboratory trials were conducted on different batches based on ready mix plant mixtures as well as WVDOH Class $\mathrm{H}$ specification (WVDOH, 2003). For Class H concrete, the fly ash + silica fume and slag + silica fume mixtures with w/cm of 0.4 were initially designed satisfying the cement and SCM quantities prescribed in the specifications. The fly ash content was varied by $5 \%$ after trials; the fly ash obtained from a power station was found to vary in quality from time to time. Trials were conducted on different batches with fly ash supplies as follows: (1) mixture similar to $\mathrm{DOH}$, (2) mixture with 5\% higher fly ash than $\mathrm{DOH}$, and (3) mixture with 5\% less fly ash than DOH. Table 3.6 and Table 3.7 provide comparisons of SCM combinations among WVDOH Class $\mathrm{H}$ concrete, ready mix plant mixtures, and proposed mixtures in this research.

2. Ternary combination of the SCM (slag + fly ash + silica fume) was included as an approach to reduce portland cement quantity in the concrete. Table 3.8 shows the SCM combinations as percentage of total cementitious materials.

3. Metakaolin as a relatively new material was used to develop HPC mixtures applicable to the state of WV for its highly pozzolanic and packing properties. Metakaolin was also known for its increasing application in other state DOT for bridge structures. 
4. The w/cm ratios of 0.35 and 0.3 were included in this study to examine the performance of HPC with lower w/cm.

As shown in Figure 3.1, for each type of aggregate, four different mixture proportions (different SCM combinations) were prepared with the $\mathrm{w} / \mathrm{cm}=0.4$. For the $\mathrm{w} / \mathrm{cm}=0.35$ and 0.3, only Limestone1 (L1) aggregate was used to prepare the mixtures. Table 3.9 and Table 3.10 list the mixture proportions of HPC with $\mathrm{w} / \mathrm{cm}=0.4$, and $\mathrm{w} / \mathrm{cm}=0.35$ and 0.3 , respectively.

Table 3.6 Overview of various fly ash + silica fume mixtures

\begin{tabular}{c|c|c|c|c|c|c|c|c|c}
\hline \multirow{2}{*}{ w/cm } & \multicolumn{3}{|c|}{$\begin{array}{c}\text { Proposed Mixture } \\
\left(\mathrm{kg} / \mathrm{m}^{3}\right)\end{array}$} & \multicolumn{2}{c|}{$\begin{array}{c}\text { WVDOH Spec. of } \\
\text { Class H }\left(\mathrm{kg} / \mathrm{m}^{3}\right)\end{array}$} & \multicolumn{2}{c}{$\begin{array}{c}\text { Typical Ready Mix Plant } \\
\left(\mathrm{kg} / \mathrm{m}^{3}\right)\end{array}$} \\
\cline { 2 - 9 } & Cement & Fly ash & $\begin{array}{c}\text { Silica } \\
\text { fume }\end{array}$ & Cement & Fly ash $\begin{array}{c}\text { Silica } \\
\text { fume }\end{array}$ & Cement & Fly ash & $\begin{array}{c}\text { Silica } \\
\text { fume }\end{array}$ \\
\hline \multirow{2}{*}{0.4} & 285.6 & 72.6 & 19.0 & 278.9 & 97.6 & 17.8 & 278.9 & 68.8 & 17.8 \\
\hline
\end{tabular}

$$
\left(1 \mathrm{~kg} / \mathrm{m}^{3}=1.685 \mathrm{lb} / \mathrm{yd}^{3}\right)
$$

Table 3.7 Overview of various slag + silica fume mixtures

\begin{tabular}{c|c|c|c|c|c|c|c|c|c}
\hline \multirow{2}{*}{$\mathrm{w} / \mathrm{cm}$} & \multicolumn{3}{|c|}{$\begin{array}{c}\text { Proposed Mixture } \\
\left(\mathrm{kg} / \mathrm{m}^{3}\right)\end{array}$} & \multicolumn{2}{c|}{$\begin{array}{c}\text { WVDOH Spec. of } \\
\text { Class H }\left(\mathrm{kg} / \mathrm{m}^{3}\right)\end{array}$} & \multicolumn{2}{|c}{$\begin{array}{c}\text { Typical Ready Mix Plant } \\
\left(\mathrm{kg} / \mathrm{m}^{3}\right)\end{array}$} \\
\cline { 2 - 9 } & Cement & Slag & $\begin{array}{c}\text { Silica } \\
\text { fume }\end{array}$ & Cement & Slag & $\begin{array}{c}\text { Silica } \\
\text { fume }\end{array}$ & Cement & Slag & $\begin{array}{c}\text { Silica } \\
\text { fume }\end{array}$ \\
\hline \multirow{2}{*}{0.4} & 250.0 & 116.0 & 19.0 & 251.0 & 125.5 & 17.8 & 251.0 & 111.0 & 17.8 \\
\hline
\end{tabular}

$$
\left(1 \mathrm{~kg} / \mathrm{m}^{3}=1.685 \mathrm{lb} / \mathrm{yd}^{3}\right)
$$


Table 3.8 Mixture types and percentage of SCM used

\begin{tabular}{c|c|c|c|c|c|c}
\hline \multirow{2}{*}{$\begin{array}{c}\text { SCM } \\
\text { Combinations }\end{array}$} & \multicolumn{2}{|c|}{ SCM ( \% of total cementitious materials) } & \multirow{2}{*}{$\begin{array}{c}\text { Total SCM } \\
\text { (\%) }\end{array}$} & $\begin{array}{c}\text { Cement (\% of } \\
\text { total cementitious } \\
\text { materials) }\end{array}$ \\
\cline { 2 - 5 } & $\begin{array}{c}\text { Silica- } \\
\text { fume }\end{array}$ & Fly ash & Slag & Metakaolin & 35 & 65 \\
\hline Slag + Silica fume & 5 & - & 30 & - & 25 & 75 \\
\hline $\begin{array}{c}\text { Fly ash + } \\
\text { Silica fume }\end{array}$ & 5 & 20 & - & - & 45 & 55 \\
\hline $\begin{array}{c}\text { Slag + Fly ash + } \\
\text { Silica fume }\end{array}$ & 5 & 15 & 25 & - & 10 & 90 \\
\hline Metakaolin & & & & 10 & \\
\hline
\end{tabular}

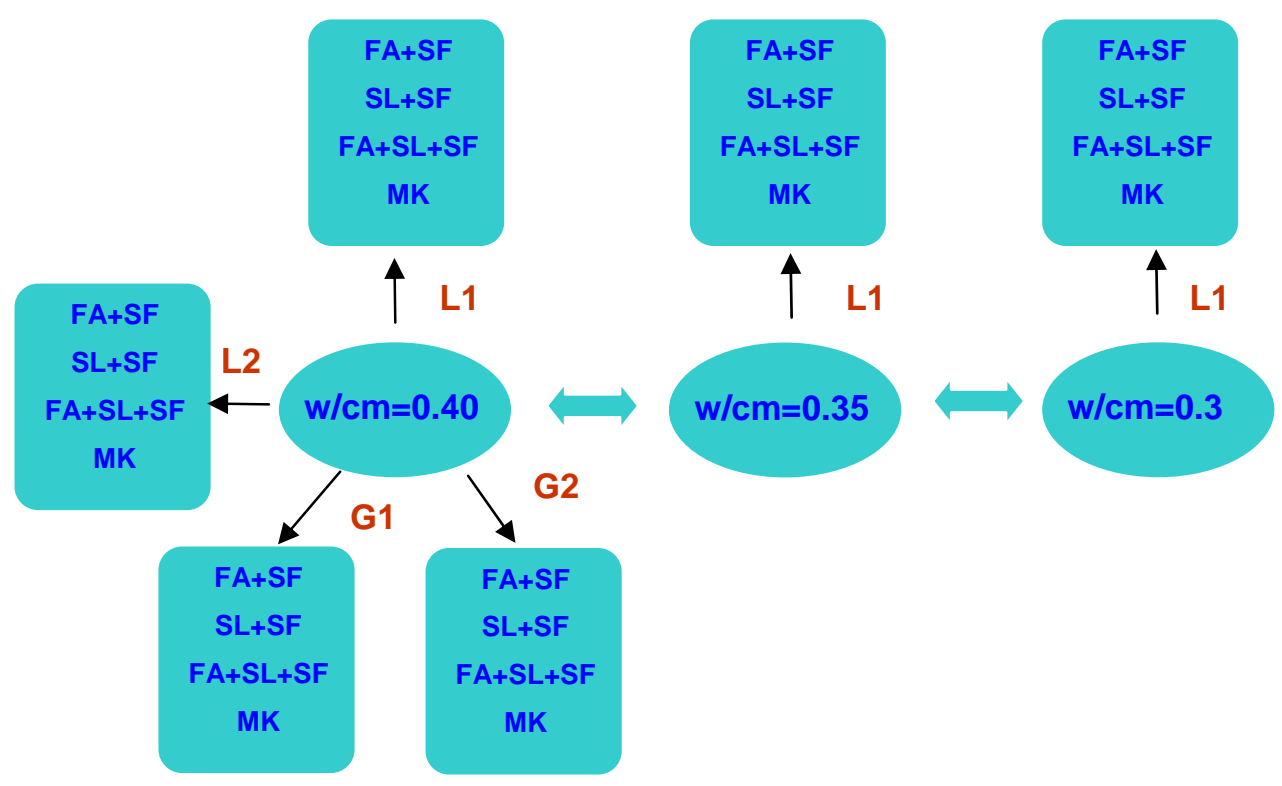

Note: $\quad$ FA+ SF : fly ash + silica fume; SL+ SF: slag + silica fume;

FA+ SL+ SF: fly ash + slag + silica fume; MK: metakaolin.

Figure 3.1 Mixture design scheme 
Table 3.9 Mixture proportions of HPC with $\mathrm{w} / \mathrm{cm}=0.4$

\begin{tabular}{|c|c|c|c|c|c|c|c|c|c|c|}
\hline Mixtures & $\begin{array}{c}\text { Cement } \\
\left(\mathrm{kg} / \mathrm{m}^{3}\right)\end{array}$ & $\begin{array}{l}\text { Slag } \\
(\mathrm{kg} / \mathrm{m} 3)\end{array}$ & $\begin{array}{c}\text { Fly } \\
\text { ash } \\
\left(\mathrm{kg} / \mathrm{m}^{3}\right)\end{array}$ & $\begin{array}{l}\text { Silica } \\
\text { fume } \\
\left(\mathrm{kg} / \mathrm{m}^{3}\right)\end{array}$ & $\begin{array}{l}\text { Meta- } \\
\text { Kaolin } \\
\left(\mathrm{kg} / \mathrm{m}^{3}\right)\end{array}$ & $\begin{array}{l}\text { Sand } \\
\left(\mathrm{kg} / \mathrm{m}^{3}\right)\end{array}$ & $\begin{array}{c}\text { Coarse } \\
\text { Aggregate } \\
\left(\mathrm{kg} / \mathrm{m}^{3}\right)\end{array}$ & $\mathbf{w} / \mathbf{c m}$ & $\begin{array}{l}\text { Slump } \\
(\mathrm{mm})\end{array}$ & $\begin{array}{c}\text { Air } \\
\text { Content } \\
(\%)\end{array}$ \\
\hline SL-SF-L1-0.4 & 250.0 & 116.0 & & 19.0 & & 699 & 1038 & 0.4 & 178 & 6.0 \\
\hline FA-SF-L1-0.4 & 285.6 & & 76.2 & 19.0 & & 699 & 1038 & 0.4 & 152 & 6.0 \\
\hline SL-FA-SF-L1-0.4 & 208.2 & 94.5 & 56.7 & 19 & & 699 & 1038 & 0.4 & 178 & 7.0 \\
\hline MK-L1-0.4 & 352.0 & & & & 39.3 & 699 & 1038 & 0.4 & 191 & 6.4 \\
\hline SL-SF-L2-0.4 & 250.0 & 116.0 & & 19.0 & & 696 & 1044 & 0.4 & 178 & 7.0 \\
\hline FA-SF-L2-0.4 & 285.6 & & 76.2 & 19.0 & & 696 & 1044 & 0.4 & 178 & 6.0 \\
\hline SL-FA-SF-L2-0.4 & 208.2 & 94.5 & 56.7 & 19 & & 696 & 1044 & 0.4 & 165 & 6.8 \\
\hline MK-L2-0.4 & 352.0 & & & & 39.3 & 696 & 1044 & 0.4 & 203 & 6.0 \\
\hline SL-SF-G1-0.4 & 250.0 & 116.0 & & 19.0 & & 687 & 1018 & 0.4 & 178 & 6.8 \\
\hline FA-SF-G1-0.4 & 285.6 & & 76.2 & 19.0 & & 687 & 1018 & 0.4 & 152 & 5.8 \\
\hline SL-FA-SF-G1-0.4 & 208.2 & 94.5 & 56.7 & 19 & & 687 & 1018 & 0.4 & 152 & 6.0 \\
\hline MK-G1-0.4 & 352.0 & & & & 39.3 & 687 & 1018 & 0.4 & 203 & 7.0 \\
\hline SL-SF-G2-0.4 & 250.0 & 116.0 & & 19.0 & & 673 & 1011 & 0.4 & 203 & 7.6 \\
\hline FA-SF-G2-0.4 & 285.6 & & 76.2 & 19.0 & & 673 & 1011 & 0.4 & 203 & 7.0 \\
\hline SL-FA-SF-G2-0.4 & 208.2 & 94.5 & 56.7 & 19 & & 673 & 1011 & 0.4 & 216 & 8.0 \\
\hline MK-G2-0.4 & 352.0 & & & & 39.3 & 673 & 1011 & 0.4 & 178 & 7.0 \\
\hline
\end{tabular}


Table 3.10 Mixture proportions of HPC with $\mathrm{w} / \mathrm{cm}=0.35$ and 0.3

\begin{tabular}{|c|c|c|c|c|c|c|c|c|c|c|}
\hline Mixtures & $\begin{array}{c}\text { Cement } \\
\left(\mathbf{k g} / \mathbf{m}^{3}\right)\end{array}$ & $\begin{array}{l}\text { Slag } \\
(\mathrm{kg} / \mathrm{m} 3)\end{array}$ & $\begin{array}{c}\text { Fly ash } \\
\left(\mathbf{k g} / \mathbf{m}^{3}\right)\end{array}$ & $\begin{array}{l}\text { Silica } \\
\text { fume } \\
\left(\mathrm{kg} / \mathrm{m}^{3}\right)\end{array}$ & $\begin{array}{c}\text { Meta- } \\
\text { Kaolin } \\
\left(\mathrm{kg} / \mathbf{m}^{3}\right)\end{array}$ & $\begin{array}{l}\text { Sand } \\
\left(\mathrm{kg} / \mathrm{m}^{3}\right)\end{array}$ & $\begin{array}{c}\text { Coarse } \\
\text { Aggregate } \\
\left(\mathbf{k g} / \mathbf{m}^{3}\right)\end{array}$ & $\mathbf{w} / \mathbf{c m}$ & $\begin{array}{l}\text { Slump } \\
\text { (mm) }\end{array}$ & $\begin{array}{c}\text { Air } \\
\text { Content } \\
(\%)\end{array}$ \\
\hline SL-SF-L1-0.35 & 292.5 & 135 & & 22.5 & & 672 & 999 & 0.35 & 191 & 6.4 \\
\hline FA-SF-L1-0.35 & 334.0 & & 88.7 & 22.2 & & 672 & 999 & 0.35 & 203 & 6.0 \\
\hline SL-FA-SF-L1-0.35 & 243.0 & 110.4 & 66.2 & 22.1 & & 672 & 999 & 0.35 & 203 & 7.0 \\
\hline MK-L1-0.35 & 411.8 & & & & 46.2 & 672 & 999 & 0.35 & 178 & 7.0 \\
\hline SL-SF-L1-0.3 & 334.8 & 154.5 & & 25.8 & & 632 & 987 & 0.3 & 191 & 6.4 \\
\hline FA-SF-L1-0.3 & 381.5 & & 101.7 & 25.4 & & 632 & 987 & 0.3 & 203 & 6.0 \\
\hline SL-FA-SF-L1-0.3 & 277.6 & 126.1 & 75.5 & 25.2 & & 632 & 987 & 0.3 & 203 & 7.0 \\
\hline MK-L1-0.3 & 472.4 & & & & 52.5 & 632 & 987 & 0.3 & 178 & 7.0 \\
\hline
\end{tabular}

\section{Note:}

- Mixtures contained 2.46 to $2.8 \mathrm{~L} / \mathrm{m}^{3}$ of HRWRA, 0.62 to $0.75 \mathrm{~L} / \mathrm{m}^{3}$ of WRA, and 0.85 to 1.35 $\mathrm{L} / \mathrm{m}^{3}$ of AEA for various mixtures to achieve the desired slump and air content.

- $\mathrm{SL}=$ slag; $\mathrm{SF}=$ silica fume; $\mathrm{FA}$ = fly ash; $\mathrm{MK}=$ metakaolin .

- $\quad$ L1 = Greer limestone; L2 = Buckey limestone; G1 = Joe Lucas gravel; G2 = Apple Groove gravel.

- $\quad 1 \mathrm{~kg} / \mathrm{m}^{3}=1.685 \mathrm{lb} / \mathrm{yd}^{3}$. 


\subsection{Testing Organization}

The testing program includes preparation of concrete, curing, testing for fresh properties and hardened mechanical properties, free and restrained shrinkage test, and fracture mechanics test.

\subsubsection{Preparation of concrete}

Mixing procedures were based on guidelines and sequences recommended by ready mix plants and admixture suppliers, with necessary modifications for laboratory preparation based on relevant AASHTO and ASTM standards. The detailed mixture sequences are listed as follows:

1. Batched the materials by weight;

2. Added the AEA to the sand and WRA to the water, and stirred thoroughly;

3. Cleaned and wetted the mixer;

4. Added the coarse aggregate, 3/4 of the mixing water with the solution of WRA, and mixed them;

5. Added silica fume or metakaolin (as the case may be) to the mixer, followed by rotation of the mixer for 1 minute;

6. Added fine aggregate to mixer, and mixed it for another 1 minute;

7. Added cementitious material (cement, slag or fly ash) and remaining water and mixed for about 3 minutes;

8. Allowed 3-mimutes rest, which was followed by a 2-minute final mixing;

9. Added the HRWRA, and mixer was rotated till the target slump was achieved.

All the specimens were cast in accordance with AASHTO T126 / ASTM C192, “Standard Practice for Making and Curing Concrete Test Specimen in Laboratory” or 
AASHTO PP 34-99 “Standard Practice for Estimating the Cracking Tendency of Concrete.” All mixtures were prepared in two batches to account for the variations of batching, mixing, placing, and curing of concrete in the laboratory.

\subsubsection{Curing}

Immediately after casting (within 3 5 minutes), the molded specimens were covered with wet burlaps for about 24 hours at room temperature. After demolding, the specimens for direct tensile test, free shrinkage test, fracture test, and ring test were removed to a environmental chamber especially built for this project, which is capable of controlling the temperature and humidity automatically. The temperature and humidity of the chamber were set a $23{ }^{\circ} \mathrm{C}\left(73^{\circ} \mathrm{F}\right)$ and $50 \%$ relative humidity, respectively. The specimens for compressive strength test and modulus of elasticity test were cured under lime water at $23 \pm 2{ }^{\circ} \mathrm{C}\left(73 \pm 3^{\circ} \mathrm{F}\right)$ until testing age.

\subsubsection{Testing of fresh properties}

The fresh properties were measured using the following standard methods:

AASHTO T119/ASTM C143 for slump;

AASHTO T121/ASTM C138 for unit weight;

AASHTO T152/ASTM C231 for air content by pressure method.

The slump was measured within $5 \sim 20$ minutes after initial contact of water with cementitious materials in order to avoid major slump loss. The unit weight was measured to estimate fresh density and yield of concrete. The total air content was measured to ensure adequate air-entrainment. 


\subsubsection{Testing of mechanics properties}

The hardened mechanical properties tested are: compressive strength, modulus of elasticity, Poisson's ratio, and direct tensile strength.

\subsubsection{Compressive strength test}

AASHTO T22 / ASTM C39 were followed for the compressive strength test. For every mixture, two batches were cast, and every batch had two replicate cylinder specimens of $101.6 \mathrm{~mm}$ (4 in) diameter $\times 203.2 \mathrm{~mm}$ (8 in) long for each age of 1-day, 3day, 7-day, 14-day, 28-day and 90-day. The testing was conducted using a hydraulic machine at a constant load rate of $2.5 \mathrm{kN} / \mathrm{s}(557 \mathrm{lb} / \mathrm{s})$.

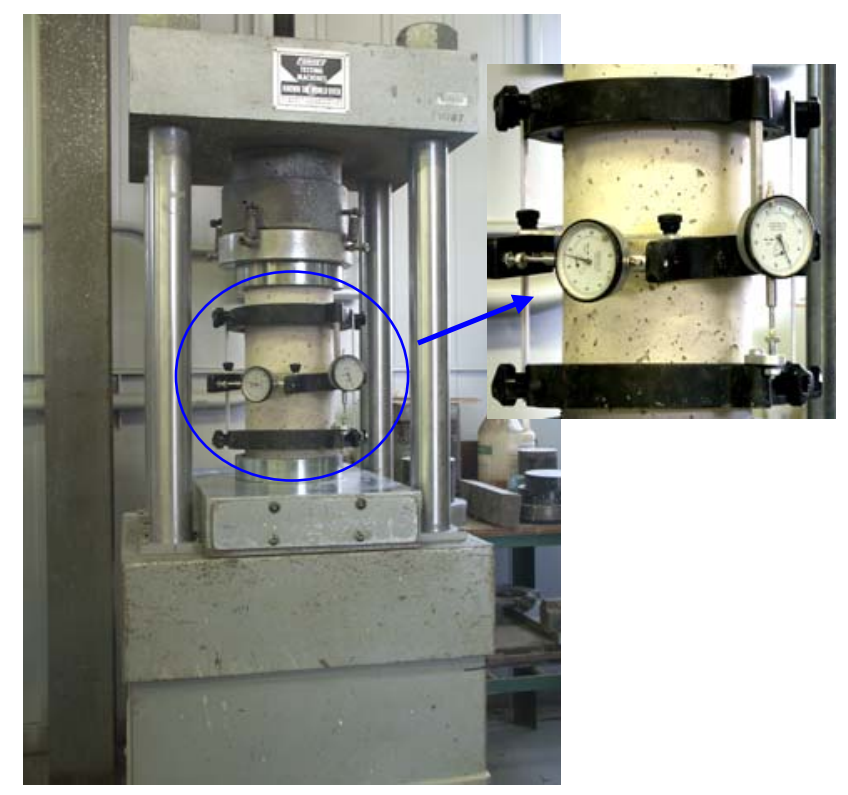

Figure 3.2 Static modulus of elasticity test in progress

\subsubsection{Modulus of elasticity test}

Static modulus of elasticity was tested per ASTM C469. Same as compressive strength test, for every mixture two batches were cast, and every batch had three replicate cylinder specimens of $152.4 \mathrm{~mm}$ (6 in) diameter $\times 304.8 \mathrm{~mm}$ (12 in) long. 28-day static 
modulus of elasticity and Poisson's ratio were measured. Figure 3.2 shows the static modulus of elasticity and Poisson's ratio tests in progress.

\subsubsection{Direct tensile test}

To date, no standard is available for direct tensile test due to considerable technical problems encountered such as: eccentricity of loading, improper failure of the specimen, inconsistency of results due to notch-sensitivity of concrete and size effect. In this study, direct tensile test was conducted by modifying few published works (USB 4914-92; Wang et al., 1990; and Li and Ansari, 2000). The whole test setup is shown in Figure 3.3, and the test details are as follows:

1. The size of the cylinder specimen is $101.6 \mathrm{~mm}$ (4 in) in diameter $\times 203.4 \mathrm{~mm}(8$ in) in length.

2. The specimen was attached to upper and lower grips of an MTS machine through a "link-chain" containing three rings, as shown in Figure 3.3.

3. The specimen was glued to steel end-plates with a commercially available and general purpose high performance epoxy. The steel plates had the same cross section as the concrete cylinder, and had threaded holes at their centers to attach the bottom chain-rings.

4. The specimen had $31.75 \mathrm{~mm}$ (1.25 in) deep notches at the mid-height symmetrically on both sides, as shown in Figure 3.3. The notches were cut using a diamond-blade saw.

The loading link-chain made the application of a concentric load possible, and the high performance epoxy and mid-height notch made possible the failure of the specimen through the notched section of the specimen. 


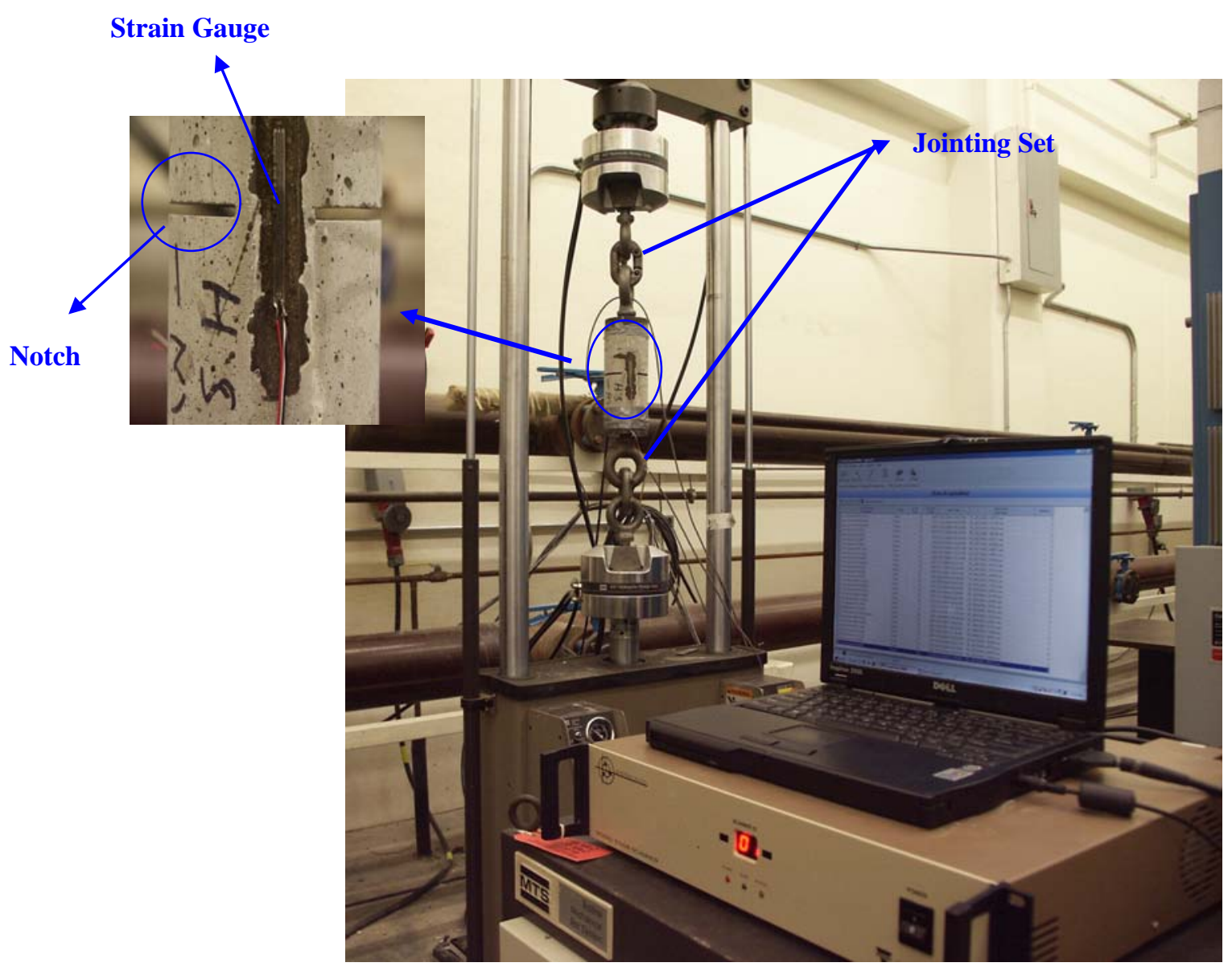

Figure 3.3 Direct tensile strength test setup

For each selected mixture, three replicate cylinder specimens of $101.6 \mathrm{~mm}$ (4 in) in diameter $\times 203.4 \mathrm{~mm}$ (8 in) in length were cast for each age. The ages of testing were 3, 7, 14, 28, and 90-day. The tensile test specimens and restrained shrinkage ring specimens were made from the same batch in order to keep the data comparable. All the specimens were cured in an environmental chamber with the condition of $23^{\circ} \mathrm{C}\left(73^{\circ} \mathrm{F}\right)$, and $50 \%$ relative humidity. Approximately 12 hours prior to the testing age, the specimens were taken out of the chamber, and the ends were smoothed with a grinding machine. In order 
to achieve perfect alignment, every specimen was treated carefully while gluing the steel plates on it. The epoxy required about 12 hours for $100 \%$ strength curing at room temperature.

Two strain gauges of $75 \mathrm{~mm}$ (3 in) long were longitudinally bonded on the unnotched section, on opposite sides (Figure 3.3), for evaluating the maximum strain and tensile modulus of elasticity. The test was conducted using a MTS machine connected to an external data acquisition system, which recorded the load and deformation automatically. The specimens were tested under a displacement control mode with a displacement rate of $0.0015 \mathrm{~mm} / \mathrm{s}(0.0006 \mathrm{in} / \mathrm{s})$, till the failure of the specimen. Testing of each specimen was completed between 18 - 20 minutes.

\subsubsection{Free and restrained shrinkage test}

\subsubsection{Free shrinkage test}

Free shrinkage test was conducted per AASHTO T160 / ASTM C157. The curing condition was the same as for the ring specimen used for cracking study. For every selected mixture, five $76.2 \mathrm{~mm} \times 76.2 \mathrm{~mm} \times 311.2 \mathrm{~mm}(3 \mathrm{in} \times 3$ in $\times 11.75 \mathrm{in})$ prisms were cast with the same batch concrete as for ring specimens. Two of the five specimens were partially sealed with silicon sealers leaving a same exposure surface to volume ratio as ring specimens. After one day curing under the wet burlap, the prisms were demolded and removed to the environmental chamber, with the temperature of $23{ }^{\circ} \mathrm{C}\left(73^{\circ} \mathrm{F}\right)$ and relative humidity of $50 \%$. The onset of drying after one day was selected to capture high shrinkage values.

A digital length comparator was used to measure the length change of the prisms as shown in Figure 3.4. Directly after demolding, the specimens were measured to obtain 
the initial value. After this, the readings were taken every 24 hours for the first 14 days, and then every 3 days until 28-day, followed by every 10 days till 90-day. The average value was used as the free shrinkage strain of the specimen.

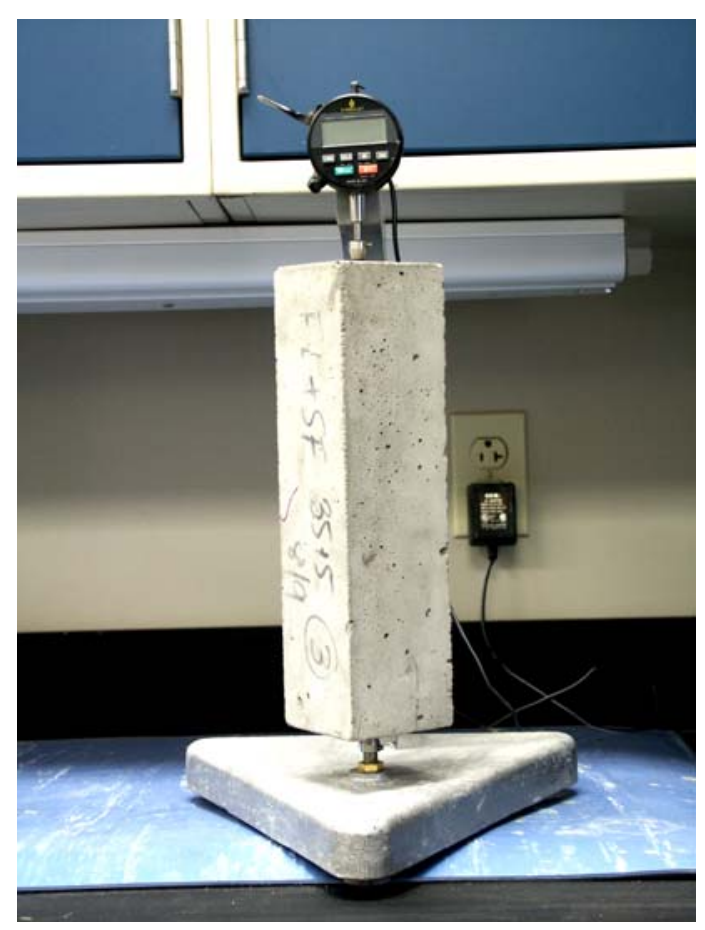

Figure 3.4 Free shrinkage test setup

\subsubsection{Restrained shrinkage test}

The restrained shrinkage test was conducted per AASHTO PP34-99 "Standard Practice for Estimating the Cracking Tendency of Concrete.” The details are discussed in Chapter Five.

\subsubsection{Fracture mechanics test}

In this study, Hillerborg's three-point bending beam method was employed to study the fracture properties of the selected HPC mixtures. The details are discussed in Chapter Seven. 


\section{CHAPTER FOUR}

\section{PROPERTIES OF FRESH AND HARDENED HPC}

\subsection{Introduction}

In this chapter, fresh properties, and hardened properties that included compressive strength and modulus of elasticity of all 24 different HPC mixtures were evaluated and compared. Based on the experimental results of these tests as well as the results from durability tests conducted by Fan (Fan, 2005) as part of the comprehensive studies on HPC, 18 mixtures were selected for further study. Direct tensile strength, free shrinkage and restrained shrinkage cracking of these 18 mixtures were evaluated and compared. The experimental results of direct tensile strength and free shrinkage tests will be discussed in this chapter. The restrained shrinkage properties will be discussed in the following chapter (Chapter Five).

\subsection{Fresh Properties}

\subsubsection{Significance of the test}

Slump, air content and unit weight are the basic fresh properties of concrete, which are essential for evaluation of any concrete. These properties are greatly influenced in HPC mixtures by the presence of various SCM and chemical admixtures. The specification for WVDOH Class H concrete (WVDOH, 2003) prescribes slump values before and after addition of HRWRA as $102 \mathrm{~mm}$ (4 in) and 178 mm (7 in), respectively. The target entrained air content is specified as $6.5 \pm 1.5 \%$. In the present research, 
slumps were measured for all HPC produced in order to check whether above slump criteria are reasonable with current mixtures, or alternate slump criteria are required. Air content tests were also conducted for the same purpose.

\subsubsection{Results and discussions}

Mix design was done in such a way that all the mixtures could be produced in the laboratory without any difficulty. From Table 3.8, it is observed that SCM can be used from $10 \%$ to $45 \%$ to produce reasonable fresh concrete properties. Also in case of concrete with fly ash and slag, the silica fume could be used judiciously to compensate the limitations of using slag and fly ash alone. The fresh properties of all the 24 HPC mixtures are listed in Table 3.9 and Table 3.10. In general, all HPC batched was found to be free from segregation with some variation in the degree of cohesiveness. Mixtures with metakaolin were observed to be of lighter color, or "whitish” in appearance than the other mixtures. Mixtures with w/cm as low as 0.3 were also acceptable, though higher quantity of cementitious materials and HRWRA were required to improve the quality of mixtures.

The initial slumps (before addition of HRWRA) were $25 \mathrm{~mm}$ to $50 \mathrm{~mm}$ (1 to $2 \mathrm{in}$ ) (not shown in Tables 3.9 and 3.10) for all mixtures. The use of various SCM, particularly silica fume or metakaolin, and low w/cm could not achieve a 102 mm (4 in) slump without adding HRWRA to conform to WVDOH specification. An attempt was made to increase the initial slump by increasing the quantity of WRA (which was usually added at the beginning of the mix), but this did not work as it caused difficulty in demolding and entrained excessive air. However, after HRWRA was added, the $152 \mathrm{~mm}$ to $203 \mathrm{~mm}$ (6 to 
8 in) slump could be achieved for all mixtures (even mixtures with initial slump of 25 $\mathrm{mm}$, or 1 in).

The air contents were found to be between 5.8 to $8.0 \%$. The results agreed well with the requirements for HPC produced by other state DOTs, researchers (Christopher, 1999 and Kerkhoff, 2002) and Class H concrete of WVDOH.

Concrete with gravel G2 exhibited slightly higher slump and air content compared to mixtures with other aggregates at same level of HRWRA. This may be due to gravel G2 being slightly crushed as compared to gravel G1 and limestone aggregates. A little bleeding was observed for fly ash + silica fume (FA-SF) and slag + silica fume (SL-SF) mixtures with limestone L2. However, this may be considered beneficial as the HPC to be used in the bridge decks can prevent plastic shrinkage cracking if it has controlled bleeding.

\subsection{Compressive Strength and Modulus of Elasticity}

\subsubsection{Significance of the test}

Compressive strength is the single most important property for the evaluation of concrete mixtures as all material and design specification are based on this parameter. In this project, the compressive strength tests were conducted at different ages to monitor the strength development of HPC with time. This is important because the early age strength can guide the contractor on finishing and curing, while the latter strength is a measure of long-term performance. The compressive strength test results of different mixtures up to 90 days enabled to compare the relative strength development of all mixture designs. Further studies will explain how the compressive strength data needs to 
be considered along with modulus of elasticity and free shrinkage in order to predict the cracking of concrete.

As discussed in Chapter One, for bridge decks, the early age cracking due to shrinkage is the most common type of failure. The cracking is influenced by both material properties such as tensile strength, modulus of elasticity, fracture energy, free shrinkage, creep, and structural properties such as degree of restraining effect on deck. So it is important to determine the static modulus of elasticity of HPC for application to bridge decks.

\subsubsection{Results and discussions}

\subsubsection{Compressive strength}

Figure 4.1 (a) through (f) display the compressive strength developments up to 90 days for all 24 mixtures. The testing data of compressive strength are listed in Appendix A. From the results the following observations are made:

1. HPC with Metakaolin (MK) exhibited the highest compressive strength up to 90 days in all cases, though, at 90 days, the strength of SL-SF was almost equal to MK for HPC with gravel. MK also improved early age strength more than any other SCM. Similar results can be found in the investigations conducted by Caldarone et al. (1994) and Ding and Li (2002). Wild et al. (1996) identified three elementary factors which contributed to the compressive strength development of MK concrete. Therefore: filler effect, the acceleration of portland cement hydration, and the pozzolanic reaction which has its maximum effect between 714 days. Another reason may be that for MK, the replacement level was least compared to other SCM additions. 
2. Among the mixtures, slag + fly ash + silica fume (SL-FA-SF) mixtures showed the lowest strength at all ages for all aggregates used. The SL-SF and FA-SF mixtures were found to lie in between MK and SL-FA-SF mixtures. Comparison between SL-SF and FA-SF mixtures showed that the former one performed better than the latter due to hydraulic nature of slag. However, the early age (till 14 days) strength of SL-SF mixtures was lower than that of the FA-SF mixtures due to the higher usage of SCM, which possibly slowed the rate of hydration reaction.

3. The use of ternary system (cement + fly ash + silica fume or cement + slag + silica fume) has been established as an effective way to reach an optimum combination of strength and durability. Ozyildirim (1994) found that when silica fume is added in small amounts (3\% to $5 \%$ ) to concrete with up to $47 \%$ slag at a water-cement ratio from 0.40 to 0.45 , economical concrete with adequate strength (55 MPa or 7975 psi) can be produced. The New York State Department of Transportation (Streeter, 1996) began using HPC in 1994 to produce long-lasting and more durable bridge decks. The concrete contained 20\% Class F fly ash and $6 \%$ microsilica or silica fume with a total cementitious content of $405 \mathrm{~kg} / \mathrm{m}^{3}$ (682 $\mathrm{lb} / \mathrm{yd}^{3}$ ) and a low w/cm, while maintaining the workability by using WRA. There are other evidences (not referred here for brevity) that combination of silica fume and slag or fly ash can be successfully used to produce HPC for bridge decks.

4. From Figure 4.1 (a), (b), and (c), it is found that with the same aggregate (L1), the compressive strength increased for all the ages, as w/cm ratio was reduced. For reduction of w/cm from 0.40 to 0.35 , the 28 -day strength increased by $13 \%$, and for reduction of w/cm from 0.35 to 0.3 , the strength increased by $16 \%$. 


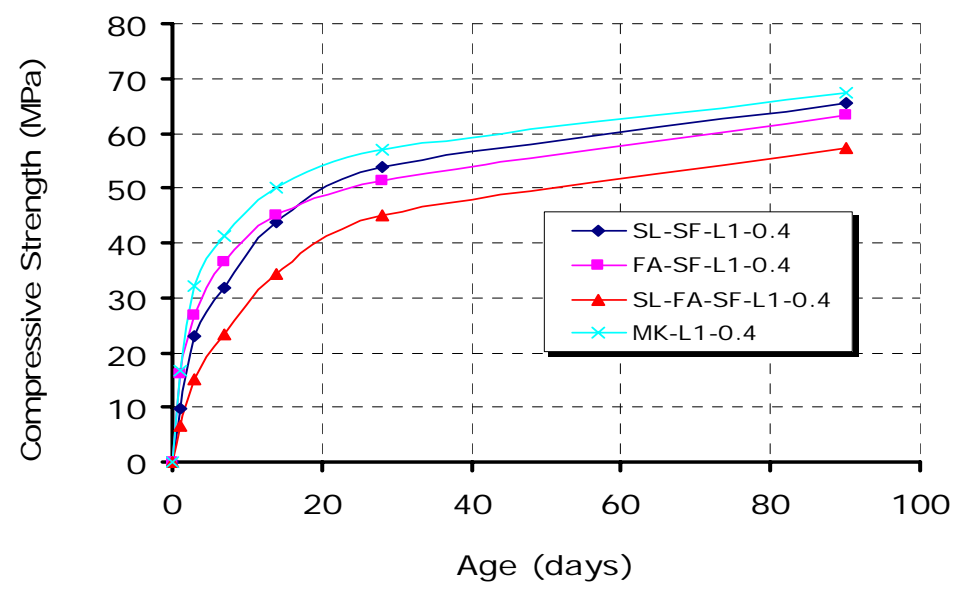

(a)

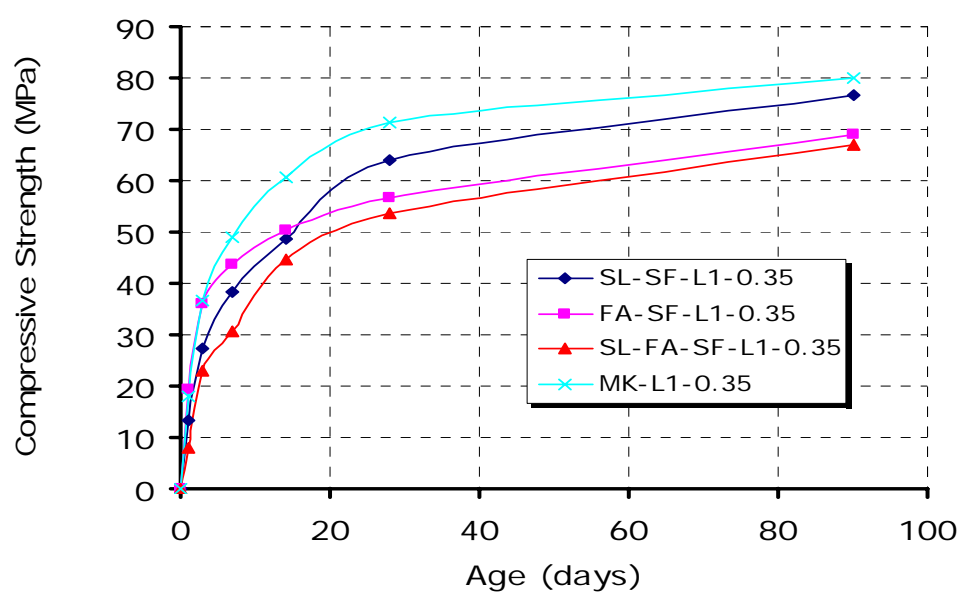

(b)

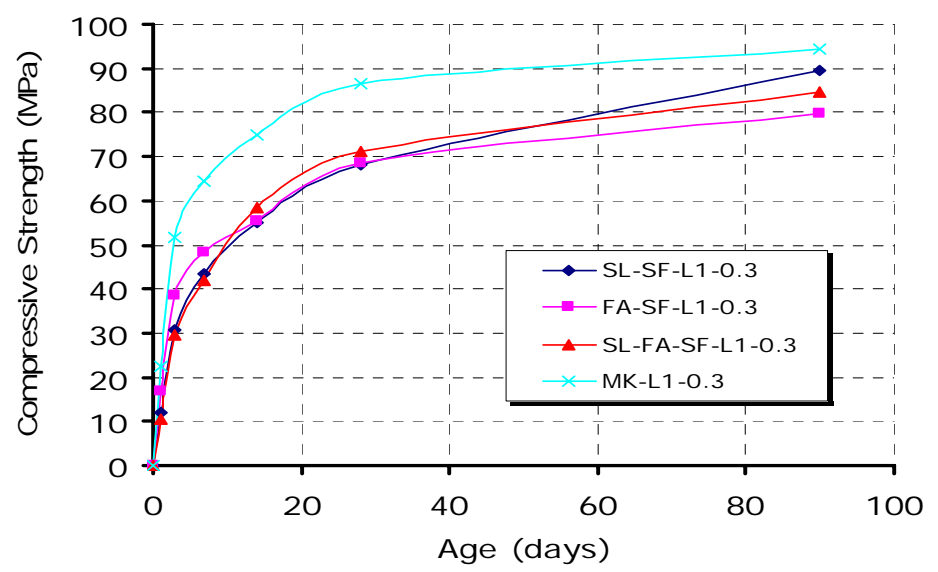

(c) 


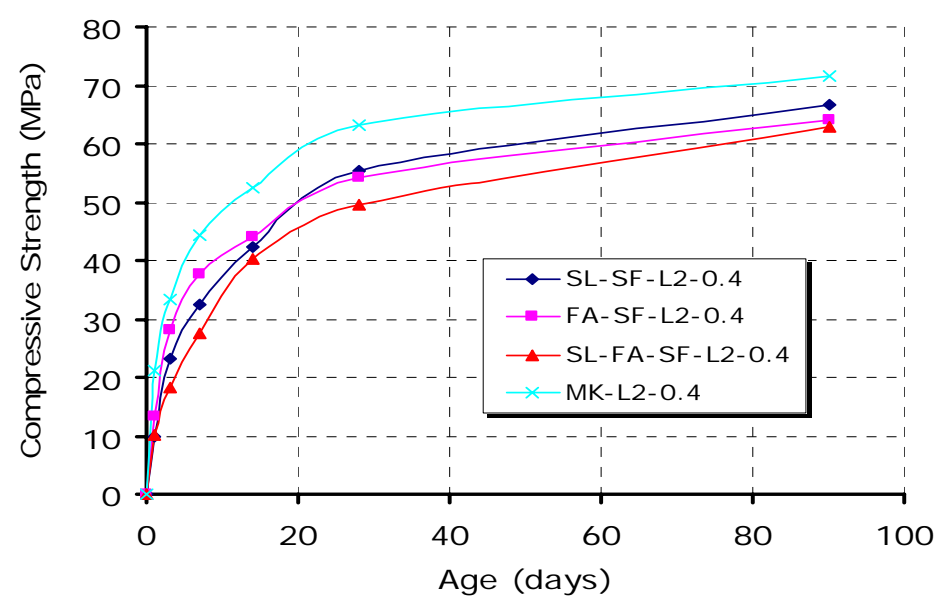

(d)

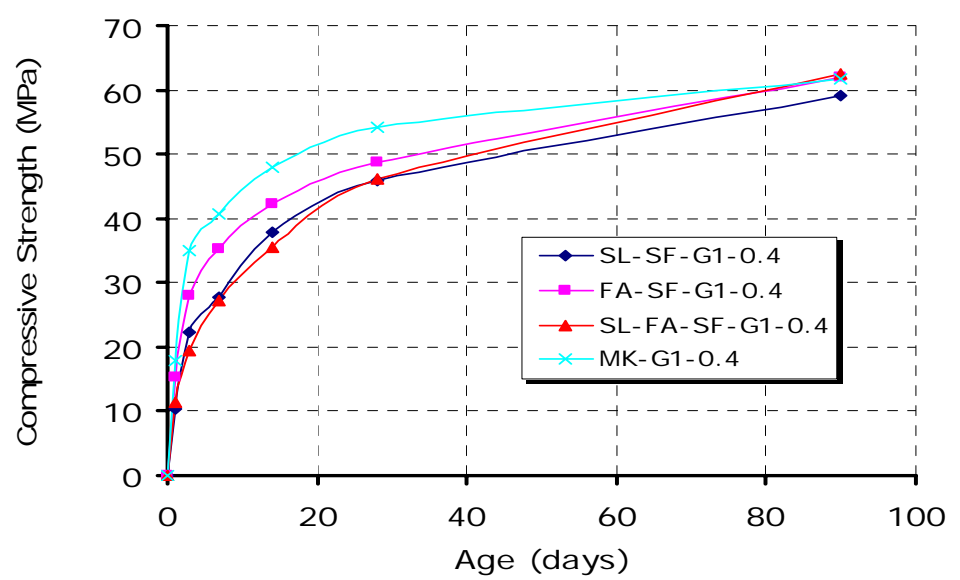

(e)

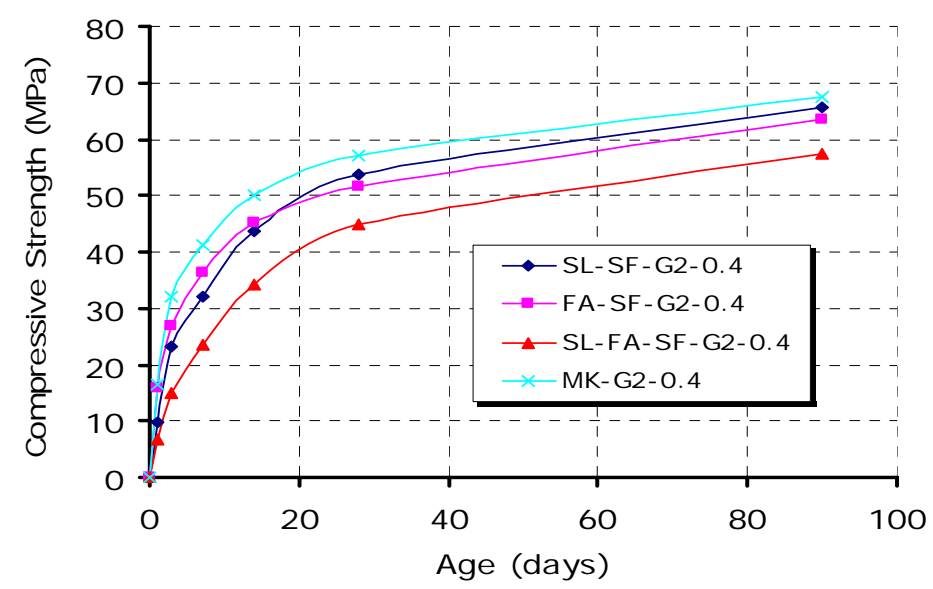

(f)

Figure 4.1 Compressive strength with time up to 90-day for 24 HPC mixtures

$$
(1 \mathrm{MPa}=145 \mathrm{psi})
$$


Figure 4.2 shows that limestone HPC had 10 to 15\% more 28-day compressive strength (average) than gravel HPC at all replacement levels. Since different types of SCM were used in this study, it is difficult to draw any conclusion on the effect of SCM percentage on strength or modulus of elasticity. However an interesting trend is observed for all types of aggregates that with the increase in SCM content from 10 to $45 \%$, there was a significant drop in compressive strength, except for a slight rise from 25\% (FA + $\mathrm{SF})$ to $35 \%(\mathrm{SL}+\mathrm{SF})$. This may be due to the use of slag which is hydraulic in nature and possibly formed more strength forming gel at 28-day in spite of being used at a higher quantity than fly ash.

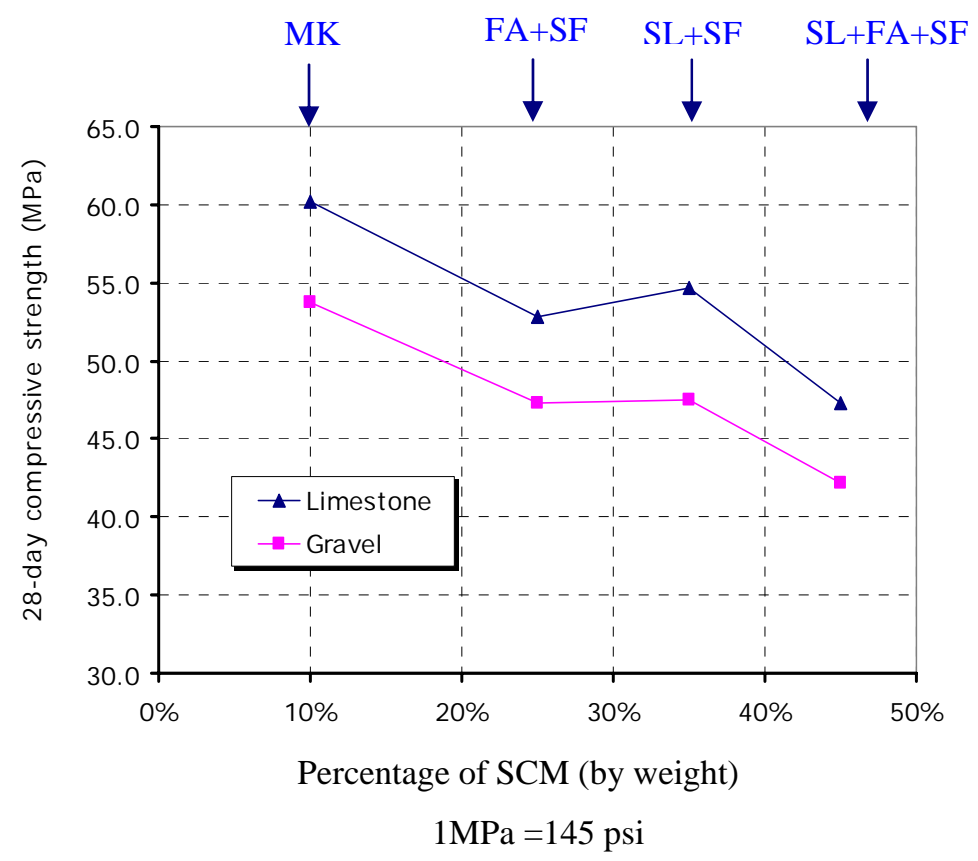

Figure 4.2 Effect of SCM on 28-day compressive strength

In order to validate the laboratory data from current research, the field data were collected with the help of the WVDOH personnel and ready mix plants of the state of WV. Field data were collected from different bridge projects built during the last three 
years. A comparative plot of compressive strength of the present study and field data from various projects complete statewide is shown in Figure 4.3. The laboratory results fitted very well within the range of concrete produced by ready mix plants. Since no data is available for 90-day, no comparison could be made for this age, however the increasing trend is observed as expected.

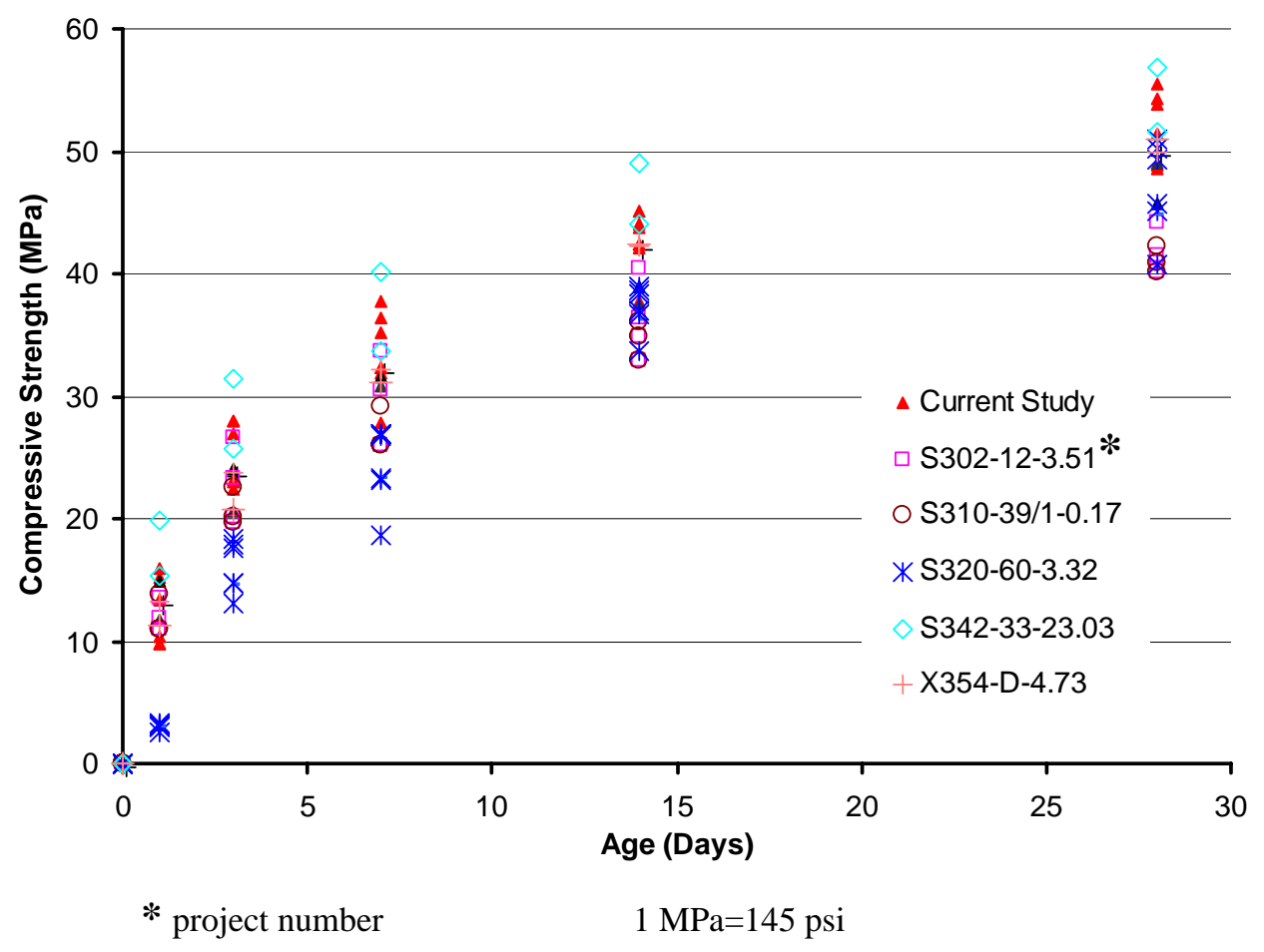

Figure 4.3 Compressive strength from current study compared to ready mix plants in WV 


\subsubsection{Modulus of elasticity and Poisson's ratio}

The Poisson's ratio for all mixtures ranged from 0.19 to 0.24 , as listed in Appendix A. The mixtures with limestone L1 had slightly higher values than those of other mixtures.

Figure 4.4 shows the modulus of elasticity of mixtures at 28 days as function of percentage of SCM for two coarse aggregate types. It was found that with the increase in SCM content from 10 to $45 \%$, the modulus of elasticity changed in a similar manner to compressive strength (Figure 4.2). The modulus of elasticity of HPC mixtures with limestone was greater than for corresponding mixtures with gravel, for all SCM types and percentages. This shows the clear effects of aggregates on stiffness of concrete. Limestone being more angular in shape had a better inter-aggregate locking, which underwent less deformation for a given stress. This also corroborates well with the dynamic modulus values for both of these aggregates as reported by Fan (2005).

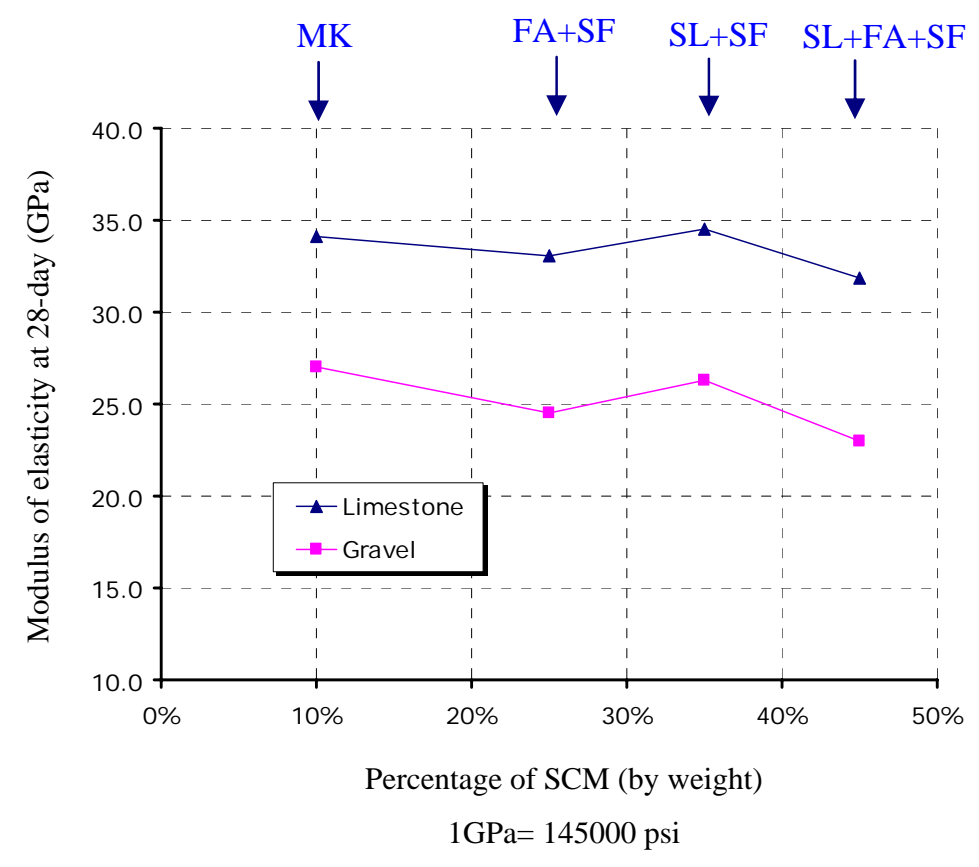

Figure 4.4 Effect of SCM on 28-day modulus of elasticity 
The experimental data of 28-day compressive strength and modulus of elasticity were compared with existing ACI 318 code (2001) equation and ACI 363 recommendation (1997). It was found that ACI 318 code overestimated the values; while ACI 363 predictions were closer to the experimental data, though ACI 363 slightly underestimated HPC with limestone and overestimated HPC with gravel.

A modified expression based on the WV sources of aggregates, which may be considered as similar to many such limestone and gravel sources in this region, is proposed as follows. A separate expression was developed for each aggregate type (limestone or gravel) using experimental data from two batches, two different types of limestone or gravel and all SCM combinations. The slope of ACI 363 expression was kept unaltered, and the data was plotted with a best linear fit (Figure 4.5) to obtain a new constant on the right hand side of the equations.

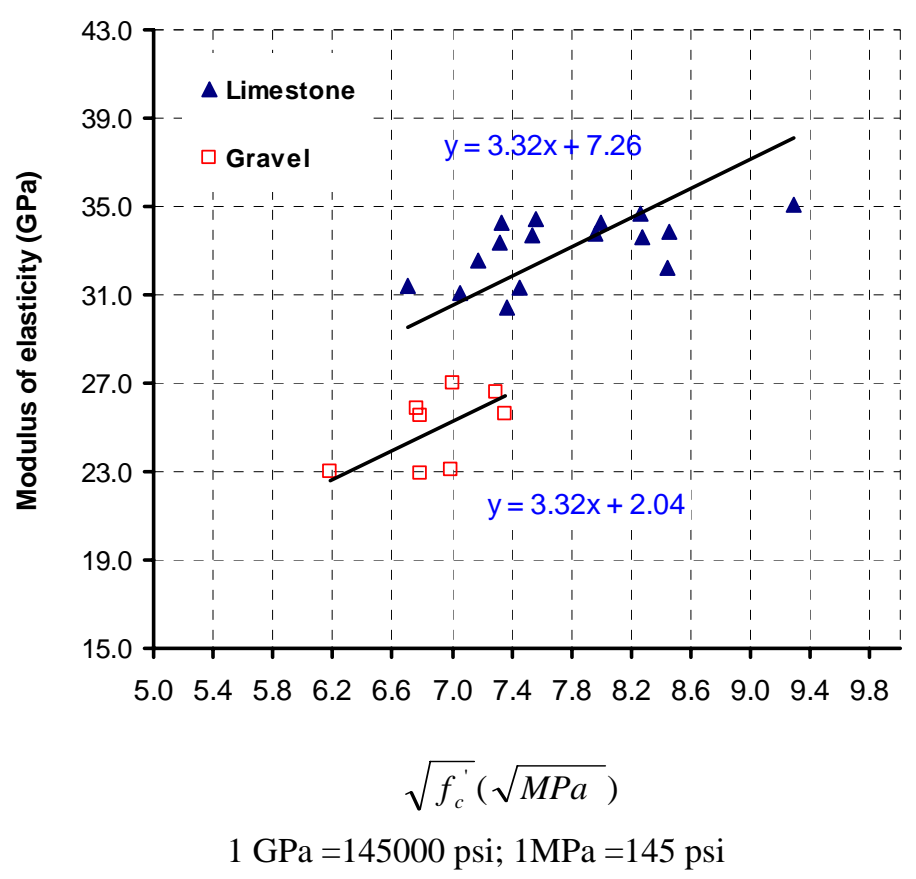

Figure 4.5 Relationships between 28-day modulus of elasticity and compressive strength 
ACI 363 expression for strength of 24 to $83 \mathrm{MPa}$ is as follows:

$$
E_{c}=3.32\left(\sqrt{f^{\prime}}\right)+6.9
$$

where $E_{c}$ is the modulus of elasticity expressed in GPa and $f^{\prime}{ }_{c}$ is the compressive strength expressed in MPa. Proposed modified expressions for strength of 40 to $65 \mathrm{MPa}$ in this study are as follows:

$$
\begin{array}{ll}
E_{c}=3.32\left(\sqrt{f^{\prime}}\right)+7.26 & \text { for limestone } \\
E_{c}=3.32\left(\sqrt{f^{\prime}}\right)+2.04 & \text { for gravel }
\end{array}
$$

The notations have the same meaning as in Eq. (4.1). The $\mathrm{R}^{2}$ for Eq. (4.2) and Eq. (4.3) are 0.899 and 0.864 respectively.

The chloride permeability tests were conducted simultaneously during this research by other members (Fan, 2005). The results showed that, for the mixtures with SCM combination of SL-FA-SF, the chloride permeability values were much higher than the requirement by WVDOH specification (WVDOH, 2003), even for the w/cm as low as 0.35. Based on this consideration, all the mixtures with SL-FA-SF were eliminated from further study.

\subsection{Direct Tensile Strength and Modulus of Elasticity in Tension}

\subsubsection{Significance of the test}

Due to several technical difficulties, very limited direct tensile strength test results have been reported in the literature, especially for HPC with different SCM combinations. However, tensile strength is regarded as an important parameter for evaluating the occurrence of cracks in concrete structures. A reliable and repeatable 
direct tensile test is essential for the better understanding of the cracking properties of HPC mixtures.

\subsubsection{Results and discussions}

\subsubsection{Validation of the testing system}

In Chapter Three, the direct tensile testing procedure was discussed. In order to evaluate the feasibility of the overall testing procedure, several trial tests were conducted to define the formal tests. Figure 4.6 shows typical stress-strain curves recorded during the trial tests. The readings of the two strain gauges, which were located at the opposite sides of the notched area, were close to each other up to $70 \%$ of the maximum load. This indicated that the current testing method can effectively minimize the eccentricity of the load, which is a well known technical problem in the direct tensile test. However, due to the heterogeneous characteristics of the concrete at high stress level, the development of micro-cracks as well as the development of flaws inside the concrete may differ from one side to the other. Thus, two curves deviate more at higher stress levels. Similar results were found in the work reported by Somsak et al. (2003).

The trial tests further showed that the preparation of the specimen and epoxy bonding of the end steel dishes were also effective. The epoxy had enough strength to transmit the stresses to the concrete until specimens failed at the notch. 


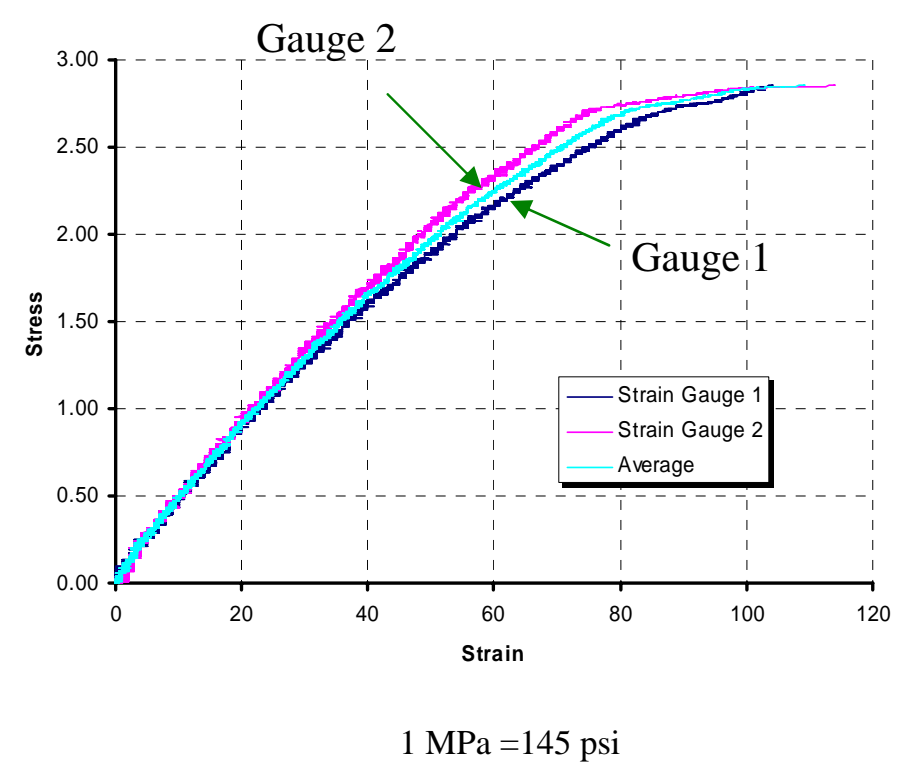

Figure 4.6 Readings from two strain gauges on opposite sides

\subsubsection{Selection of mixtures and failure description}

A total of 16 mixtures were selected from the initial 24 mixtures for this study. The detailed mixture designs are given in Table 3.9 and Table 3.10, from which, the mixtures with SL-FA-SF were excluded. For every mixture, the concrete at ages of 3, 7, 14, 28 and 90-day were tested. For every age, three replicate specimens were tested to assess repeatability and validity. Totally 240 specimens were tested in the tensile test program.

Figures 4.7 (a), (b), (c), and (d) show the failure cross-sectional surfaces of the broken specimens. The specimens in Figures 4.7 (a) and (b) are for the limestone HPC at the ages of 3-day and 28-day respectively. The specimens in Figures 4.7 (c) and (d) are for gravel HPC at the ages of 3-day and 28-day respectively. From those figures it is seen that at the early age (3-day), the cementitious paste carried most of the stress, and in general the coarse aggregates were pulled out directly from the cementitious matrix. However at a later age (28-day), the concrete acted as an integral composite between 
aggregates and matrix, and as a result, most of aggregates split into two at the notch section. This phenomenon was observed for both limestone and gravel.

Figure 4.8 shows side views of typical specimens after failure, with different torturous crack propagation paths for different specimens.

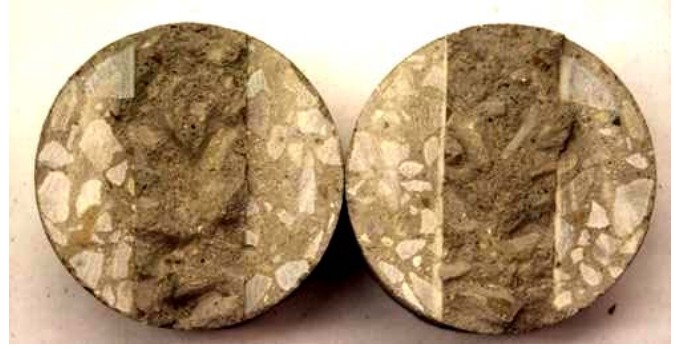

(a)

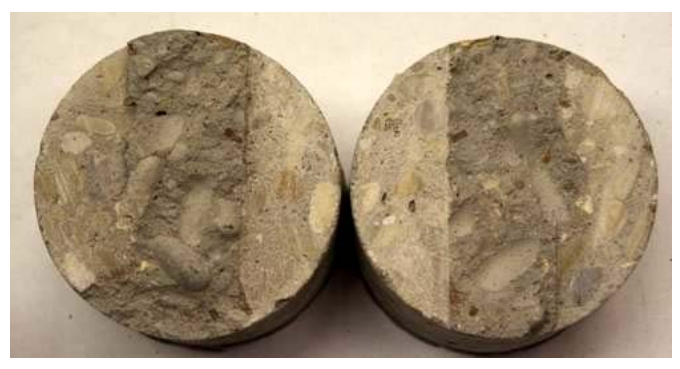

(c)

(a) Limestone at 3-day

(c) Gravel at 3-day

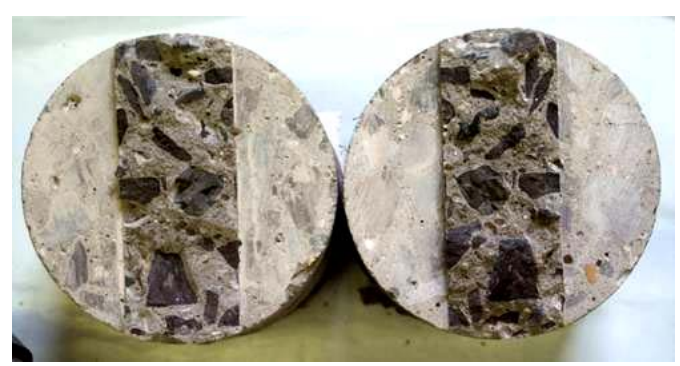

(b)

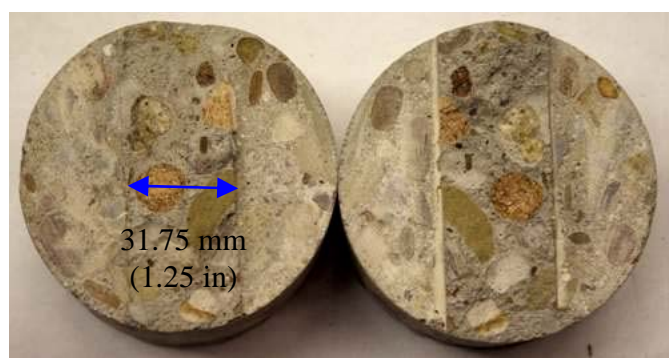

(d)

(b) Limestone at 28-day

(d) Gravel at 28-day

Figure 4.7 Cross-section of broken specimens 


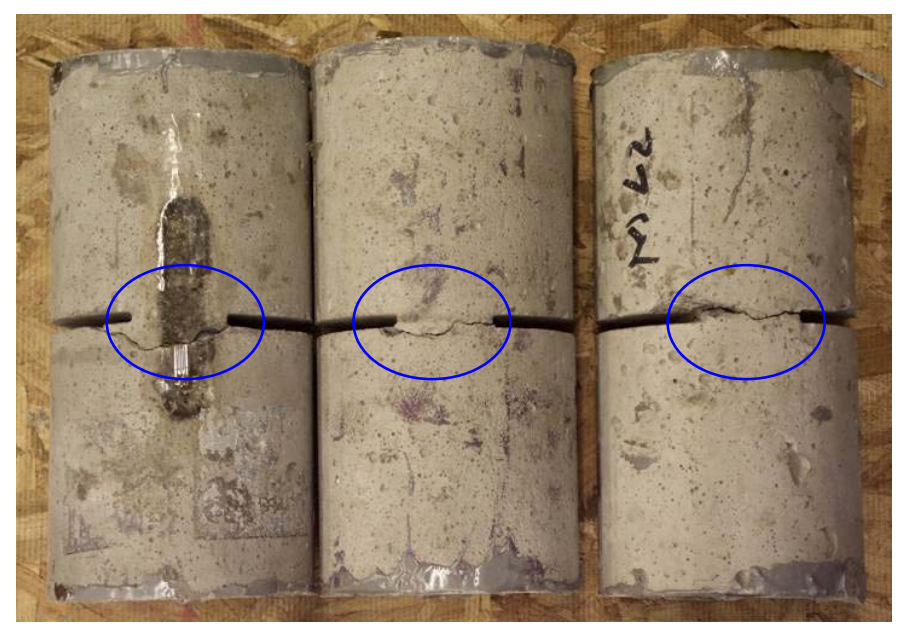

Figure 4.8 Side view of typical broken specimens

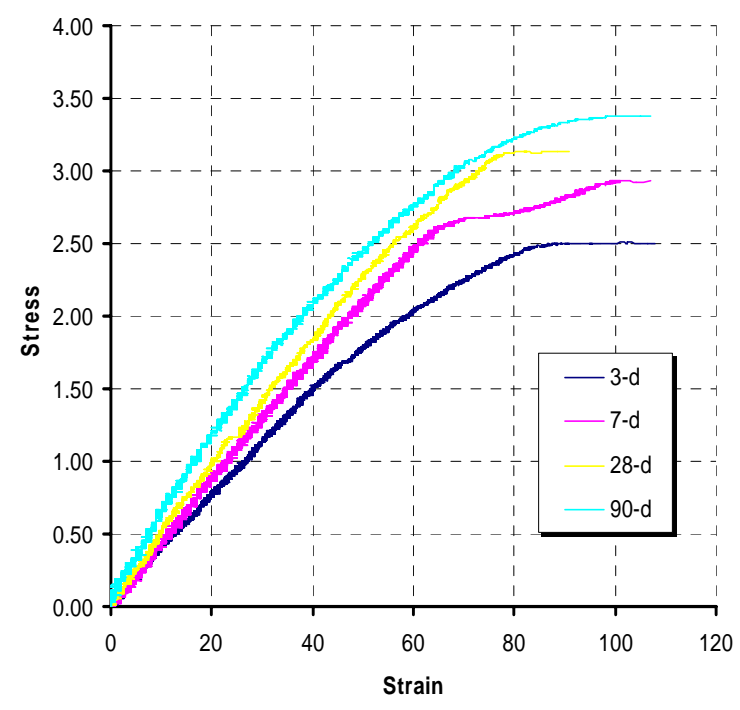

(b) Stress-strain curves for HPC SL-SF-L1-0.3

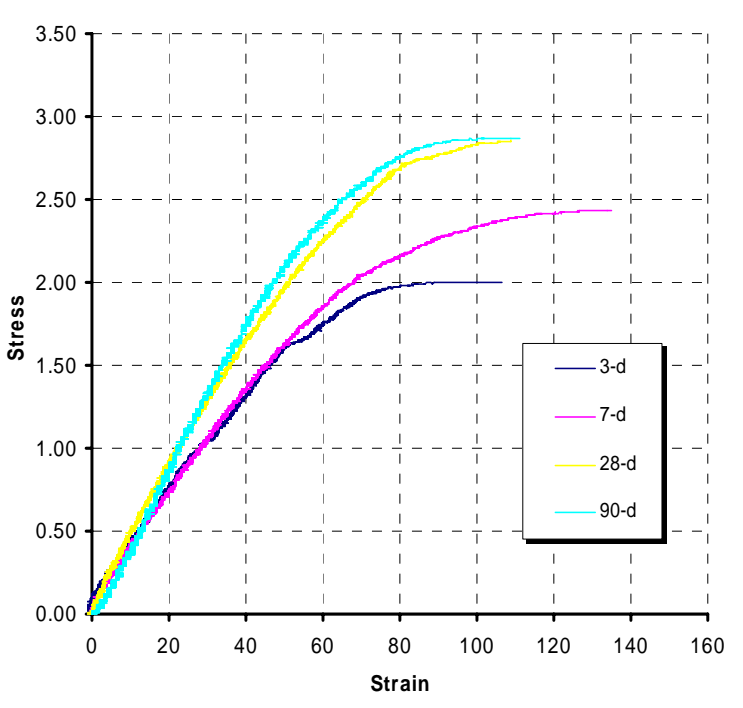

(a) Stress-strain curves for HPC FA-SF-G2-0.4

Figure 4.9 Typical stress-strain curves of HPC in tension 


\subsubsection{Tensile strength}

Figures 4.9 (a) and (b) show typical stress-strain curves recorded in this study for mixture FA-SF-G2-0.4 and SL-SF-L1-0.4, respectively. A good linear relationship was found for a stress up to $30 \%$ of maximum value, and between $30 \%$ to $70 \%$, nonlinearity appeared. After $70 \%$ of maximum stress, the inelastic behavior became prominent.

The tensile strength test results for all 16 HPC mixtures are listed in Appendix B; Figures 4.10 (a), (b) and (c) display the tensile strength development up to 90-day for all HPC tested. The tensile strength data and strength-age relationship reveal the following findings:

1) Tensile strength increased rapidly up to 14-day, and then the increase became gradual. The slower tensile strength development after 14-day compared to compressive strength development was possibly due to the formation of drying shrinkage cracks within specimens as all tensile test specimens were cured in air after 24 hours while specimen for compressive strength were cured under water. The 90day strength was slightly lower than the 28-day strength, especially for HPC with $\mathrm{w} / \mathrm{cm}=0.3$. This phenomenon is referred in the literature as "strength retrogression" (Aitcin, 1998). Most HPC have higher cementitious content and lower w/cm compared to normal concrete. Very often after 28-day, the hydration stops or becomes slower within the concrete due to self-desiccation. Particularly, for small size specimens, the dry condition aggravated the phenomenon. Thus, the drying results in an induced stress gradient at the notched area, and lead to stress concentration. The stress concentration and microcracks affect the tensile strength for the specimens tested at later ages such as 90-day. 
2) The trend for strength development of limestone HPC was slightly different compared to gravel HPC, as shown in Figure 4.10. In general, the limestone HPC achieved higher tensile strength than gravel HPC.

3) At 28-day, HPC with MK had the highest strength followed by SL-SF and FA-SF (Figure 4.11). Also, the difference between SL-SF and FA-SF was in general small.

4) Reduction of w/cm from 0.4 to 0.35 caused much more increase in tensile strength than reduction of w/cm from 0.35 to 0.30 , as shown in Figure 4.12. This illustrates that achieving higher compressive strength by lowering w/cm from 0.4 to 0.35 does not necessarily results in increase of cracking potential since in this case both higher compressive strength and higher tensile strength are achieved, and better tensile strength leads to reduce the cracking tendency. But further decrease of w/cm from 0.35 to 0.30 caused only slight increase in tensile strength with much higher gain in compressive strength, which can increase the risk for cracking. These relationships among cracking, compressive and tensile strengths will be discussed in details in Chapter Five. 


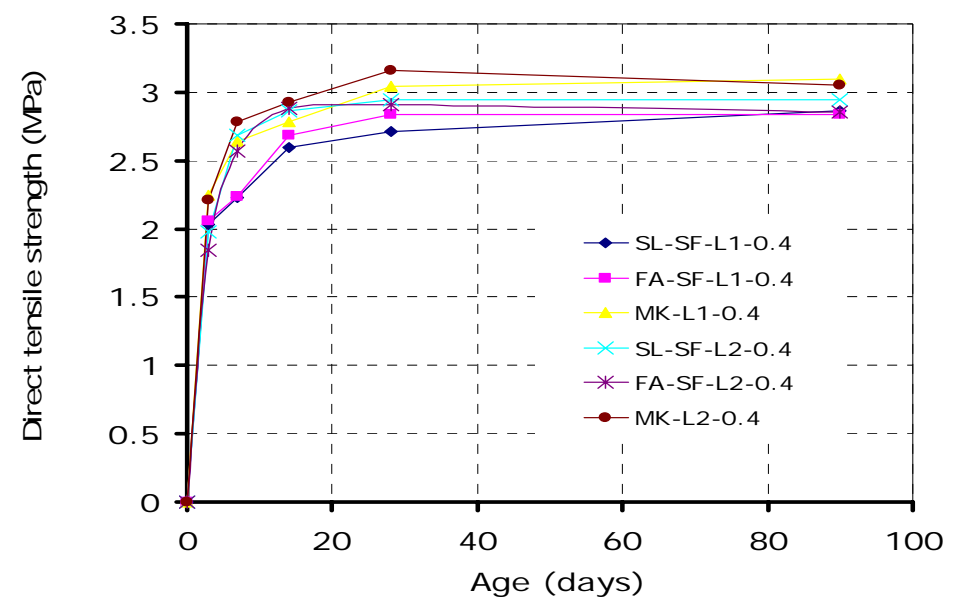

(a)

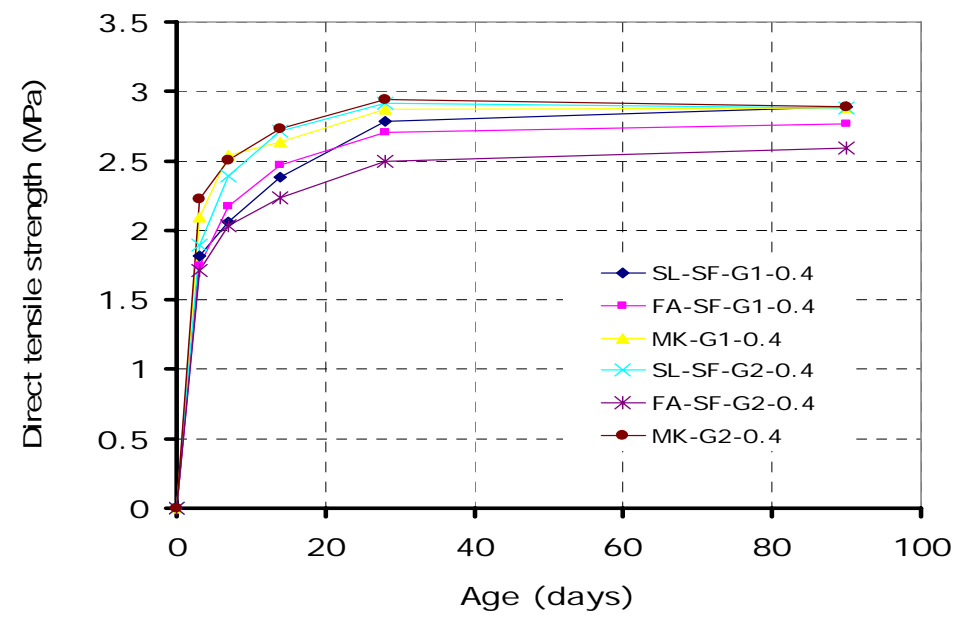

(b)

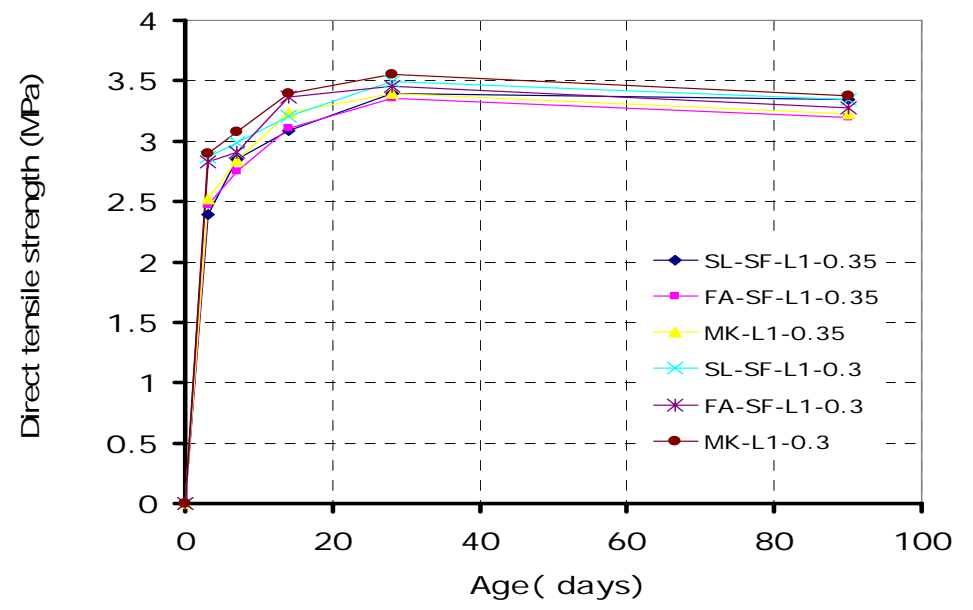

(c)

$1 \mathrm{MPa}=145 \mathrm{psi}$

Figure 4.10 Direct tensile strength with time for up to 90-day 


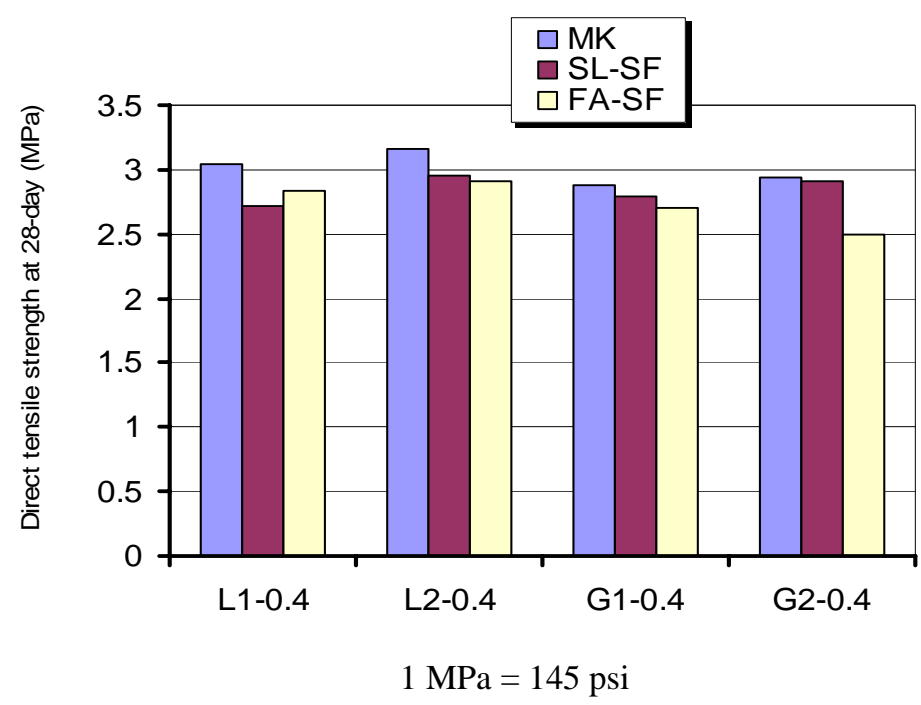

Figure 4.11 Direct tensile strength at 28-day for HPC with different SCM

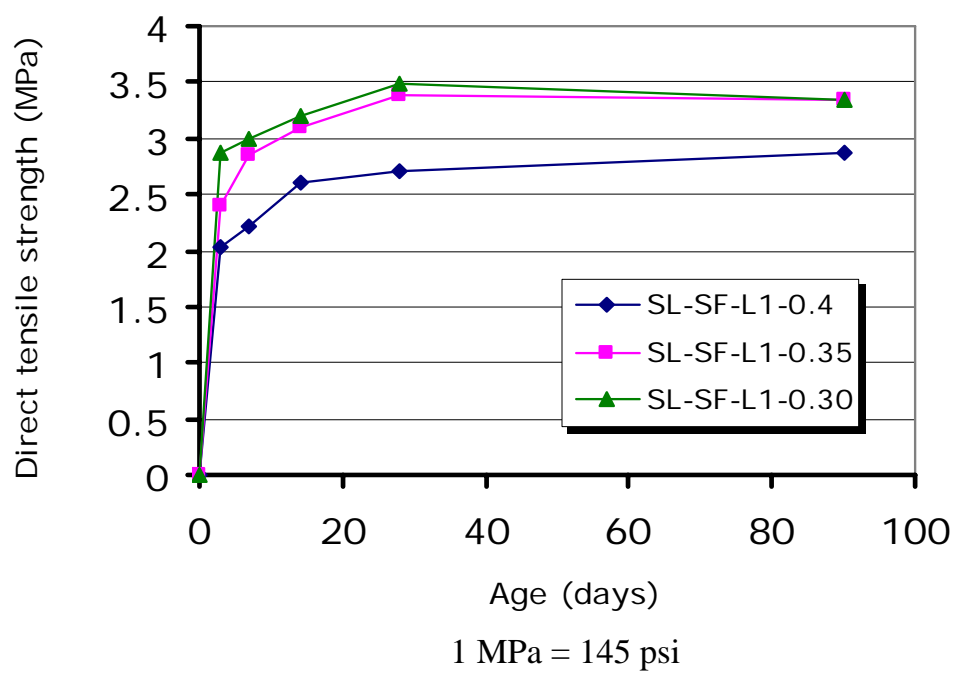

Figure 4.12 Direct tensile strength with time for HPC with different w/cm

\subsubsection{Relationship between direct tensile strength and compressive strength}

The tensile strength is usually measured by an indirect approach such as by modulus of rupture or by splitting tensile strength. The existing predictive equations on the 
relationships between tensile strength and compressive strength are mostly between splitting tensile strength and compressive strength. Some of those relationships are presented as follows:

(a) The ACI 363R (1997) equation:

$$
f_{s p}=0.59\left(f_{c}^{\prime}\right)^{0.5}
$$

where $f_{s p}$ is splitting tensile strength, and $f^{\prime}{ }_{c}$ is specific compressive strength with a range of 21 to $83 \mathrm{MPa}$.

(b) The CEB-FIP (1978) equation:

$$
f_{s p}=0.273\left(f_{c}^{\prime}\right)^{2 / 3}
$$

(c) The proposed equation by Ahmad and Shah (1985)

$$
f_{s p}=0.462\left(f_{c}^{\prime}\right)^{0.55}
$$

for t concretes with $f^{\prime}{ }_{c}$ less than $84 \mathrm{MPa}$.

(d) The CEB-FIP (1990) equation

The CEB-FIP (1990) is the first standard which accepted the direct tensile strength, based on RILEM CPC 7, as the design parameter. CEB-FIP (1990) also permits the use of the following predictive equation for tensile strengths in the absence of accurate data:

$$
f_{t}=f_{t o}\left(\frac{f_{c}^{\prime}}{10}\right)^{2 / 3}
$$

where $f_{t}$ is direct tensile strength in $\mathrm{MPa} ; f_{\text {to } \min }=0.95 \mathrm{MPa}$ and $f_{\text {to } \max }=1.85 \mathrm{MPa}$.

(e) The ACI $209 \mathrm{R}$ (1992) equation:

$$
f_{t}=0.0069\left(w f_{c}^{\prime}\right)^{1 / 2}
$$


Where $w$ is the unit weight of concrete, which if assumed as $w=2300 \mathrm{Kg} / \mathrm{m}^{3}$, then Eq.

(4.8) can be rewritten as:

$$
f_{t}=0.331\left(f_{c}^{\prime}\right)^{1 / 2}
$$

(f) The proposed equation by Zhang et al. (2001):

$$
f_{t}=0.47\left(f_{c}^{\prime}\right)^{1 / 2}
$$

The relationship between direct tensile strength at different ages and square root of compressive strength $\left(f_{c}^{\prime}\right)^{1 / 2}$ from the present study is plotted in the Figure 4.13. The prediction equations by ACI Committee 363R, ACI 209-R, and Zhang et al. (2001) are also included in Figure 4.13. The graph clearly shows that at higher compressive strength level, the tensile strength does not increase proportionately with the increase in $\left(f_{c}^{\prime}\right)^{1 / 2}$.

The best fit equation as obtained by regression analysis is:

$$
f_{t}=0.395 f_{c}^{10.5}
$$

The correlation coefficient: $R^{2}=0.86$.

From Figure 4.13, it is observed that ACI 363R equation (Eq. 4.4) has a much higher tensile strength prediction than the data obtained from this study. This is expected as the splitting strength values for a given concrete are always greater than direct tensile strength values, and the ACI 363R relationship is based on splitting tensile strength. The ACI 363R is provided for comparison considering its wide application. The prediction by Zhang et al.(2001) (Eq. 4.10) gives an upper bound for the testing data set, while ACI 209R equation (Eq. 4.9) gives a lower bound solution for the testing data set. 


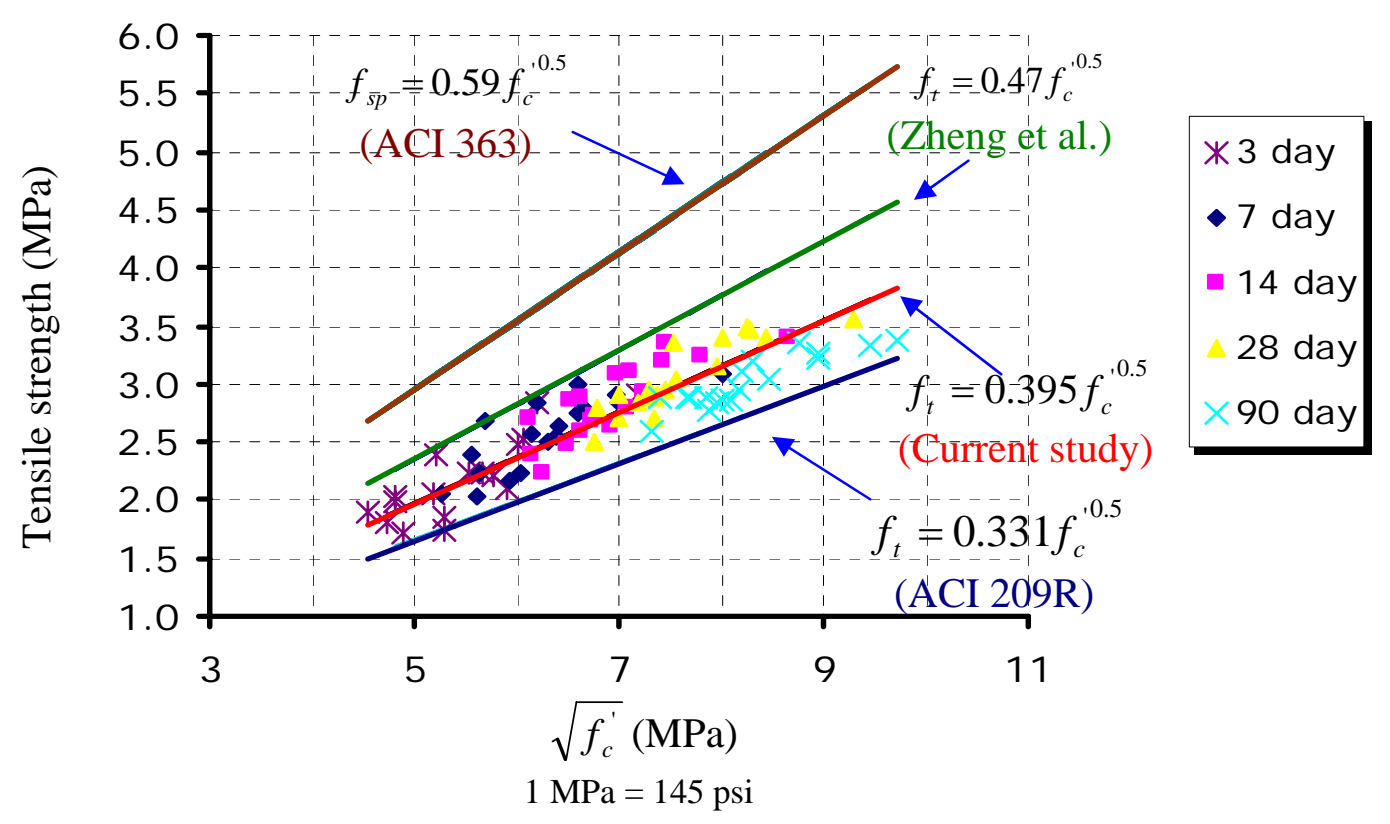

Figure 4.13 Relationship between direct tensile strength and $\left(f_{c}^{\prime}\right)^{1 / 2}$

\subsubsection{Tensile strain}

Figure 4.14 shows the average maximum strain at failure load for different ages. The tensile strain capacity does not vary significantly with aggregate type, w/cm or curing ages. All the maximum strains in this study were within the range from $90 \mu \varepsilon$ to $120 \mu \varepsilon$.

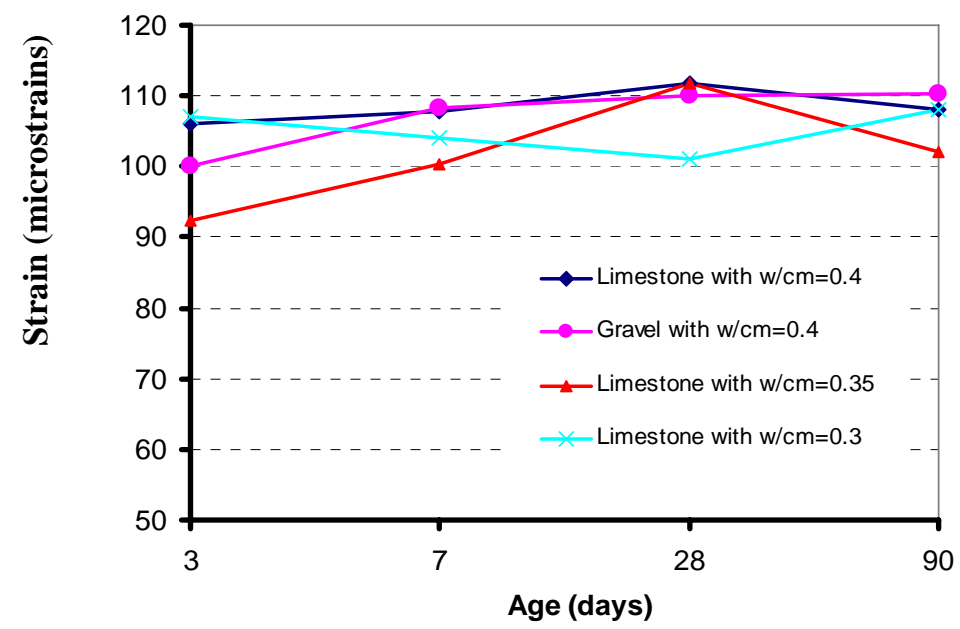

Figure 4.14 Maximum tensile strains of different HPC mixtures 


\subsubsection{Tensile modulus of elasticity}

The tensile stress-strain curve is similar in shape to that in compression. However due to the presence of cracks, the non-linearity behavior in tensile stress-strain curve occurred at lower stress/strength ratio than in compression. In this study, the method used to calculate modulus of elasticity in tension is based on ASTM C469 with certain modifications as follows:

1. In ASTM C469, the static or chord modulus of elasticity is determined by two points: the first point is where the strain is equal to 1/40th of the maximum compressive strain of concrete, which is approximately $50 \mu \varepsilon$. The second point is where the load reaches $40 \%$ of maximum load. As was mentioned previously, the maximum tensile strains measured in this study were between $90 \sim 120 \mu \varepsilon$, accordingly, for tensile modulus of elasticity, the first point P1 was selected for a strain equal to $3 \mu \varepsilon$ by following the same ratio used in ASTM C469 (Figure 4.15).

2. The tensile modulus of elasticity was used to analyze the cracking of the ring specimen. Since the cracking occurred at a high stress level, it is logical to determine the tensile modulus of elasticity taking into account a point at a high stress level. The second point P2 was therefore selected as the value corresponding to maximum stress as shown in Figure 4.15 


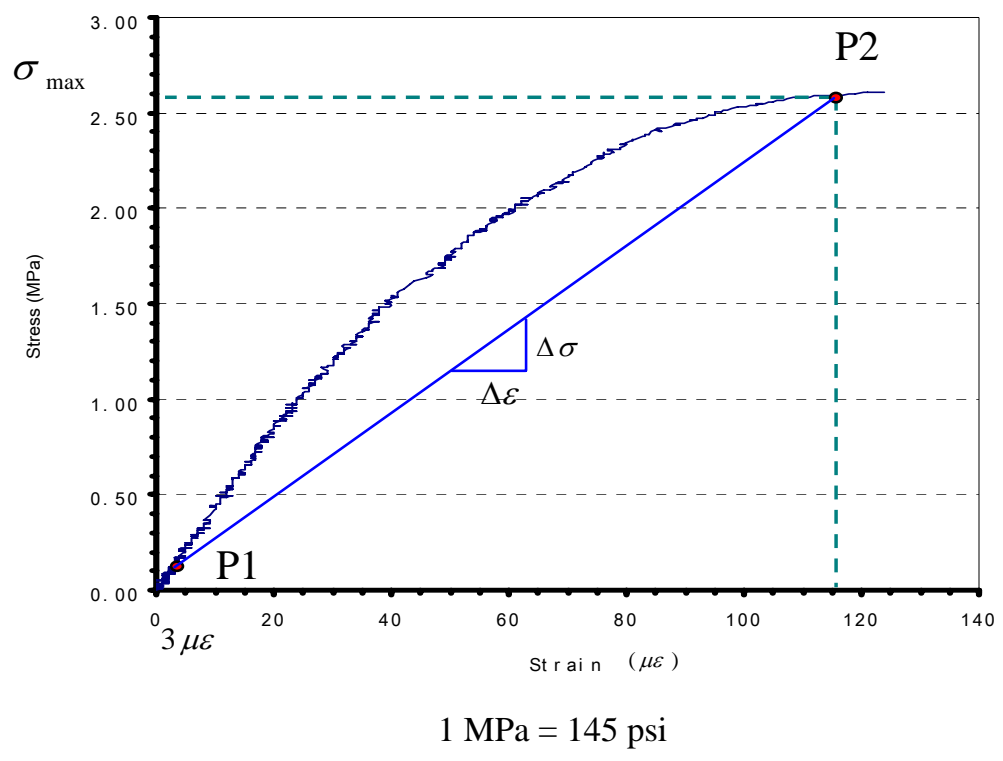

Figure 4.15 Method to calculate the tensile modulus of elasticity

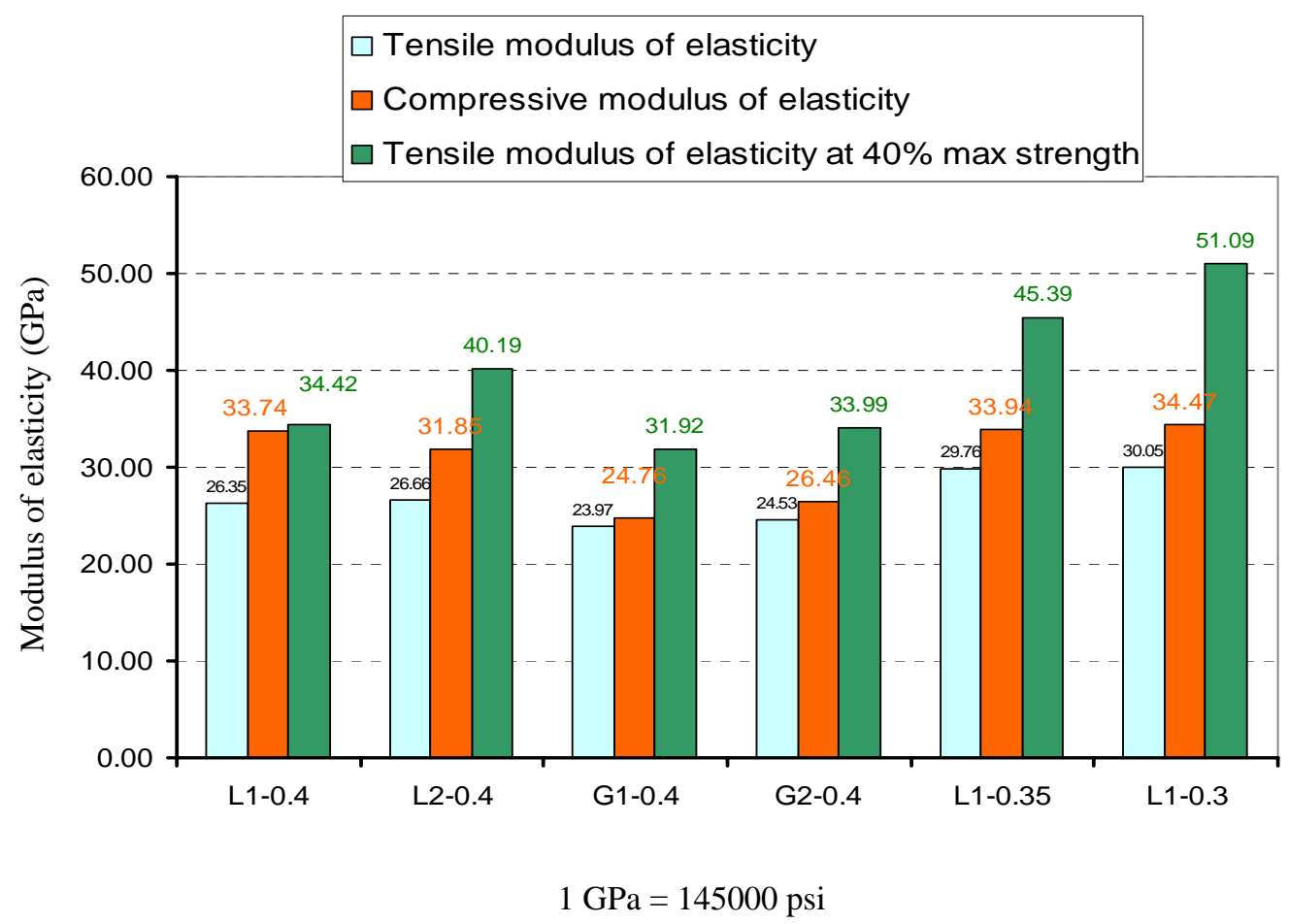

Figure 4.16 Tensile and compressive moduli of elasticity of various HPC mixtures 
Based on the concept described in Section 4.4.2.6, the tensile modulus of elasticity was determined for different HPC. A comparative plot of tensile modulus of elasticity and compressive modulus of elasticity of different mixture groups at 28-day is presented in Figure 4.16. The modulus data presented here for every mixture group is the average of three different mixtures. For example, the L1-0.4 data in Figure 4.16 is the average of SL-SF-L1-0.4, FA-SF-L1-0.4, and MK-L1-0.4. It is found that the tensile modulus of elasticity determined by present proposed method is lower than the compressive modulus of elasticity in all cases. But if the tensile modulus of elasticity was determined with second the point corresponding to $40 \%$ of maximum tensile strength (similar to compressive modulus of elasticity), the values are greater than the compressive modulus of elasticity (as shown in Figure 4.16). However, for the purpose of application of tensile modulus of elasticity in cracking analysis, the second point P2 was taken corresponding to the maximum stress as mentioned before. Figure 4.16 also shows the influence of w/cm on tension modulus of elasticity.

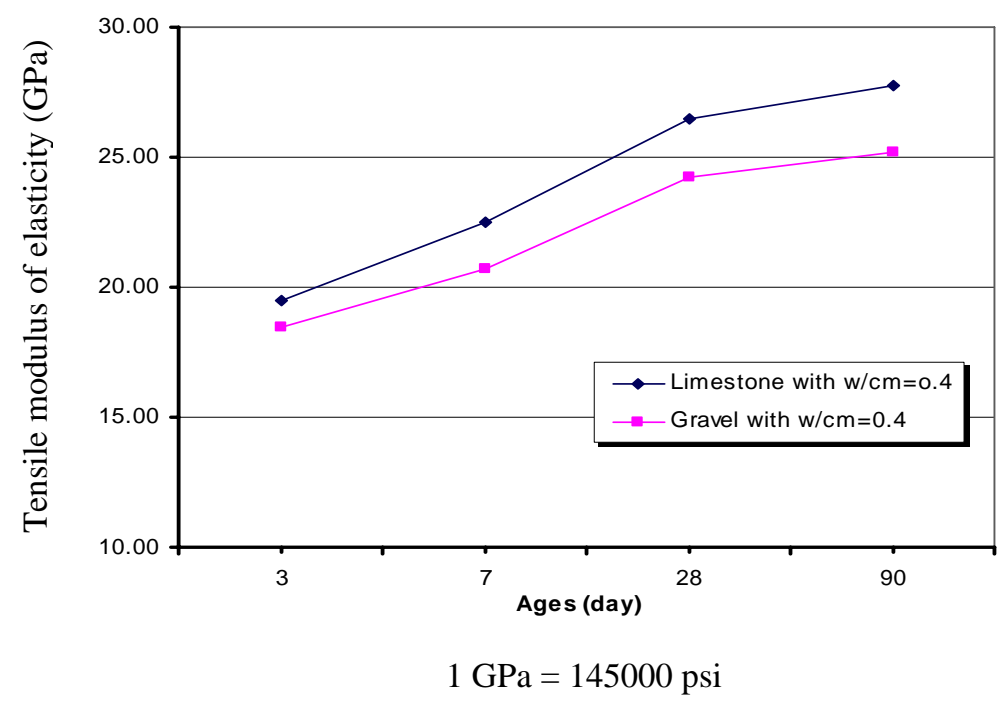

Figure 4.17 Effect of coarse aggregates on tensile modulus of elasticity 
Figure 4.17 shows the tensile modulus of elasticity of HPC with different aggregates and at different ages. Similar to the compressive modulus of elasticity, the tensile modulus of elasticity of HPC with gravel was found to be lower than that with limestone at all ages. The difference increased with the increase in age.

The relationships of the tensile strength and tensile modulus of elasticity are plotted in Figures 4.18 (a) and (b). Figure 4.18(a) is for HPC with limestone and Figure 4.17(b) is for HPC with gravel. A linear relationship between tensile strength and tensile modulus of elasticity for each types of HPC was defined and the corresponding prediction equations based on regression analysis are:

$$
\begin{array}{ll}
E_{t}=8.11 f_{t}+2.99 & \text { for HPC with limestone } \\
E_{t}=6.92 f_{t}+5.20 & \text { for HPC with gravel }
\end{array}
$$

where: $E_{t}$ (in GPa) is modulus of elasticity in tension at high stress level; $f_{t}$ (in MPa) is the tensile strength.

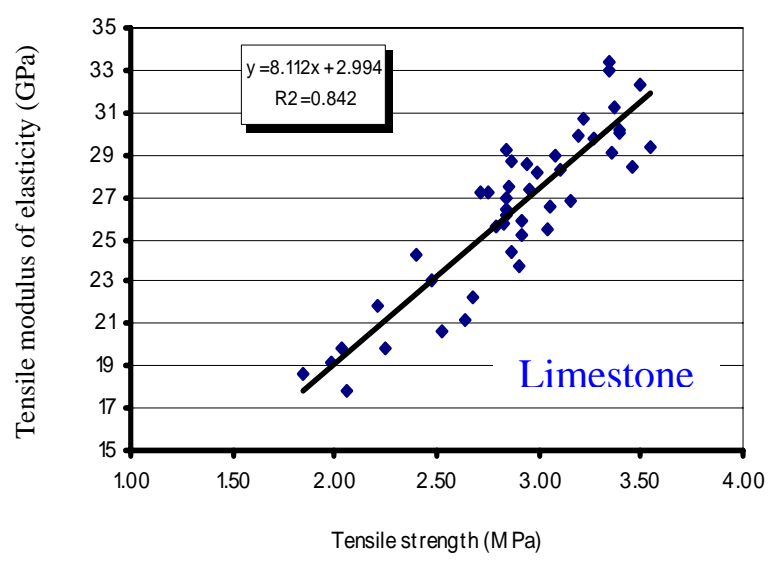

(a)

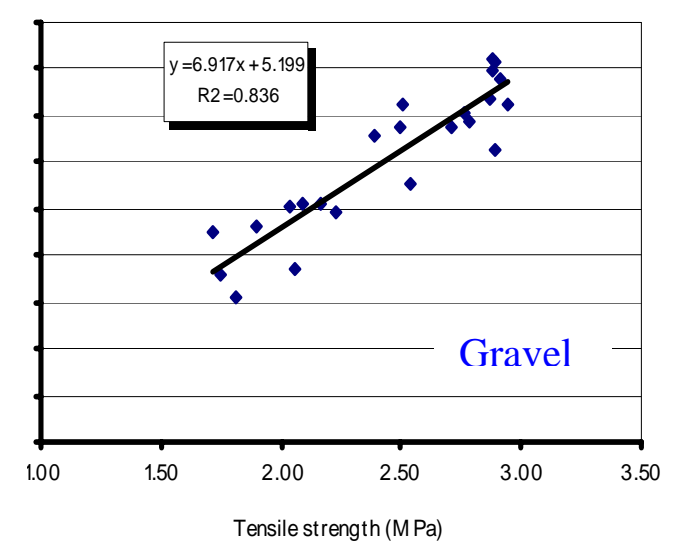

(b)

$$
1 \mathrm{GPa}=145000 \mathrm{psi} ; 1 \mathrm{MPa}=145 \mathrm{psi}
$$

Figure 4.18 Relationships between tensile strength and tensile modulus of elasticity 


\subsection{Free Shrinkage}

\subsubsection{Significance of the test}

As it is discussed in Chapter Two, most bridges experience early cracks due to the restrained shrinkage of concrete. The shrinkage behavior is the single most important issue influencing bridge deck cracking. A free shrinkage test is therefore conducted as quality control of concrete mixtures for bridge applications. The current WVDOH specification also requires the contractor to perform and report the results of free shrinkage tests.

The shrinkage at early age is extremely important for HPC as lower porosity and high volumes of cementitious paste induce higher autogenous shrinkage due to selfdesiccation inside concrete, in spite of spraying water from outside during curing. The autogenous shrinkage sometimes constitutes more than $50 \%$ of total drying shrinkage. In this study, the free shrinkage test measurement started as soon as the specimens were demolded after 24 hours curing. The term "free shrinkage” was used to describe the test, because the data measured in this study includes the sum of autogenous shrinkage caused by self-desiccation and drying shrinkage due to external drying up to 90 days.

\subsubsection{Results and discussions}

The same group of HPC mixtures used for direct tensile test was selected for free shrinkage test. The mix designs are shown in Table 3.9 and Table 3.10. All free shrinkage data of these16 different HPC mixtures are listed in Appendix C.

\subsubsection{Effect of coarse aggregates}

Figure 4.19 shows free shrinkage vs. time curves for HPC mixtures with different coarse aggregates: L1 and G1. No significant difference was found between limestone 
HPC and gravel HPC. But generally, gravel HPC had slightly lower shrinkage compared to limestone HPC.

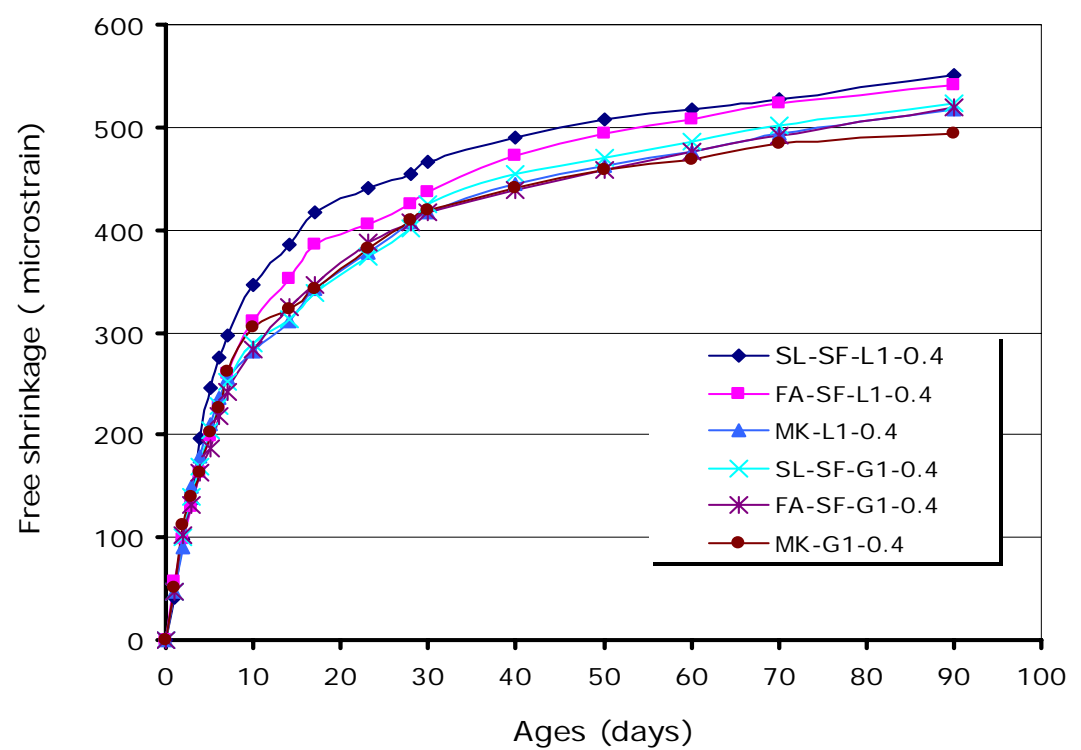

Figure 4.19 Effect of coarse aggregates on free shrinkage of HPC

\subsubsection{Effect of SCM}

Figure 4.20 shows free shrinkage of different HPC mixtures with same w/cm=0.4 and coarse aggregate L1, but different SCM combinations. It is observed that HPC with SCM had a lower free shrinkage up to 90 days compared to control concrete (with the same cementitious material content), except for the mixture with $5 \%$ SF (silica fume). Data for control concrete and HPC with 5\% SF were collected from parallel study conducted by Chatterjee (2004). HPC prepared with solely silica fume had a higher early age shrinkage compared with HPC prepared with other SCM. The binary use of slag + silica fume or fly ash + silica fume significantly reduced the early age free shrinkage. 
Metakaolin showed the best performance in terms of free shrinkage. From Figure 4.20, it is observed that shrinkage of HPC with metakaolin was always lowest among the same mixture group. Similar results were reported by Brook and Megat (2001). In their investigation, it was found that due to increase in the quantity of $\mathrm{MK}$, the autogenous shrinkage would increase as well. However the total free shrinkage was much lower compared to the control concrete.

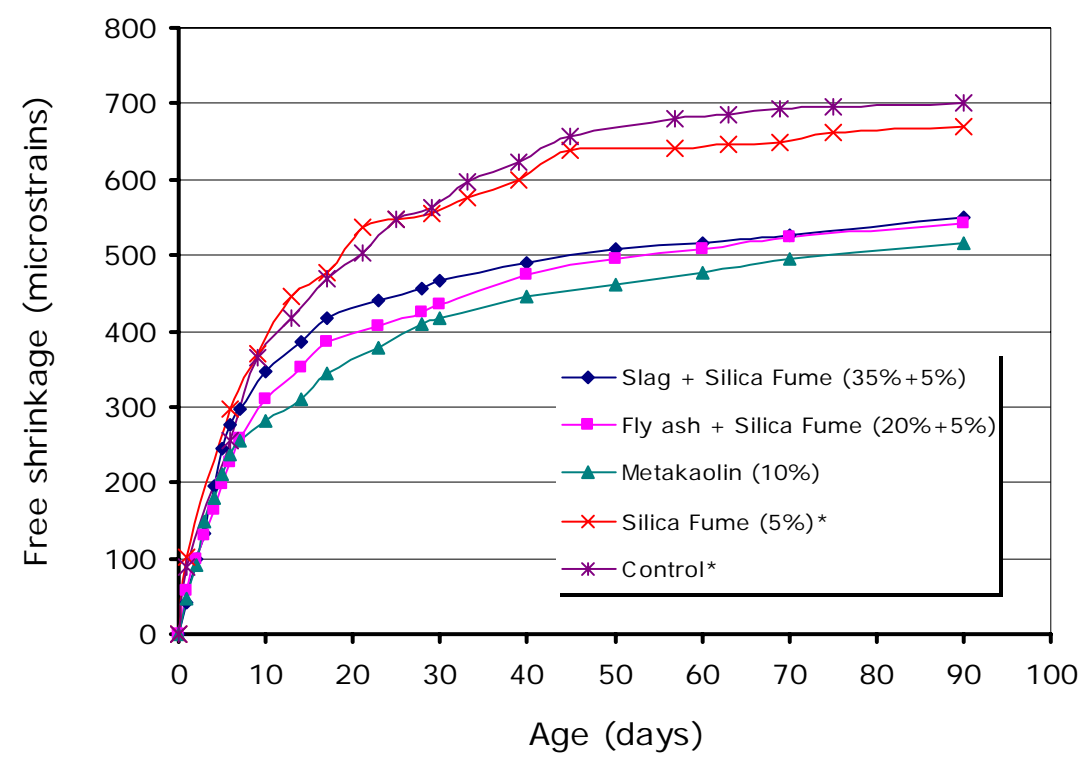

* From a supplementary research conducted by Chatterjee (2004).

Figure 4.20 Effect of SCM on free shrinkage of HPC

\subsubsection{Effect of $w / c m$}

Figure 4.21 shows free shrinkage curves up to 90-day for HPC with different w/cm $(0.4,0.35$ and 0.3$)$. The results show that the lower the $\mathrm{w} / \mathrm{cm}$ ratio is, the higher the free shrinkage is at all ages.

The drying shrinkage component is believed to be related to the total water content of the mixtures (Chatterjee, 2004). Table 4.1 shows the total cementitious material and 
water usage per cubic meter for the three different w/cm ratios. It is found that the water usage for all three w/cm was almost the same, and therefore, drying shrinkage should be approximately at the same level. The observed differences in total free shrinkage are primarily due to self-desiccation or autogenous shrinkage which has a dominant role in free shrinkage properties of the HPC in this study. These HPC (the concrete with lower w/cm) had more cementitious paste (sum of cementitious material and water). These higher cementitious paste volumes might have caused higher shrinkage. The autogenous shrinkage as described above would have been aggravated at higher paste volumes due to the self-desiccation and lower porosity.

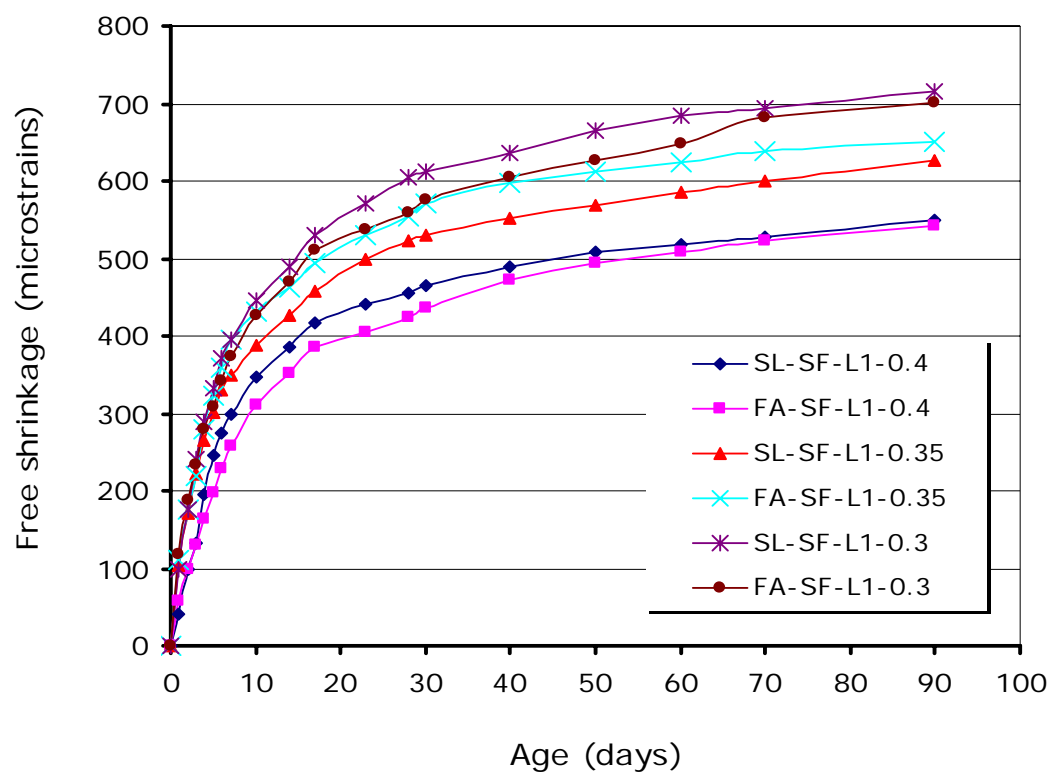

Figure 4.21 Effect of w/cm ratios on free shrinkage of HPC

Table 4.1 Total cementitious material and water usage per $\mathrm{m}^{3}$ of different $\mathrm{w} / \mathrm{cm}$ ratios

\begin{tabular}{c|c|c}
\hline W/cm & $\begin{array}{c}\text { Cementitious material } \\
\left(\mathrm{kg} / \mathrm{m}^{3}\right)\end{array}$ & Water $\left(\mathrm{kg} / \mathrm{m}^{3}\right)$ \\
\hline 0.40 & 385 & 154.0 \\
\hline 0.35 & 450 & 157.5 \\
\hline 0.30 & 515 & 154.5 \\
\hline
\end{tabular}




\subsubsection{Validation of experimental data}

Similar to the compressive strength, free shrinkage field data were obtained as a way to validate the laboratory results. Figure 4.22 shows a comparative plot between current research data and field data for $\mathrm{WVDOH}$ class $\mathrm{H}$ concrete obtained from projects implemented during the last three years. All laboratory data are found to lie well within the field data, and the trend of laboratory results agree well with the field results.

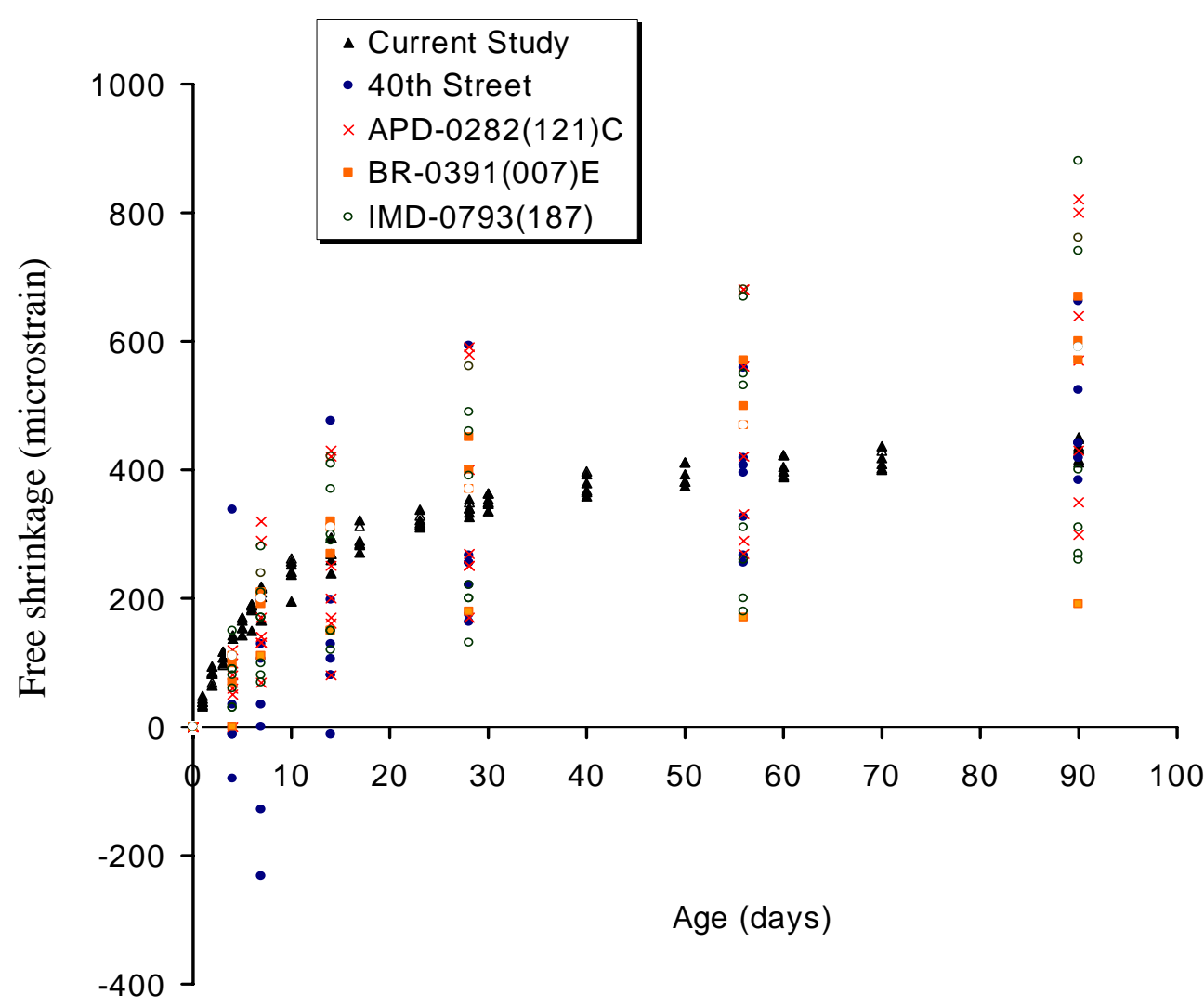

Note: Due to the different curing conditions between the current laboratory research and real life field data, the current study data in this figure has been modified from the original data on the basis of ACI 209R correction factors as listed in Appendix C.

Figure 4.22 Comparison of current research data with field data for class $\mathrm{H}$ concrete 


\subsubsection{Prediction of the free shrinkage}

There exist several models associated with the prediction of shrinkage of concrete. Among those models, the ACI 209R (1992), CEB-FIP model code (1990), and AASHTO Specification (2000) were the most widely used models in practice. The following section lists the equations for those three models as well as the prediction model for HPC proposed by Huo et al. (2001).Then, a comparison between these predictions and the present experimental data is given, followed by a proposed model developed in this study following ACI 209R.

\subsubsection{Existing models}

(a) The ACI-209R model:

$$
\varepsilon_{\text {sh }}=\frac{t}{f+t}\left(\varepsilon_{\text {shu }}\right)=\frac{t}{f+t}\left(\gamma_{s h} \varepsilon_{u}\right)
$$

where:

$t$ : the age of concrete;

$f=35$ for moisture cured concrete;

$\varepsilon_{u}$ : ultimate strain which equals to 780 as suggested by ACI -209R;

$\gamma_{s h}$ :the correction factor which is a product of different correction factors. The detail calculation methods of those correction factors can be found in ACI -209R (1992).

(b) The CEB-FIP 1990 model:

$$
\varepsilon_{s h}\left(t, t_{s}\right)=\varepsilon_{s} \beta_{R H} \beta_{s}\left(t-t_{s}\right)
$$

where:

$t$ : the age of concrete; 
$t_{s}$ : the age of concrete at the beginning of shrinkage;

$\varepsilon_{s}=\left[160-10 \beta_{s c}\left(9-f^{\prime}{ }_{c} / 10\right] \times 10^{-6}\right.$

$\beta_{\text {sc }}$ : coefficient which depends on type of cement;

$f^{\prime}{ }_{c}$ : compressive strength at 28 days;

$\beta_{R H}=-1.55 *\left(1-\left(\frac{R H}{100}\right)^{3}\right)$

$R H$ : the relative humidity of the ambient atmosphere (\%);

$\beta_{s}\left(t-t_{s}\right)=\left[\frac{\left(t-t_{s}\right)}{350(h / 100)^{2}+\left(t-t_{s}\right)}\right]^{0.5}$

h: the size factor as defined by CEB-FIP code 1990.

(c) The AASHTO Specification 2000 model:

$$
\varepsilon_{\text {sh }}=k_{s} k_{h}\left(\frac{t}{35.0+t}\right) \times 0.51 \times 10^{-3}
$$

for moist cured concrete devoid of shrinkage prone aggregates

$$
k_{s}=\left[\frac{\frac{t}{26 e^{0.0142(V / S)}+t}}{\frac{t}{45+t}}\right]\left[\frac{1064-3.70(V / S)}{923}\right]
$$

where V/S is volume to surface ratio;

$$
\begin{aligned}
& k_{h}=\frac{140-H}{70} \text { for } H<80 \% \\
& k_{h}=\frac{3(100-H)}{70} \text { for } H \geq 80 \%
\end{aligned}
$$

where $H$ is relative humidity (\%). 
(d) The model proposed by Huo et al. (2001)

Over the last decade, a number of studies have been conducted on the time-dependent properties such as free shrinkage and creep of HPC. Several prediction models for free shrinkage of HPC have been developed through modifications of the existing prediction equations for normal concrete. Those models include: Sakata et al. (2001) prediction model for drying shrinkage of concrete with a wide strength range; Garder and Lockman (2001) model for concrete with fly ash or slag blended cement and the strength up to 69 MPa; Huo et al. (2001) model for HPC with local material sources; and Lopez et al. (2004) model for HPC with lightweight aggregate. Because of the similarity of the research by Huo et al. (2001) with present study, their model was selected to compare with the data measured in this research.

The main expression in Huo's model is the same as that of ACI 209R equation (Eq. 4.14), but includes two factors $K_{s}$ and $\gamma_{s t, s}$ related to properties of HPC, as

$$
K_{s}=45-0.3626 f_{c}^{\prime}
$$

where: $f_{c}^{\prime}$ is compressive strength at 28 days in $\mathrm{MPa} ; K_{s}$ reflects the free shrinkage development tendency at early age of HPC;

$$
\gamma_{s t, s}=1.20-0.0073 f_{c}^{\prime}
$$

$\gamma_{s t, s}$ is used to account for the reduction of the ultimate free shrinkage for HPC.

Thus the Huo's model can be expressed as:

$$
\varepsilon_{s h}=\frac{t}{K_{s}+t}\left(\gamma_{s h} \varepsilon_{u}\right) \gamma_{s t, s}
$$


A comparison of predicted free shrinkage using the abovementioned models and the experimental data for HPC mixtures obtained in this study is illustrated in Figure 4.23. In which, Figure 4.23 (a) represents SL-SF HPC with w/cm =0.4, and Figure 4.23 (b) represents SL-SF HPC with w/cm = 0.3. The following comments can be made:

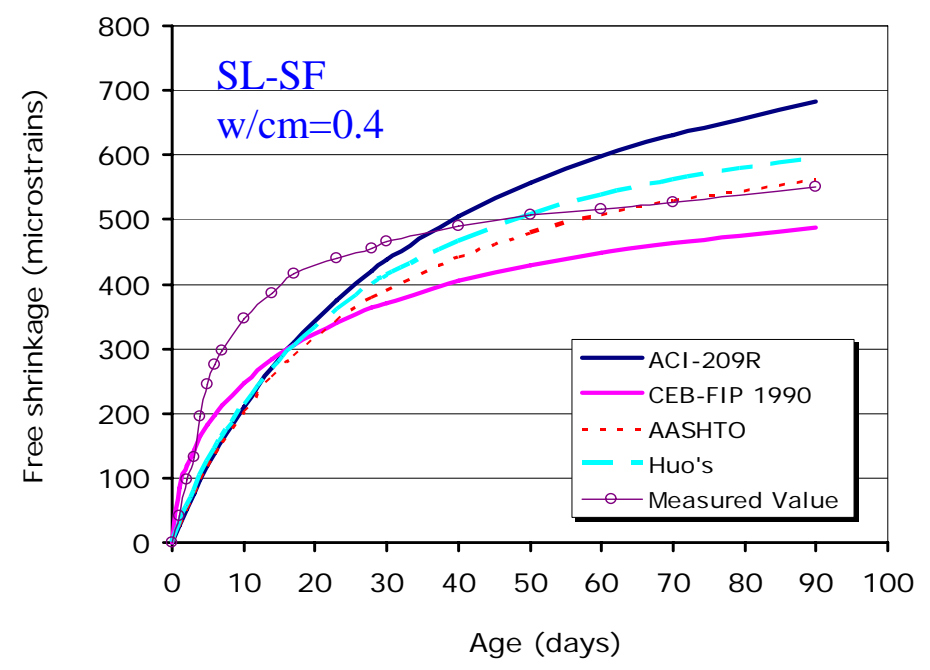

(a)

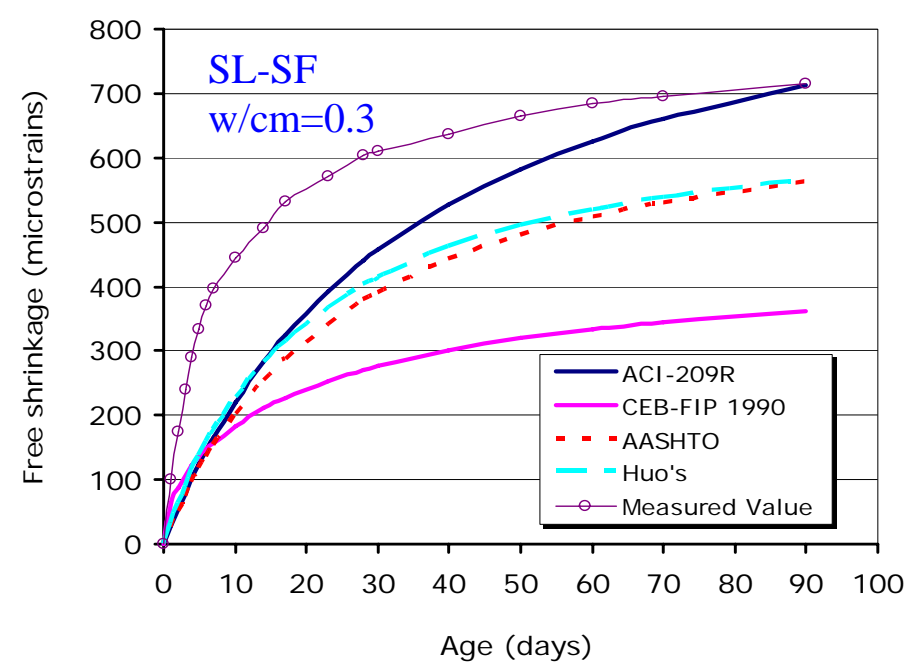

(b)

Figure 4.23 Comparison of prediction models and measured free shrinkage 
1. All the prediction models underestimate the free shrinkage at early age for HPC mixtures tested in this study.

2. For HPC with $\mathrm{w} / \mathrm{cm}=0.4$ (Figure $4.23(\mathrm{a})$ ), the free shrinkage values by AASHTO model and Huo's model were nearly equal to the measured 90-day value, while ACI 209R overestimated 90-day value and CEB-FIP underestimated 90-day value.

3. For HPC with lower w/cm = 0.3 (Figure 4.23(b)), only ACI 209R prediction value was close to the measured 90-day value, while the other models underestimated the 90-day free shrinkage.

4. Huo's model is very close to the ASSHTO model over the 90-day prediction period.

\subsubsection{Proposed model}

In this study, the inverse best-fit method as recommended by ACI 209R was adopted to find the prediction model on the basis of experimental data obtained in this research. The basic equation is the same as ACI 209R (Eq. 4.14). By inversing Eq. (4.14), the following equation is obtained:

$$
\frac{1}{\varepsilon_{\text {sh }}(t)}=\left(\frac{f}{\varepsilon_{\text {shu }}}\right) \frac{1}{t}+\frac{1}{\varepsilon_{\text {shu }}}
$$

Eq. (4.20) is a linear equation with the form of $y=a x+b$. The experimental data was manipulated guided by Eq. (4.20) to obtain the best-fit linear curve. The mixtures with the same w/cm were considered as a group. The typical plot of the best-fit results of one such group is illustrated in the Figure 4.24. From the slope and intercept of the fitting curve, the values of $f$ and $\varepsilon_{\text {shu }}$ can be calculated. Table 4.2 lists $f$ and $\varepsilon_{\text {shu }}$ values for HPC mixtures with different w/cm. 


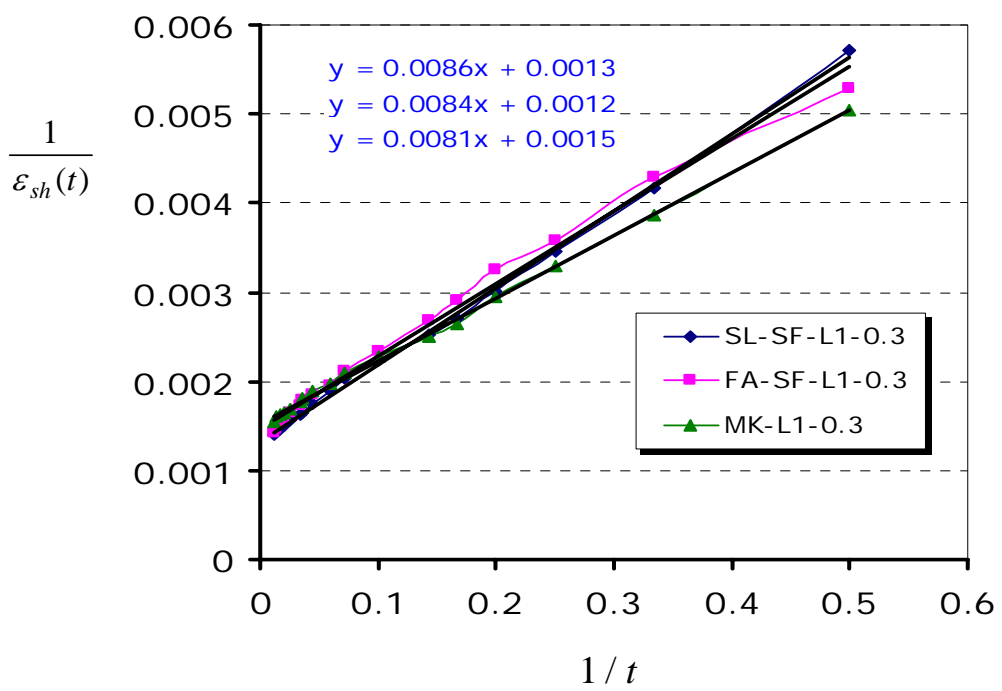

Figure 4.24 A typical plot of the best-fit curves

Table 4.2 Values of $f$ and $\varepsilon_{\text {shu }}$ for HPC mixtures with different w/cm

\begin{tabular}{c|c|c}
\hline Mixtures & $f$ & $\varepsilon_{\text {shu }}$ \\
\hline $\mathrm{w} / \mathrm{cm}=0.40$ & 10 & 590 \\
\hline $\mathrm{w} / \mathrm{cm}=0.35$ & 7.5 & 680 \\
\hline $\mathrm{w} / \mathrm{cm}=0.30$ & 6.5 & 740 \\
\hline
\end{tabular}

The proposed prediction curves as well as experimental data are plotted in Figures 4.25 (a), (b) and (c) for the mixtures with $\mathrm{w} / \mathrm{cm}=0.4,0.35$ and 0.3 , respectively. Those prediction results were used for the numerical analysis of the cracking of ring specimens in Chapter Six. 


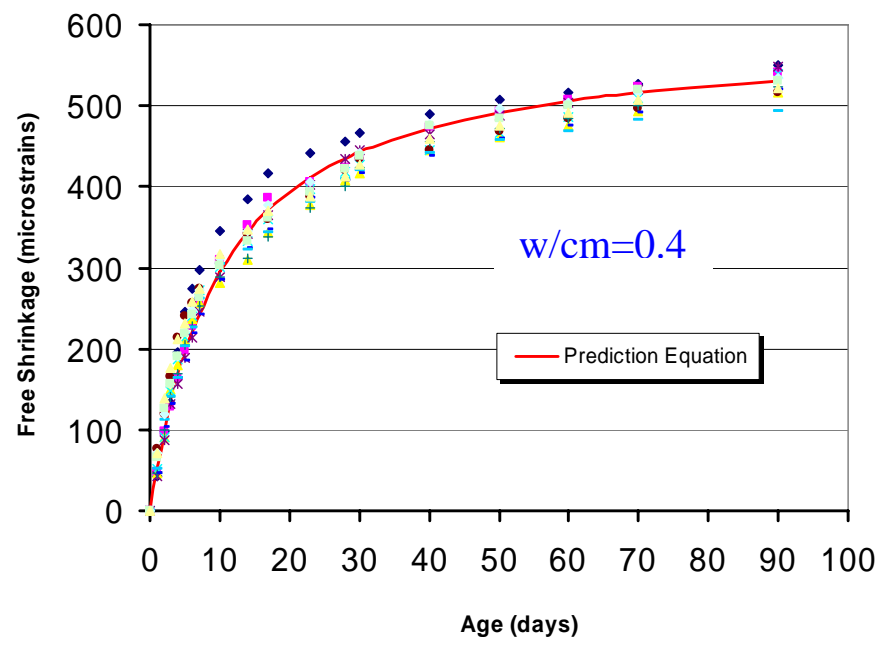

(a)

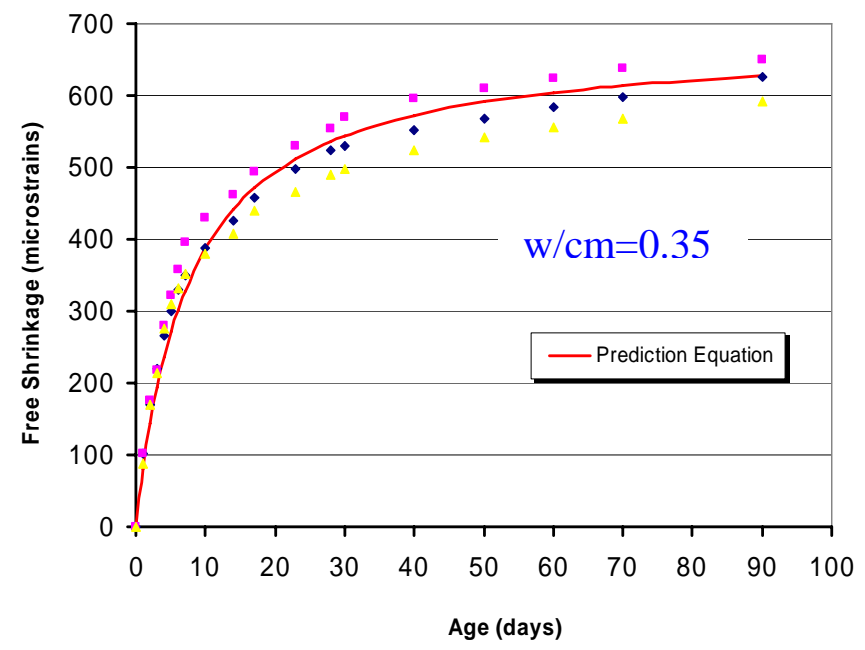

(b)

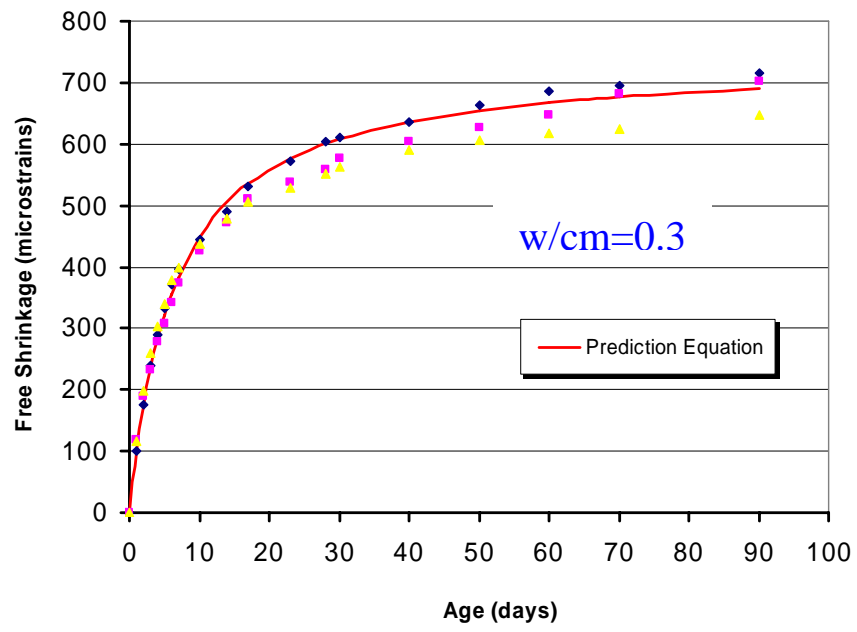

(c)

Figure 4.25 Comparative plots of proposed prediction models and experimental data 


\subsection{Concluding Remarks}

1. HPC with MK exhibited the highest compressive strength and HPC with SL-FA-SF showed the lowest compressive strength. Compressive strength increased with the decrease of w/cm at all ages.

2. The modulus of elasticity of HPC with limestone was greater than that with gravel for all SCM combinations. Modified expressions based on experimental results were proposed to correlate the modulus of elasticity with 28-day compressive strength for HPC with WV sources of aggregates.

3. The direct tensile strength increased rapidly up to 14-day, and then the increase became gradual. This may be due to the development of drying shrinkage within tensile test specimens for air curing. In some mixtures, the 90-day strength was slightly lower than the 28 day strength due to the "strength retrogression" typically exhibited by HPC.

4. A relationship between direct tensile strength and compressive strength was proposed on the basis of the test results from this study. This relationship was compared with existing correlations from other researchers and codes.

5. Tensile modulus of elasticity of all HPC tested was determined by taking into account for the influence of high stress level. This tensile modulus of elasticity value can therefore be used to analyze the cracking of structures, which takes place at high stress level. The relationships between tensile strength and tensile modulus of elasticity were also proposed.

6. The HPC with gravel had slightly lower free shrinkage than HPC with limestone. HPC with SCM (except HPC with SF) had a lower free shrinkage compared to 
control concrete (with the same cementitious material content). HPC with MK (10\%) showed the best performance in terms of free shrinkage.

7. The existing prediction models underestimate free shrinkage values for HPC at early ages. A prediction model on the basis of linear regression analysis was proposed. This model can be used to estimate the free shrinkage value of HPC at early age. 


\section{CHAPTER FIVE}

\section{RESTRAINED SHRINKAGE TEST}

\subsection{Introduction}

In the previous chapters, compressive strength, modulus of elasticity, tensile strength and free shrinkage properties of different HPC mixtures were discussed. Those properties are regarded as very important parameters that control performance of HPC. However, for structures like bridge decks, the cracking induced by restraints either due to reinforcement or the boundary conditions is of serious concern because of its detrimental effect on service-life performance. A better and more thorough understanding of cracking properties of HPC designated for bridge decks is therefore essential to anticipate or prevent crack development. To this end, the restrained shrinkage test is a useful method to evaluate the cracking potential of HPC mixtures.

As it was discussed in Chapter Two, three different types of restrained shrinkage testing methods (bar, slab and ring specimen) are available. Among these testing methods, researchers are increasingly using the ring test (Grzybowski and Shah, 1990; Wiegrink et al., 1996; Folliard and Berke, 1997), because the test setup is relatively simple and easy to implement; additionally, the ring test can provide sustained and sufficient restraint to induce the cracking for common size specimens. Also, for analysis purpose, ring specimen has the advantage of being axisymmetric.

In 1998, AASHTO adopted the ring specimen test as a provisional standard test (AASHTO 1998). Recently, several researchers have used the ring test to investigate 
restrained properties of concrete. Some of the studies include: Li et al. (1999) used the ring specimen to study the crack width of HPC. Whitting et al. (2000) investigated the cracking tendency of silica fume modified bridge deck concrete. See et al. (2003) employed an instrumented AASHTO ring to quantify restrained shrinkage behavior with a focus on the tensile creep properties. Subramanian et al. (2005) investigated the ultrafine fly ash modified concrete with the ring specimen.

In this study, the AASHTO type ring specimen test was adopted to investigate the restrained cracking behaviors of a number of HPC mixtures with different strength, modulus of elasticity, and free shrinkage properties. A cracking onset prediction model was proposed for the evaluation of cracking properties of different HPC mixtures. On the basis of the test results, the best-performing HPC mixtures were selected and recommended for field applications by the WVDOH.

\subsection{Testing Procedure}

The testing was conducted per "Standard Practice for Estimating the Cracking Tendency of Concrete,” which was first proposed by AASHTO in 1998 as a provisional standard (AASHTO, 1998) with some modifications. The details of the test are described as follows:

(a) The configuration of the ring specimen is shown in Figure 5.1. It was made of a concrete ring cast around a steel ring. The outside diameter of the concrete ring was 457 mm (18 in) and the outside and inside diameters of the steel ring were $305 \mathrm{~mm}$ (12 in) and $280 \mathrm{~mm}$ (11 in), respectively. The inner and outer faces of the steel ring were machined to a smooth polished surface. The height of the ring was $152 \mathrm{~mm}$ (6 in). 
(b) Four strain gauges were used to record the strain inside the steel ring and automatically capture the cracking onset time. Strain gauges were bonded at four equidistant mid-height locations on the interior of the steel ring.

(c) The types of mixtures in this test are the same as those used for the direct tensile and free shrinkage tests. For each mixture, two replicate rings were cast. The preparation of the concrete followed the relevant requirements of AASHTO T126. A total of 36 ring specimens were tested

(d) The concrete ring was cast within an exterior cardboard mold and steel ring, placed over a plywood board. After 24 hours curing under wet burlap, the specimens were demolded and coated with silicone rubber sealant on the top surface. All the specimens were moved to a controlled environmental chamber with a constant air temperature of 23 ${ }^{\circ} \mathrm{C}\left(73^{\circ} \mathrm{F}\right)$ and relative humidity of $50 \%$, which was the same as the environmental conditions for direct tensile and free shrinkage tests.

(e) A data acquisition system was used in this study. The strain gauge reading was recorded automatically every 30 minutes.

(f) Once the cracking occurred, the crack width was measured using a crack detection microscope having $0.02 \mathrm{~mm}$ accuracy. The crack width recorded was an average of three measurements: one at the center of the outside surface of the ring, one at one inch from top, and one at one inch from bottom. The tests were conducted until 90 days.

Figure 5.2 shows a typical specimen with strain gauges bonded to the interior of the steel ring, and Figure 5.3 shows an overview of the test setup in an environmental chamber including a data acquisition system. 


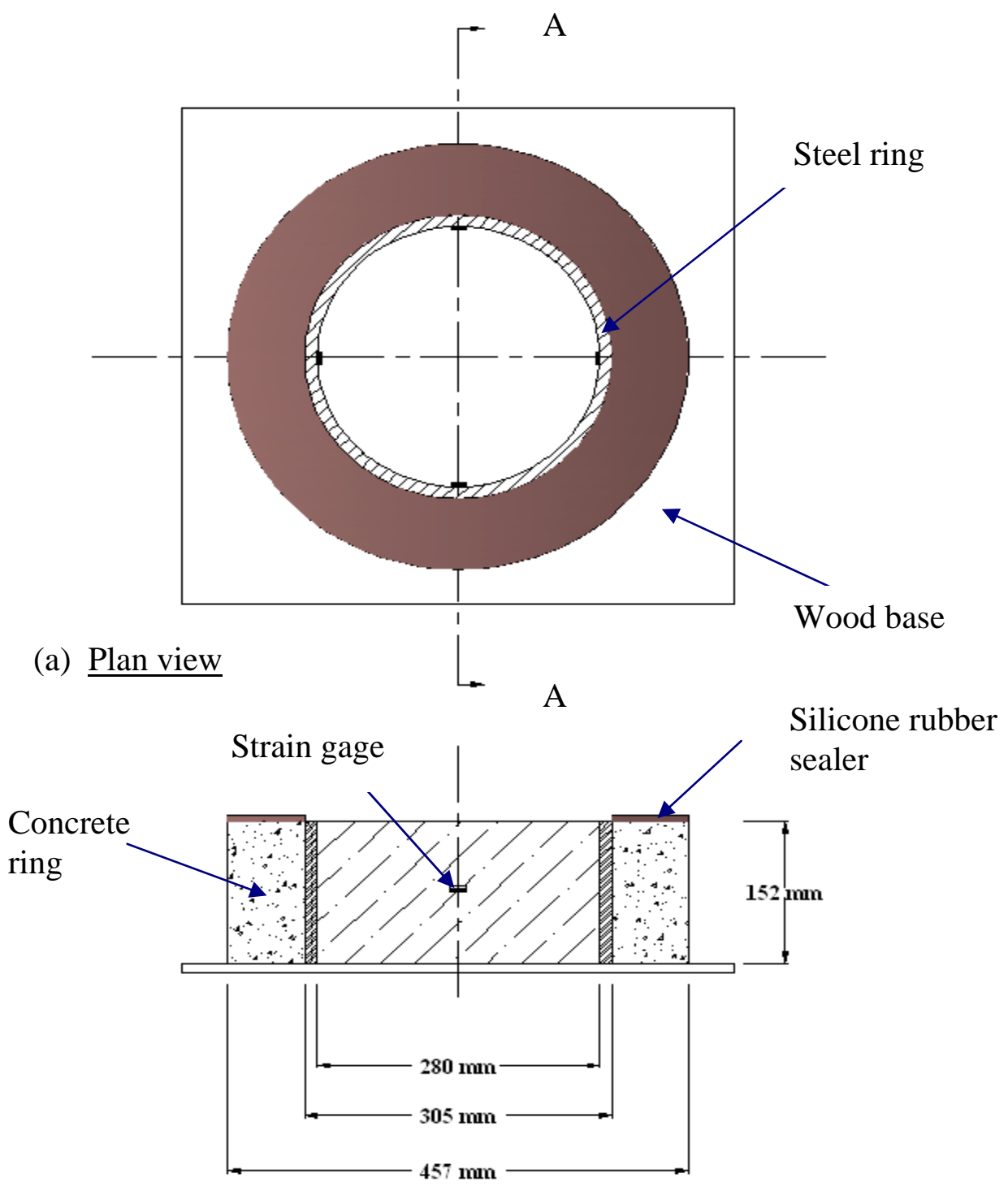

(b) Section A-A

$$
(1 \mathrm{~mm}=0.03937 \mathrm{in})
$$

Figure 5.1 Schematic view of a ring specimen 


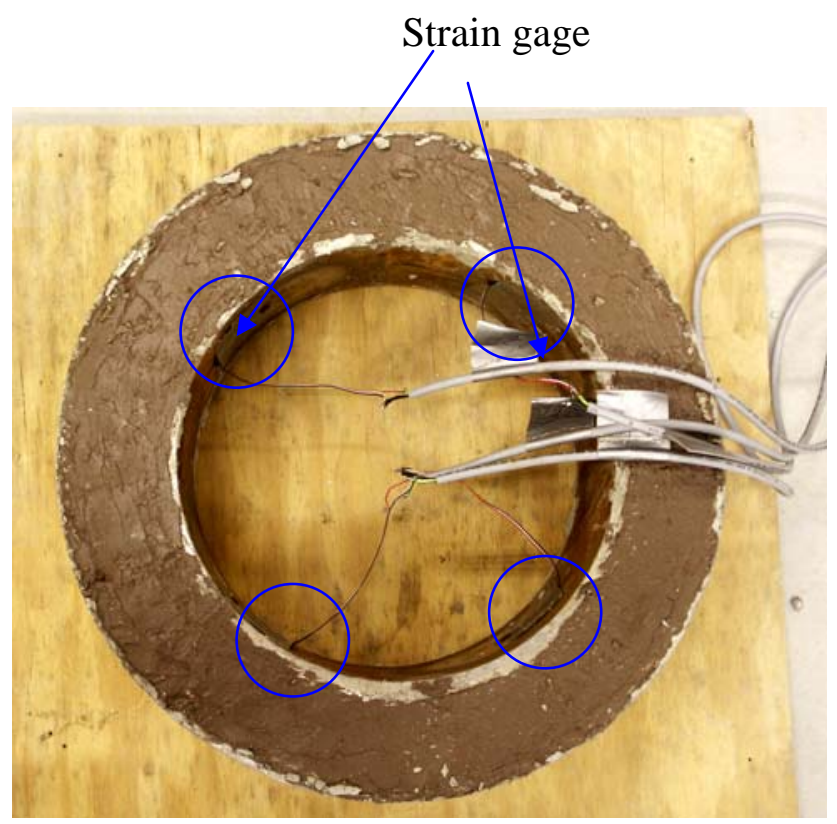

Figure 5.2 Close-view of a typical ring specimen with four strain gages

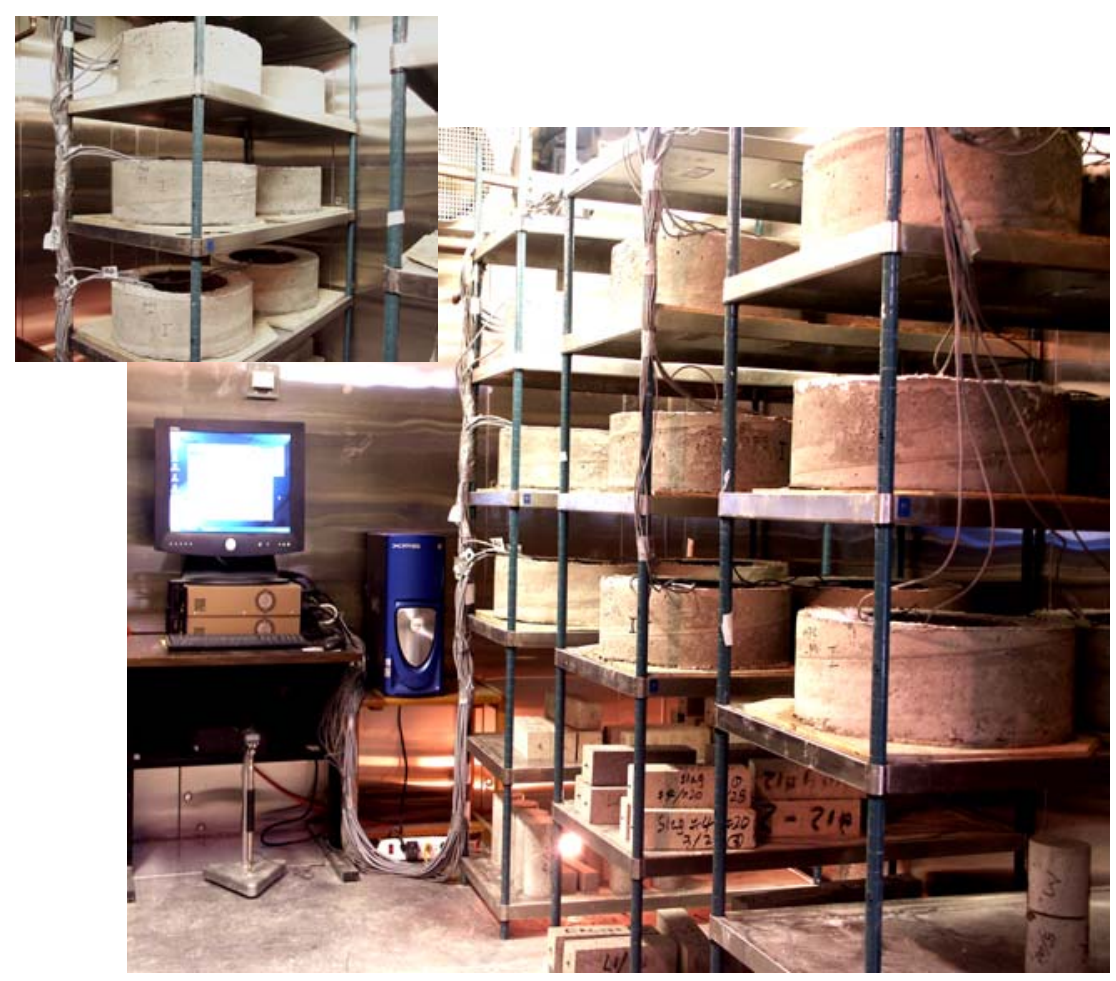

Figure 5.3 Overview of test setup in an environmental chamber and data acquisition system 


\subsection{Results and Discussions}

\subsubsection{Strain of steel ring with time}

Since the concrete ring is restrained against horizontal movement in the radial direction by the steel ring, as the concrete shrinks, the internal pressure $p$ will develop between concrete ring and steel ring, and the steel ring will experience deformation under compression. The interaction of the steel ring and concrete ring can be illustrated in Figure 5.4.

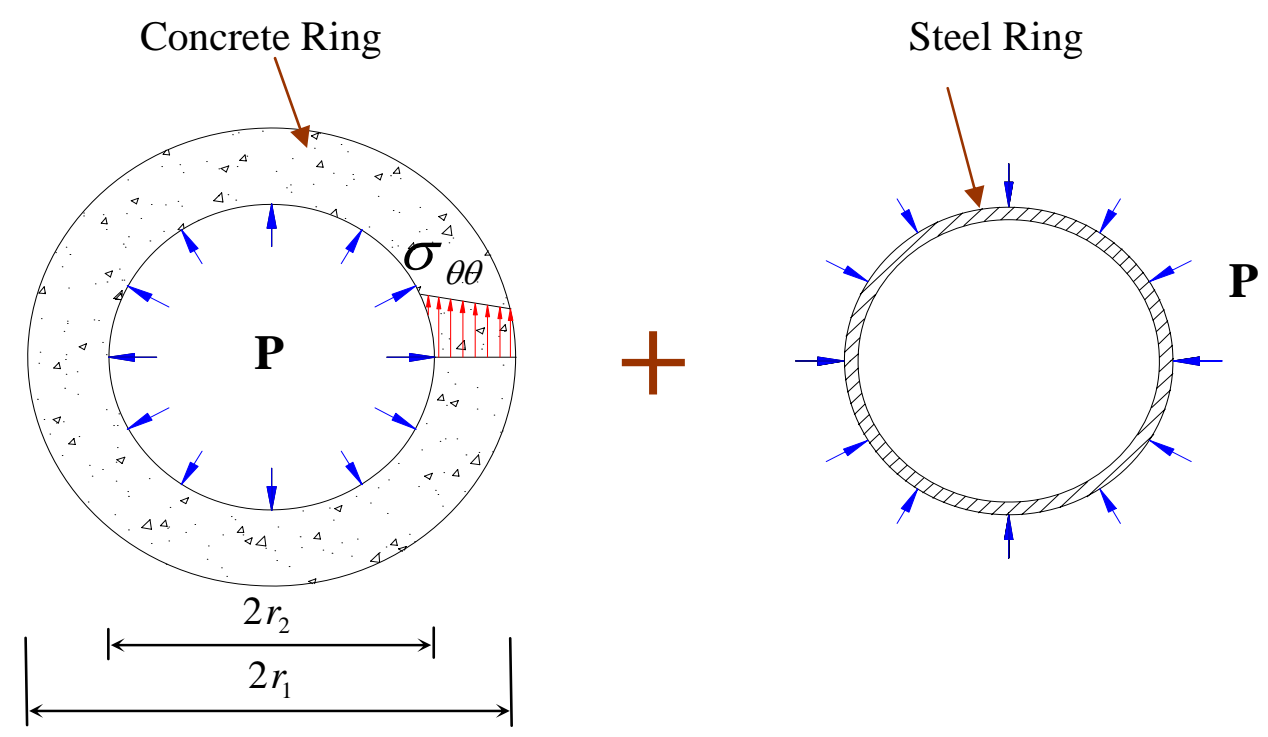

Figure 5.4 Illustration of interaction between concrete ring and steel ring

Before the crack occurred in the concrete ring, the concrete ring and steel ring can be considered as an elastic body. Based on the generalized elastic solutions (Timoshenko and Goodier, 1970) for stress analysis of a ring under internal and external pressure, the following equations for circumferential and radial stresses can be defined: 


$$
\begin{gathered}
\sigma_{\theta \theta}=\frac{-r_{1}^{2} r_{2}^{2}\left(p_{e}-p_{i}\right)}{\left(r_{1}^{2}-r_{2}^{2}\right) r^{2}}+\frac{r_{2}^{2} p_{i}-r_{1}^{2} p_{e}}{\left(r_{1}^{2}-r_{2}^{2}\right)} \\
\sigma_{r r}=\frac{r_{1}^{2} r_{2}^{2}\left(p_{e}-p_{i}\right)}{\left(r_{1}^{2}-r_{2}^{2}\right) r^{2}}+\frac{r_{2}^{2} p_{i}-r_{1}^{2} p_{e}}{\left(r_{1}^{2}-r_{2}^{2}\right)}
\end{gathered}
$$

where: $r_{1}$ and $r_{2}$ are external and internal radii, respectively; $p_{e}$ and $p_{i}$ are external and internal pressures, respectively; $\sigma_{\theta \theta}$ and $\sigma_{r r}$ are circumferential and radial stress, respectively. For the concrete ring in this study, the external pressure $p_{e}$ does not exist. Eq. (5.1) and Eq. (5.2) then can be rewritten as:

$$
\begin{gathered}
\sigma_{\theta \theta}=\frac{\left(r_{1}^{2} / r^{2}+1\right) p}{r_{1}^{2} / r_{2}^{2}-1} \\
\sigma_{r r}=\frac{\left(r_{1}^{2} / r^{2}-1\right) p}{r_{1}^{2} / r_{2}^{2}-1}
\end{gathered}
$$

It can be seen that the concrete ring is under the condition of biaxial tension and compression. According to Hooker's law, the relationship between the circumferential strain inside the concrete ring and internal pressure can be obtained as:

$$
\varepsilon_{\theta \theta}=\frac{p}{E}\left(\left(r_{1}^{2}+r_{2}^{2}\right) /\left(r_{1}^{2}-r_{2}^{2}\right)+\mu\right)
$$

where: $\mu$ is Poisson's ratio, and $E$ is modulus of elasticity.

$\varepsilon_{\theta \theta}$ can be regarded as the shrinkage induced strain. Since the concrete ring and steel ring work together until the cracking happens, the strain development inside the concrete ring can be approximately known from the strain gages bonded on the interior surface of the steel ring. Furthermore, from Eq. (5.3) and Eq. (5.4), the stress accumulated inside the concrete ring is also known. 
Table 5.1 Stresses and strains inside concrete rings

\begin{tabular}{c|c|c|c|c|c}
\hline $\begin{array}{c}\text { Mixtures } \\
(1)\end{array}$ & $\begin{array}{c}\text { Max. strain } \\
\text { measured } \\
\left(\varepsilon_{\theta \theta} 10^{-6}\right) \\
(2)\end{array}$ & $\begin{array}{c}\text { Max. stress } \\
\text { accumulated } \\
\left(\sigma_{\theta \theta} \text { MPa }\right) \\
(3)\end{array}$ & $\begin{array}{c}\text { Tensile } \\
\text { strength of } \\
\text { concrete at } \\
\text { the onset of } \\
\text { cracking } \\
\left(f_{t} \text { MPa }\right) \\
(4)\end{array}$ & $\begin{array}{c}\text { Free shrinkage } \\
\text { at the onset of } \\
\text { cracking } \\
\left(\varepsilon_{\text {free }} 10^{-6}\right) \\
(5)\end{array}$ & $\begin{array}{c}\text { Creep } \\
\text { effect } \\
\varepsilon_{\text {free }} \\
\varepsilon_{\theta \theta} \\
(6)\end{array}$ \\
\hline SL-SF-L1-0.4 & 63 & 1.368 & 2.546 & 375 & 6.0 \\
\hline FA-SF-L1-0.4 & 67 & 1.491 & 2.618 & 342 & 5.1 \\
\hline MK-L1-0.4 & 65 & 1.514 & 2.745 & 296 & 4.6 \\
\hline SL-SF-L1-0.35 & 76 & 1.886 & 2.950 & 389 & 5.1 \\
\hline FA-SF-L1-0.35 & 75 & 1.870 & 2.954 & 439 & 5.9 \\
\hline MK-L1-0.35 & 82 & 2.141 & 3.123 & 394 & 4.8 \\
\hline SL-SF-L1-0.3 & 82 & 2.070 & 3.019 & 412 & 5.0 \\
\hline FA-SF-L1-0.3 & 80 & 2.039 & 3.040 & 409 & 5.1 \\
\hline MK-L1-0.3 & 85 & 2.220 & 3.124 & 412 & 4.8 \\
\hline SL-SF-L2-0.4 & 63 & 1.527 & 2.883 & 342 & 5.4 \\
\hline FA-SF-L2-0.4 & 69 & 1.686 & 2.886 & 357 & 5.2 \\
\hline MK-L2-0.4 & 65 & 1.614 & 2.927 & 341 & 5.2 \\
\hline SL-SF-G1-0.4 & 56 & 1.161 & 2.497 & 344 & 6.1 \\
\hline FA-SF-G1-0.4 & 58 & 1.226 & 2.572 & 367 & 6.3 \\
\hline MK-G1-0.4 & 60 & 1.318 & 2.702 & 349 & 5.8 \\
\hline SL-SF-G2-0.4 & 65 & 1.452 & 2.724 & 350 & 5.4 \\
\hline FA-SF-G2-0.4 & 58 & 1.138 & 2.290 & 347 & 6.0 \\
\hline MK-G2-0.4 & 60 & 1.382 & 2.819 & 362 & 6.0 \\
\hline
\end{tabular}

Table 5.1 shows the results of strain and stress analysis of different HPC rings at the onset of cracking. The second column of Table 5.1 is the average value of maximum strains recorded by strain gauges bonded on the interior surface of steel rings. In this study, soon after the first 24 hours of wet burlap curing (100\% relative humidity), the ring specimens were maintained in a controlled environment $\left(23^{\circ} \mathrm{C}\right.$ and relative humidity of 50\%). The autogenous shrinkage during the time from initial setting of the concrete to the onset of drying was not included in the strain reading. However, according to the study conducted by Heather et al.(2003), the strain caused by initial autogenous shrinkage 
of the concrete is usually very low with typical values in the range of $0 \sim 8 \mu \varepsilon$.

The third column of Table 5.1 represents the maximum stress accumulated inside the concrete ring, which was calculated using Eq. (5.3) and Eq. (5.4) with the assumption that the level of strain inside the steel ring was the same as the strain inside the concrete ring.

The fourth column is tensile strength of concrete materials at the time of cracking onset. By comparing the third column with the fourth column, it is observed that, the maximum stress inside the concrete ring was much lower than the tensile strength developed when the crack occurred. This indicates that the traditional strength method is not suitable for the analysis or prediction of failure of the ring specimens.

The sixth column of Table 5.1 is the so-called creep effect, which is quantified by dividing the actual free shrinkage (column 5) by the measured strain inside the steel ring, or concrete ring (column 2). The value in column 6, while small, shows the beneficial effect of creep or stress relaxation on the cracking mitigation of the concrete specimen.

Figure 5.5 shows typical strains inside steel rings versus time. Figure 5.5 (a) shows the HPC mixtures with limestone and different w/cm, and Figure 5.5 (b) shows the HPC mixtures with different coarse aggregates and $\mathrm{w} / \mathrm{cm}=0.4$. The strain data was plotted at one-day time intervals in those two figures.

The maximum strain developed in the steel ring was around $55 \sim 85 \mu \varepsilon$ as shown in Table 5.1. Figure 5.5 (b) shows that there are minor differences between the limestone and gravel HPC for maximum strains, but the onset of cracking occurred early for limestone. However, for HPC with different w/cm (Figure 5.5 (a)), the maximum strain was higher and the slope of the curve was steeper with the decrease in $w / c m$. As 
illustrated in Figure 5.5, an abrupt jump in the strain indicated the onset of cracking of the concrete ring specimen.

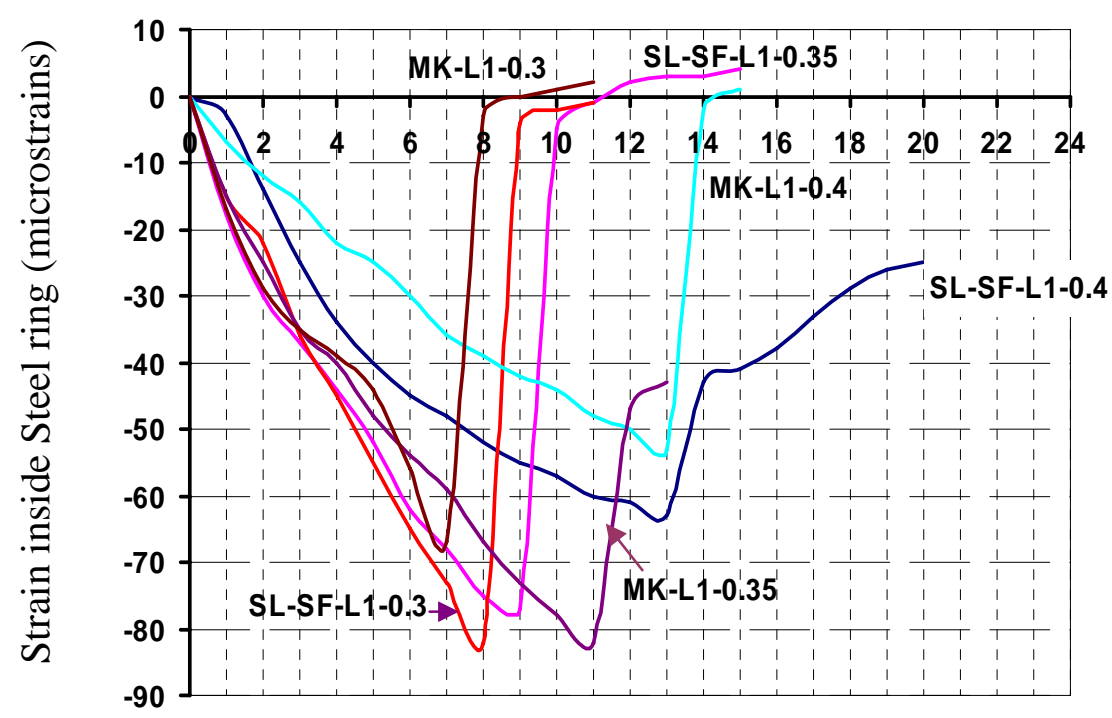

Time after initiation of drying (days)

(a)

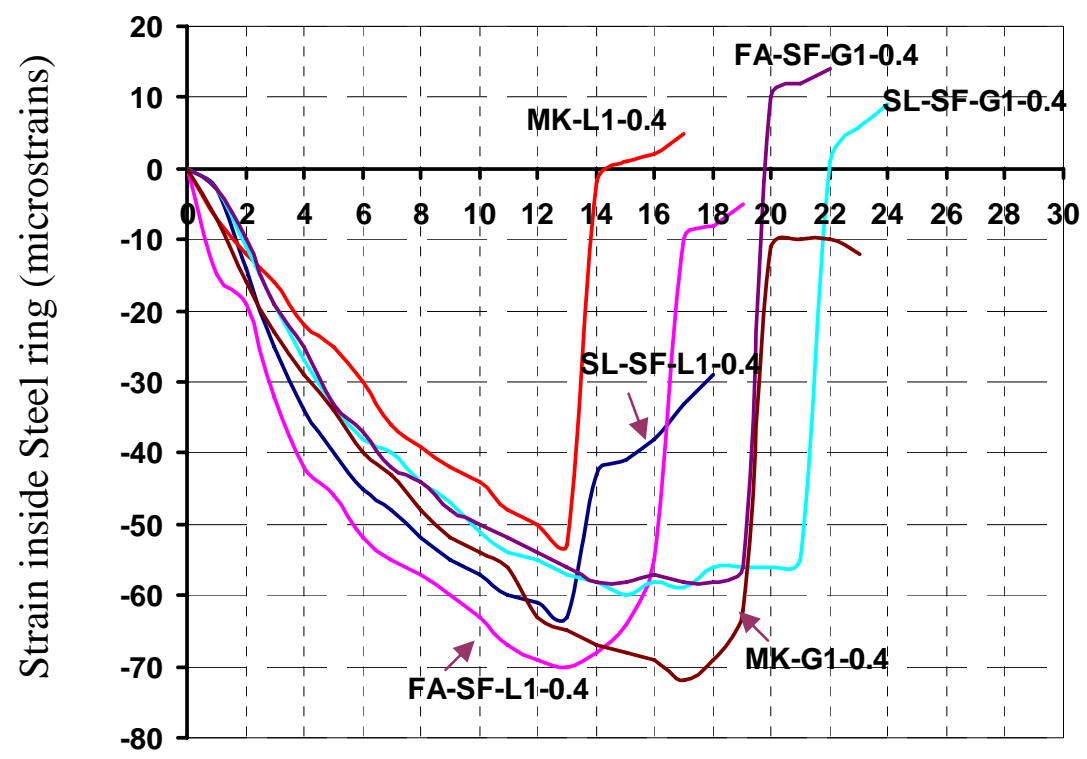

Time after initiation of drying (days)

(b)

Figure 5.5 Strain developments with time inside the steel ring 


\subsubsection{Cracking onset day}

Out of a total of 36 specimens, 35 developed visible cracks. Two-thirds (23 specimens) of the specimens had two cracks on diametrically opposite sides, and onethird (12 specimens) had cracks on one side only.

Table 5.2 reports the onset of cracks (in days) for all the HPC mixtures investigated in this study. Figure 5.6 shows some typical cracks on the surface of the ring specimens.

From the results, it may be concluded that the AASHTO ring test instrumented with the strain gauges can capture the cracking time with reasonable accuracy (virtually within one minute). This test allows for an effective way to evaluate the cracking properties of different concrete mixtures.

Table 5.2 Cracking onset days for different HPC mixtures

\begin{tabular}{|c|c|c|c|}
\hline \multirow{2}{*}{ Mixtures } & \multicolumn{3}{|c|}{ Onset of cracking (days) } \\
\hline & Specimen 1 & Specimen 2 & Average \\
\hline SL-SF-L1-0.4 & 14 & 12 & 13 \\
\hline FA-SF-L1-0.4 & 11 & 15 & 13 \\
\hline MK-L1-0.4 & 11 & 13 & 12 \\
\hline SL-SF-L2-0.4 & 13 & 16 & 15 \\
\hline FA-SF-L2-0.4 & 16 & $-*$ & 16 \\
\hline MK-L2-0.4 & 13 & 15 & 14 \\
\hline SL-SF-G1-0.4 & 15 & 21 & 18 \\
\hline FA-SF-G1-0.4 & 21 & 19 & 20 \\
\hline MK-G1-0.4 & 17 & 19 & 18 \\
\hline SL-SF-G2-0.4 & 21 & 16 & 19 \\
\hline FA-SF-G2-0.4 & 19 & 15 & 17 \\
\hline MK-G2-0.4 & 16 & 24 & 20 \\
\hline SL-SF-L1-0.35 & 9 & 11 & 10 \\
\hline FA-SF-L1-0.35 & 10 & 11 & 11 \\
\hline MK-L1-0.35 & 13 & 11 & 12 \\
\hline SL-SF-L1-0.3 & 8 & 8 & 8 \\
\hline FA-SF-L1-0.3 & 8 & 10 & 9 \\
\hline MK-L1-0.3 & 8 & 7 & 8 \\
\hline
\end{tabular}

Note $^{*}$ the specimen experienced no crack 

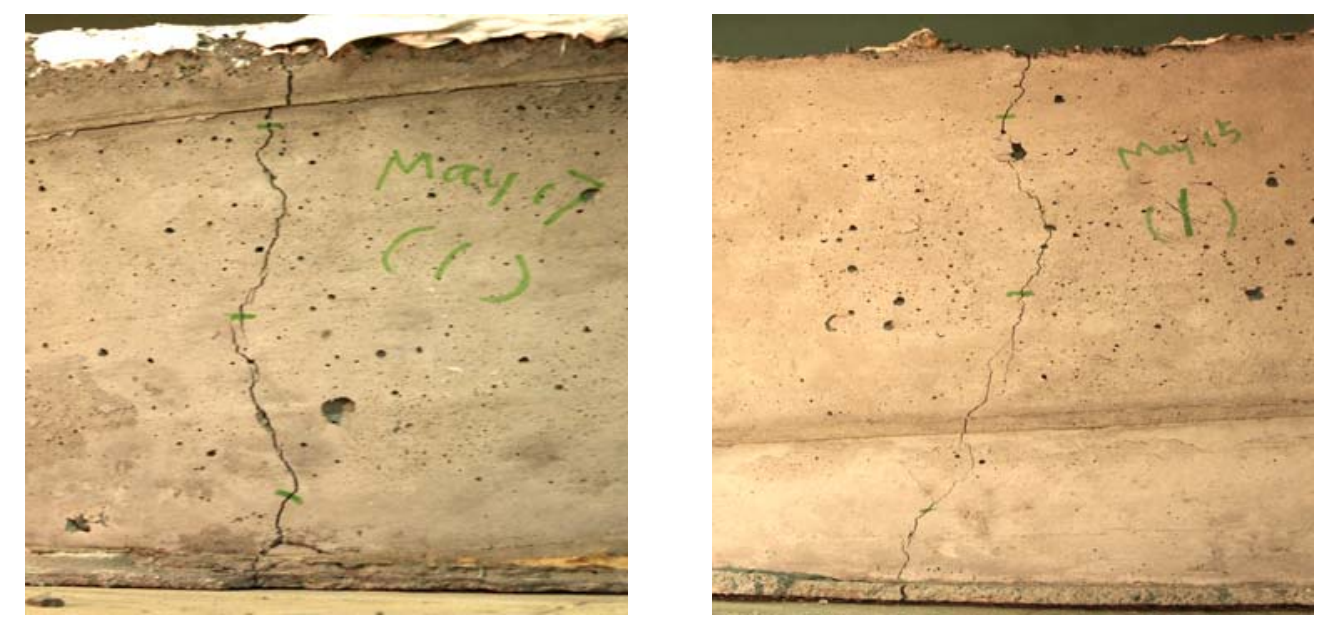

Figure 5.6 Typical cracks on the surface of the concrete rings

Figure 5.7 (a) shows the crack onset of HPC mixtures having different coarse aggregates for $\mathrm{w} / \mathrm{cm}=0.4$. It is observed that the mixtures with gravel generally cracked 2 8 days later than the mixtures with limestone under the same conditions. As previously discussed, gravel mixtures had almost the same total free shrinkage compared to limestone mixtures. The modulus of elasticity of gravel mixtures was $10 \% \sim 15 \%$ lower than that of limestone mixtures. Also, it is believed (Neville, 1996) that the creep relaxation of concrete with gravel was greater than concrete with limestone at early ages. The lower modulus of elasticity and higher creep relaxation can explain why mixtures with gravel had a better crack resisting performance than mixtures with limestone.

Figure 5.7 (b) shows the effect of w/cm on the onset of cracking. It is observed that at lower w/cm, the crack occurred earlier. This was expected since the mixtures with lower w/cm had higher volume of cementitious paste and lower aggregate content, which led to higher free shrinkage. Also, the lower w/cm mixtures had higher modulus of elasticity which reduced the strain capacity of the HPC. 


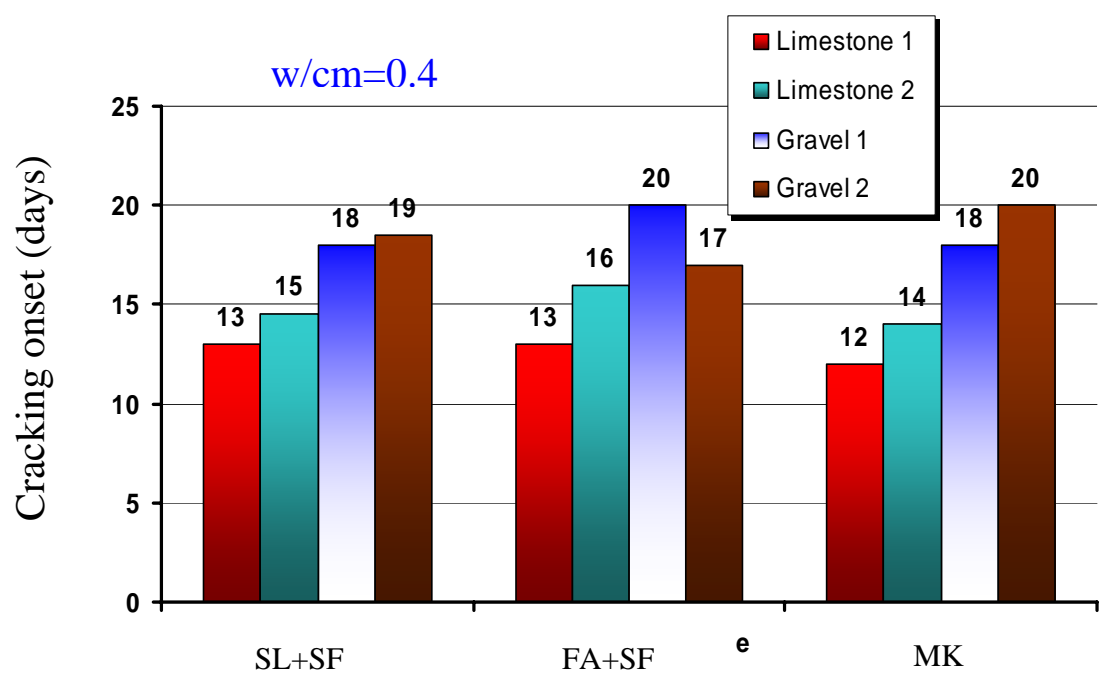

(a)

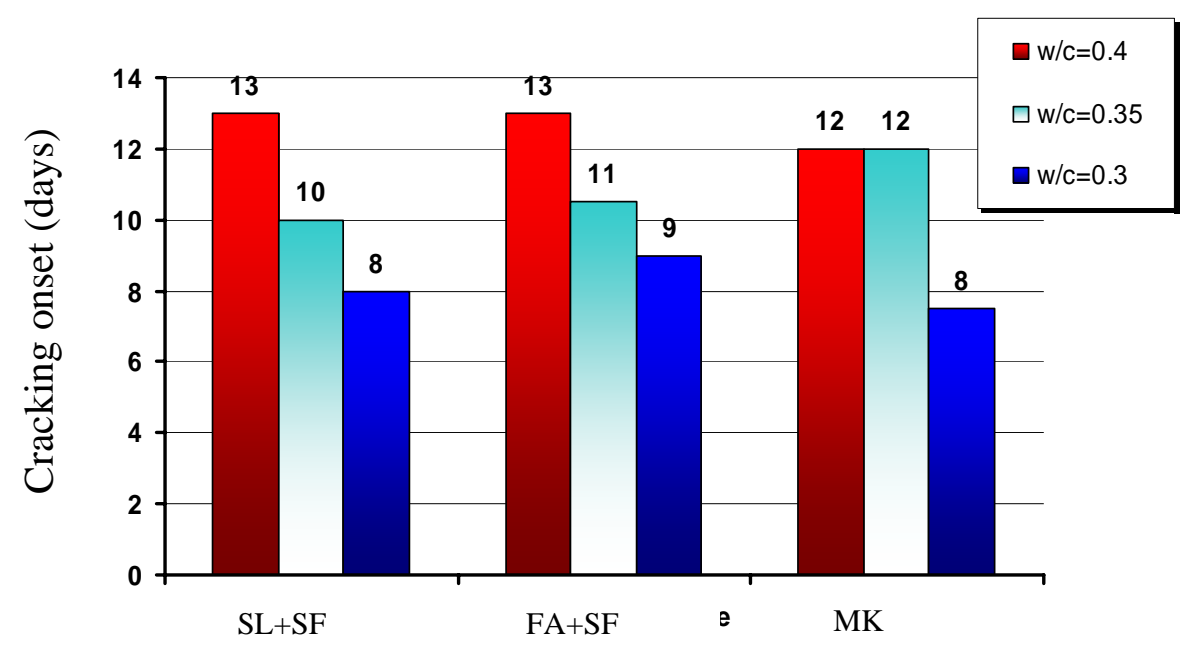

(b)

Figure 5.7 Comparative plots of cracking onset for different HPC mixtures 


\subsubsection{Crack widths with time}

As mentioned before, the crack width on the surface of the ring specimen was measured by using a microscope. The measurement was done every three days for the first two weeks, then every ten days until 90 days. The average of three values measured at three different positions was taken as the crack width. For the ring specimens with two cracks at opposite locations, the sum of these two crack widths was taken as the total crack width. Figure 5.8 shows the development of crack widths with time for typical HPC mixtures tested in this study.

The crack width increased rapidly in the first few days for all the mixtures. After about ten days from crack-initiation time, the increase in crack width tended to be slower and finally reached to a stable condition. For the mixtures with lower w/cm $(\mathrm{w} / \mathrm{cm}=0.3$ and $\mathrm{w} / \mathrm{cm}=0.35$ ), the crack width developed faster compared to mixtures with $\mathrm{w} / \mathrm{cm}=$ 0.4 .

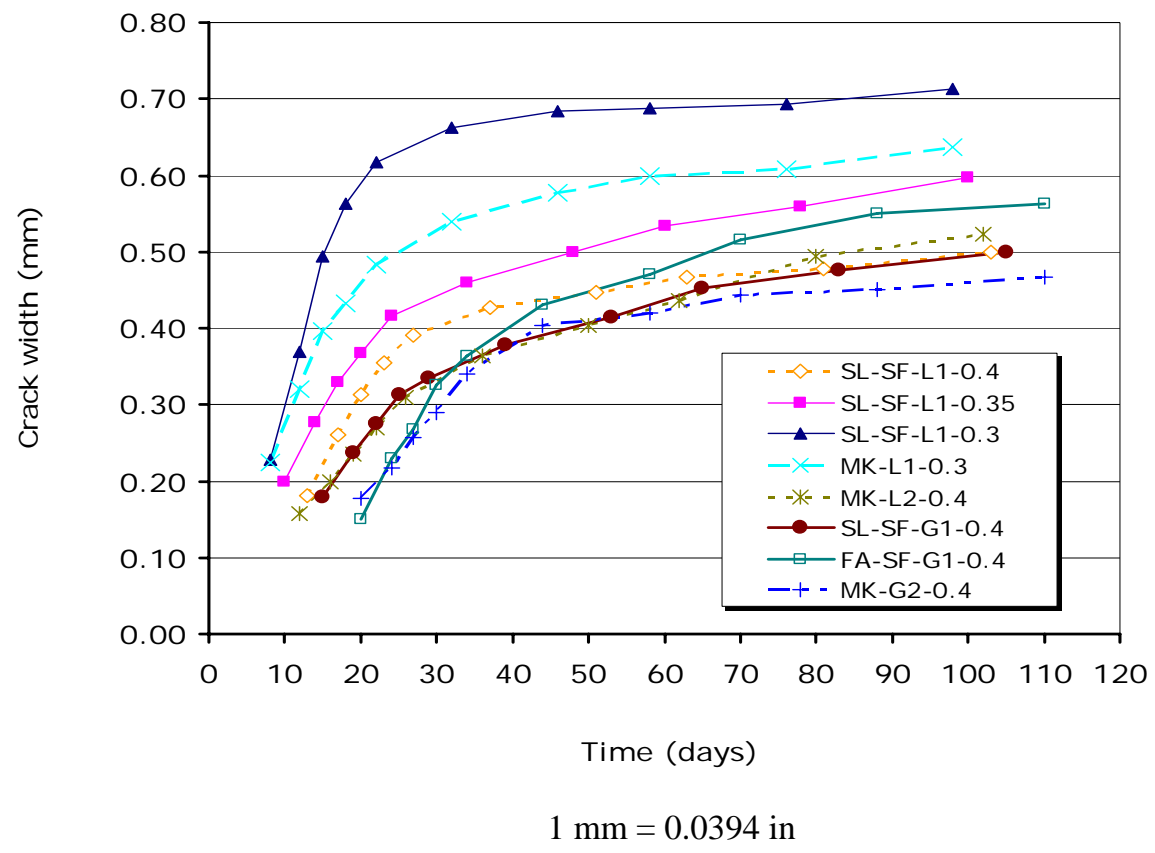

Figure 5.8 The development of crack widths with time of different HPC mixtures 


\subsection{Prediction of Cracking Onset}

In the bridge construction practice, the cracking of bridge deck is a major concern. As it is discussed in Chapter Two, the cracking of bridge deck is a complex process. Many parameters such as free shrinkage, tensile creep relaxation, tensile strength, modulus of elasticity, and fracture properties can influence the cracking behavior. It is essential to develop an effective method to evaluate the cracking tendency of different concrete mixtures in order to design crack resistant concrete the crack sensitive structures like bridge decks.

The AASHTO ring specimen test provides an easy and effective way to evaluate the cracking properties. But a well controlled environmental chamber, data acquisition system, and precise workmanship are needed to successfully conduct the test. Not all the laboratories have this kind of facilities. Also unless a good quality control is followed, the repeatability of the results will not be achieved.

Some researchers like Shah et al. (1998) developed a numerical method to predict the shrinkage cracking of concrete. However, this method requires information on some material parameters, such as tensile creep, fracture properties, etc., which are very difficult to obtain and still unfamiliar to most construction industries.

For today's industrial practice, the 28-day compressive strength and modulus of elasticity are the most common parameters used to evaluate the concrete properties. In bridge construction, the 90-day free shrinkage data is required for bridge deck concrete mixtures by most state DOTs. Based on the above practical considerations, a simplified approach is recommended to evaluate cracking properties of different concrete mixtures, 
from most common material parameters such as compressive strength, modulus of elasticity, and free shrinkage.

Table 5.3 lists the 28-day compressive strength, 28-day modulus of elasticity, 90-day free shrinkage, and cracking onset days for all HPC mixtures tested in this study. Based on this data set, a regression analysis was performed to find the best fitting relationship among those parameters. Figure 5.9 furnishes the regression result with $R^{2}=0.81$. A relationship between cracking onset in days and a combined function, designated here as cracking index, which included compressive strength, modulus of elasticity, and free shrinkage was established.

Table 5.3 Basic material parameters and cracking onset data of all HPC mixtures tested

\begin{tabular}{|c|c|c|c|c|c|c|}
\hline \multirow{2}{*}{ Mixtures } & \multirow{2}{*}{$\begin{array}{c}\text { 28-d } \\
\text { Comp. } \\
\text { strength } \\
\text { (MPa) }\end{array}$} & \multirow{2}{*}{$\begin{array}{l}\text { Modulus of } \\
\text { elasticity } \\
(\mathrm{GPa})\end{array}$} & \multirow{2}{*}{$\begin{array}{l}\text { 90-day } \\
\text { free strain } \\
\qquad(\mu \varepsilon)\end{array}$} & \multicolumn{3}{|c|}{ Cracking onset day (days) } \\
\hline & & & & $\begin{array}{c}\text { Measured } \\
\# 1\end{array}$ & $\begin{array}{c}\text { Measured } \\
\# 2\end{array}$ & Predicted \\
\hline SL-SF-L1-0.4 & 53.8 & 34.3 & 550 & 12 & 14 & 11.5 \\
\hline FA-SF-L1-0.4 & 51.5 & 32.5 & 541 & 11 & 15 & 12.6 \\
\hline MK-L1-0.4 & 57.2 & 34.4 & 517 & 11 & 13 & 12.5 \\
\hline SL-SF-L2-0.4 & 55.5 & 31.3 & 539 & 13 & 16 & 13.5 \\
\hline FA-SF-L2-0.4 & 54.3 & 30.4 & 549 & 16 & $-*$ & 13.8 \\
\hline MK-L2-0.4 & 63.3 & 33.8 & 517 & 13 & 15 & 12.9 \\
\hline SL-SF-G1-0.4 & 48.6 & 25.6 & 524 & 15 & 21 & 18.6 \\
\hline FA-SF-G1-0.4 & 48.9 & 23.1 & 520 & 19 & 21 & 21.8 \\
\hline MK-G1-0.4 & 54.1 & 26.6 & 493 & 17 & 19 & 19.1 \\
\hline SL-SF-G2-0.4 & 49.0 & 26.0 & 534 & 16 & 21 & 17.7 \\
\hline FA-SF-G2-0.4 & 45.7 & 25.9 & 531 & 15 & 19 & 17.8 \\
\hline MK-G2-0.4 & 53.3 & 26.5 & 522 & 16 & 24 & 17.8 \\
\hline SL-SF-L1-0.35 & 64.0 & 34.2 & 627 & 9 & 10 & 10.1 \\
\hline FA-SF-L1-0.35 & 56.9 & 33.7 & 650 & 10 & 11 & 9.7 \\
\hline MK-L1-0.35 & 71.4 & 33.9 & 592 & 11 & 12 & 11.1 \\
\hline SL-SF-L1-0.3 & 68.2 & 34.7 & 716 & 8 & 8 & 8.4 \\
\hline FA-SF-L1-0.3 & 68.5 & 33.6 & 702 & 8 & 10 & 9.1 \\
\hline MK-L1-0.3 & 86.3 & 35.1 & 648 & 7 & 8 & 9.7 \\
\hline
\end{tabular}




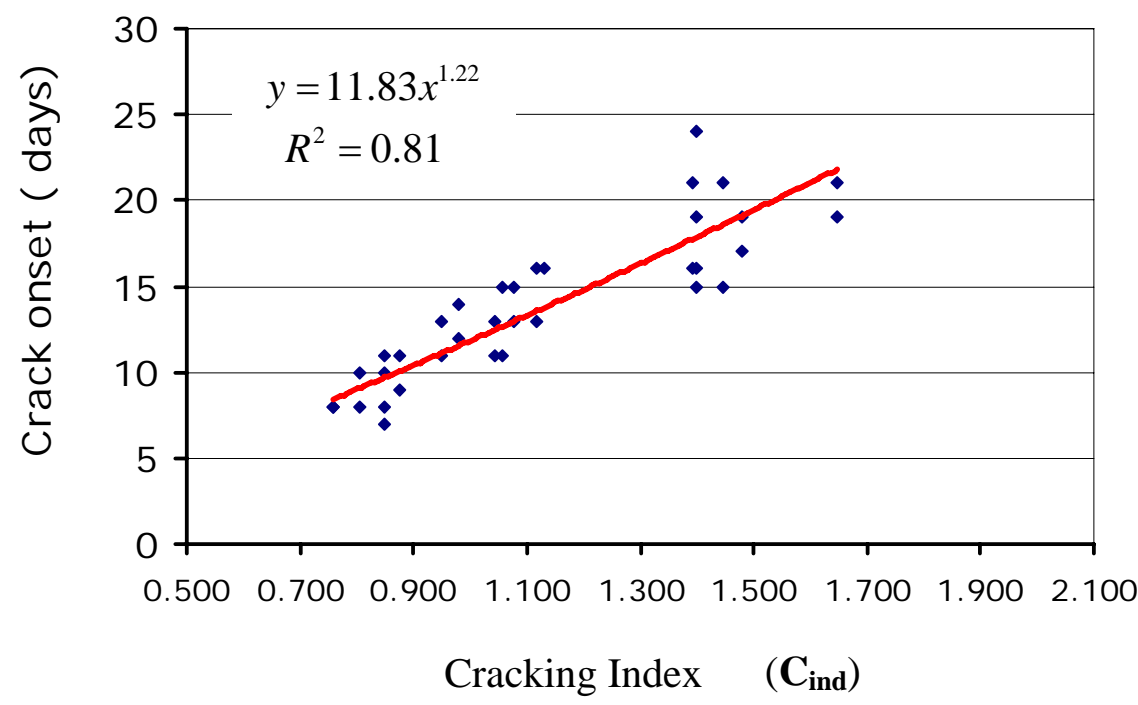

Figure 5.9 Regression analysis of cracking onset day and basic material parameters

The $X$-coordinate in Figure 5.9 can be expressed as

$$
C_{\text {ind }}=\frac{100 \times f_{c}^{\prime \frac{1}{10}}}{\varepsilon_{\text {free }} E^{1.2}}
$$

where: $f_{c}$ is 28-day compressive strength in standard curing condition,

$\varepsilon_{\text {free }}$ is 90-day free shrinkage,

$E$ is modulus of elasticity at 28 days.

$C_{i n d}$ is defined as the cracking index. In this definition, $f_{c}^{1 / 10}$ is taken as the strength property of HPC mixtures. The higher the compressive strength, the higher is the potential cracking resistance capacity within the range of compressive strength studied. The free shrinkage and modulus of elasticity influence the cracking resistance of HPC negatively, since the higher the values of those parameters, the greater the potential for cracking is. 
The prediction equation can then be expressed as:

$$
t=11.83 \cdot\left(C_{\text {ind }}\right)^{1.22}
$$

where: $t$ is predicted onset of cracking in days; $C_{i n d}$ is cracking index as expressed in Eq. (5.5).

Therefore, the above relationships allow predicting the onset of restraint cracking of a concrete with given strength, modulus of elasticity and free shrinkage. Table 5.3 shows the predicted cracking onset by using the Eq. (5.5) and Eq. (5.6). A good agreement with the laboratory measured data can be found.

Considering real-life data such as compressive strength and free shrinkage of concrete mixtures from well performing bridge decks in WV, the cracking index can readily be estimated. The cracking onset data corresponding to this crack index determined on practical data may be used as threshold value for design.

Table 5.4 lists part of field data of 28-day compressive strength and 90-day free shrinkage of bridge deck concrete mixtures from two projects in WV: 40th street in Charleston and I-64 in Dunbar, which had shown no concrete deck cracking for 3 years. The mixtures were made with gravel from local sources of WV. Since the data of modulus of elasticity were not available from these two projects, the equation (Eq. 4.3) derived in Chapter Four was used to estimate 28-day modulus of elasticity.

Table 5.4 also lists the cracking onset day calculated with Eq. (5.5) and Eq. (5.6) on the basis of these field data. It is shown that the predicted onset of cracking was $26 \pm 3$ days. It may be said that for any acceptable mix design of HPC made with gravel, the concrete must have cracking onset more than $26 \pm 3$ days, as shown in Figure 5.10. 
Table 5.4 Field data from $40^{\text {th }}$ Street and I-64 Dunbar projects

\begin{tabular}{|c|c|c|c|c|c|c|c|c|c|}
\hline \multirow{2}{*}{$\begin{array}{l}\text { Project } \\
\text { Name }\end{array}$} & \multicolumn{4}{|c|}{$\begin{array}{l}\text { 28-day comp. strength } \\
\text { (MPa) }\end{array}$} & \multicolumn{4}{|c|}{$\begin{array}{l}\text { 90-day free shrinkage } \\
(\mu \varepsilon)\end{array}$} & \multirow{2}{*}{$\begin{array}{c}\text { Cracking } \\
\text { onset } \\
\text { (days) }\end{array}$} \\
\hline & \#1 & \#2 & \#3 & Ave. & \#1 & \#2 & \#3 & Ave. & \\
\hline \multirow{14}{*}{$\begin{array}{c}40^{\text {th }} \\
\text { Street }\end{array}$} & 41.4 & 42.4 & 41.1 & 41.6 & 477 & 372 & 407 & 419 & 27 \\
\hline & 37.6 & 36.6 & 37.2 & 37.1 & 419 & 442 & 419 & 426 & 28 \\
\hline & 50.5 & 51.6 & 53.5 & 51.9 & 430 & 360 & 523 & 438 & 23 \\
\hline & 48.0 & 48.5 & 47.4 & 48.0 & 442 & 407 & 360 & 403 & 26 \\
\hline & 45.8 & 46.1 & 46.3 & 46.1 & 419 & 395 & 302 & 372 & 30 \\
\hline & 52.0 & 52.1 & 50.3 & 51.4 & 477 & 407 & 372 & 419 & 24 \\
\hline & 44.4 & 45.5 & 45.2 & 45.0 & 442 & 512 & 360 & 438 & 25 \\
\hline & 44.2 & 42.1 & 41.1 & 42.5 & 477 & 430 & 640 & 516 & 21 \\
\hline & 37.3 & 38.2 & 37.5 & 37.7 & 500 & 407 & 430 & 446 & 27 \\
\hline & 30.7 & 34.2 & 33.0 & 32.6 & 442 & 419 & 570 & 477 & 26 \\
\hline & 42.2 & 43.5 & 42.4 & 42.7 & 407 & 453 & 430 & 430 & 26 \\
\hline & 49.7 & 50.2 & 48.2 & 49.3 & 395 & 477 & 442 & 438 & 23 \\
\hline & 43.7 & 41.1 & 42.8 & 42.6 & 395 & 372 & 395 & 388 & 29 \\
\hline & 43.7 & 44.0 & 43.3 & 43.7 & 535 & 535 & 500 & 523 & 20 \\
\hline \multirow{15}{*}{$\begin{array}{c}\text { I-64 } \\
\text { Dunbar }\end{array}$} & 52.6 & 54.0 & 51.9 & 52.8 & 337 & 465 & 349 & 384 & 26 \\
\hline & 50.6 & 48.7 & 51.6 & 50.3 & 453 & 360 & 407 & 407 & 25 \\
\hline & 45.1 & 44.7 & 43.1 & 44.3 & 360 & 337 & 651 & 450 & 24 \\
\hline & 48.2 & 47.3 & 46.4 & 47.3 & 570 & 372 & 372 & 438 & 24 \\
\hline & 46.3 & 45.9 & 46.1 & 46.1 & 407 & 453 & 256 & 372 & 30 \\
\hline & 47.6 & 45.8 & 48.9 & 47.4 & 407 & 337 & 419 & 388 & 28 \\
\hline & 52.2 & 50.2 & 50.6 & 51.0 & 360 & 395 & 488 & 415 & 25 \\
\hline & 51.0 & 51.9 & 50.6 & 51.2 & 500 & 314 & 419 & 411 & 25 \\
\hline & 40.0 & 39.7 & 42.4 & 407 & 500 & 372 & 465 & 446 & 25 \\
\hline & 47.0 & 47.7 & 47.3 & 47.4 & 279 & 419 & 419 & 372 & 29 \\
\hline & 49.9 & 51.5 & 50.0 & 50.5 & 349 & 384 & 384 & 372 & 28 \\
\hline & 50.0 & 51.1 & 49.9 & 50.3 & 360 & 372 & 384 & 372 & 28 \\
\hline & 46.4 & 48.1 & 48.9 & 47.8 & 384 & 384 & 360 & 376 & 29 \\
\hline & 50.5 & 52.6 & 50.3 & 51.2 & 407 & 407 & 430 & 415 & 24 \\
\hline & 51.5 & 51.5 & 52.2 & 51.7 & 500 & 407 & 360 & 422 & 24 \\
\hline
\end{tabular}




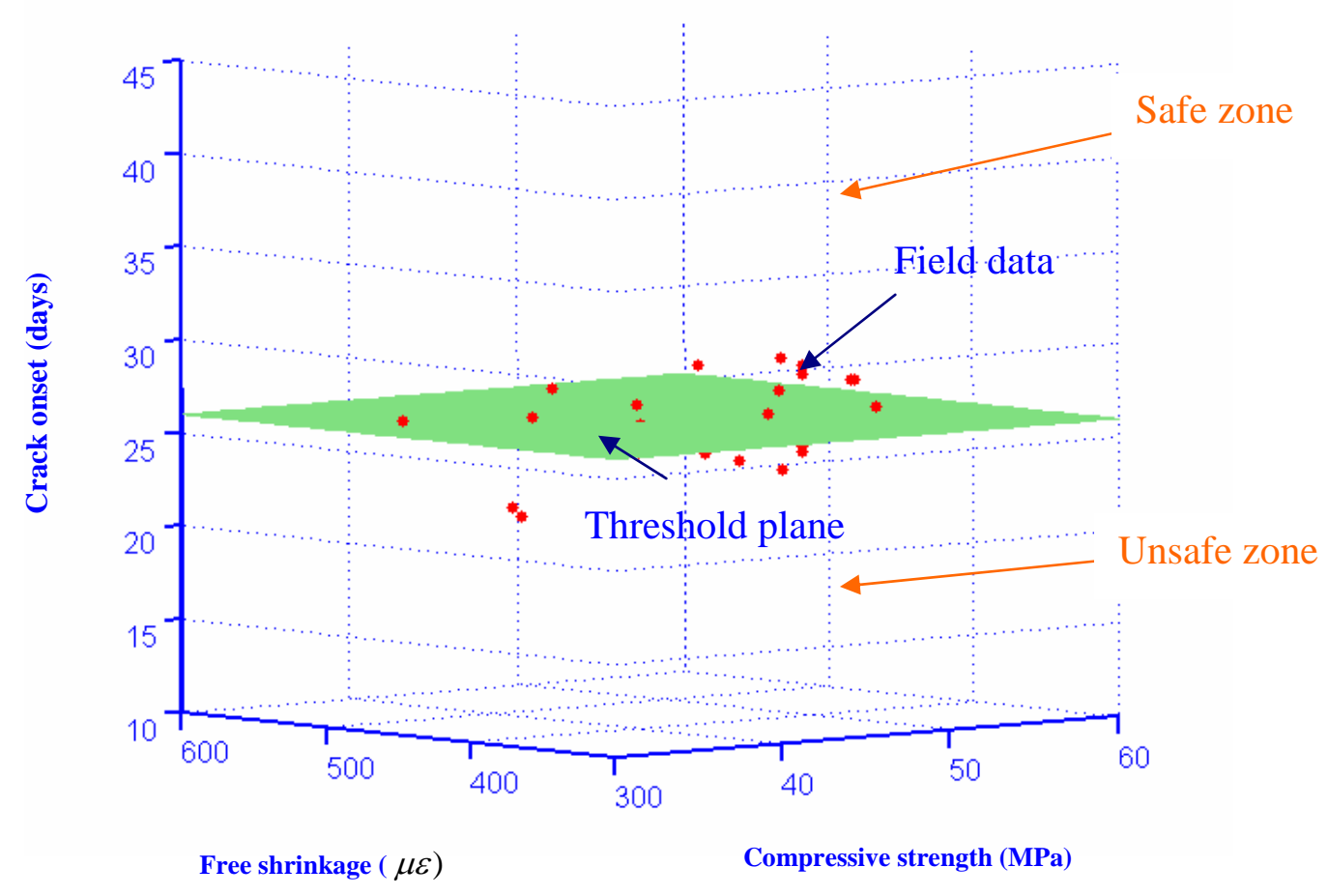

Figure 5.10 Threshold plane of cracking onset based on field data

In order to obtain a more accurate threshold number, more field data from WVDOH are needed. For improving the relationship of cracking index and cracking onset, further research may be undertaken with more types of aggregates. The threshold number $26 \pm 3$ days mentioned above is good for gravel HPC; however, for limestone HPC, the threshold number is expected to be less. 


\subsection{Concluding Remarks}

1. The AASHTO ring together with strain gauges can capture the cracking onset with reasonable accuracy, and is an effective way to evaluate the cracking properties of different HPC mixtures.

2. The elastic analysis of ring specimen showed that at cracking onset, the maximum stress inside the concrete ring is lower than the tensile strength of the concrete material. This indicates that the conventional strength method is not suitable for the failure analysis of ring specimen.

3. HPC with gravel generally cracked 2 8 days later than HPC with limestone under the same conditions. For HPC with lower w/cm, the crack occurred earlier. For HPC with different SCM combinations, only slight differences were found on the cracking onset among the specimens.

4. A simplified approach (so called cracking index) derived from most common material parameters such as compressive strength, modulus of elasticity, and free shrinkage is recommended to evaluate cracking properties of different HPC mixtures.

5. Real-life data such as compressive strength and free shrinkage of HPC mixtures from well performing bridge decks within WV were collected, and the cracking index were calculated. A threshold cracking onset day is proposed as the criterion for any acceptable mix design of HPC with gravel. Suggestions are made for HPC with limestone.

6. In order to obtain a more reliable expression for cracking index, more experimental work is needed. More real life field data are also required to establish the threshold number for gravel HPC as well as limestone HPC. 


\section{CHAPTER SIX}

\section{NUMERICAL ANALYSIS OF CRACKING OF RING SPECIMENS}

\subsection{Introduction}

The conventional analysis method of concrete structure is based on failure criteria which assume that once the induced stress reaches the ultimate limit, the structure fails. However, as it was concluded in previous chapters, the tensile strength of concrete is about $1 / 20$ th of its compressive strength, and the failure of most concrete structures is initiated by the propagation of tensile cracks, thus the failure process should be described in terms of energy rather than strength. Fracture mechanics is believed to be an effective method for analyzing concrete structures. The reasons for advantages of fracture mechanics over the conventional methods will be discussed in Chapter Seven.

In this chapter, a numerical analysis based on the concept of fracture mechanics is developed to predict the cracking onset of the ring specimens. The numerical results are compared with the laboratory test data. In this numerical procedure, the modulus of elasticity, free shrinkage, and the fracture mechanics test results are adopted as the important input parameters. The purpose of this analysis is to validate the effectiveness of the AASHTO ring specimen test, which is the only recommended test used for evaluating cracking tendency of concrete. 


\subsection{Failure Criteria Based on Fracture Mechanics}

Fracture mechanics is the theory which governs initiation and propagation of cracks. The microcracks which exist in concrete structures intensify local stress distribution. If the energy stored at vicinity of a crack tip is equal to the energy required for the formation of new surfaces, then crack growth can take place. According to Griffith energy principle (Griffith, 1920) as discussed in Chapters Two, the crack extension occurs when the condition as described in Eq. (2.1) is satisfied. Equation (2.1) can be rewritten as:

$$
\frac{\partial(W-U)}{\partial a}=\frac{\partial \Gamma}{\partial a}
$$

considering a plane structure of a unit thickness, Eq. (6.1) becomes:

$$
G=R
$$

where:

$$
\begin{aligned}
& G=\frac{\partial(W-U)}{\partial a} \text { is the energy release rate; } \\
& R=\frac{\partial \Gamma}{\partial a} \text { is the material resistance to crack extension. }
\end{aligned}
$$

A plot of $G$ versus crack extension $\Delta a$ is called the driving force curve or G-curve, and a plot of $\mathrm{R}$ versus crack extension $\Delta a$ is called resistance curve or R-curve. The concept of R-curve was first introduced by Irwin (McCabe, 1973) for the study of metals based on energy criterion. The R-curve approach has been widely used for fracture of quasi-brittle materials like ceramics and concrete. Wecharatana and Shah (1983) used an R-curve to model the stable crack propagation of concrete. Bazant et al. (1986) proposed an R-Curve method from the size effect law. Quyang and Shah (1991) derived a simple 
formulation of R-curve by approximating the G-curve with a second order function of the crack extension length. In their approach, the R-curve was defined as an envelope of different G-curves obtained by varying the dimensions of identical specimens. Elices and Planas (1993) introduced the concept of equivalence method to study the difference that is caused by a certain elastic equivalence between cohesive materials and linear elastic materials. The R curves of different geometric specimens were developed and used to predict the behavior of cohesive materials like concrete. Shah et al. (1998) proposed a prediction model for the cracking of the ring specimen by using the R-curve concept based on the two parameter method. Lou et al. (2003) proposed a numerical model of Rcurves of different concrete by utilizing the weight function in the estimation of the weighted distributions of the cohesive force across the fracture process zone. The predicted R-curve behavior was compared with the measured R-curve.

The R-curve for a perfect brittle material is flat because the surface energy is a constant material property. However, for nonlinear material accompanying fracture, the R-curve shows a variety of shapes. For instance, the R-curve of concrete is a rising function with crack extension length due to the nonlinear behavior of the fracture process zone ahead of the crack. Ideally, the fracture resistance R should be a material property and independent of the size and shape of the structural body. However, the geometry of the cracked structures can exert some influence on the shape of the R-curve.

Generally, the energy release rate $G$ is a function of applied stress, and the fracture resistance curve R usually is an unknown function of crack extension length. Figure 6.1 shows a typical R-curve configuration of concrete and G-curves at different stress levels. 


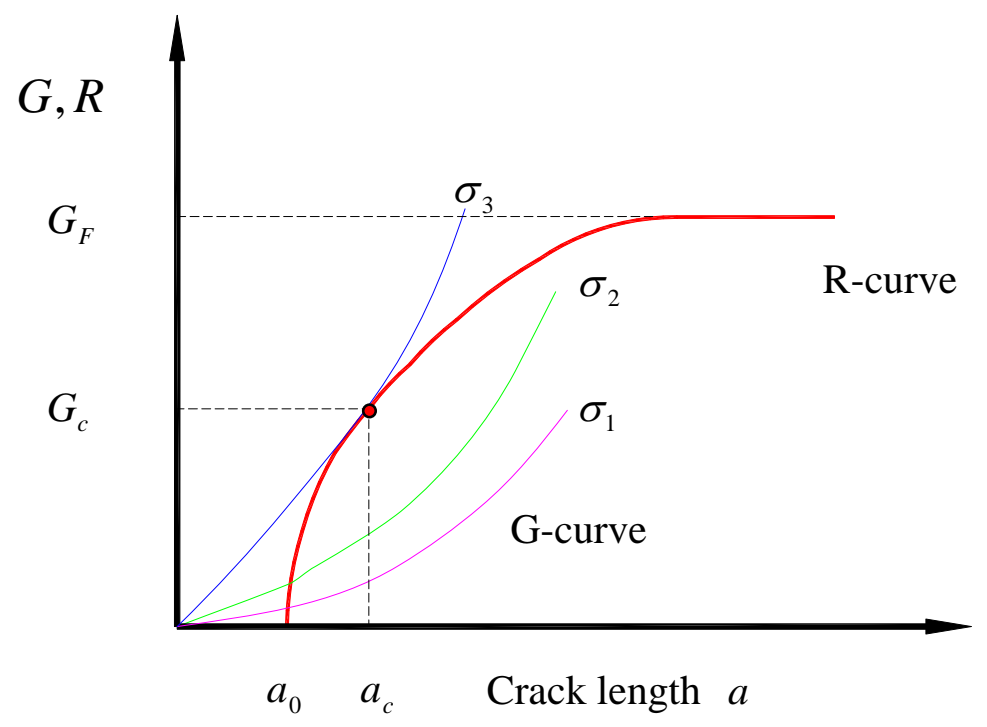

Figure 6.1 Failure criteria based on fracture mechanics

In a quasi-static fracture process, Eq. (6.2) is satisfied whenever the crack is growing. The crack propagation may be stable or unstable. According to the definition of R-curve, the following condition should be satisfied at the critical crack propagation:

$$
\left(\frac{\partial G}{\partial a}\right)_{c}=\left(\frac{\partial R}{\partial a}\right)_{c}
$$

Both Eq. (6.2) and Eq. (6.3) provide the necessary and sufficient conditions for failure of concrete structures based on fracture mechanics. As shown in Figure 6.1, the critical point is defined in the $R, G-a$ plots by the condition that the G-curve be tangent to Rcurve.

According to the above descriptions, the procedure to determine the failure of a concrete structure can be summarized as follows: 
1. Define or construct the R-curve, which is regarded as a material property and independent of the geometry under certain conditions. The best way to find the Rcurve for certain concrete material is by fracture mechanics testing.

2. Derive the G-curve for the specific structure. A numerical method is necessary to obtain the G-curve for a structure.

3. Solve Eq. (6.3) analytically or numerically and find the critical condition.

\subsection{G-Curves for AASHTO Concrete Ring}

A variety of numerical techniques have been employed to solve the problem from fracture mechanics. Among those numerical techniques, the boundary element method (BEM) has become an emerging numerical technique, which provides an efficient alternative to finite difference or finite element methods for solving certain types of fracture problems. Shah (1998) employed BEM method to numerically compute the stress intensity factor for the ring specimens with a size different from the AASHTO ring. However, the lack of availability of commercial software using BEM technique made it difficult to implement the application of this method. In this study, the well-established FE analysis software ANSYS was used to derive the G-curves for the AASHTO ring specimen.

As it was discussed in Chapter Five, the concrete ring was restrained against horizontal movement by the steel ring. When the concrete shrank, the circumferential stress developed, and the concrete rings behaved as a tension member. Cracks were initiated from the inner boundary, as shown in Figure 6.2 (a). When $r_{2}$ approaches 
infinity and $\frac{r_{1}}{r_{2}}$ remains constant, the ring specimen becomes an infinitely large plate, as shown in Figure 6.2 (b)

According to the fracture mechanics theory (Tada et al., 2000), for an infinitely large plate under uniaxial tension, the stress intensity factor can be calculated by:

$$
K=1.122 \sigma \sqrt{\pi a}
$$

For the ring specimen, the stress intensity factor can then be calculated by

$$
K=1.122 \sigma_{\theta \theta} \sqrt{\pi a} F(a / b)
$$

where $\sigma_{\theta \theta}$ is circumferential stress built inside the concrete ring; $a$ is the crack extension length; $b=r_{1}-r_{2}$; and $F\left(\frac{a}{b}\right)$ is the geometrical function which is dependent on the size of the ring specimen.

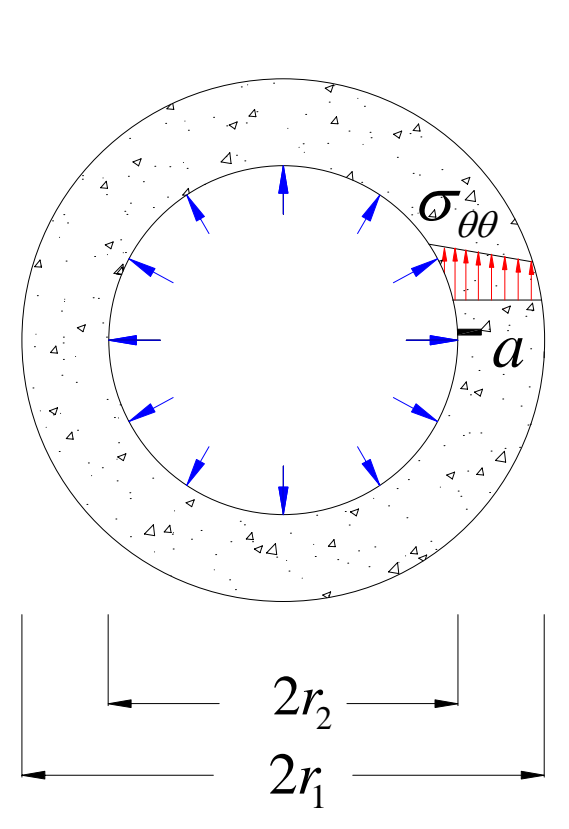

(a)

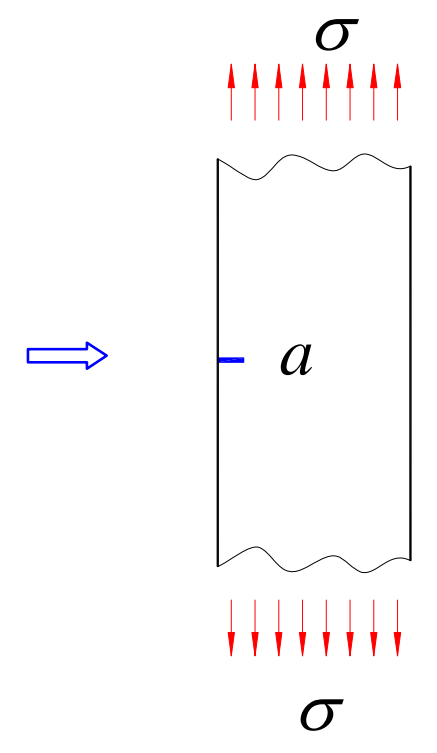

(b)

Figure 6.2 Simplification of ring specimen for fracture analysis 
In order to find the geometrical function $F\left(\frac{a}{b}\right)$, a finite element analysis was conducted to calculate the stress intensity factors of the concrete rings with different initial crack lengths such as:

$a_{0}=0.03 b, a_{0}=0.06 b, a_{0}=0.1 b, a_{0}=0.2 b, a_{0}=0.3 b, a_{0}=0.4 b, a_{0}=0.5 b$,

$a_{0}=0.6 b, a_{0}=0.7 b, a_{0}=0.8 b$, and $a_{0}=0.9 b$.

Due to the geometrical symmetry of the ring specimen, only one half of the ring was modeled. Figure 6.3 shows the 2-D mesh for the FE model of the ring specimen. A unit pressure was applied to the interior surface to simulate the restraining force from the steel ring.

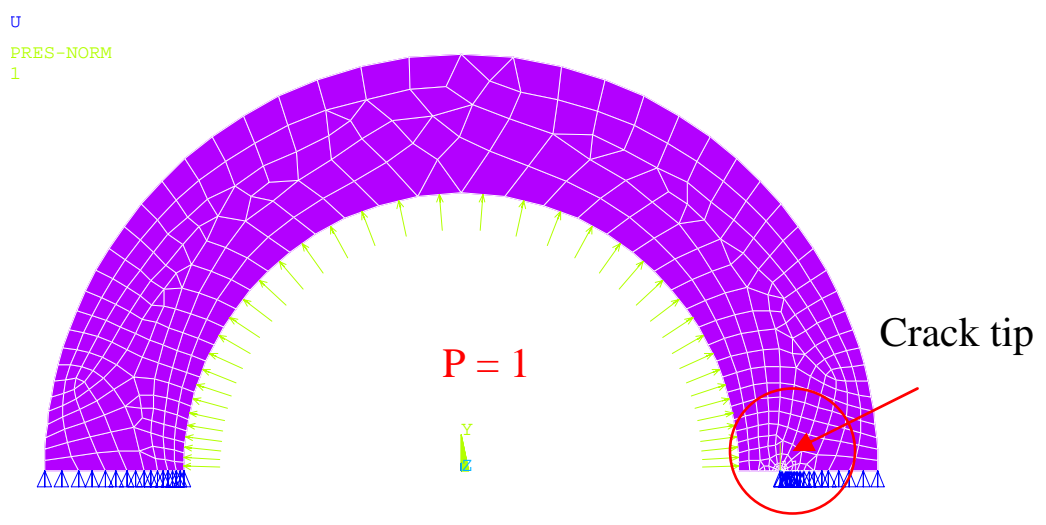

(a)

Deformed crack

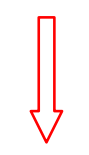

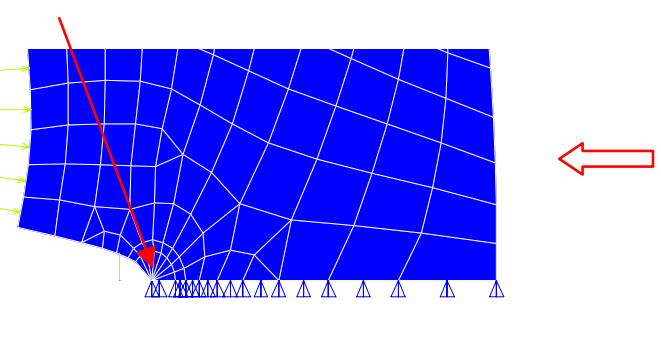

(c)

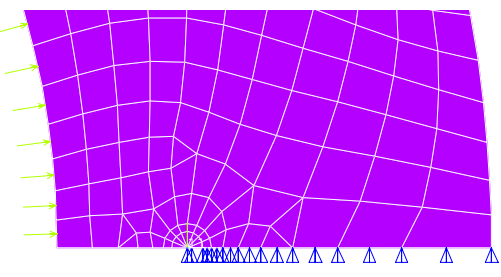

(b)

Figure 6.3 2-D mesh for FE model of ring specimen 
As stated in the ANSYS manual (ANSYS, 2005), the most important region in a fracture model is the region around the edge of the crack. The first row of elements around the crack tip should be singular. The element type adopted in this study was PLANE2, the 6-node triangular solid, as shown in Figure 6.4, which is recommended by ANSYS for the 2-D fracture analysis.

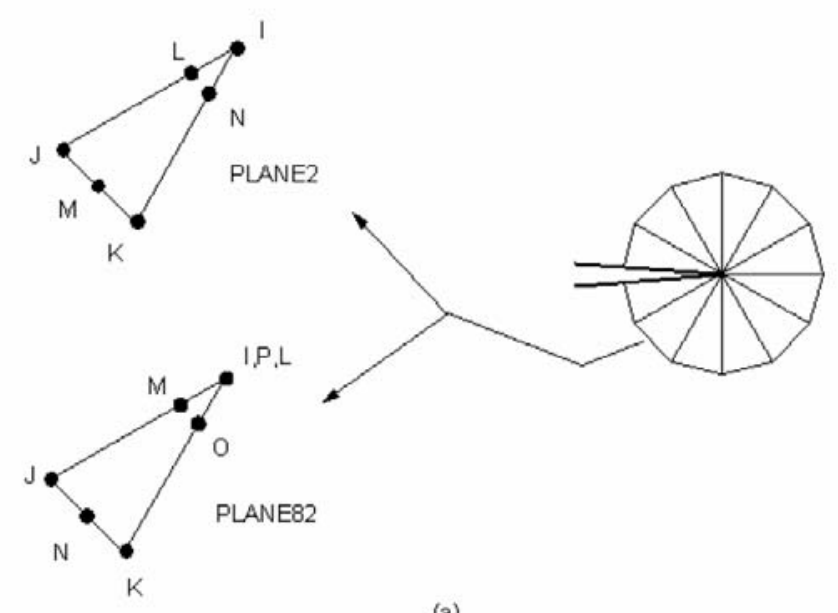

(a)

Figure 6.4 Element type for fracture mechanics analysis

(From: ANSYS, 2005)

Thus, for each initial crack length $a_{0}\left(a=a_{0}\right)$, the corresponding stress intensity factor $K$ can be calculated through FE analysis. According to Eq. (6.5), the $F\left(\frac{a}{b}\right)$ value can further be calculated. A plot of $F\left(\frac{a}{b}\right)$ versus $\frac{a}{b}$ is shown in Figure 6.5. Based on best fitting analysis, the $F\left(\frac{a}{b}\right)$ can be expressed as: 


$$
F\left(\frac{a}{b}\right)=1.655-2.437\left(\frac{a}{b}\right)+10.531\left(\frac{a}{b}\right)^{2}-11.431\left(\frac{a}{b}\right)^{3}+5.244\left(\frac{a}{b}\right)^{4}
$$

for the ring specimen with external radius $r_{1}=228 \mathrm{~mm}$, and internal radius $r_{2}=152 \mathrm{~mm}$, and $b=76 \mathrm{~mm}$.

From Eq. (5.3 a) and Eq. (5.4), the expression for Eq. (6.5) can be rewritten as:

$$
K=1.122 \beta E \varepsilon_{\theta \theta} \sqrt{\pi a} F(a / b)
$$

where:

$$
\beta=\frac{\left(r_{1}^{2} r_{2}^{2} / r^{2}+r_{2}^{2}\right)}{r_{1}^{2}+r_{2}^{2}+\mu\left(r_{1}^{2}-r_{2}^{2}\right)}
$$

$r_{1}, r_{2}$ are external and internal radii, respectively; $r$ is the radius at the point where the stress is calculated; $\mu$ is Poison's ratio; $\varepsilon_{\theta \theta}$ is circumferential strain caused by restrained shrinkage; $F\left(\frac{a}{b}\right)$ can be calculated by Eq. (6.6), and E is the modulus of elasticity of concrete. In this study, the chord tensile modulus of elasticity obtained by direct tensile test at failure was used.

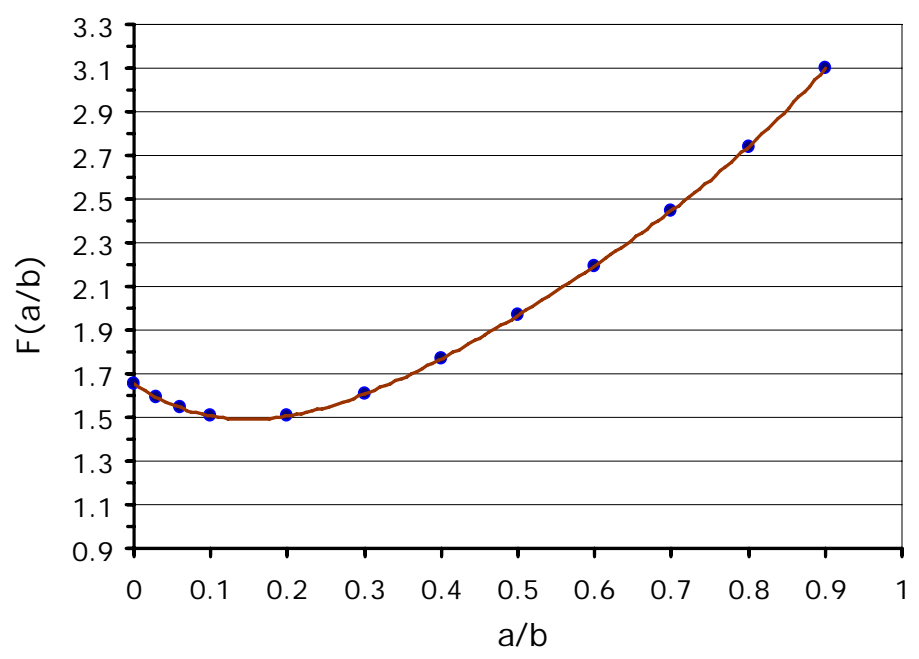

Figure 6.5 The geometry function for ring specimen 
It is to be noted that the stress along the cross section of the ring specimen is not uniformly distributed. In this study, the stress at the middle section of the ring was used. By assuming the Poisson's ratio $\mu=0.2$, and substituting $r_{1}=228, r_{2}=152$ and $r=190$ (middle of the cross section) into Eq. (6.8), the value of $\beta$ became 0.69. The Gcurve of the AASHTO ring specimen can be expressed as:

$$
G=\frac{K^{2}}{E}=0.6 \pi E \varepsilon_{\theta \theta}^{2} a F(a / b)^{2}
$$

Figure 6.6 presents the typical G-curves for concrete rings with modulus of elasticity $E=28630 \mathrm{MPa}$, and three different strains corresponding to three different stress levels: $\varepsilon_{\theta \theta}=20 \mu \varepsilon, \varepsilon_{\theta \theta}=80 \mu \varepsilon$, and $\varepsilon_{\theta \theta}=120 \mu \varepsilon$ respectively.

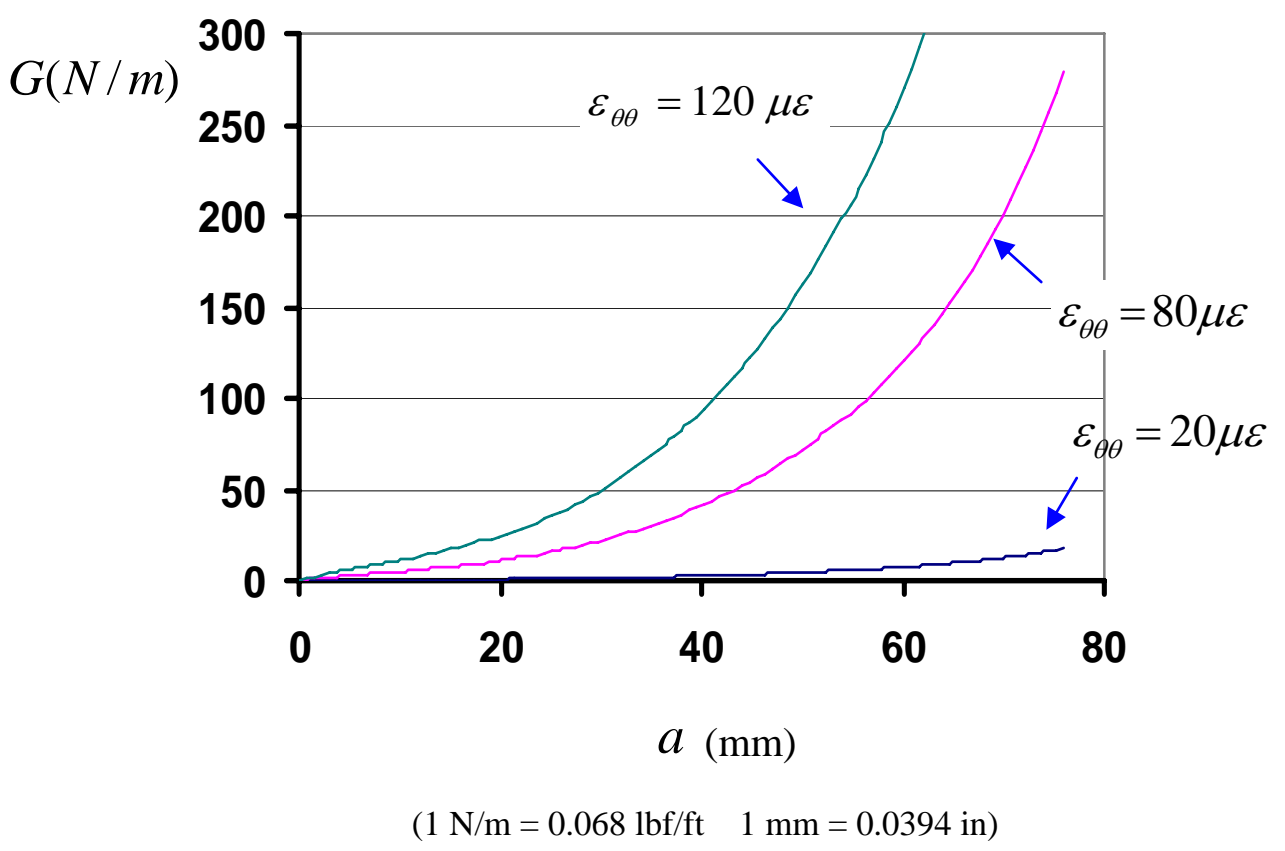

Figure 6.6 Typical G-curves for AASHTO ring at different stress levels 


\subsection{R-Curves for Different HPC Mixtures}

The construction of the R-curves for different HPC mixtures was based on the concept of equivalent elastic crack model, which was first developed by Elices and Planas (1993).
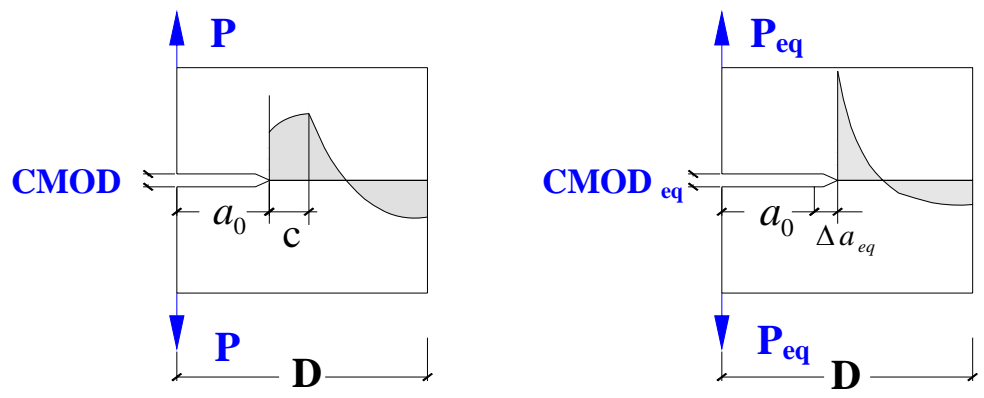

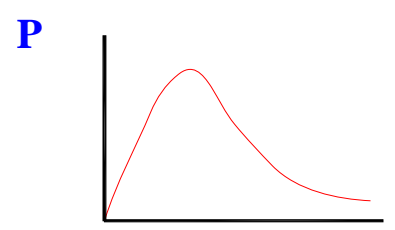

CMOD

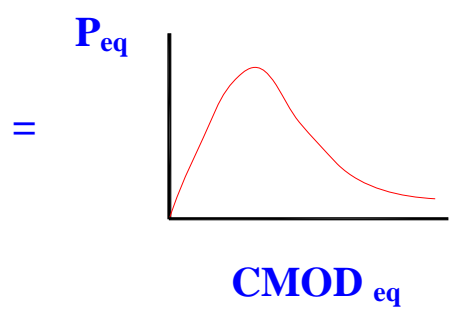

Figure 6.7 Definition of the P-CMOD equivalence

(Adopted from: Elices and Planas, 1993)

Concrete being a quasi-brittle material, the LEFM procedures can not be directly applied to solve the fracture related problems. The equivalent elastic crack approach emerges as an intermediate technique to avoid cumbersome computing while using the simple LEFM procedures with an associate R-curve.

As shown in Figure 6.7, the actual sample is on the left side, and is made of a cohesive material such as concrete. Its cohesive zone grows monotonically up to $c$ with corresponding load P. On the right side, it is the so called equivalent sample, which is 
made of elastic material. Both samples are geometrically identical. The measured PCMOD (crack mouth opening displacement) responses of these two samples are different. However, the P-CMOD responses can purposely be forced to match each other by choosing a suitable equivalent crack length $a_{e q}$, with $a_{e q}=a_{0}+\Delta a_{e q}$, as shown in Figure 6.7. The LEFM procedure can then be used to obtain the R- $\Delta a$ curves. However, the Rcurve constructed in this way is not a material property but depends on the geometry and size of the specimen. Further studies by Elices and Planas (1993) concluded that if the length of the relative ligament size is similar to members with different geometry or size, the $\mathrm{R}$ curve constructed can be regarded as a material property.

As concluded in Chapter Five, the cracking onset of limestone HPC occurred earlier than that of gravel HPC. Also for HPC mixtures with different w/cm, significant differences were found for cracking onset days. Therefore, three different HPC mixtures (SL-SF-L1-0.4, SL-SF-G2-0.4, and SL-SF-L1-0.3), which could represent these differences, were selected for numerical analysis. The three-point bending fracture mechanics test method was employed to obtain the P-CMOD responses. The detailed description of the test setup and testing procedure of the three-point bending fracture mechanics test are given in the next chapter. The sketch of the specimen is shown in Figure 6.8 (a). A $10 \mathrm{~mm}$ (0.4 in) long notch was cut at the mid-span of the beam. The length of the beam was $483 \mathrm{~mm}$ (19 in) and the span to depth ratio ( $S / b)$ was 4 . The ligament of the beam was around $90 \mathrm{~mm}$ (3.6 in) which is close to the thickness of the ring specimen $(76 \mathrm{~mm}=3 \mathrm{in})$. Figure 6.8 (b) shows a close view of the test setup. 


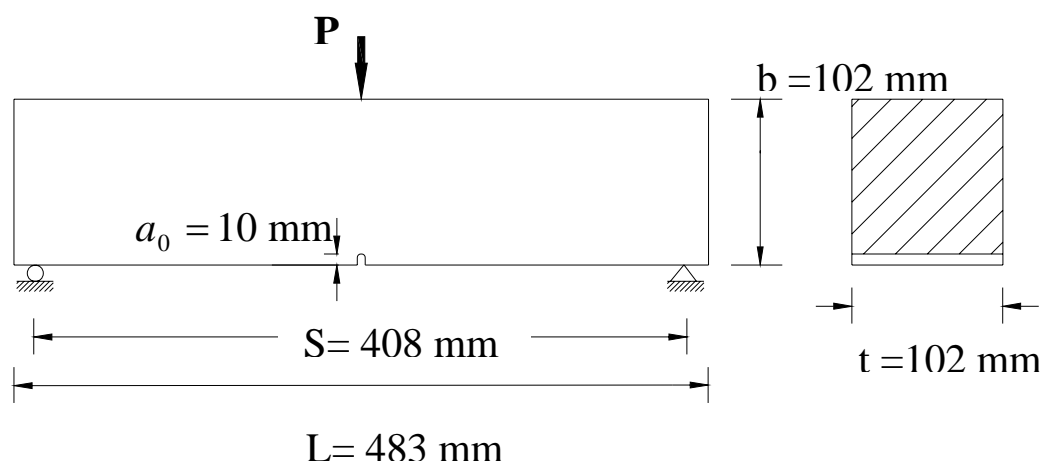

(a)

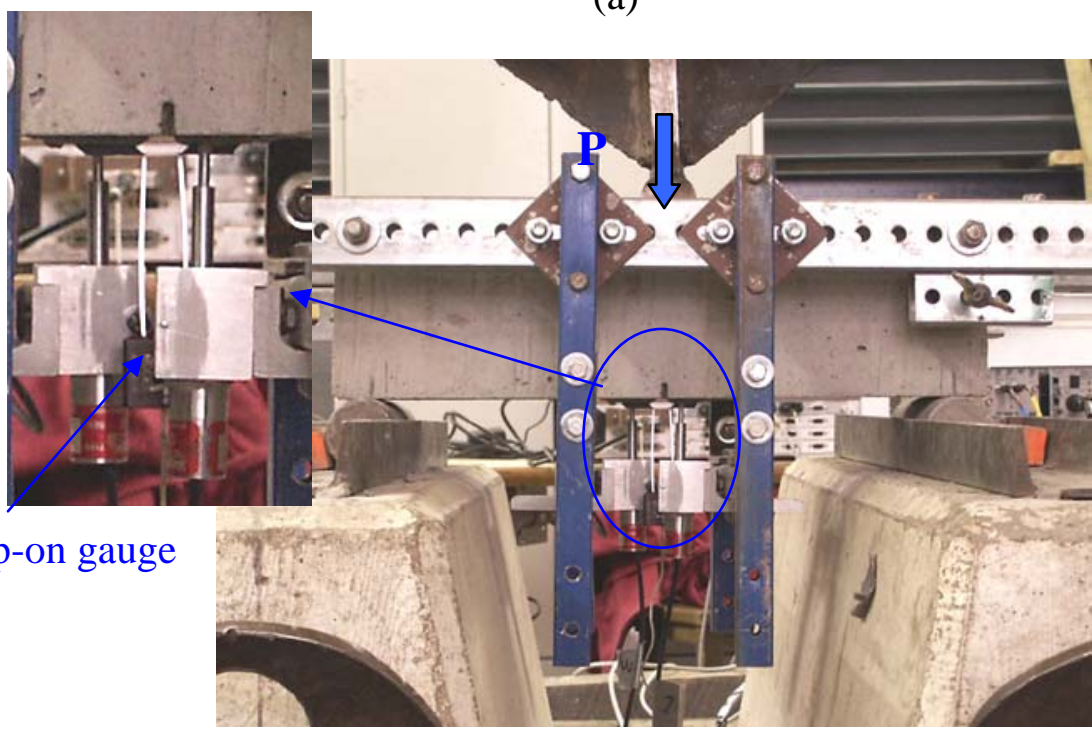

(b)

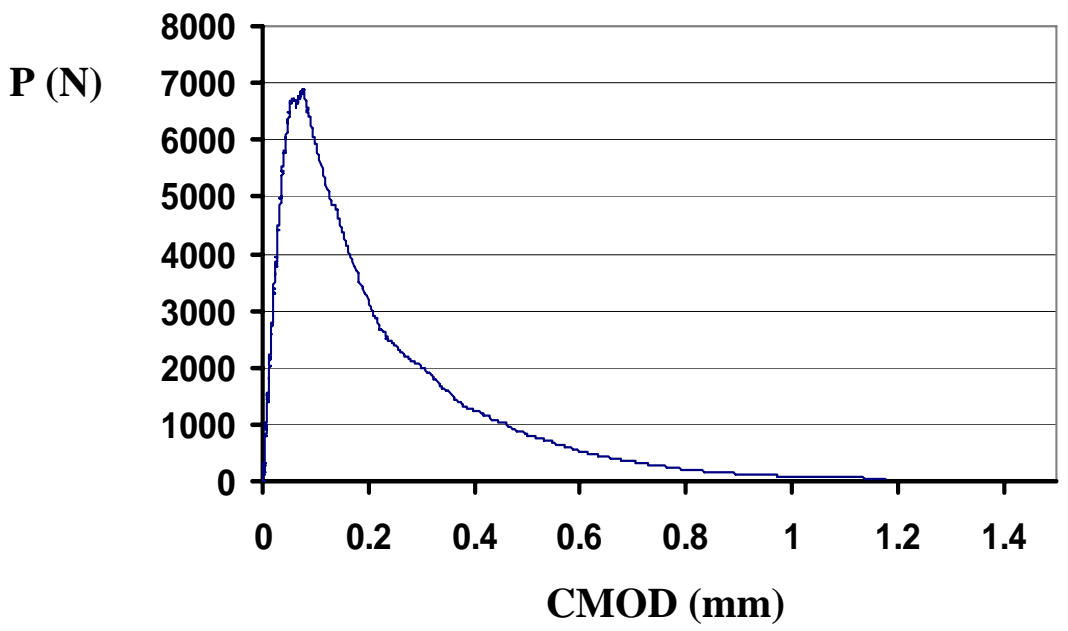

(c)

$(1 \mathrm{~mm}=0.0394$ in $1 \mathrm{~N}=0.224 \mathrm{lbf})$

Figure 6.8 Fracture mechanics test setup and typical P-CMOD response 
One clip-on gauge and two displacement transducers were mounted at the mid-span. The clip-on gauge with an accuracy of $0.005 \mathrm{~mm}$ (0.0002 in) was used to measure the PCMOD response. Two displacement transducers with an accuracy of $0.01 \mathrm{~mm}$ (0.0004 in) were used to measure the P-MSD (mid-span displacement) responses to calculate the fracture energy $G_{F}$ of different HPC (The method to calculate $G_{F}$ is also described in Chapter Seven). Figure 6.8 (c) provides a typical P-CMOD response obtained in this test.

When $\mathrm{P}$ and $C M O D$ are measured for the actual specimen, the equivalent elastic crack length $a_{e q}$ can be calculated from the linear elastic analysis:

$$
C_{e q}(a)=\frac{C M O D}{P}
$$

where $C_{e q}$ is the expression for the compliance associated with $C M O D$ for no-cohesive sample. According to the handbook of stress analysis of crack (Tada et al. 2000) for three-point bending test, the Eq. (6.10) can be expressed as:

$$
\frac{C M O D}{M}=\frac{24 a}{E b^{2} t} f_{1}(a / b)
$$

where $M$ is the moment at the mid-section, which can be expressed as:

$$
M=P S / 4+w L(2 S-L) / 8
$$

$f_{1}(a / b)$ is a geometry function, which can be expressed as:

$$
\begin{array}{r}
f_{1}(a / b)=0.76-2.28(a / b)+3.87(a / b)^{2}-2.04(a / b)^{3}+\frac{0.66}{(1-a / b)^{2}} \\
(\text { for } S / b=4)
\end{array}
$$

where $a$ is the crack length; $\mathrm{b}$ is the depth of the beam; $E$ is the modulus of elasticity; $S$ is the span of the beam; $L$ is the length of the beam; $t$ is the thickness of the beam, and $w$ is the unit weight of the beam. 
For every measured $C M O D$ and corresponding P value, from Eq. (6.11), Eq. (6.12), and Eq. (6.13), by solving a $6^{\text {th }}$ order polynomial function, the equivalent elastic crack length $a_{e q}\left(a_{e q}=a\right)$ can be obtained. After computing $a_{e q}$, the stress intensity factor can be calculated as:

$$
K=\frac{6 M \sqrt{a}}{b^{2} t} f_{2}(a / b)
$$

where $f_{2}(a / b)$ is a geometry function, which can be expressed as:

$$
f_{2}(a / b)=\frac{1.99-(a / b)(1-a / b)\left[2.15-3.93(a / b)+2.7(a / b)^{2}\right]}{[1+2(a / b)](1-a / b)^{3 / 2}}
$$

$$
(\text { for } S / b=4 \text { ) }
$$

The R-curve for HPC based on the P-CMOD test can be expressed as:

$$
R=\frac{K^{2}}{E}
$$

For every HPC mixture, the tests were conducted at the ages of 4, 7, 14, and 28-day. For each age, three replicate specimens were tested. Figure 6.9 (a), (b), (c) represent the P-CMOD responses at different ages for SL-SF-L1-0.4, SL-SF-G2-0.4, and SL-SF-L10.3 , respectively. The above mentioned numerical procedure was followed to construct R-curves from corresponding P-COMD responses. The numerical procedure was achieved by a computing program (Appendix D). Figure 6.10 (a), (b), (c) show the Rcurves at different ages for SL-SF-L1-0.4, SL-SF-G2-0.4, and SL-SF-L1-0.3, respectively. 


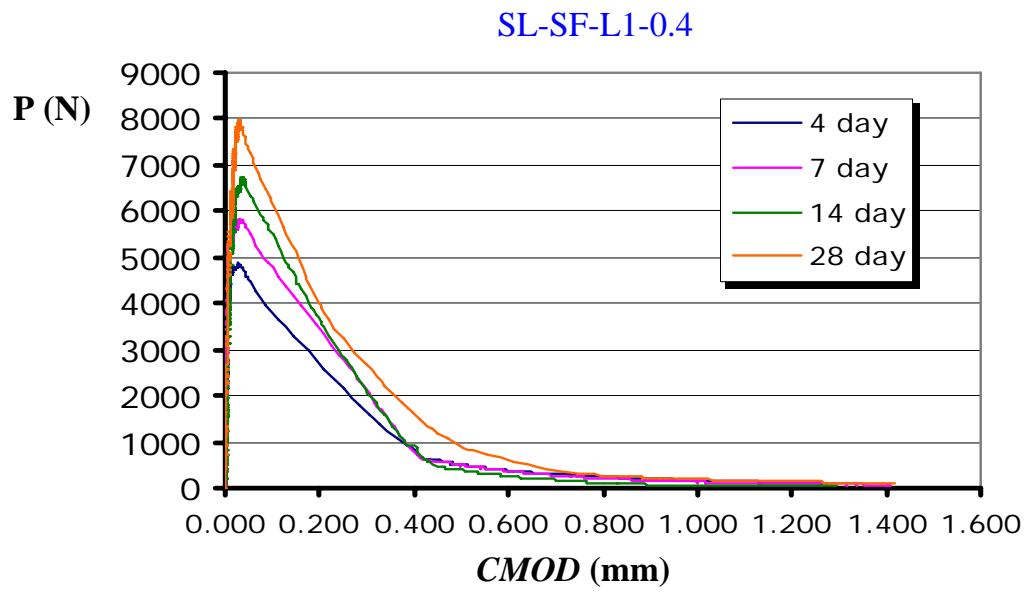

(a)

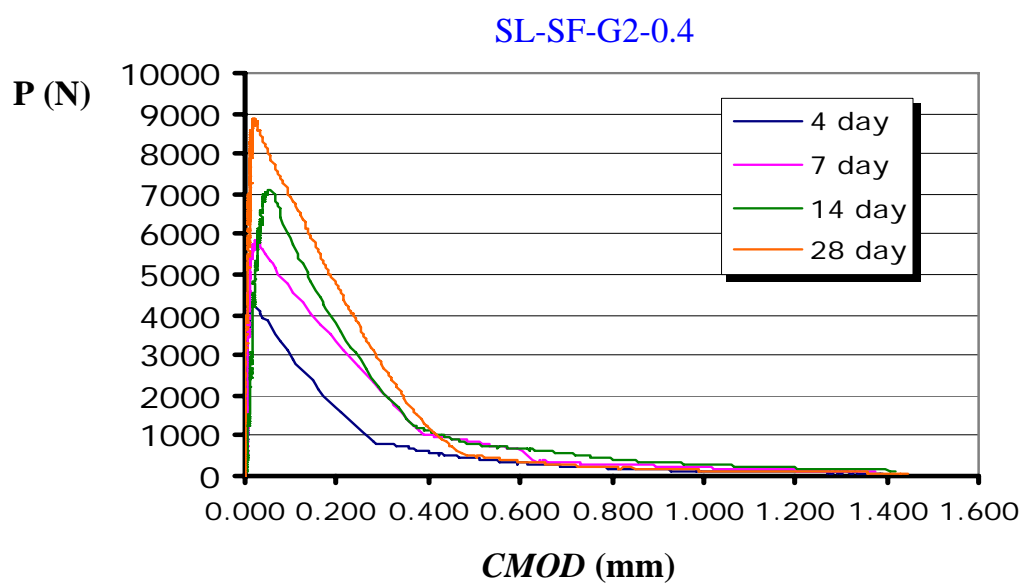

(b)

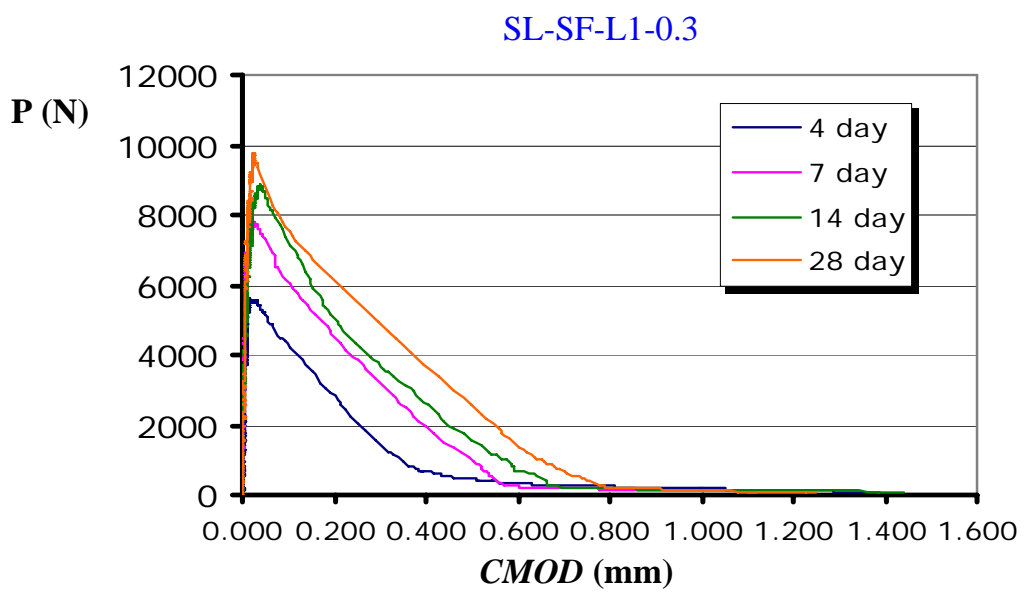

(c)

$(1 \mathrm{~mm}=0.0394$ in $1 \mathrm{~N}=0.224 \mathrm{lbf})$

Figure 6.9 The P-CMOD responses for HPC mixtures at different ages 


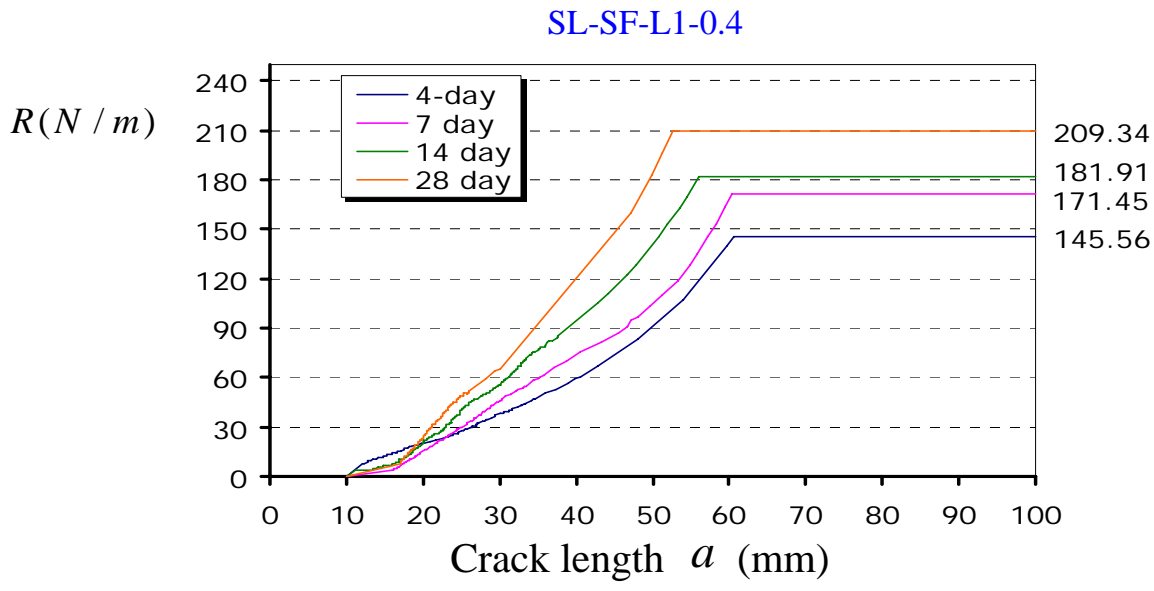

(a)

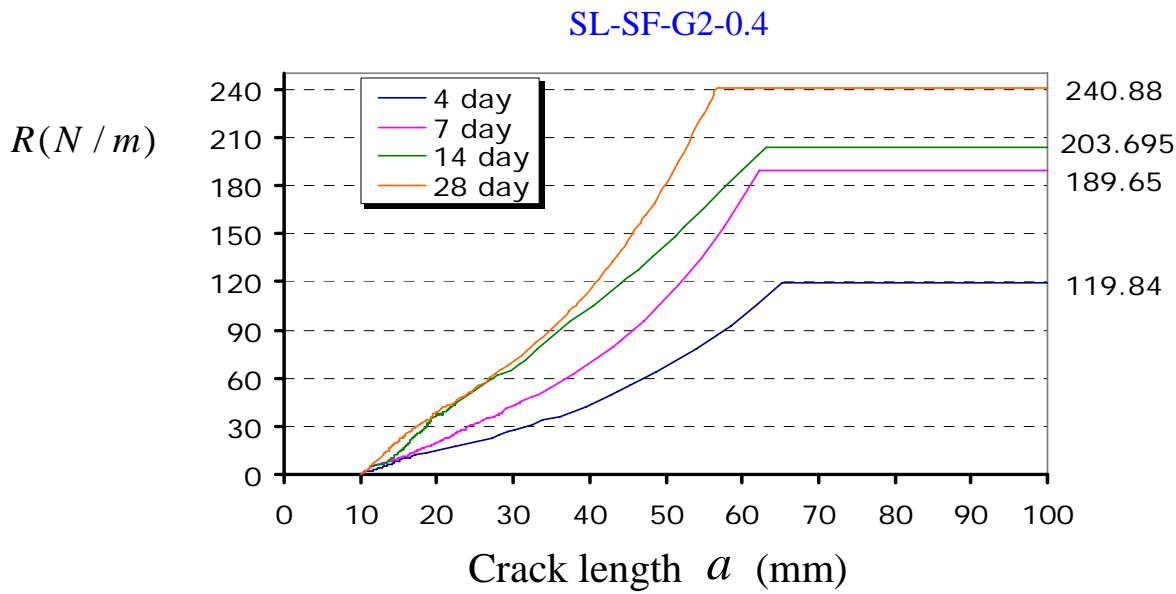

(b)

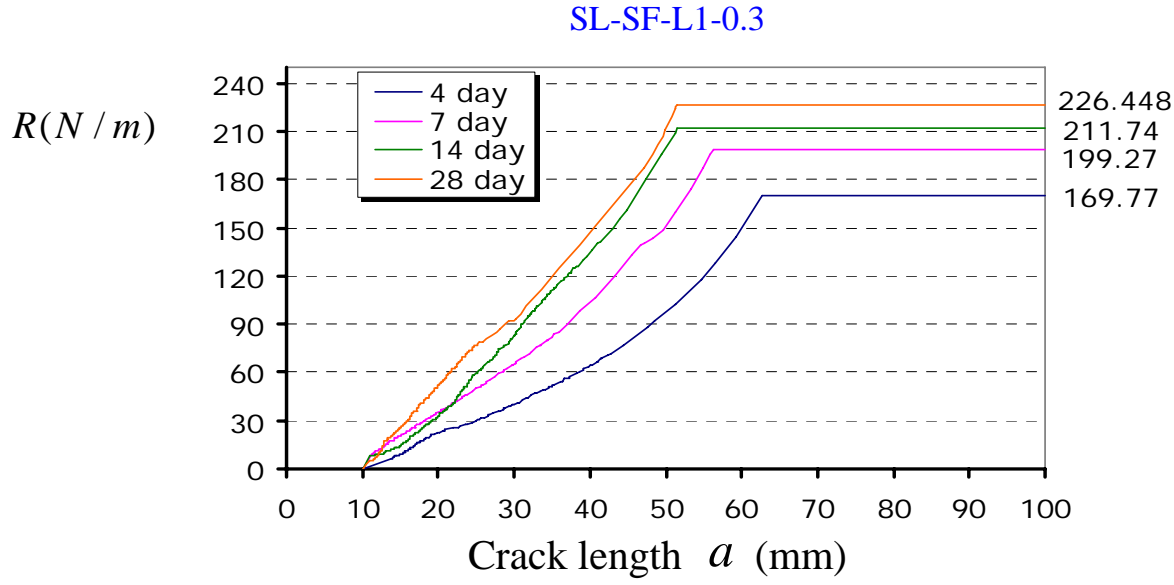

(c)

$(1 \mathrm{~N} / \mathrm{m}=0.068 \mathrm{lbf} / \mathrm{ft} \quad 1 \mathrm{~mm}=0.0394 \mathrm{in})$

Figure 6.10 The R-curves for HPC mixtures at different ages 


\subsection{Maximum Allowable Tensile Strains}

The above obtained G and R-curves can be substituted into Eq. (6.2) and Eq. (6.3) to determine the critical crack length $a_{c}$. To numerically solve those two equations, a computing program was developed as listed in Appendix E.

Figure 6.11, Figure 6.12, and Figure 6.13 furnish the $\mathrm{R}$ and G-curves at critical condition for SL-SF-L1-0.4, SL-SF-G2-0.4, and SL-SF-L1-0.3, respectively. The critical crack length $a_{c}$ and corresponding critical fracture energy $\left(R_{c}\right.$ or $G_{c}$ ) are also displayed in the figures. Substituting $a_{c}$ and $G_{c}$ into Eq. (6.9), the maximum allowable tensile strains for concrete rings at different ages can be obtained. Table 6.1 lists the results for all HPC mixtures tested.

Table 6.1 Maximum allowable tensile strains for HPC at different ages

\begin{tabular}{c|c|c|c|c|c|c}
\hline $\begin{array}{c}\text { Mixtures } \\
(1)\end{array}$ & $\begin{array}{c}\text { Ages } \\
(2)\end{array}$ & $\begin{array}{c}\text { Tensile } \\
\text { modulus of } \\
\text { elasticity } \\
(\mathrm{GPa}) \\
(3)\end{array}$ & $\begin{array}{c}a_{c} \\
(\mathrm{~mm}) \\
(4)\end{array}$ & $\begin{array}{c}G_{c} \text { or } R_{c} \\
(\mathrm{~N} / \mathrm{m}) \\
(5)\end{array}$ & $\begin{array}{c}\varepsilon_{\text {allowable }} \\
(\mu \varepsilon) \\
(6)\end{array}$ & $\begin{array}{c}\text { Max tensile } \\
\text { strain from } \\
\text { direst tensile test } \\
(\mu \varepsilon) \\
(7)\end{array}$ \\
\hline \multirow{4}{*}{ SL-SF-L1-04 } & 4 & 20.06 & 19.9 & 20.2 & 99 & 106 \\
\cline { 2 - 7 } & 7 & 21.58 & 30.8 & 46.1 & 102 & 105 \\
\cline { 2 - 7 } & 14 & 26.16 & 33.1 & 69.6 & 103 & $-*$ \\
\cline { 2 - 7 } & 28 & 28.63 & 35.6 & 96.6 & 110 & 114 \\
\hline \multirow{4}{*}{ SL-SF-G2-04 } & 4 & 20.23 & 22.3 & 16.2 & 85 & 100 \\
\cline { 2 - 7 } & 7 & 23.15 & 30.2 & 43.9 & 96 & 108 \\
\cline { 2 - 7 } & 14 & 25.62 & 26.9 & 60.0 & 114 & $-*$ \\
\cline { 2 - 7 } & 28 & 26.36 & 24.2 & 50.1 & 119 & 110 \\
\hline \multirow{5}{*}{ SL-SF-L1-03 } & 4 & 25.36 & 21.2 & 25.2 & 96 & 106 \\
\cline { 2 - 7 } & 7 & 28.12 & 25.0 & 50.8 & 112 & 104 \\
\cline { 2 - 7 } & 28 & 32.34 & 26.4 & 61.9 & 119 & $-*$ \\
\hline
\end{tabular}

Note : $1 \mathrm{~N} / \mathrm{m}=0.068 \mathrm{lbf} / \mathrm{ft}, 1 \mathrm{~mm}=0.0394 \mathrm{in}, 1 \mathrm{GPa}=145000 \mathrm{psi}$ )

* data are not available 
From Table 6.1, it is observed that the critical crack length $a_{c}$ ranges from $19.9 \mathrm{~mm}$ to $35.6 \mathrm{~mm}$ for three HPC mixtures tested. The variations in $a_{c}$ due to concrete ages are not definite, but there is trend of increasing $a_{c}$ with age with some inconsistencies. However, for very early age concrete (4-day), the $a_{c}$ is always lowest compared with other ages. The $6^{\text {th }}$ column in Table 6.1 is the max allowable strain obtained through the above numerical analysis procedure on the basis of fracture mechanics. The $7^{\text {th }}$ column is the tensile strain obtained from direct tensile tests as discussed in Chapter Four. Comparing those two columns, it is observed that in general at early age the tensile strains from fracture mechanics method are lower than those from the direct tensile test, and at later ages, the values are in general higher compared to the direct test results. The following reasons may explain those discrepancies:

1. The specimens used for direct tensile test are notched cylinders, while the specimens used for fracture mechanics analysis are notched beams and rings. It is believed that the size effect could be the main issue which results in differences of maximum strains obtained through these two approaches.

2. As has been discussed in Chapter Four, the so called "strength retrogression" which was caused by uneven drying and lead to stress concentration can reduce later age maximum strains in the direct tensile test. 

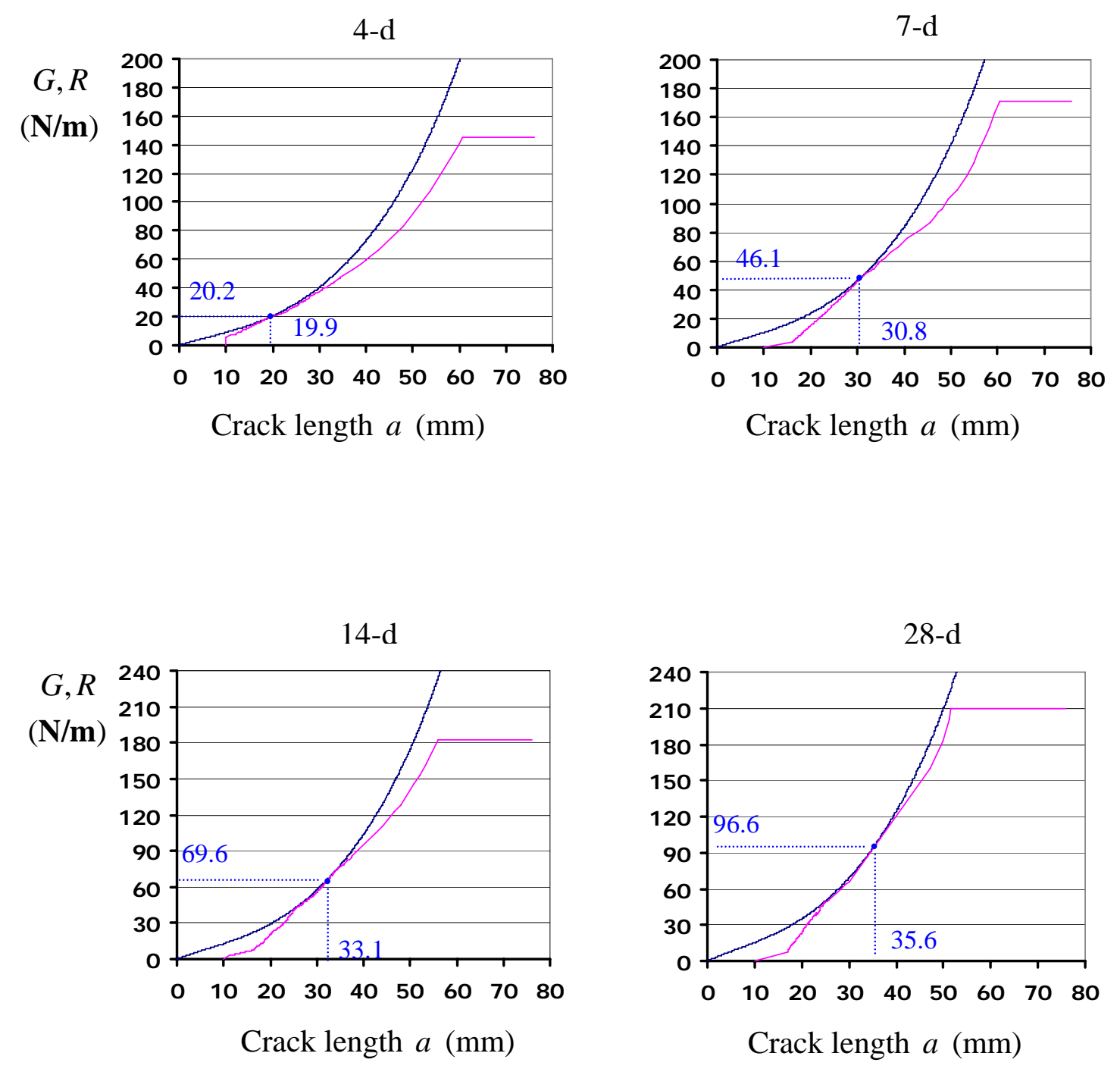

$$
\begin{aligned}
1 \mathrm{~N} / \mathrm{m} & =0.068 \mathrm{lbf} / \mathrm{ft} \\
1 \mathrm{~mm} & =0.0394 \mathrm{in}
\end{aligned}
$$

Figure 6.11 Critical crack lengths for SL-SF-L1-0.4 at different ages 

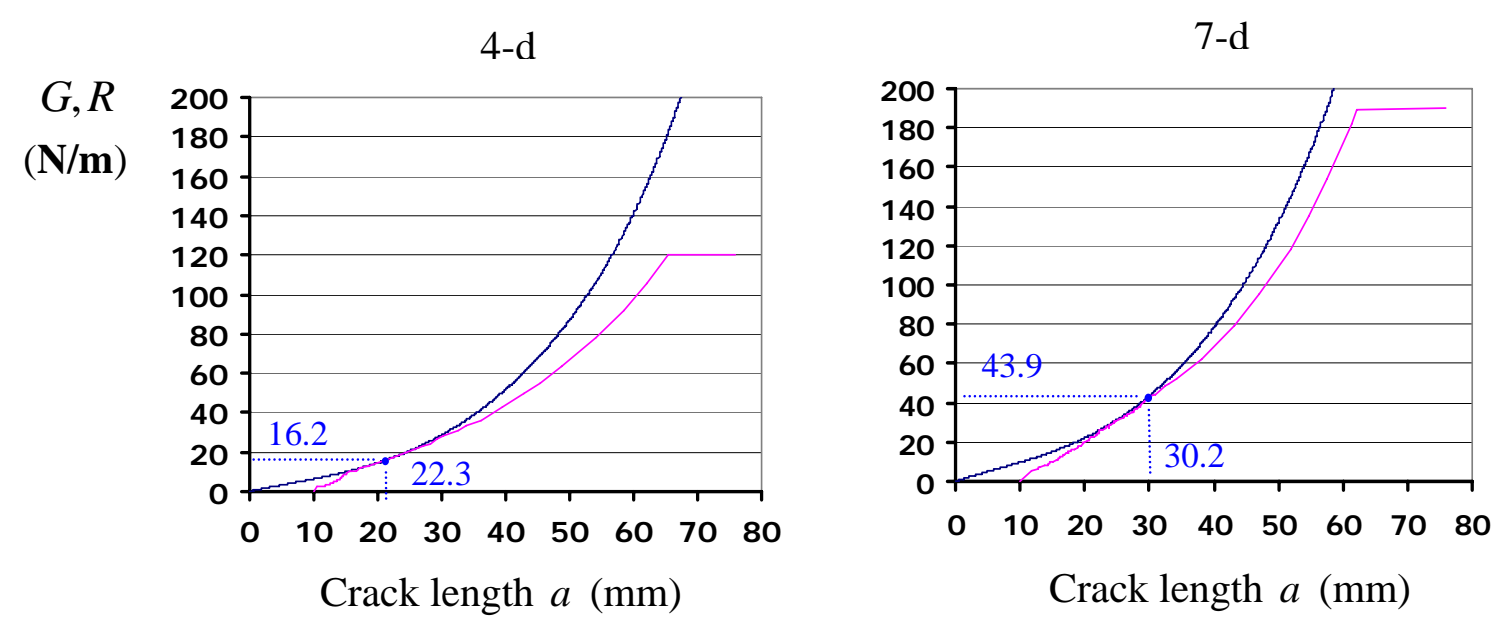

$14-d$
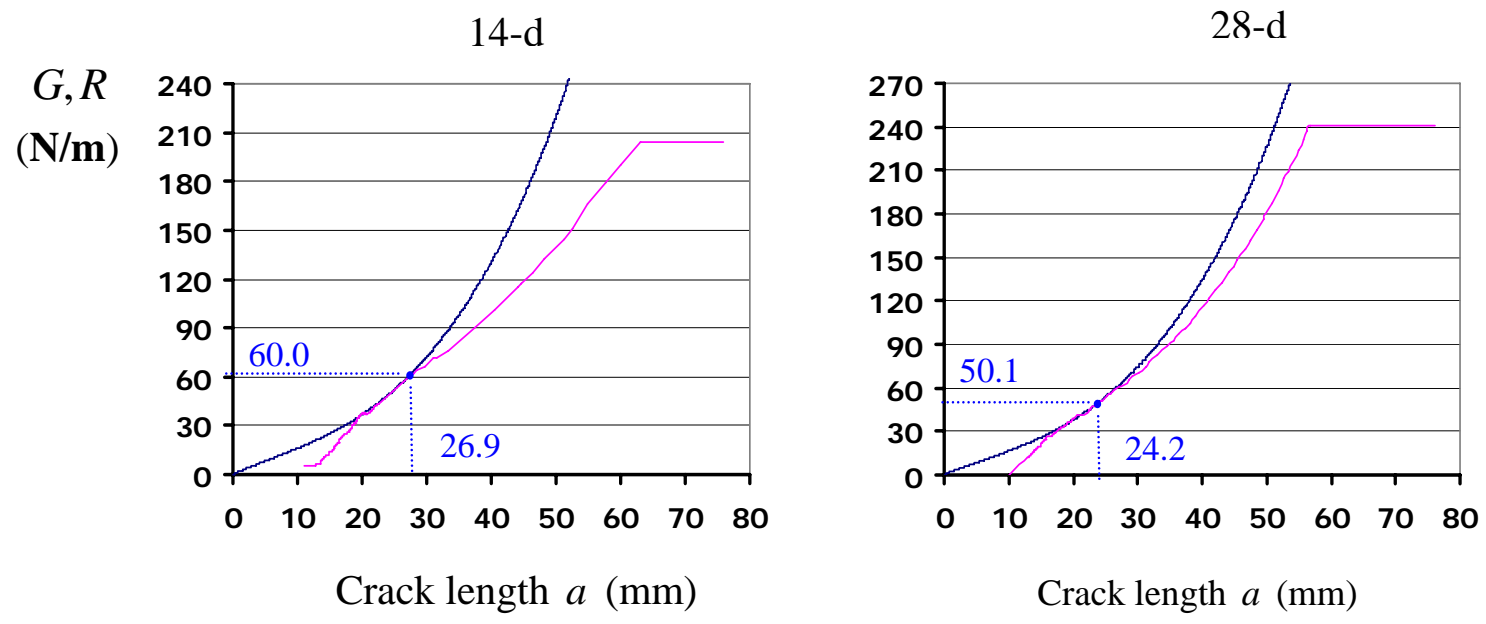

Crack length $a(\mathrm{~mm})$

$$
\begin{gathered}
1 \mathrm{~N} / \mathrm{m}=0.068 \mathrm{lbf} / \mathrm{ft} \\
1 \mathrm{~mm}=0.0394 \mathrm{in}
\end{gathered}
$$

Figure 6.12 Critical crack lengths for SL-SF-G2-0.4 at different ages 

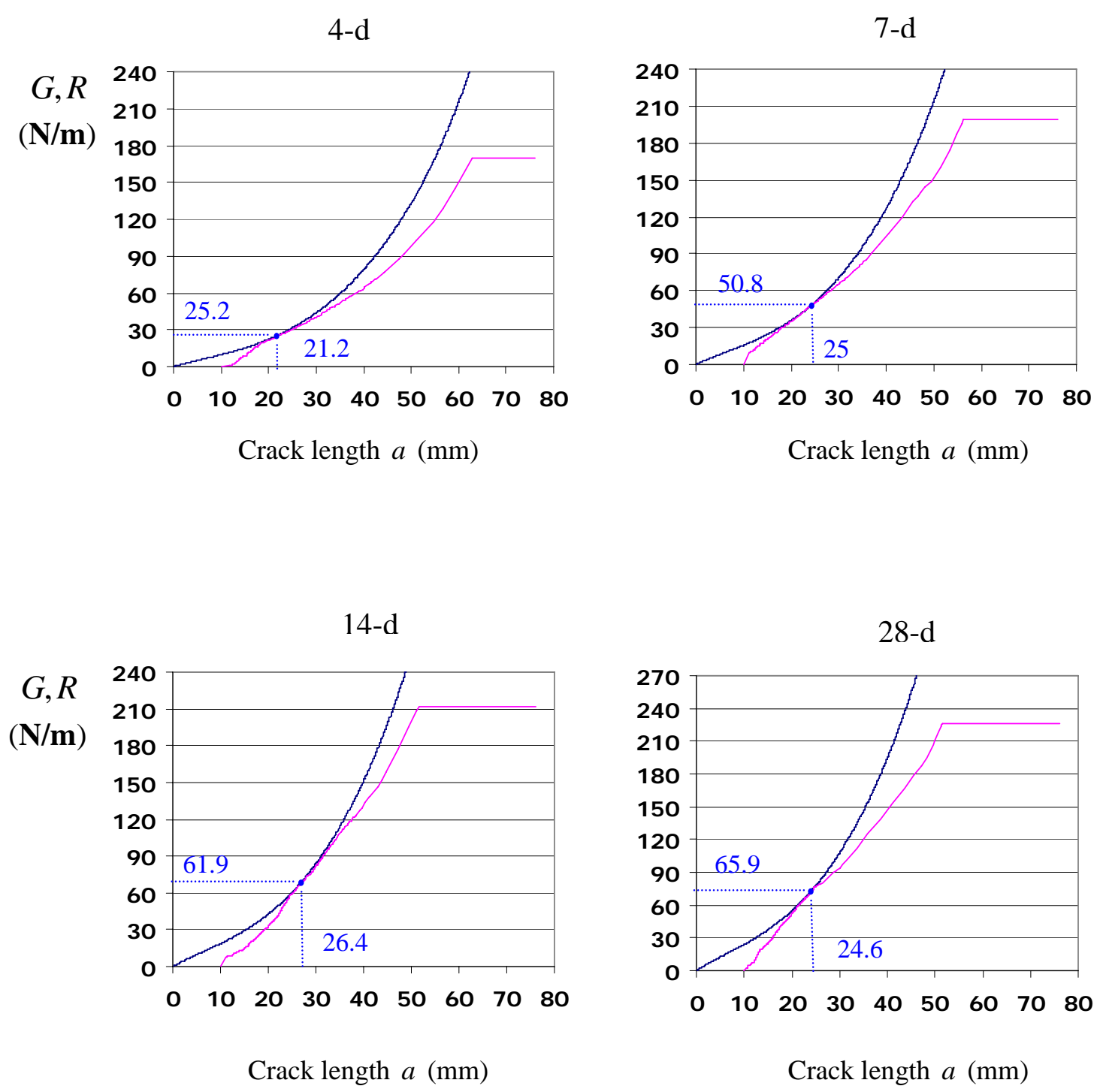

Crack length $a(\mathrm{~mm})$

Crack length $a$ (mm)

$$
\begin{gathered}
1 \mathrm{~N} / \mathrm{m}=0.068 \mathrm{lbf} / \mathrm{ft} \\
1 \mathrm{~mm}=0.0394 \mathrm{in}
\end{gathered}
$$

Figure 6.13 Critical crack lengths for SL-SF-L1-0.3 at different ages 


\subsection{Prediction of Cracking Onset}

\subsubsection{Estimation of creep}

The cracking of the ring specimen results from the difference of free shrinkage and creep. The free shrinkage was measured with prism specimens, which had same the exposure of surface to volume ratio as those of the ring specimens. The details of free shrinkage test have been discussed in Chapter Four. It is necessary to estimate the magnitude of creep which simultaneously develops with the shrinkage of the ring specimens. In this study, the RILEM B3 model developed by Bazant and Prasannan (1989) was adopted as the method to estimate the tensile creep for ring specimen. For a constant stress applied at age $t^{\prime}$, the total strain can be calculated as:

$$
\varepsilon(t)=J\left(t, t^{\prime}\right) \sigma+\varepsilon_{s h}(t)
$$

where $t$ is age at which creep is evaluated; $\sigma$ is the uniaxial tensile stress; $\varepsilon_{\text {sh }}$ is the free shrinkage, and $J\left(t, t^{\prime}\right)$ is creep compliance which can be expressed as:

$$
J\left(t, t^{\prime}\right)=q_{1}+C_{0}\left(t, t^{\prime}\right)+C_{d}\left(t, t^{\prime}, t_{0}\right)
$$

where $q_{1}$ is the instantaneous strain due to unit stress; $C_{0}\left(t, t^{\prime}\right)$ is the compliance for basic creep (creep at constant moisture content), and $C_{d}\left(t, t^{\prime}\right)$ is additional creep compliance due to simultaneous drying.

The instantaneous strain $q_{1}$ may be expressed as $1 / E_{0}$, where $E_{0}$ is the asymptotic modulus. In the B3 model, $E_{0}$ is assumed to be equal to $E_{28}$, where $E_{28}$ is the modulus of elasticity at 28-day. 
The basic creep compliance can be expressed as

$$
C_{0}\left(t, t^{\prime}\right)=q_{2} Q\left(t, t^{\prime}\right)+q_{3} \ln \left[1+\left(t-t^{\prime}\right)^{0.1}+q_{4} \ln \left(\frac{t}{t^{\prime}}\right)\right.
$$

where $q_{2}$ is the ageing viscoelastic compliance; $q_{3}$ is non-ageing viscoelastic compliance; $q_{4}$ is flow compliance, and $Q\left(t, t^{\prime}\right)$ is a binomial integral which can be calculated approximately or by numerical integration.

$q_{2}, q_{3}, q_{4}$ can be calculated as follows

$$
\left.\begin{array}{l}
q_{2}=451.1 c^{0.5}\left(f_{c}^{\prime}\right)^{-0.9} \\
q_{3}=0.29(w / c)^{4} q_{2} \\
q_{4}=0.14(a / c)^{-0.7}
\end{array}\right\}
$$

where $\mathrm{c}$ is the cement content of concrete; $f_{c}^{\prime}$ is the 28 day standard cylinder compression strength; w/c is water to cement ratio, and a/c is aggregate to cement ratio. The B3 prediction model is restricted to portland cement concretes with material parameter ranges as follows: $17.3 \leq f_{c}^{\prime} \leq 69, \quad f_{c}^{\prime} \quad$ in $\mathrm{MPa} ; \quad 0.3 \leq w / c \leq 0.85$; $160 \leq c \leq 720, \quad c$ in $\mathrm{kg} / \mathrm{m}^{3}$, and $2.5 \leq a / c \leq 13.5$.

The additional drying creep compliance $C_{d}\left(t, t^{\prime}, t_{0}\right)$ can be calculated as:

$$
C_{d}\left(t, t^{\prime}, t_{0}\right)=q_{5}\left\{\exp [-8 H(t)]-\exp \left[-8 H\left(t^{\prime}\right)\right]\right\}^{1 / 2}
$$

where

$$
\begin{aligned}
& \mathrm{H}=1-(1-\mathrm{h}) S(\mathrm{t}) \\
& q_{5}=7.57 \times 10^{5} f_{c}^{\prime-1} \varepsilon_{\text {shoo }}^{-0.6}
\end{aligned}
$$

where h is relative humidity ( $h=50 \%$ in this study); $S(t)$ is time function for shrinkage (as expressed in Eq. 4.14), and $\varepsilon_{\text {shoo }}$ is ultimate shrinkage strain, which can be found in Table 4.2. 
The calculation procedure described above can be programmed easily to tabulate creep compliance $J\left(t, t^{\prime}\right)$ for concrete loaded at different time $t^{\prime}$. The Appendix E gives the $J\left(t, t^{\prime}\right)$ values for different HPC mixtures till 24 days. The cement content $c$ in Eq. (6.20) is assumed as cementitious material in this study.

As mentioned before, the circumferential stress $\sigma_{\theta \theta}$ resulting from shrinkage inside the ring specimen changes with time, and therefore, the integral form should be used for ring specimen, as

$$
\varepsilon_{c}(t)=\int_{0}^{t} J\left(t, t^{\prime}\right) d \sigma_{\theta \theta}\left(t^{\prime}\right)
$$

where $\varepsilon_{c}(t)$ is the circumferential strain, and is the sum of elastic strain and creep strain.

Equation (6.24) can be approximately calculated by the following numerical approach:

$$
\varepsilon_{c}(t)=\sum_{t_{i}=1}^{t} J\left(t, t_{i}^{\prime}\right) \Delta \sigma_{\theta \theta}\left(t_{i}^{\prime}\right)
$$

In Eq. (6.25), the total stress can be divided into several small increments of $\Delta \sigma_{\theta \theta}$, at the time interval which is the same as for free shrinkage measurement. The total strain $\varepsilon_{c}$ at time $t$ is the sum corresponding to every stress increment according to the principle of superposition.

Figures 6.14 (a), (b), and (c) show free shrinkage, creep, and free shrinkage minus creep development versus time for SL-SF-L1-0.4, SL-SF-G2-0.4, and SL-SF-L1-0.3, respectively. 


\subsubsection{Prediction of cracking onset}

The maximum allowable strains obtained previously, as well as the induced strains (by considering the creep with time) were plotted in the same figure, and the intersection of those two figures was regarded as the cracking onset time, as shown in Figure 6.15. Figure 6.15 (a), (b), and (c) represent SL-SF-L1-0.4, SL-SF-G2-0.4, and SL-SF-L1-0.3, respectively. Table 6.2 lists the predicted cracking onset time as well as the measured cracking onset time.

Table 6.2 Comparison of predicted values with measured values

\begin{tabular}{c|c|c|c}
\hline \multirow{2}{*}{ Mixture } & \begin{tabular}{c} 
Predicted \\
cracking onset \\
time \\
\cline { 3 - 4 } (days)
\end{tabular} & \multicolumn{2}{|c}{$\begin{array}{c}\text { Measured cracking onset time } \\
\text { (days) }\end{array}$} \\
\cline { 3 - 4 } & 16 & 12 & 14 \\
\hline SS-L1-0.4 & 24 & 16 & 21 \\
\hline SS-G2-0.4 & 9 & 8 & 8 \\
\hline SS-L1-0.3 & 9 & Specimen 1 & \\
\hline
\end{tabular}

Table 6.2 shows that the predicted cracking onset time for HPC mixtures with w/cm of 0.4 (SL-SF-L1-0.4 and SL-SF-G2-0.4) are somewhat larger than the measured cracking onset time, and for HPC with w/cm $=0.3$ (SL-SF-L1-0.3), the predicted onset day agrees well with the measured value.

From the numerical analysis as well as the results from laboratory tests, it may be concluded that the AASHTO ring specimen test is an effective way to evaluate the cracking properties of different HPC mixtures (for both limestone and gravel aggregates as well as HPC with w/cm as low as 0.3 ). The restrained cracking can be evaluated by ring test with the reasonable accuracy. Since all the predicted cracking days are higher than the measured values, the estimation by ring test is slightly conservative. The restraint provided by the steel ring is enough to cause the cracking of the concrete ring. 


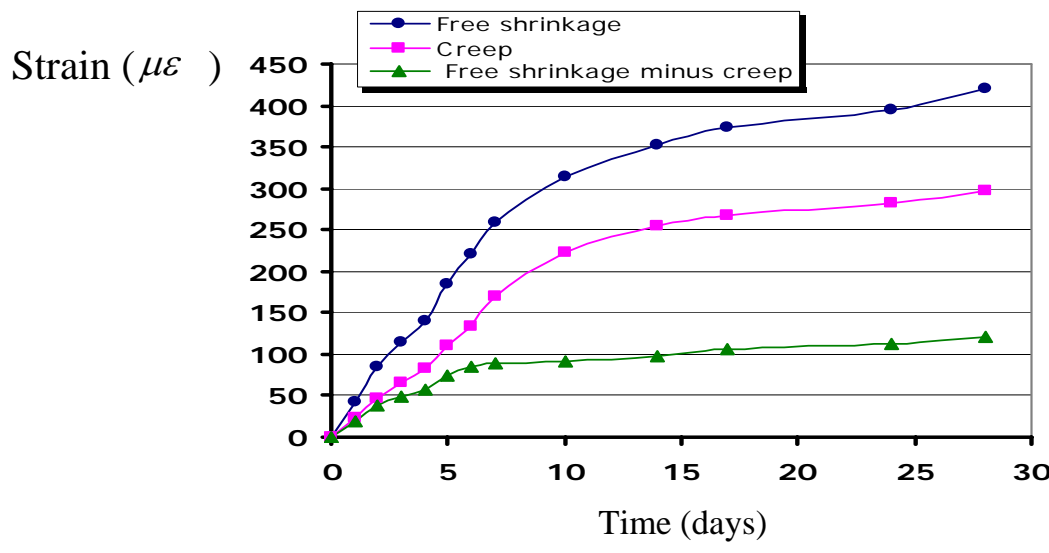

(a) SL-SF-L1-0.4

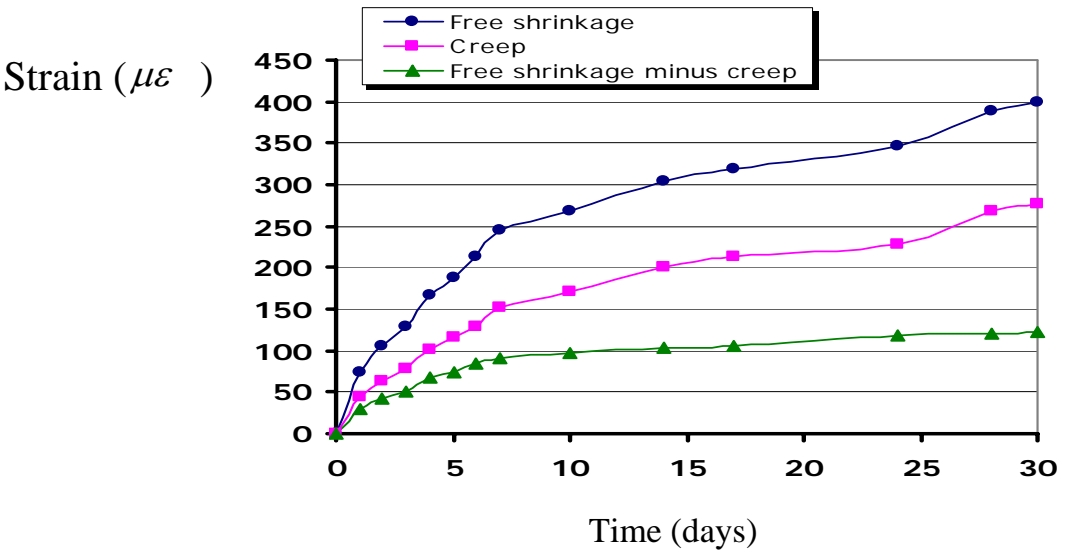

(b) SL-SF-G2-0.4

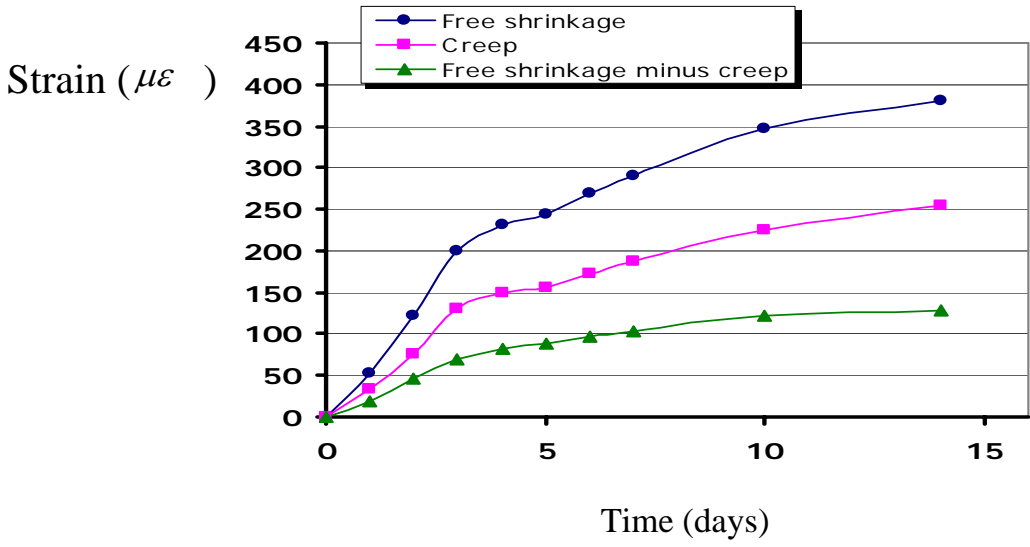

(c) SL-SF-L1-0.3

Figure 6.14 Free shrinkage, creep and free shrinkage minus creep development versus time 


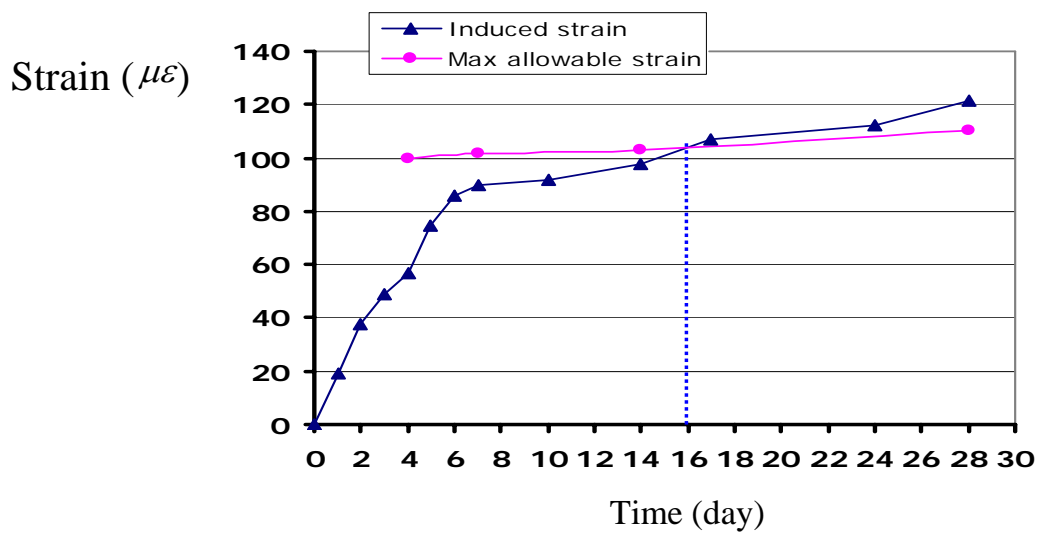

(a) SL-SF-L1-0.4

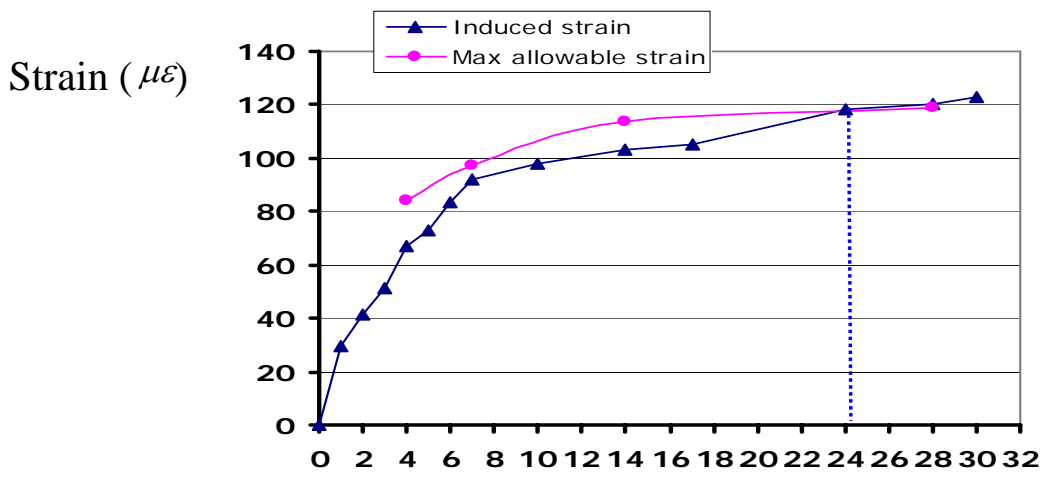

Time (day)

(b) SL-SF-G2-0.4

Strain $(\mu \varepsilon)$

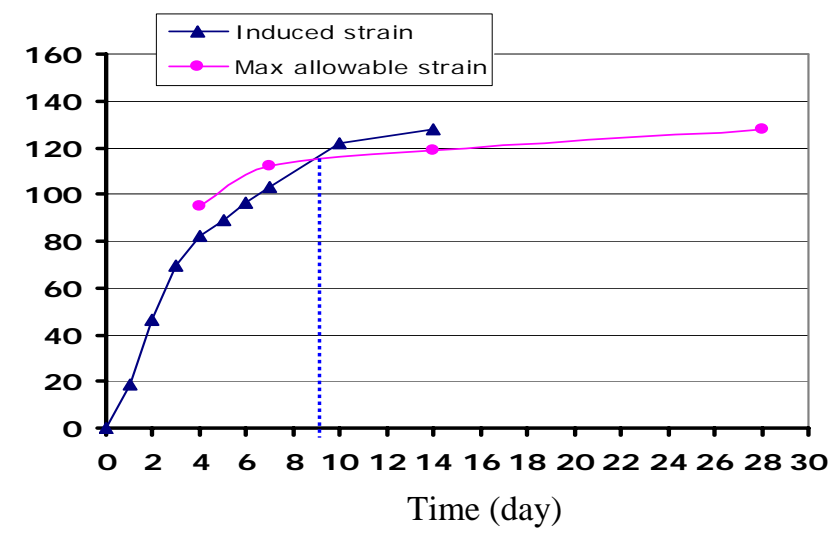

(c) SL-SF-L1-0.3

Figure 6.15 Theoretical predictions of cracking onset days for ring specimens 


\subsection{Concluding Remarks}

1. A numerical procedure based on fracture mechanics was developed for predicting the transverse cracking of AASHTO ring specimens due to drying shrinkage. The predicted crack onset day is reasonably in agreement with the experimental results.

2. This numerical model provided a theoretical basis for the AASHTO ring restrained shrinkage test. Based on the experimental results as well as the numerical analysis, it can be concluded that the AASHTO ring test is an effective approach for cracking evaluation of different HPC mixtures.

3. It was found that the critical crack length $a_{c}$ ranges from $19.9 \mathrm{~mm}$ to $35.6 \mathrm{~mm}$ for three HPC mixtures tested. The variations in $a_{c}$ due to concrete ages are not definite, but there is trend of increasing $a_{c}$ with age with some inconsistencies. However, for very early age concrete (4-day), the $a_{c}$ is always lowest compared to other ages.

4. Due to the size effect from different approaches, at early age, the maximum tensile strains obtained from fracture mechanics approach were lower than those from direct tensile test, while at later ages, the values were higher compared to the direct test results

5. Bazant's B3 creep model provided reasonable estimations of creep values for different HPC mixtures in this study. However, in order to obtain more precise results, more research work is needed for the tensile creep property of HPC with different SCM. Also, more types of HPC should be studied for the comparison of their predicted values with measured values. 


\section{CHAPTER SEVEN}

\section{FRACTURE MECHANICS TEST}

\subsection{Introduction}

In Chapter Six, a numerical analysis procedure based on fracture mechanics was developed. This procedure has shown reasonable predictions on the cracking of ring specimens. Unlike the traditional strength based theory, the fracture mechanics approach considers the failure to be propagating rather than occurring simultaneously throughout the structure. There are valid reasons to believe that fracture mechanics has advantages over the conventional failure criteria, which are based on elasticity or plasticity theory. ACI 446 1R-91 (1991) listed five reasons in favor of the inclusion of the theory of fracture mechanics into the future structural design codes. A brief review of those reasons is as follows:

\section{(a) Energy required for crack formation}

Concrete contains numerous flaws and microcracks. The crack initiation may depend on the level of stress, however, the actual crack formation and growth requires certain amount of energy, and therefore, the energy criteria should be employed.

\section{(b) Objectivity of analysis}

The finite element method (FEM) has been a well-established and effective technique for the analysis of concrete structures and cracking. One concern with the FEM is that the analytical results can significantly change if the mesh is refined (Rashid, 1962). This is because the energy, which is dissipated due to cracking, decreases with the refinement of the finite element, even after the inclusion of the tension softening response. The only 
way to avoid it is to introduce the concept of fracture energy, i.e. a constant energy of dissipation independent of the element size.

\section{(c) Lack of yield plateau}

Based on load-displacement response, there are two basic types of failure models: plastic and brittle. The plastic failure is characterized by a plateau in the load-deflection diagram, as shown in Figure 7.1 (a). The failure of the structure happens simultaneously when sufficient plastic hinges are formed. For brittle or quasi-brittle material, as shown in Figure 7.1 (b), the structures usually show softening responses (due to flaws cracking, and other damages). The failure consists of propagation of the failure zone and is nonsimultaneous. The plastic analysis may overestimate the failure load if it is applied for brittle or quasi-brittle materials.

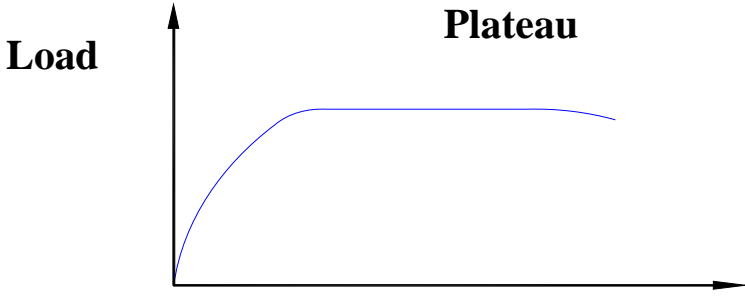

Deflection

(a)

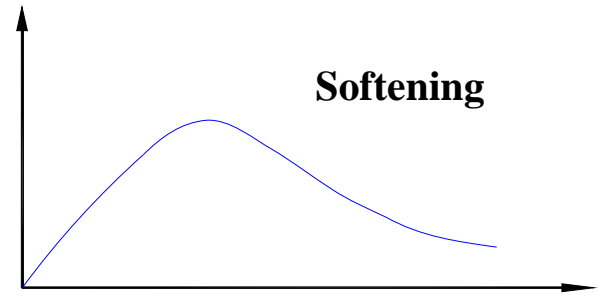

Deflection

(b)

Figure 7.1 Load and deflection responses of ductile and brittle structures

\section{(d) Energy absorption capability and ductility}

When the dynamic and seismic analysis of the structures is considered, the energy absorption capacity of the structure is a very important parameter. For this reason, some form of fracture mechanics is necessary. 


\section{(e) Size effect}

The most compelling reason in favor of fracture mechanics is the so called size effect. The classical theory uses strength limit or failure criterion in terms of stresses, in which the normal stress $\sigma_{N}$ is constant for any size of the structures, as shown in Figure 7.2 by a horizontal line. But it is known that the larger the size of the specimen, the smaller is the value of $\sigma_{N}$. If the failure is governed by fracture mechanics, $\sigma_{N}$ will decrease with the increase of structure size as illustrated in the Figure 7.2.

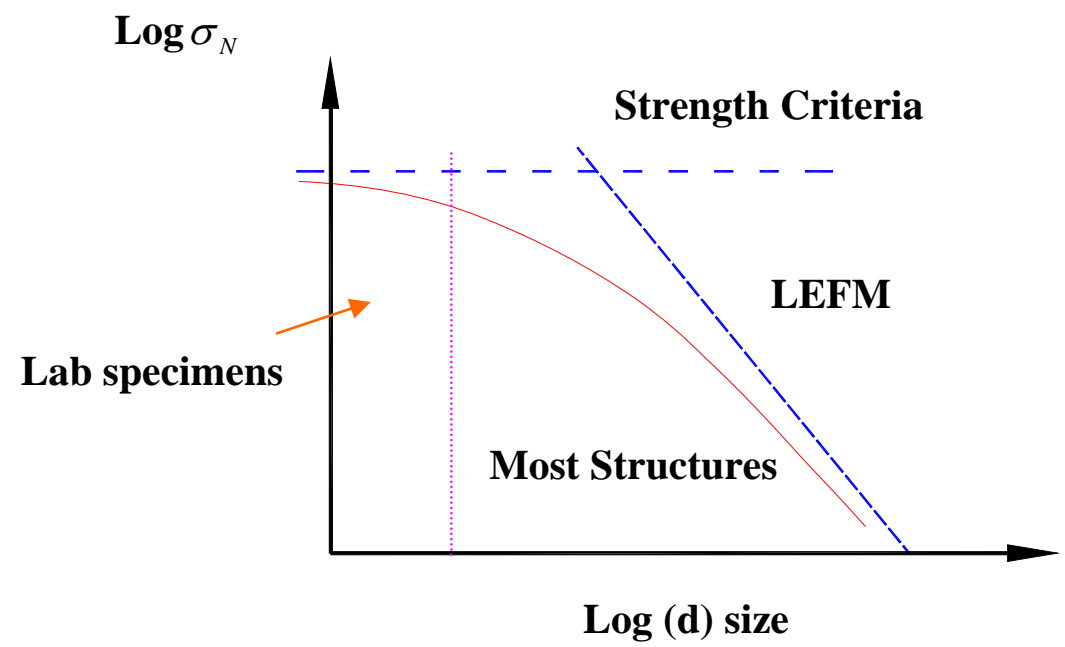

Figure 7.2 Size effect of geometrically similar structures with different size (Adopted from: ACI 446, 1991)

Since 1980s, the investigations about the fracture mechanics of quasi-brittle materials like concrete, rocks, and ceramics have grown enormously. Several theoretical models have been established. Those models can be classified into two different categories: effective elastic crack models (modified linear elastic fracture mechanics), and cohesive crack models. The first category includes the two-parameter model (TPM) by Jenq and Shah (1985), size effect model (SEM) by Bazant (1990), and effective crack model (ECM) by Karihalloo and Nallathambi (1989). The fictitious crack model (FCM) by 
Hillerborg (1976), and crack band model (CBM) by Bazant (1983) belong to the second category. A brief description of those models can be found in Chapter Two. Each model has sound theoretical basis and shortcomings. Until now, no model has been accepted as a general guideline that can be applied in all cases. More studies are needed for a better understanding of the fracture mechanics of concrete.

Though several studies have been conducted on HPC, most of them were focused on conventional properties like strength, shrinkage and durability issues. It is known that HPC, due to the use of the chemical admixture and SCM, has an improved pore structure and denser paste-aggregate transition zone, which in turn affect the fracture characteristics. However, only limited research information can be found on the fracture mechanics properties of HPC.

Niwa and Tangermsirikul (1997) conducted fracture mechanics tests on selfcompacting HPC produced with fly ash. The fracture energy of different HPC mixtures were measured and compared with conventional concrete. Kjellsen et al. (2000) studied the fracture mechanics properties of HPC containing 10\% silica fume and different w/cm. The tests lasted for 2 years and fracture energies at 28-day and 2-year for different mixtures were obtained. Bharatkumar et al. (2005) studied the influence of fly ash and slag on the fracture characteristics of HPC. In their study, the Hillerborg's FCM and Bazant's SEM were employed to determine the fracture energies, and the test results based on those two methods were compared. Since only limited information was found on the fracture mechanics properties of HPC, a further study on the fracture properties of the HPC is needed.

In Chapter Six, three HPC mixtures (SL-SF-L1-0.4, SL-SF-G2-0.4, and SL-SF-L10.3) were selected for the fracture mechanics tests to obtain the fracture energy and P- 
$C M O D$ responses. However, the size of the specimens used for those tests was designed to construct R-curves corresponding to the size of the ring specimens, and did not follow any standard test requirements. In this section, those three HPC mixtures as well as other three HPC mixtures were selected for fracture mechanics tests, which are based on the Hillerborg's FCM method as suggested by RILEM committee FMC 50.

\subsection{Testing Procedure}

Hillerborg's (1985) work force method (WFM) with three-point bending, and singleedge notch (TPB-SEN) beam was adopted in this study. A brief introduction of Hillerborg's test method was given in Chapter Two.

\subsubsection{Materials and beam size}

A total of six different HPC mixtures were selected from the original 24 HPC mixtures. Those are as follows:

1. SL-SF-L1-0.4 (Slag + silica fume with limestone 1 and $\mathrm{w} / \mathrm{cm}=0.4$ );

2. MK-L1-0.4 (Metakaolin with limestone 1 and $\mathrm{w} / \mathrm{cm}=0.4$ );

3. SL-SF-G2-0.4 (Slag + silica fume with gravel 2 and w/cm = 0.4);

4. MK-G2-0.4 (Metakaolin with gravel 2 and w/cm = 0.4);

5. SL-SF-L1-0.3 (Slag + silica fume with limestone 1 and w/cm = 0.3);

6. MK-L1-0.3 (Metakaolin with limestone 1 and $\mathrm{w} / \mathrm{cm}=0.3$ ).

The coarse aggregates used in this study were graded limestone and gravel, which had a maximum size $d_{\max }=25 \mathrm{~mm}$ ( 1 in). According to RILEM committee FMC 50 recommendation (1985), for coarse aggregates with maximum size between 16.1 $32 \mathrm{~mm}$ (0.6 in $1.25 \mathrm{in}$ ), the depth of beam should be $200 \mathrm{~mm} \pm 5 \mathrm{~mm}$ (8 in $\pm 0.2 \mathrm{in})$. Based on this recommendation, the beam was designed as shown in Figure 7.3. The length of the 
beam was $1200 \mathrm{~mm}$ (47 in); the span was $813 \mathrm{~mm}$ (32 in), and the span-to-depth ratio was equal to 4. The notch depth was equal to half of the beam depth, which was $100 \mathrm{~mm}$ (4 in) for all the beams tested.
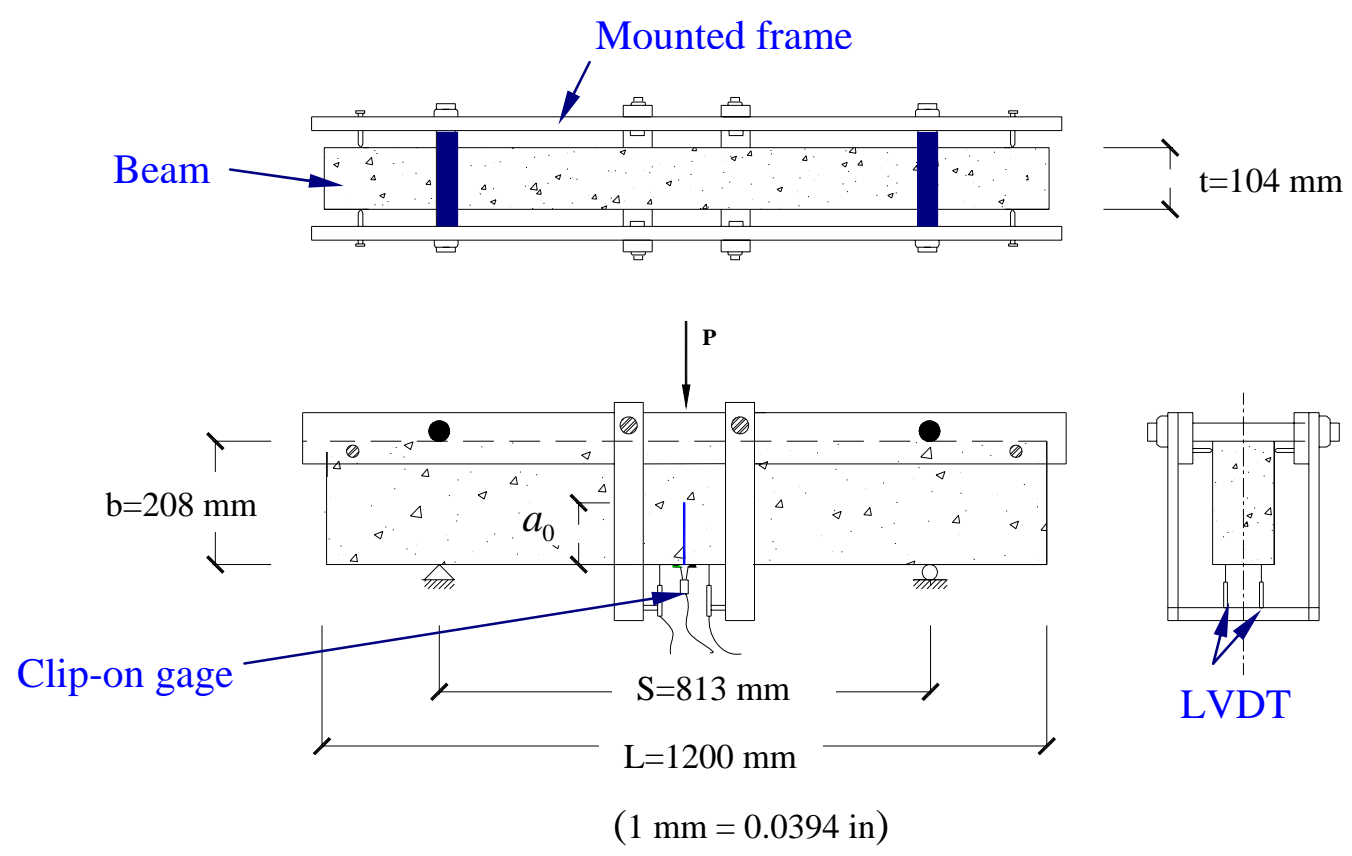

Figure 7.3 Sketch of the beam and displacement transducer frame

\subsubsection{Testing setup}

In order to achieve a stable test, the testing system is required to be stiff enough or a closed-loop servo control system. In this study, a rigid steel frame as well as an MTS loading actuator was used as the testing system. The overview of the frame and testing system is illustrated in Figure 7.4 (b).

Based on the results of theoretical stability analysis, Hillerborg (1985) proposed the stiffness requirements of testing frames for beam specimens with different dimensions. For the beam size employed in this test, the minimum stiffness requirement should be at least $25 \mathrm{kN} / \mathrm{mm}$ (142 kips/in). In order to estimate the stiffness of the frame built for this test, a numerical analysis of the frame was conducted using a frame analysis program 
(RISA Technologies, 2001). The frame is simplified as a 3-D model as illustrated in

Figure 7.4 (a). A unit load was applied at the point where the actuator was to be mounted, and the inverse of the corresponding displacement was taken as the stiffness of the frame. According to the results of the analysis, the stiffness of frame was found to be round 30 $\mathrm{kN} / \mathrm{mm}$ (171 kips/in), which was larger than the minimum requirement $25 \mathrm{kN} / \mathrm{mm}$ (142 kips/in) as suggested by Hillerborg. Thus, the frame was believed to be stiff enough to perform a stable test.

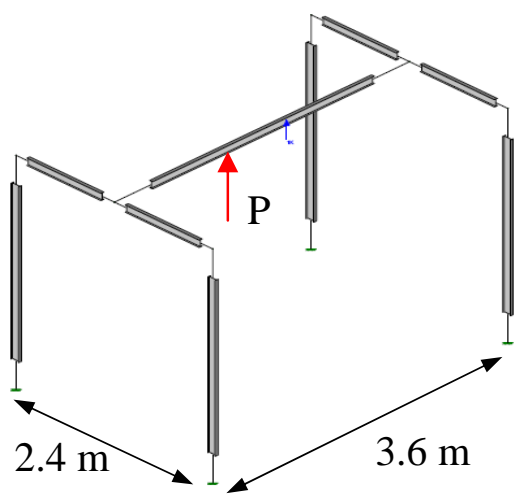

(a)

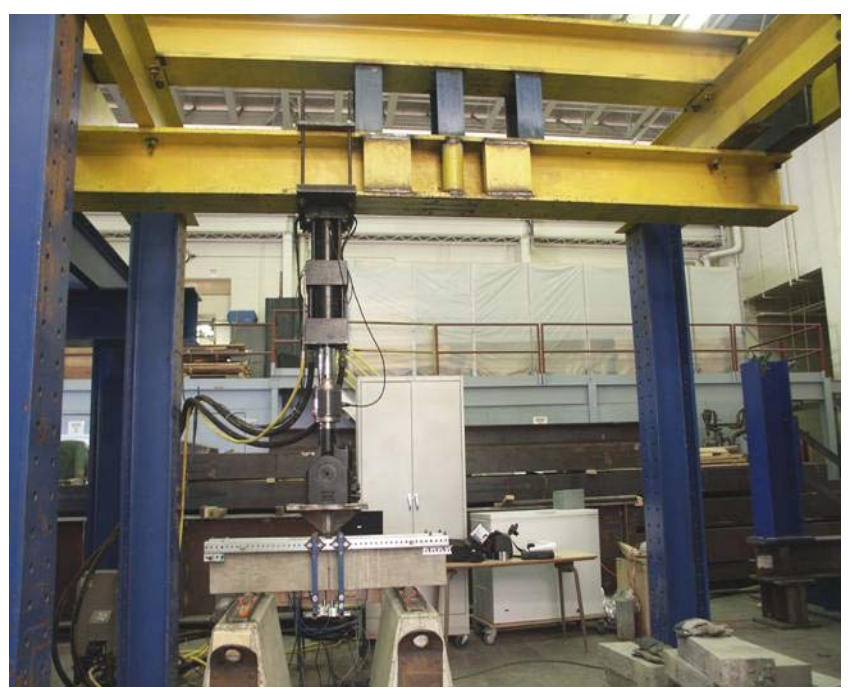

(b)

Figure 7.4 The testing frame

The MTS hydraulic actuator was calibrated for a maximum load capacity of $24.5 \mathrm{kN}$ (5.5 kips) and a maximum displacement capacity of $152 \mathrm{~mm}$ (6 in). The accuracies of the load and displacement were $10 \mathrm{~N}(2.2 \mathrm{lb})$ and $0.2 \mathrm{~mm}(0.008 \mathrm{in})$ respectively. The actuator was controlled by an MTS 407 controller with a displacement-control mode.

The test beams were simply supported using steel rollers at both sides. The load was applied at the mid-span of the beam through a cylindrical steel bar welded to the spherical grip of the actuator. Two displacement transducers (LVDTs) were used to 
record the load point displacement. The transducers were mounted at the bottom of the beam (one for each side of the beam) through a specially designed transducer frame. As shown in Figure 7.3, the displacement transducer frame was placed over the top of the beam with two cylindrical steel bars resting at the locations corresponding to the end beam supports. The purpose was to minimize the influence of the rotation of the beam during the loading process. A clip-on gage was mounted on two knife edges over the notch at the midway along the beam width. The CMOD was measured by the clip-on gage. The accuracies of the displacement transducers and clip-on gage were $0.01 \mathrm{~mm}$ (0.0004 in) and $0.005 \mathrm{~mm}$ (0.0002 in), respectively. The readings of the load, stroke of actuator, two displacement transducers, and clip-on gage were recorded automatically by a data acquisition system. Figures 7.5 (a) (b) and (c) show an overview of the testing system with data acquisition system, a front view of the beam under the loading, and a close view of displacement transducers and clip-on gage setup, respectively.

\subsubsection{Testing procedure}

The concrete beam specimens were prepared and cast following the same procedures used in previous tests, such as direct tensile test and ring test. All of the beams were cured within the mold by covering with wet burlap for 24 hours. After demolding, the beams were moved to the environmental chamber at a temperature of $23{ }^{\circ} \mathrm{C}\left(73^{\circ} \mathrm{F}\right)$, and relative humidity of $50 \%$ till the day of testing. The tests were conducted at the ages of 4 , 7, 14, and 28-day. For each age, three replicate specimens were tested.

A few hours before the test, the beam specimens were taken out from the environmental chamber. The notches were cut by a diamond saw with high accuracy. The notched beams were then transported with care to the structure lab where all the fracture tests were conducted. Figure 7.6 shows typical notched beams ready for testing. 


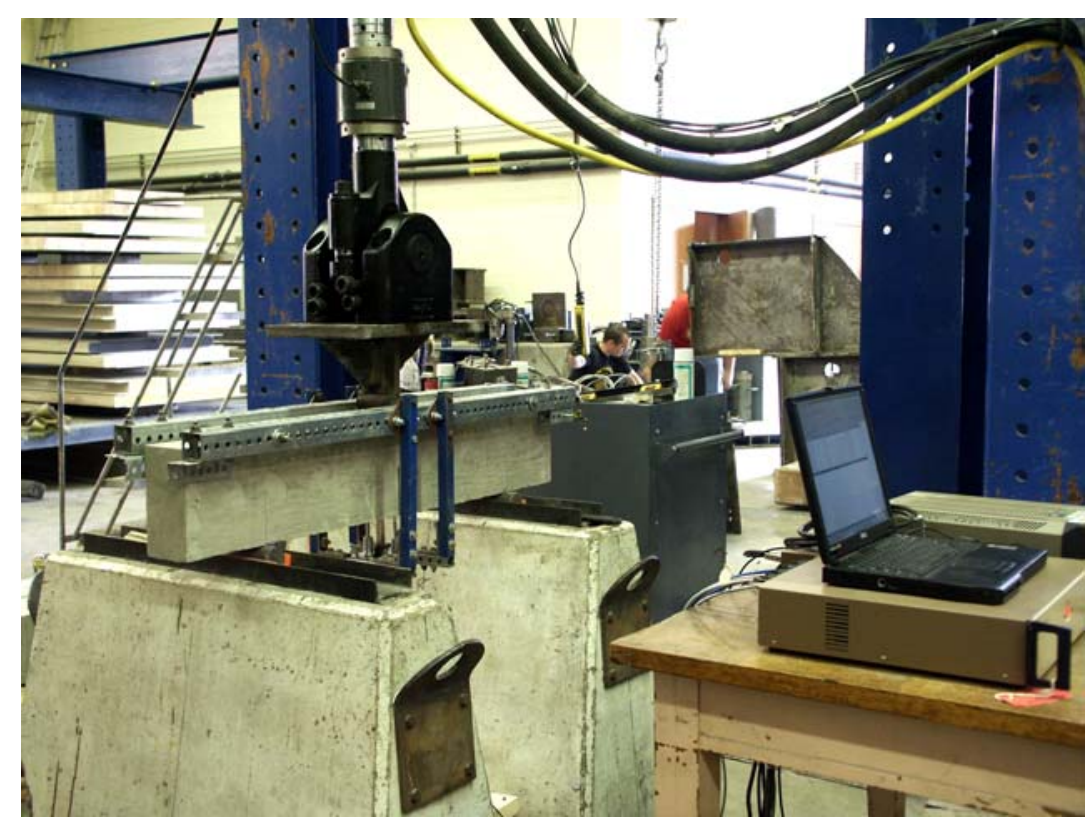

(a)

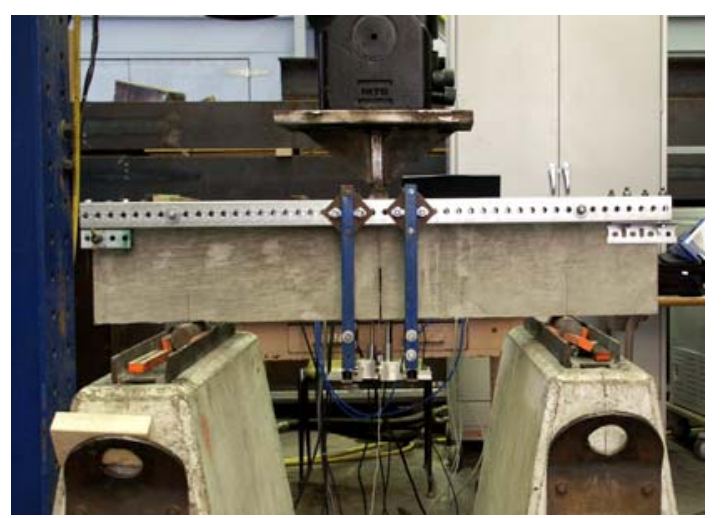

(b)

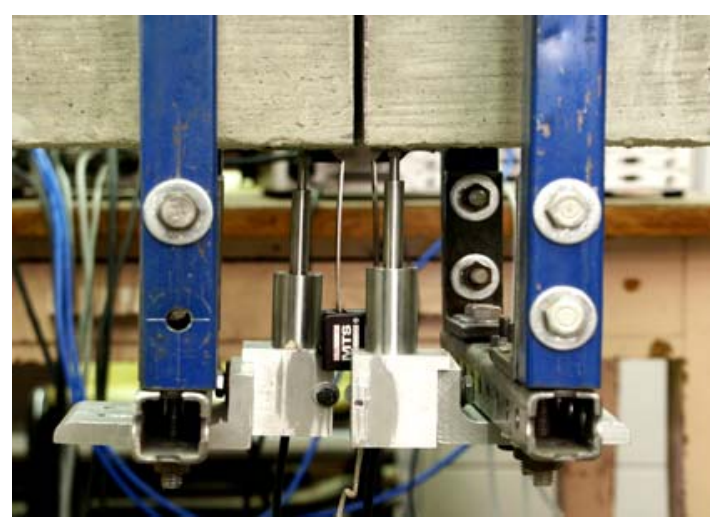

(c)

Figure 7.5 The test setup 


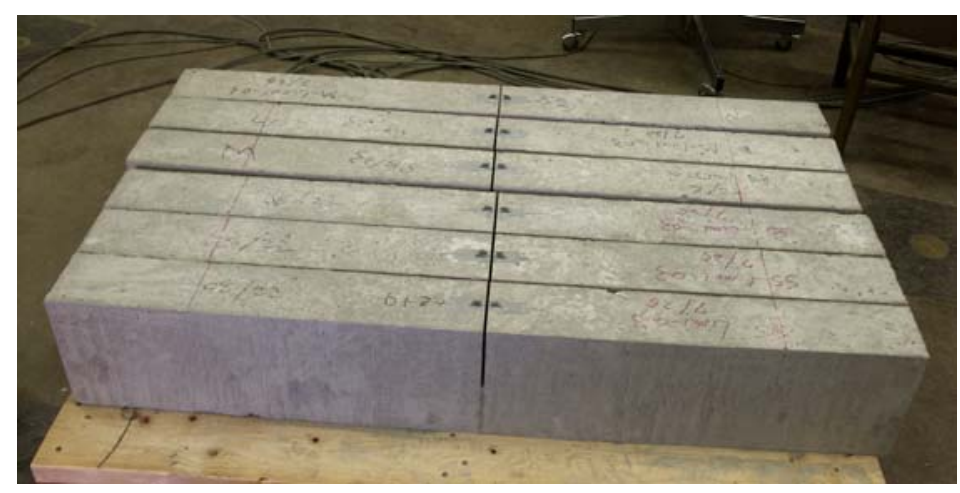

Figure 7.6 The notched beams

All the tests were conducted under stroke displacement control mode. Four measurements were recorded: (1) applied load, (2) stroke, (3) mid-span displacement (MSD), and (4) CMOD. The Load-MSD diagrams were displayed during testing on the computer monitor. The peak load was reached within 3 minutes, and the whole test was completed within 8 minutes. The test was terminated at the time when either the midspan displacement reached around $1.4 \mathrm{~mm}$, or the load reduced to a value less than $20 \mathrm{~N}$. The above criteria were selected to terminate the test, because under those circumstances the beam was believed to be completely fractured.

\subsubsection{Validation of the testing system}

Before conducting the formal test, several mock tests were observed using the testing setup designed for this study in order to validate the whole system. The following observations were made:

1) The frame was rigid enough to perform a stable test, i.e. to obtain a stable postpeak load-displacement response.

2) The data acquisition system was able to record the readings of displacement transducers and clip-on gauge with reasonable accuracy. 


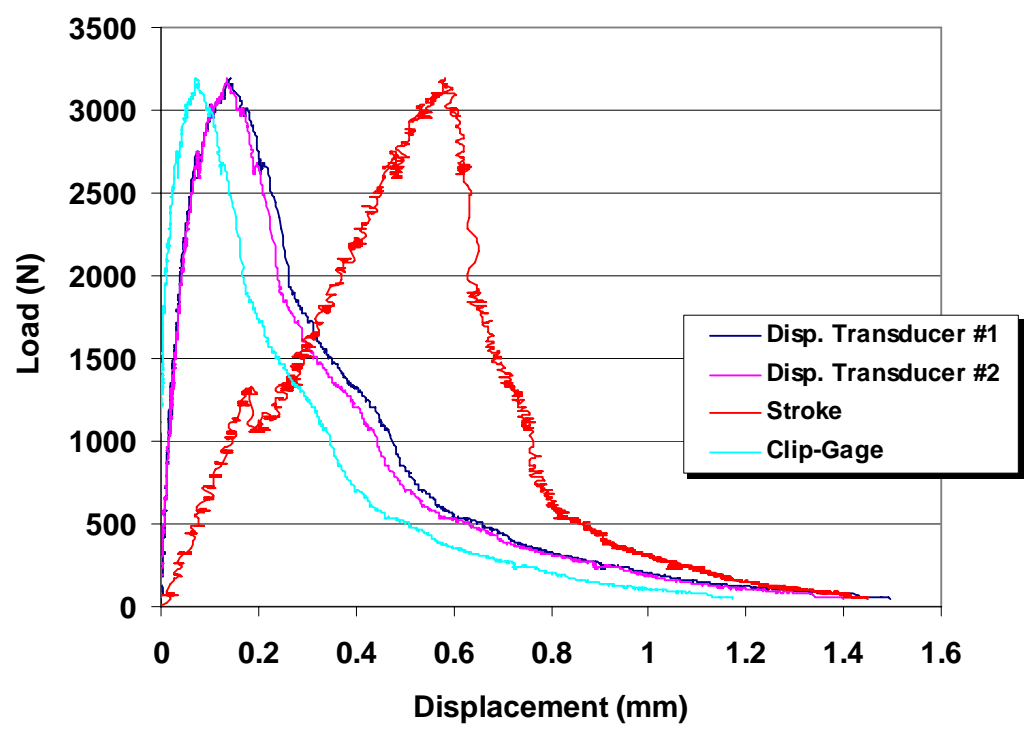

Figure 7.7 Typical load-displacement curves

$$
\text { (1 } \mathrm{N}=0.224 \mathrm{lb}, 1 \mathrm{~mm}=0.0394 \mathrm{in})
$$

Figure 7.7 shows typical load-displacement curves (two transducers, stroke, and clipon gage) obtained during the mock tests. It is found that all the curves have a smooth and continuous descending portion after the peak load. The test setup was proved to be effective to conduct stable fracture mechanics tests.

The accuracy of two displacement transducers was good enough to capture the data as precisely as $0.005 \mathrm{~mm}$ (0.0002 in), and the readings of those transducers were very close. Due to the limitation of the accuracy of the actuator itself, the reading of the stroke at the beginning was somehow different from that of the transducers. However, the final reading was almost the same as given by two transducers. 


\subsection{Results and Discussions}

\subsubsection{Crack propagation and fracture surface}

Figure 7.8 shows a typical load-MSD curve obtained during the tests. The entire loading process is divided into three different stages. From point $\mathrm{O}$ to point $\mathrm{A}$, the notched beam behaves elastically and no crack was initiated. From point A to point B, with the increase of the load, the beam shows some inelastic features. Theoretically, at this stage, the crack was initiated and started to propagate very slowly. However, no visual crack was detected at the surface of the notch area as illustrated in Figure 7.9 (a), which was the picture taken right after the load reached maximum point B. After the load exceeded the maximum point $B$, the crack length passed its critical value. At this stage, the crack propagated very quickly, and a clear crack propagating path can be observed on the surface of the notch area as shown in Figure 7.9 (b) and Figure 7.9 (c). At point C, the crack has already passed through the entire ligament of the beam, as shown in Figure 7.9 (d), and the beam was assumed to have failed.

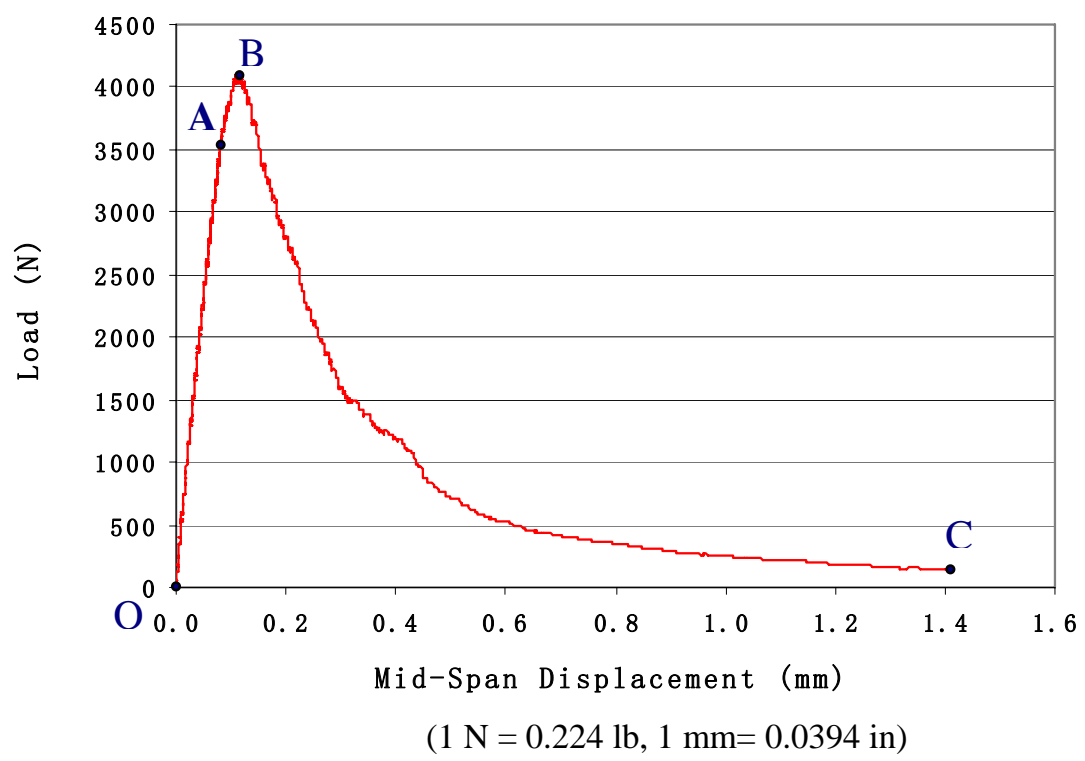

Figure 7.8 Typical load-MSD curve 


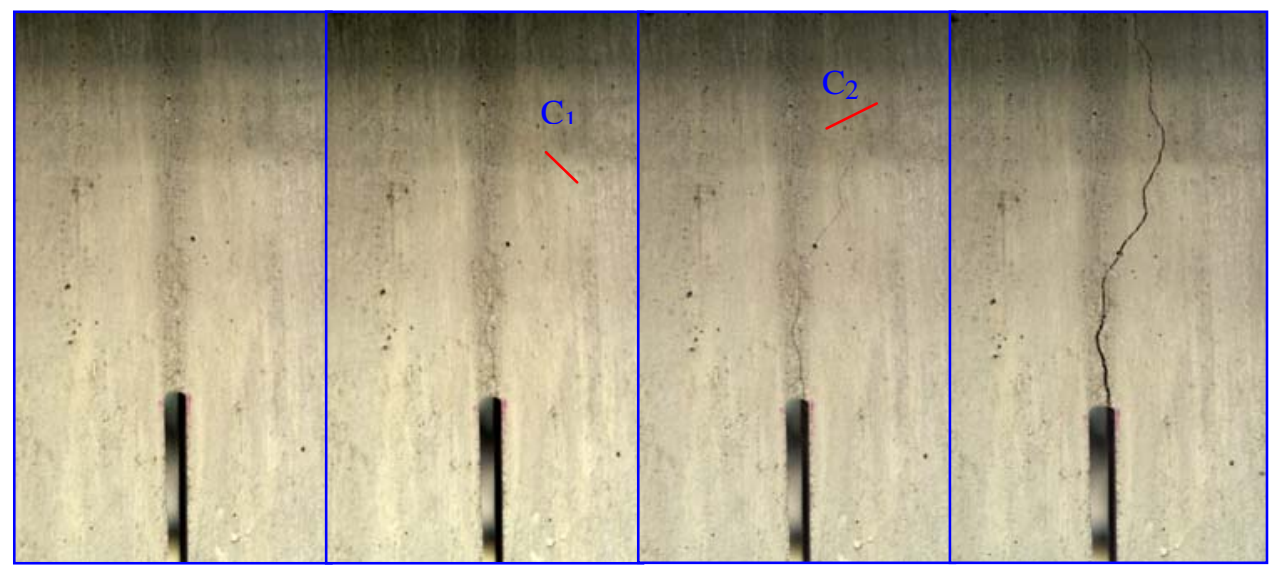

(a)

(b)

(c)

(d)

Figure 7.9 Illustration of the crack propagation

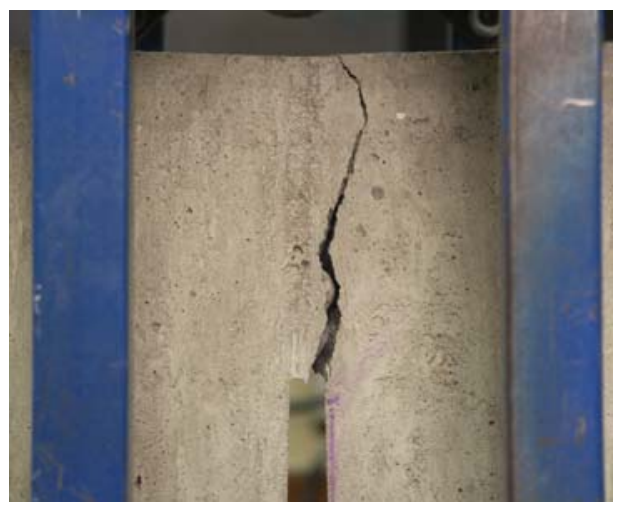

(a)

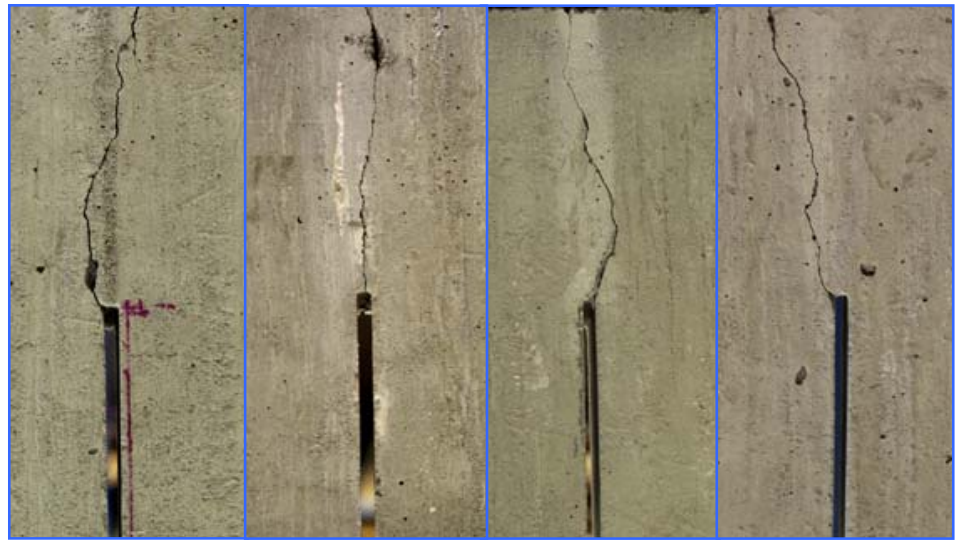

(b)

Figure 7.10 Typical crack propagation paths 


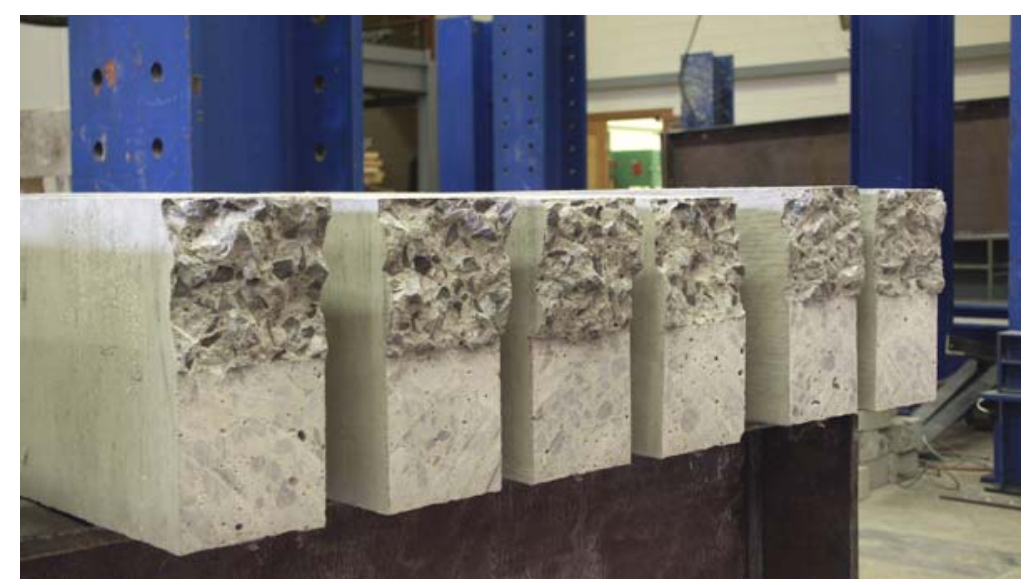

Figure 7.11 The fracture surface of typical broken specimens

Figure 7.10 (a) shows a close view of a fractured specimen, and Figure 7.10 (b) shows typical crack propagation paths. Arbitrary and tortuous crack propagating paths were observed for all the specimens.

Figure 7.11 shows the fracture surfaces of typical broken specimens. Rough fracture surfaces were observed for all the specimens. Figure 7.12 (a), (b), (c), and (d) present close views of the fracture surfaces of the different HPC mixtures at different ages. Figure 7.12 (a) and (b) show the fracture surfaces of the mixture SL-SF-L1-0.4 at the ages of 4-day and 28-day respectively. Figure 7.12 (c) and (d) show the fracture surfaces of the mixture SL-SF-G2-0. at the ages of 4-day and 28-day respectively. It is observed that at the early age (4-day), some of the coarse aggregates were pulled out from the paste, while at 28-day, most of the aggregates were found broken through the crosssection. Consequently, for the specimens of later ages, the failure plane passed through both the coarse aggregates and the cement paste, which resulted in a higher peak loads and work-of-fracture energy during the crack propagations. The detailed analysis of fracture energy of different HPC mixtures is discussed in the next section. 


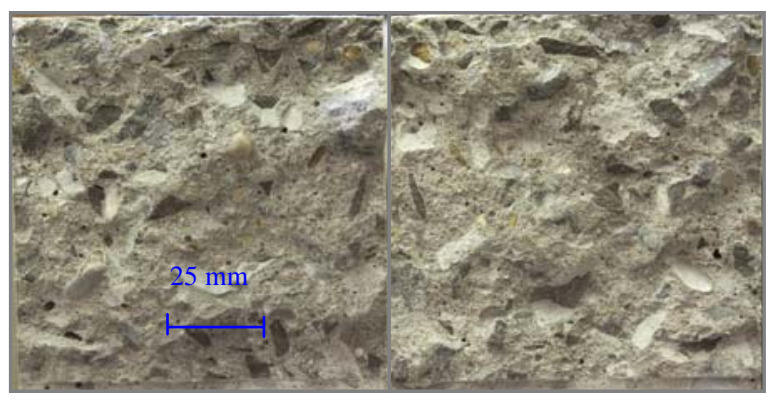

Left

(a)

SL-SF-L1-0.4 (4-day)

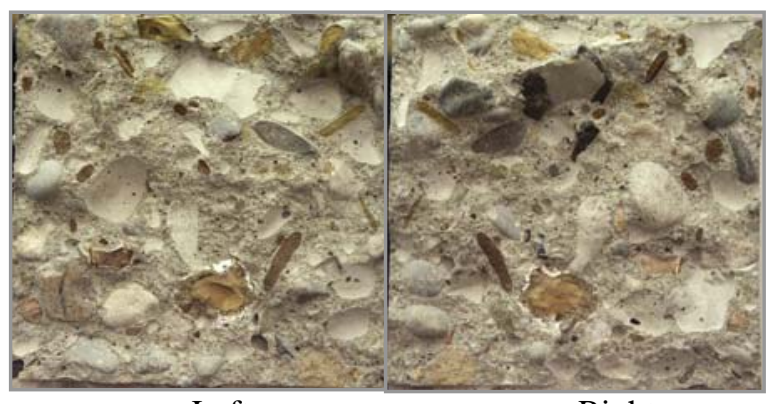

(c)

SL-SF-G2-0.4 (4-day)

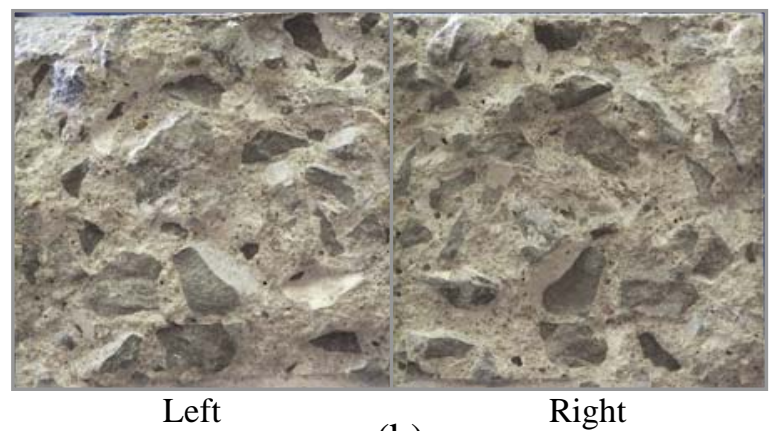

(b)

SL-SF-L1-0.4 (28-day)

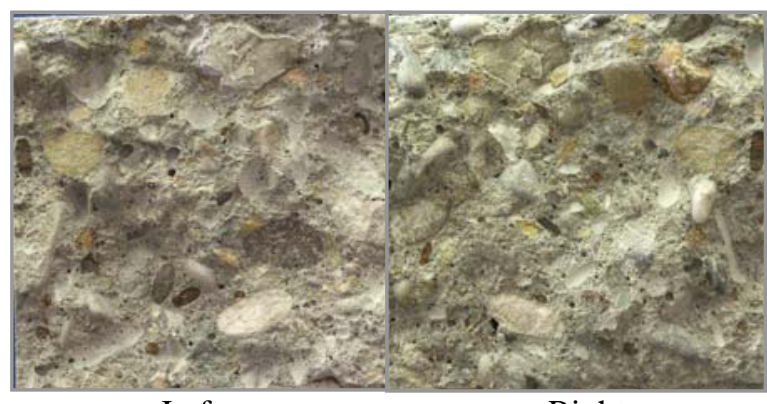

(d)

Figure 7.12 Close views of fracture surfaces

\subsubsection{Determination of the fracture energy of different HPC mixtures}

For each age, three replicate specimens were tested. Figure 7.13 shows the typical plot of load-MSD responses for three replicate specimens at the same age. Some differences were observed especially at the descending stage of the loading; however, there was overall consistency among the specimens tested. 


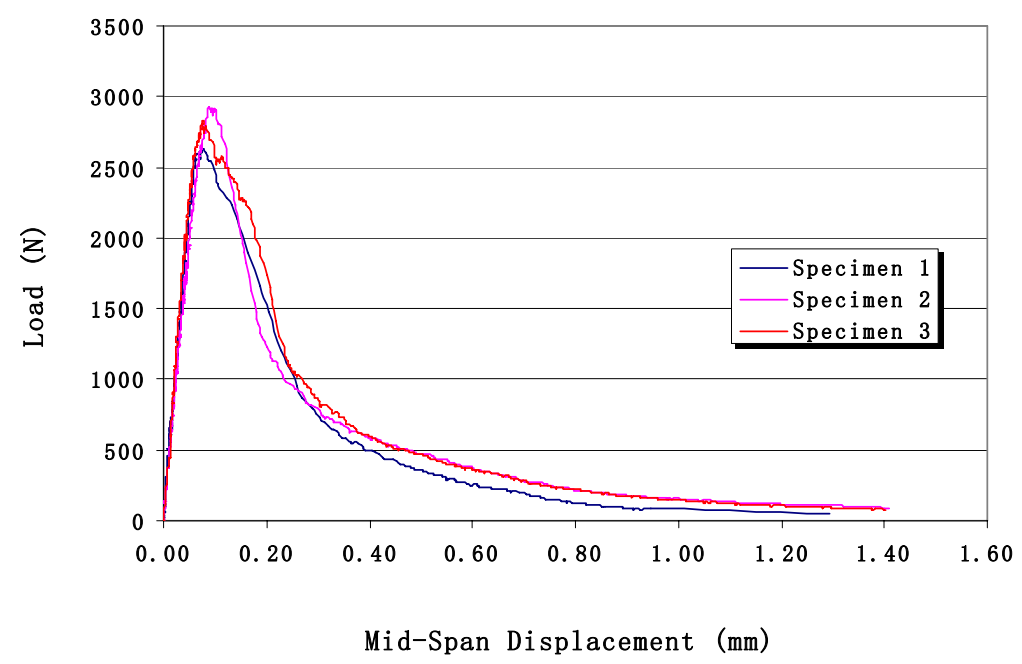

Figure 7.13 Typical load-MSD responses for three replicate specimens

$$
(1 \mathrm{~N}=0.224 \mathrm{lb}, 1 \mathrm{~mm}=0.0394 \mathrm{in})
$$

The determination of fracture energy was performed by following the procedure as recommended by RILEM 50-FMC (1985). However, in this study some modifications were made to achieve a stable and consistent test, as listed below:

1. Partial weight compensation was employed to ensure a stable test. The compensation load comes from the self-weight of cantilever beam.

2. All the tests were stopped whenever the mid-span displacement reached a certain value $(1.40 \mathrm{~mm}$ or $0.0551 \mathrm{in})$ or the load reached a small value (20 $\mathrm{N}$ or $4.5 \mathrm{lbf}$ ) during the descending branch. By doing so, all the tests could be stopped at the same conditions and the uncertainty of sudden failure could be overcome.

As shown in Figure 7.14, the test was stopped at point A. However, theoretically the test should be stopped at point C. According to the study by Petersson (1980), the area of ABC is approximated equal to the product of the load $P_{1}$ and the corresponding 
displacement $\delta$ for the beam with exact weight compensation. It is hereby assumed that the area $S_{2}$ is equal to $P_{1} \delta$ in this study.

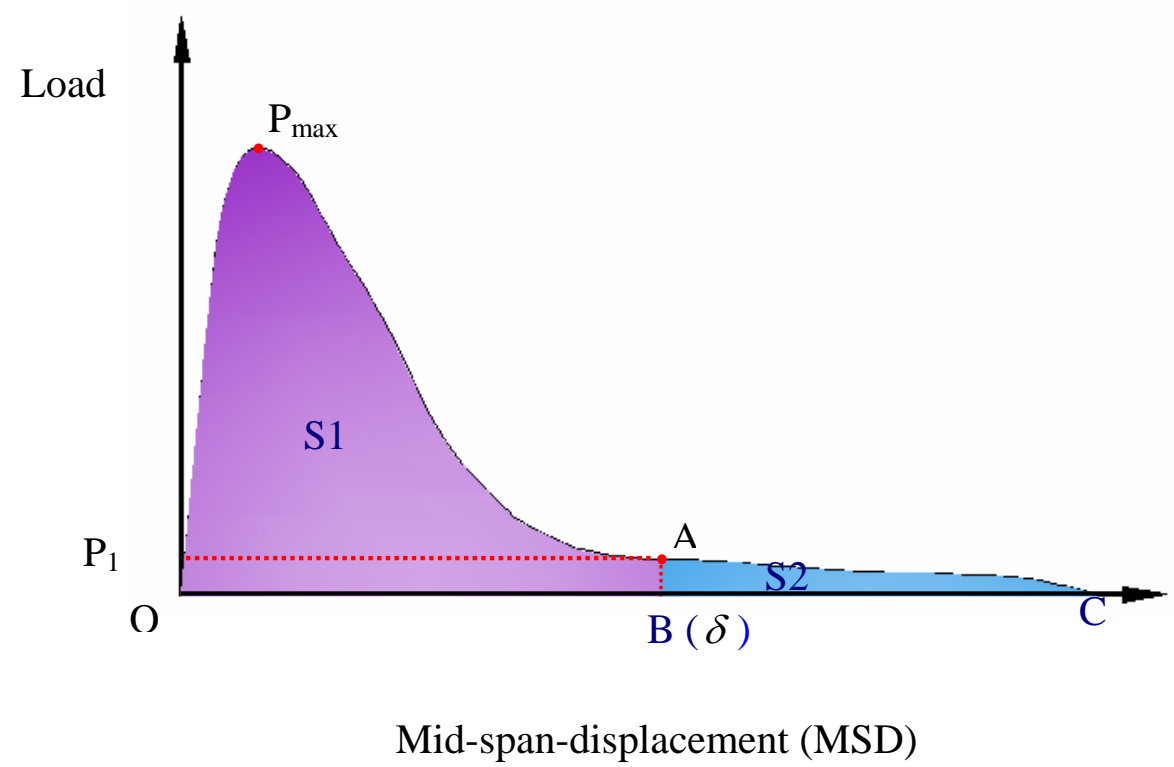

Figure 7.14 The method to calculate the fracture energy

The fracture energy can then be computed as

$$
G_{F}=\frac{S_{1}+S_{2}+m g \delta}{A_{l i g}}
$$

where $G_{F}$ is fracture energy; $S_{1}$ is the area under the curve of load-displacement measured from the test, (as shown in Figure 7.14); $S_{2}=P_{1} \delta, P_{1}$ is the load at the time the test was stopped, and $\delta$ is the corresponding mid-span displacement; $m g$ is the weight ( mass times gravity) of concrete corresponding the mid-span displacement; $A_{\text {lig }}$ is the area of the ligament of the beam. 
The fracture energy based on the effective crack model by Karihallo and Nallathambi (1989) was also computed. The results of both methods are listed in Table 7.1 for comparison of cohesive model approach and effective-elastic model approach. The detailed method of calculation of fracture energy $G_{I C}^{e}$ was discussed in Chapter Two, Section 2.3.3.3 (c).

Table 7.1 Experimental results of fracture energy for different HPC

\begin{tabular}{|c|c|c|c|c|c|c|}
\hline Mixtures & $\begin{array}{l}\text { Ages } \\
\text { (day) }\end{array}$ & $\begin{array}{c}P_{\text {max }} \\
\text { (N) }\end{array}$ & $\begin{array}{c}\delta_{P} \\
(\mathbf{m m})\end{array}$ & $\begin{array}{c}G_{F} \\
(\mathbf{N} / \mathbf{m})\end{array}$ & 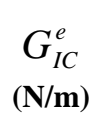 & $G_{F} / G_{I C}^{e}$ \\
\hline \multirow{4}{*}{ SS-L1-0.4 } & 4 & 2840 & 0.069 & 127.3 & 65.7 & 1.94 \\
\hline & 7 & 3042 & 0.080 & 141.5 & 76.3 & 1.85 \\
\hline & 14 & 3301 & 0.083 & 157.6 & 92.9 & 1.70 \\
\hline & 28 & 3608 & 0.096 & 169.1 & 121.6 & 1.39 \\
\hline \multirow{4}{*}{ MK-L1-0.4 } & 4 & 3393 & 0.071 & 134.8 & 77.1 & 1.75 \\
\hline & 7 & 3538 & 0.089 & 155.0 & 93.9 & 1.65 \\
\hline & 14 & 3626 & 0.095 & 166. 2 & 108.8 & 1.53 \\
\hline & 28 & 3958 & 0.106 & 181.2 & 127.2 & 1.42 \\
\hline \multirow{4}{*}{ SS-L1-0.3 } & 4 & 3299 & 0.091 & 131.2 & 81.7 & 1. 48 \\
\hline & 7 & 3558 & 0.094 & 153.9 & 90.9 & 1.25 \\
\hline & 14 & 3890 & 0.099 & 165.3 & 103.5 & 1.36 \\
\hline & 28 & 4107 & 0.107 & 173.6 & 126.8 & 1.32 \\
\hline \multirow{4}{*}{ MK-L1-0.3 } & 4 & 3984 & 0.087 & 158.9 & 93.7 & 1.49 \\
\hline & 7 & 4195 & 0.082 & 162.7 & 111.1 & 1.31 \\
\hline & 14 & 4129 & 0.089 & 172.8 & 126.5 & 1.34 \\
\hline & 28 & 4549 & 0.109 & 185.3 & 133.6 & 1.38 \\
\hline \multirow{4}{*}{ SS-G2-0.4 } & 4 & 2799 & 0.080 & 117.4 & 79.1 & 1.61 \\
\hline & 7 & 3130 & 0.095 & 137.4 & 109.6 & 1.69 \\
\hline & 14 & 3420 & 0.090 & 164.3 & 120.7 & 1.60 \\
\hline & 28 & 3862 & 0.096 & 177.2 & 134.6 & 1.37 \\
\hline \multirow{4}{*}{ MK-G2-0.4 } & 4 & 3155 & 0.098 & 122.5 & 82.9 & 1.70 \\
\hline & 7 & 3650 & 0.103 & 140.4 & 107.5 & 1.46 \\
\hline & 14 & 3665 & 0.086 & 171.2 & 127.9 & 1.37 \\
\hline & 28 & 3992 & 0.109 & 184.3 & 133.5 & 1.39 \\
\hline
\end{tabular}

$(1 \mathrm{~N} / \mathrm{m}=0.068 \mathrm{lbf} / \mathrm{ft}, 1 \mathrm{~mm}=0.0394 \mathrm{in})$

Note: 1 . The data in the table are the average value of the three replicate specimens.

2. $P_{\max }$ and $\delta_{p}$ are maximum load and corresponding displacement, respectively. 
From Table 7.1, it is apparent that the fracture energy value calculated by RILEM 50 FMC method based on the Hillerborg's cohesive model is always greater than the value calculated by the effective method based on Karihallo's model. The reasons for this difference are below:

Over the last two decades, a number of fractures mechanics tests have been conducted by different researchers. Most of those tests were based on RILEM 50 FMC method. Some of them were also based on the effective elastic methods such as: SEM, TPM or ECM. Due to the theoretical similarities among the effective elastic crack models, the fracture parameters based on different effective elastic models are believed to be comparable. Karihallo and Nallathambi in 1990 and 1991 conducted comparable studies of fracture parameters obtained by SEM, TPM as well as by their own method (ECM). A good agreement was found between $G_{I C}^{e}$ by ECM and $G_{I C}^{s}$ by TPM as well as $G_{I C}^{e}$ by ECM and $G_{f}$ by SEM. However, due to the difference in theoretical approaches and size effect, it is difficult to obtain good correlations between cohesive models and effective crack models. With the assumption of bi-linear softening curves for Hillerborg's test, Planas and Elices in 1990 and 1992 found that the fracture energy was related to $f_{t}$ and $w_{1}$ by equation:

$$
G_{F}=0.86 f_{t} w_{1}
$$

where: $f_{t}$ is tensile strength, and $w_{1}$ is the horizontal intercept of the softening curve (see $w_{c}$ in Fig.2.15). An approximate relation was also given for the parameter $G_{f}$ (SEM), which can be expressed as:

$$
G_{f} \approx 0.45 f_{t} w_{1}
$$


Therefore, the value of $G_{F}$ (cohesive model) in Eq. (7.2) is approximately twice as great as $G_{f}$ obtained from SEM (effective elastic model) in Eq. (7.3). Figure 7.15 shows the relationship between $G_{F}$ and $G_{I C}^{e}$ obtained from tests in this study. The $G_{F}$ value is about 1.25 1.95 times greater than $G_{I C}^{e}$.

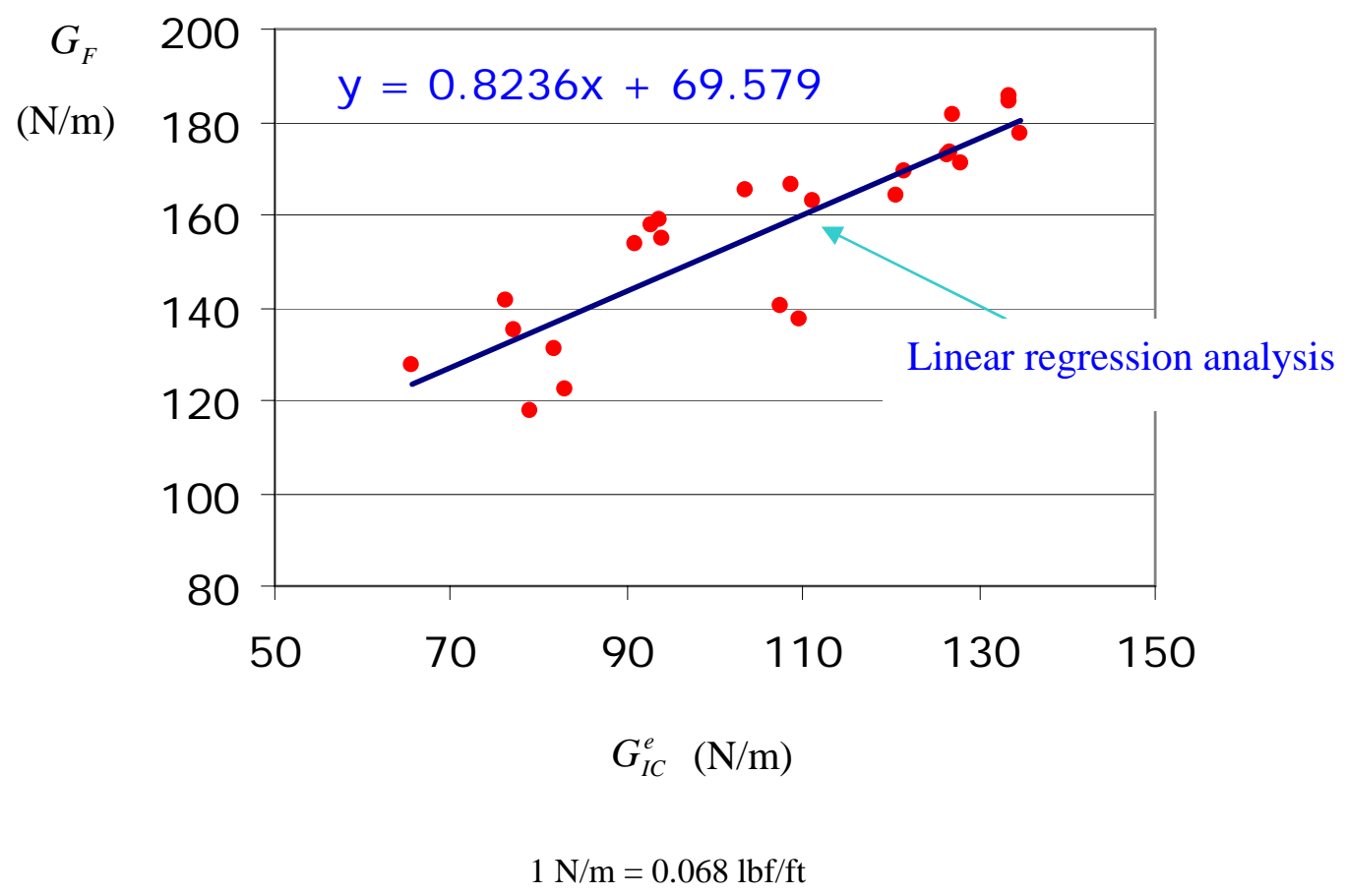

Figure 7.15 Relationship between $G_{F}$ and $G_{I C}^{e}$ 


\subsubsection{Comparison of fracture energy for different HPC mixtures}

\subsubsection{Effect of age}

Figure 7.16 shows fracture energy $G_{F}$ at different ages for all HPC mixtures tested in this study. It is observed that the fracture energy increased with the increase in ages. However, the magnitude of increase is different among mixtures. An increase of 33\% was observed for the mixture SL-SF-L1-0.4 from the age of 4-day to 28-day, while for mixture MK-L1-0.3, the increase was only 17\%, but the MK-L1-0.3 had the highest fracture energy at all ages.

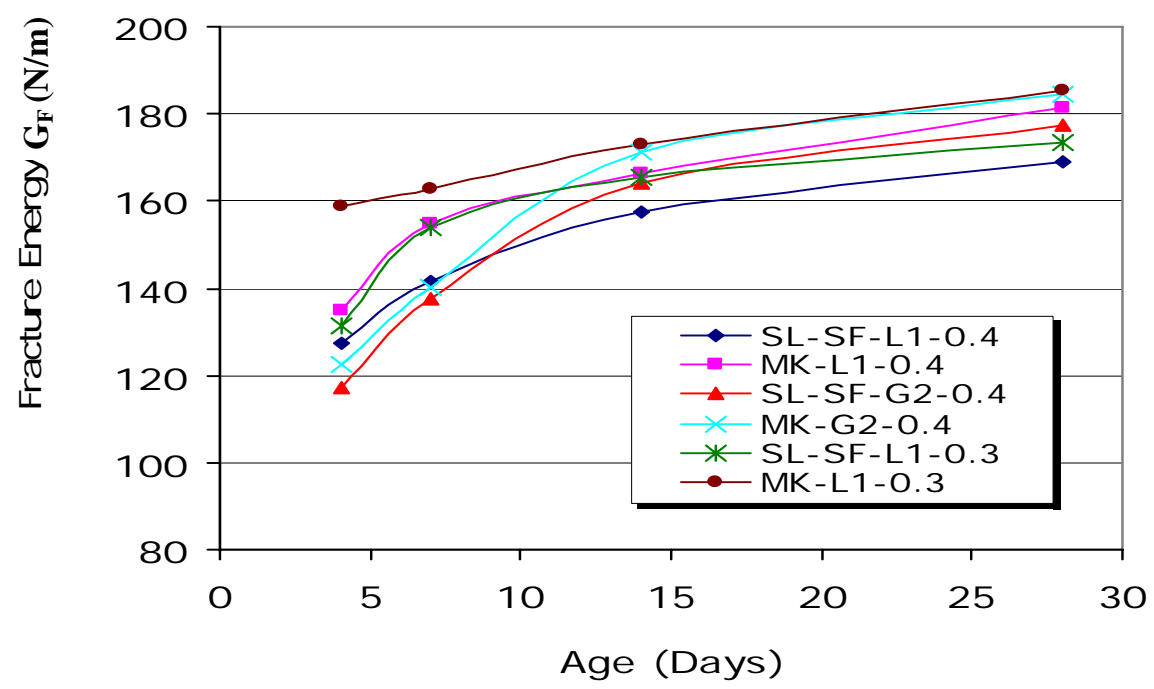

Figure 7.16 Fracture energy with time

\subsubsection{Effect of SCM}

The HPC mixtures selected for fracture mechanics test had two different SCM combinations: one with 35\% slag $+5 \%$ silica fume, the other with $10 \%$ metakaolin. Figure 7.17 shows Load-MSD curves for HPC mixtures with these two SCM 
combinations at the age of 28-day. Figure 7.17 (a) shows mixtures with Limestone 1 and $\mathrm{w} / \mathrm{cm}=0.4$, and Figure 7.17 (b) shows mixtures with gravel 2 and $\mathrm{w} / \mathrm{cm}=0.4$. The loadMSD responses of mixtures with metakaolin covered larger areas compared to mixtures with slag + silica fume for both types of coarse aggregates.

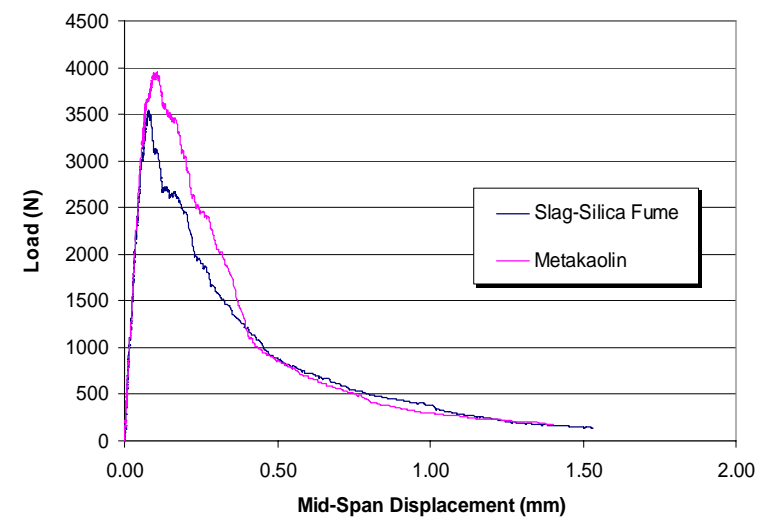

(a) Limestone 1

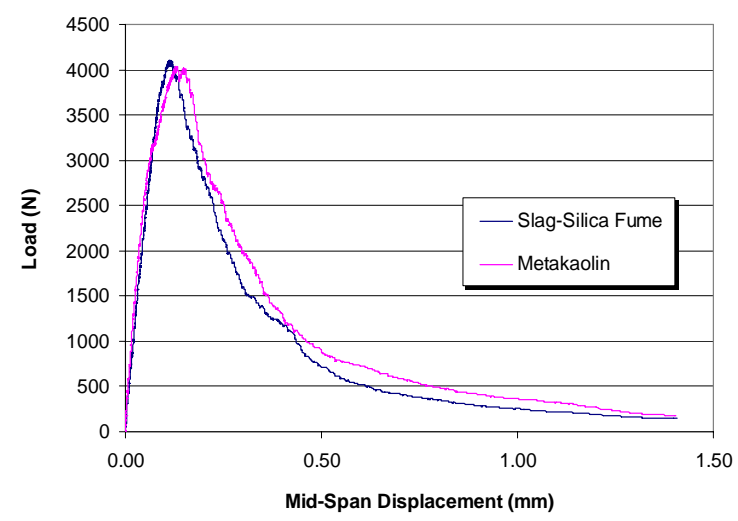

(b) Gravel 2

$(1 \mathrm{~N}=0.224 \mathrm{lbf}, 1 \mathrm{~mm}=0.0394 \mathrm{in})$

Figure 7.17 Load-MSD curves for HPC mixtures with different SCM

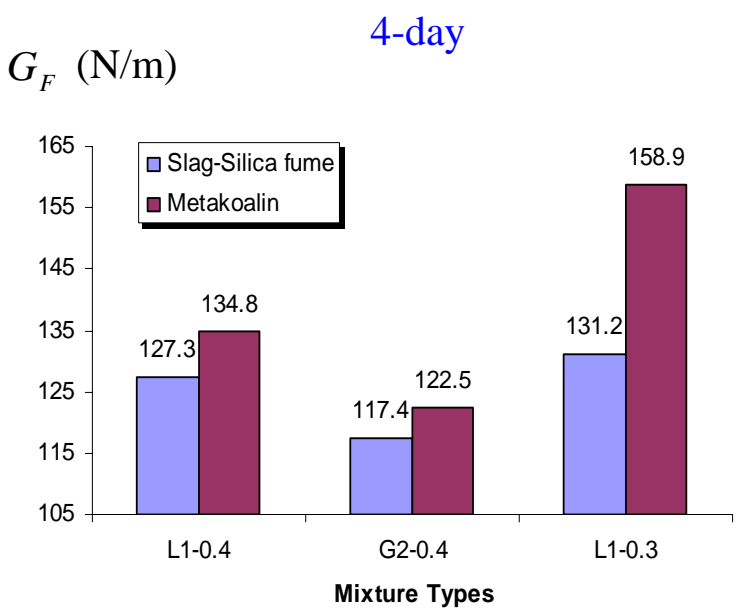

(a)

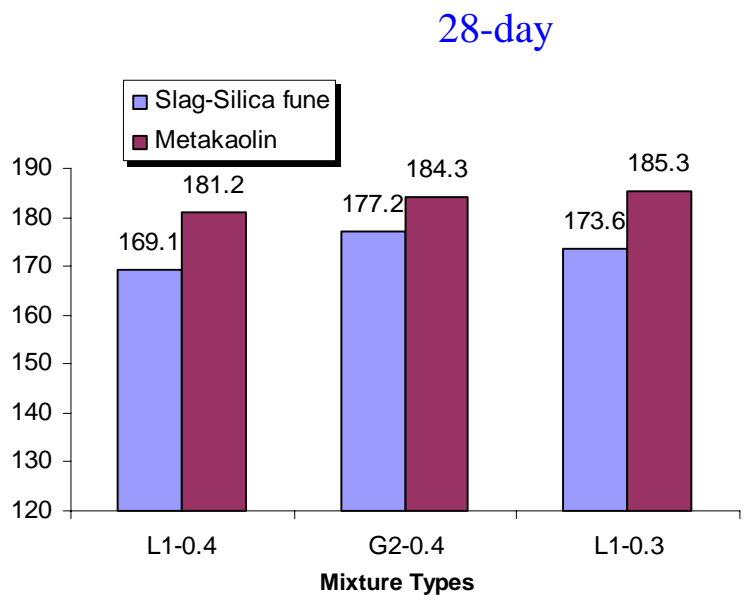

(b)

$(1 \mathrm{~N} / \mathrm{m}=0.068 \mathrm{lbf} / \mathrm{ft}, 1 \mathrm{~mm}=0.0394 \mathrm{in})$

Figure 7.18 Comparison of fracture energy of HPC mixtures with different SCM 
Figure 7.18 (a) and (b) display comparative plots of fracture energy $G_{F}$ for HPC with different SCM at the age of 4-day and 28-day, respectively. Metakaolin HPC shows a higher value of fracture energy compared with HPC with slag + silica fume at the same conditions (the aggregate types and w/cm being the same). From the results of this test as well as the conclusion made in Chapter Four, it is observed that HPC with metakaolin shows a significant improvement in mechanical properties such as compressive strength and fracture energy. This difference was found to be significantly higher in case of w/cm $=0.3$ at the age of 4-day.

\subsubsection{Effect of coarse aggregate types}

Two types of aggregates were used in this study: limestone1 and gravel 2. Figure 7.19 (a) and (b) show load-MSD curves for HPC with slag + silica fume, and w/cm $=0.4$, but different coarse aggregates at the age of 4-day and 28-day, respectively. The HPC mixtures with gravel usually exhibited larger displacement at peak load compared to limestone mixtures. At early age (Figure 7.19 a), the area of load -MSD was smaller in gravel HPC than HPC with limestone, while, at 28-day (Figure 7.19 b), the area covered by gravel HPC became slightly larger than limestone HPC.

Figure 7.20 (a) and (b) display comparative plots of fracture energy for HPC with different coarse aggregates at the age of 4-day and 28-day, respectively. The fracture energy of gravel HPC was lower at early age, but it increased at a higher rate than the limestone HPC. The $G_{F}$ value for gravel HPC was higher than limestone HPC at 28-day for both mixtures. The higher fracture energy and lower modulus of elasticity make gravel HPC less crack prone compared to limestone HPC, and gravel HPC may be a better choice for bridge deck applications. 


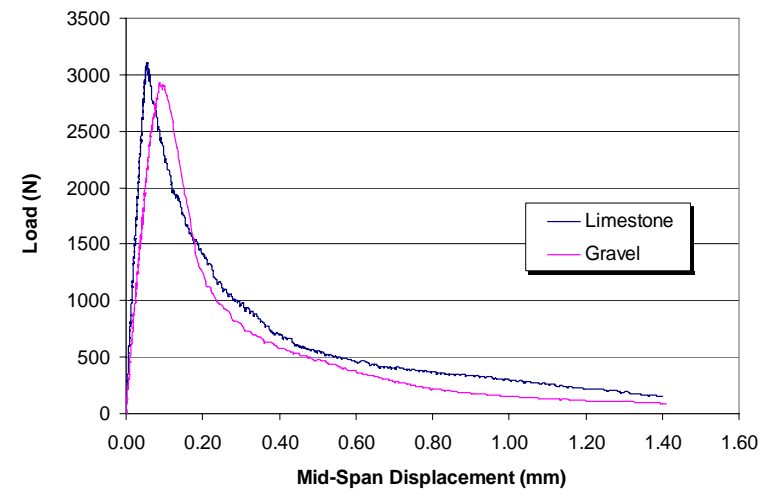

(a) 4-day

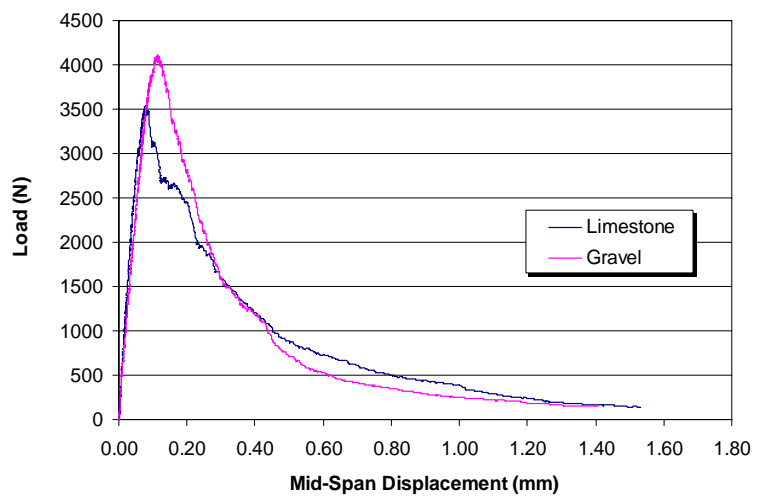

(b) 28-day

(1 $\mathrm{N}=0.224 \mathrm{lbf}, 1 \mathrm{~mm}=0.0394 \mathrm{in})$

Figure 7.19 Load-MSD curves for HPC mixtures with different coarse aggregates

$G_{F}(\mathrm{~N} / \mathrm{m})$

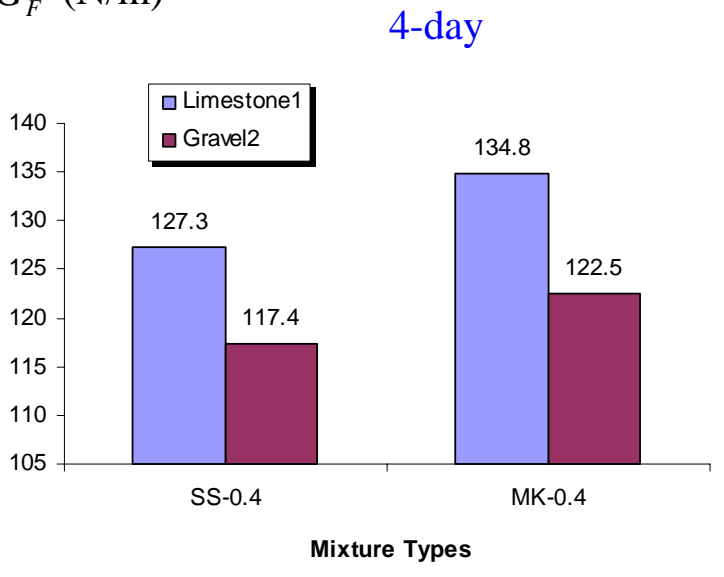

(a)

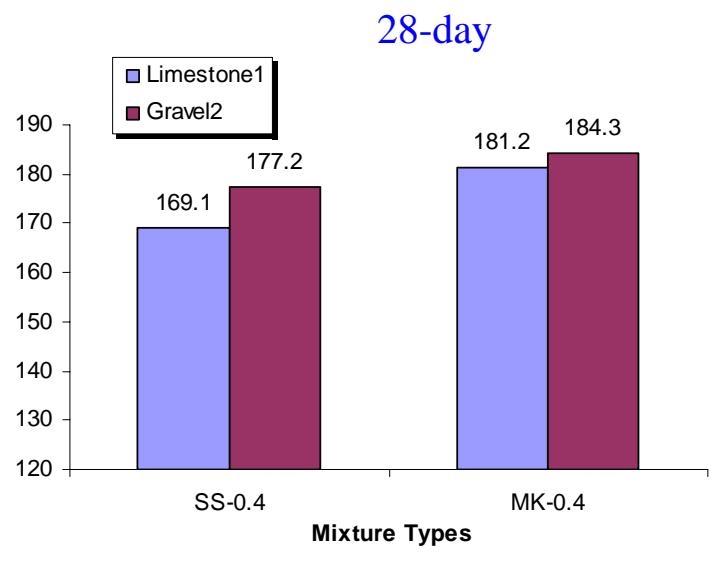

(b)

$(1 \mathrm{~N} / \mathrm{m}=0.068 \mathrm{lbf} / \mathrm{ft}, 1 \mathrm{~mm}=0.0394 \mathrm{in})$

Figure 7.20 Comparison of fracture energy for HPC mixtures with different coarse aggregates

\subsubsection{Effect of w/cm}

Figure 7.21 (a) and (b) show load-MSD curves for HPC mixtures with metakaolin and limestone 1 , and two different $\mathrm{w} / \mathrm{cm}(\mathrm{w} / \mathrm{cm}=0.4$ and $\mathrm{w} / \mathrm{cm}=0.3)$ at the age of 7-day 
and 28-day, respectively. HPC with $\mathrm{w} / \mathrm{cm}=0.3$ shows higher maximum load at all ages, however the area covered by load-MSD curves did not show much difference, especially at 28-day. Also HPC with w/cm = 0.3 had a steeper softening curve compared to HPC with $\mathrm{w} / \mathrm{cm}=0.4$.

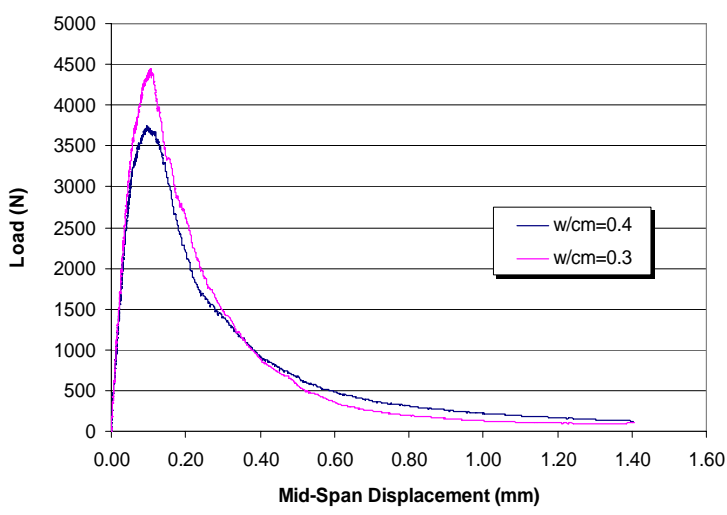

(a) 7-day

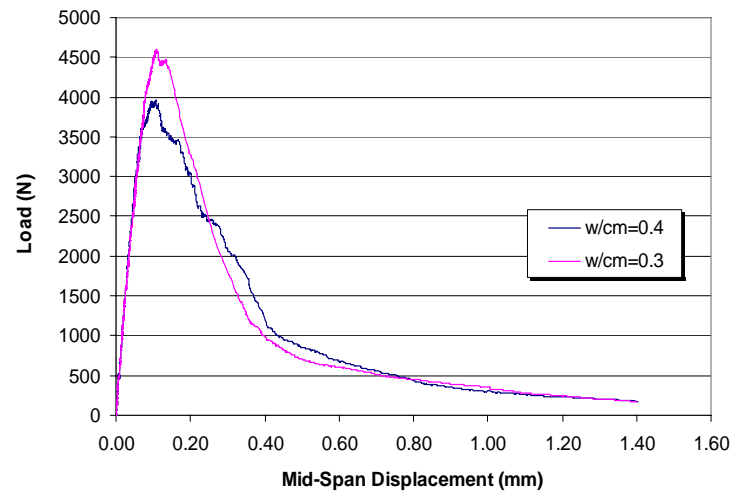

(b) 28 -day

(1 $\mathrm{N}=0.224 \mathrm{lb}, 1 \mathrm{~mm}=0.0394 \mathrm{in})$

Figure 7.21 Load-MSD curves for HPC mixtures with different w/cm

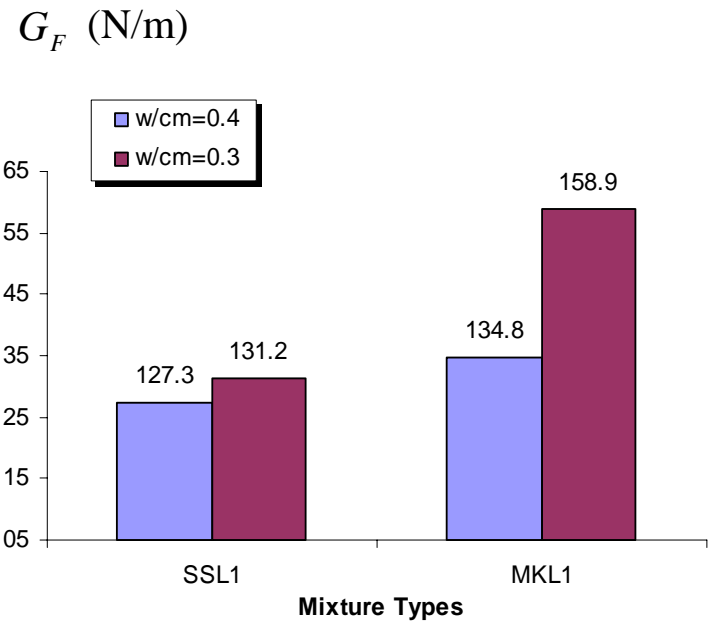

(a)

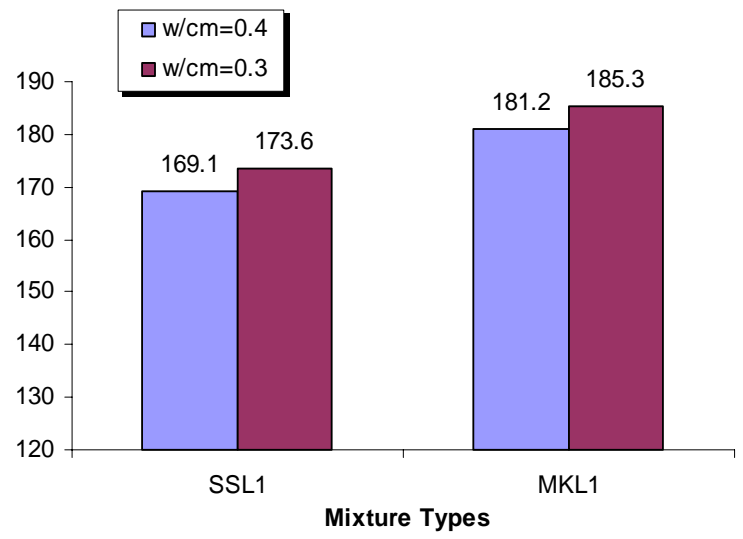

(b)

$(1 \mathrm{~N} / \mathrm{m}=0.068 \mathrm{lbf} / \mathrm{ft}, 1 \mathrm{~mm}=0.0394 \mathrm{in})$

Figure 7.22 Comparison of fracture energy for HPC mixtures with different w/cm 
Figure 7.22 (a) and (b) display comparative plots of fracture energy $G_{F}$ for HPC mixtures with two different w/cm $(\mathrm{w} / \mathrm{c}=0.4, \mathrm{w} / \mathrm{c}=0.3)$ at the age of 4-day and 28-day, respectively. It is observed that HPC with a lower w/cm $(\mathrm{w} / \mathrm{c}=0.3)$ showed a higher fracture energy value for both ages, but the difference is slight at later age (28-day). For instance, the fracture energy of mixture SL-SF-L1-0.3 $\left(G_{F}=173.6 \mathrm{~N} / \mathrm{m}\right)$ is only $2.6 \%$ higher than that $\left(G_{F}=169.1 \mathrm{~N} / \mathrm{mm}\right)$ of mixture SL-SF-L1-0.4.

The fracture energy did not increase proportionately as the w/cm was lowered. Also, as the $\mathrm{w} / \mathrm{cm}$ became lower, the modulus of elasticity and free shrinkage increase (as discussed in Chapter Four). This may increase the potential of cracking for HPC with $\mathrm{w} / \mathrm{cm}$ as low as 0.30 .

\subsubsection{Relationship between fracture energy $G_{F}$ and compressive strength}

Both the fracture energy and compressive strength may be regarded as basic material properties of the concrete. The determination of fracture energy by testing is a difficult process. Any correlations between those two properties may be helpful for the designers.

Figure 7.23 shows the relationship between fracture energy $G_{F}$ and compressive strength for all current HPC mixtures tested at different ages. A general trend of higher values of fracture energy was observed with the increase in compressive strength. In order to establish reliable relationship between fracture energy and compressive strength, more research efforts are needed.

The CEB-FIP Model 90 is the only standard that includes the relationship between fracture energy and compressive strength. The CEB-FIP equation is expressed as:

$$
G_{F}=\left(0.0469 d_{\text {agg }}^{2}-0.5 d_{\text {agg }}+26\right)\left(\frac{f_{c}^{\prime}}{10}\right)^{0.7}
$$


where $G_{F}$ is fracture energy; $d_{a g g}$ is the maximum size of coarse aggregate (in this study, the maximum size of coarse aggregate $d_{\max }=25 \mathrm{~mm}$ ), and $f_{c}^{\prime}$ is mean 28-day compressive strength of concrete in MPa.,

The CEB-FIP prediction is also included in the Figure 7.23. It was observed that the CEB-FIP equation underestimated the fracture energy for HPC.

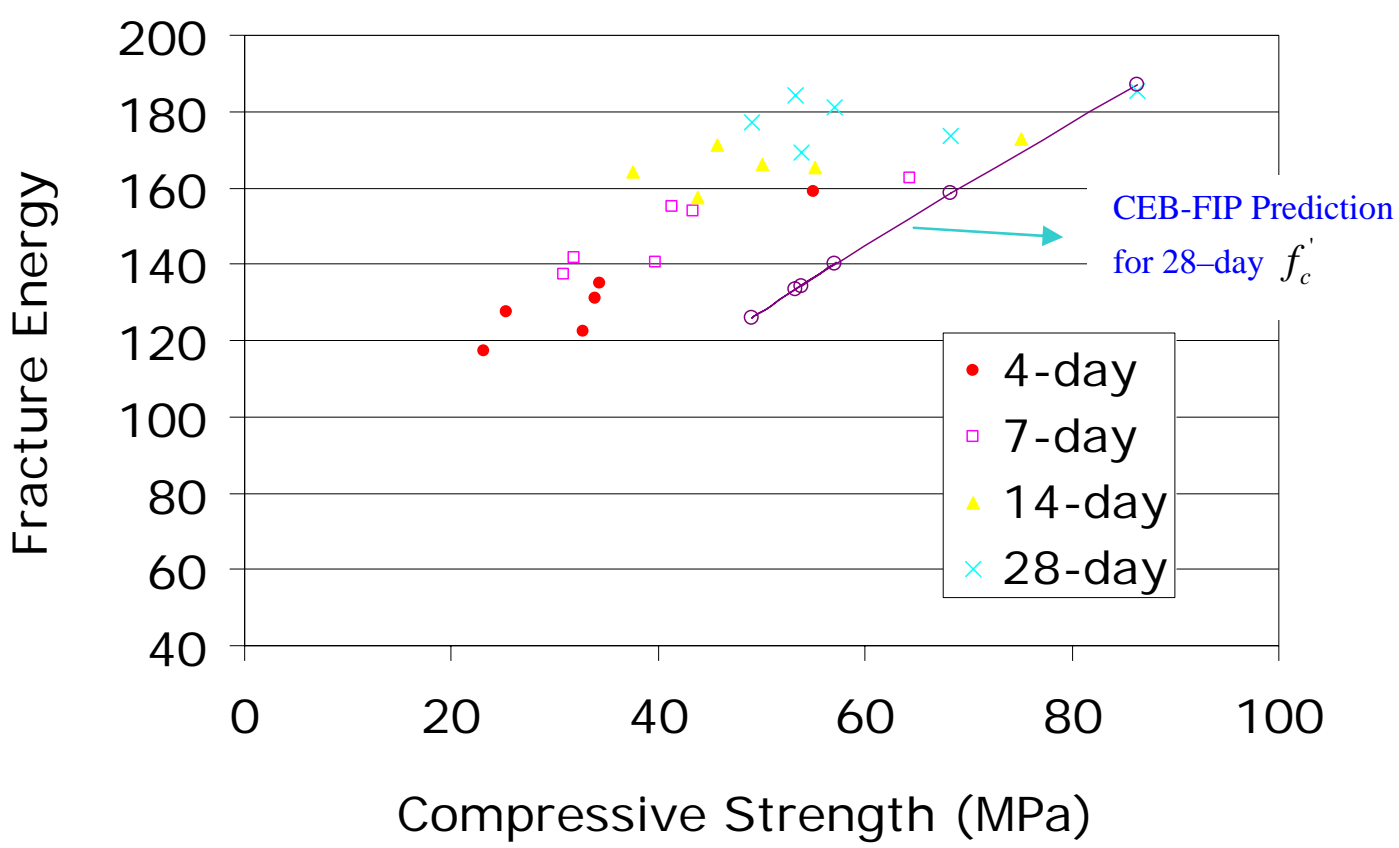

$(1 \mathrm{~N} / \mathrm{m}=0.068 \mathrm{lbf} / \mathrm{ft}, 1 \mathrm{MPa}=145 \mathrm{psi})$

Figure 7.23 Relationship between fracture energy and compressive strength 


\subsubsection{Size effect for fracture mechanics test}

As it has been discussed before, there are several fracture mechanics models for concrete, and each model has its own testing method to determine fracture energy. Different test methods have specimens with different sizes and notch depths. Since fracture energy is also sensitive to size, the different beam size and notch depths generate different testing results even if the other conditions are the same. This size effect was evaluated in this research for HPC.

Table 7.2 Size effect for fracture energy

\begin{tabular}{|c|c|c|c|c|}
\hline $\begin{array}{c}\text { Mixtures } \\
\text { (1) }\end{array}$ & $\begin{array}{c}\text { Age (day) } \\
(2)\end{array}$ & $\begin{array}{c}G_{F 1}(\mathrm{~N} / \mathrm{m}) \\
\text { (RILEM } 50 \mathrm{FCM}) \\
(3)\end{array}$ & $\begin{array}{c}G_{F 2}(\mathrm{~N} / \mathrm{m}) \\
\text { (Sma11er Beam) } \\
(4)\end{array}$ & $\begin{array}{c}G_{F 2} / G_{F 1} \\
(5)\end{array}$ \\
\hline \multirow{4}{*}{ SS-L1-0. 4} & 4 & 127.3 & 145.56 & 1. 14 \\
\hline & 7 & 141.5 & 171.45 & 1. 21 \\
\hline & 14 & 157.6 & 181.98 & 1. 15 \\
\hline & 28 & 169.1 & 209. 34 & 1. 24 \\
\hline \multirow{4}{*}{ SS-G2-0. 4} & 4 & 117.4 & 119.85 & 1. 02 \\
\hline & 7 & 137.4 & 189.56 & 1.38 \\
\hline & 14 & 164.3 & 203. 69 & 1. 24 \\
\hline & 28 & 177.2 & 240.88 & 1. 36 \\
\hline \multirow{4}{*}{ SS-L1-0. 3} & 4 & 131.2 & 169. 77 & 1.30 \\
\hline & 7 & 153.9 & 199. 27 & 1. 29 \\
\hline & 14 & 165.3 & 211.75 & 1. 28 \\
\hline & 28 & 173.6 & 226.45 & 1.30 \\
\hline
\end{tabular}

$(1 \mathrm{~N} / \mathrm{m}=0.068 \mathrm{lbf} / \mathrm{ft})$

Table 7.2 lists the fracture energy values obtained from two separate tests. The $3^{\text {rd }}$ column in Table 7.2 is the results from test following the RILEM 50 FCM. As discussed previously, the beam in this test was $1200 \mathrm{~mm}$ (47 in) long, $200 \mathrm{~mm}$ (8 in) deep, and the notch-to-depth ratio was 0.5 . The $4^{\text {th }}$ column is the results of the test designed to 
construct the R-curve for ring specimens. As discussed in Chapter Six, the beam was 483 mm (19 in) long, $102 \mathrm{~mm}$ (4 in) deep, and the notch-to-depth ratio was only 0.1. The span-to-depth ratios for both tests were 4. From Table 7.2, it is obvious that the fracture energy from smaller specimens is always larger than that from RILEM 50 FCM specimens (bigger specimens). The ratios $\left(G_{F 2} / G_{F 1}\right)$ varied from 1.02 to 1.38 .

\subsection{Concluding Remarks}

1. The rigid frame and loading system set up for this study was able to achieve stable fracture mechanics tests.

2. Fracture surfaces show that for the specimens at early ages (4-day), some of the coarse aggregates were pulled out from the paste; while for specimens at later ages, the failure plane passed through both the coarse aggregates and the cement matrix.

3. Fracture energy $G_{F}$ increased with the increase in ages. However, the magnitude of increase was different among mixtures. The MK-L1-0.3 had the highest fracture energy at all ages.

4. Metakaolin HPC shows a higher value of fracture energy compared with HPC with slag + silica fume at the same conditions.

5. Fracture energy of gravel HPC was lower at the early age, but it increased at a higher rate than the limestone HPC, and the 28-day $G_{F}$ value was higher than limestone HPC.

6. Fracture energy did not increase proportionately as the w/cm became lower.

7. Under the same conditions, the fracture energy values based on cohesive model were always higher than those based on effective crack model. In this study, the $G_{F}$ values 
obtained by the fictitious crack model (FCM) were about $1.30 \sim 1.95$ times greater than $G_{I c}^{e}$ values obtained by the effective crack model (ECM).

8. The fracture energy increased with the increase in compressive strength. More research work is needed for a reliable relationship between fracture energy and compressive strength of different HPC mixtures.

9. Size effect of fracture mechanics test of beams with different sizes and notch depths was evaluated in this study. It was found that the fracture energy from smaller size specimens was always larger than that from larger specimens for the same concrete material. 


\section{CHAPTER EIGHT}

\section{CONCLUSIONS AND RECOMMENDATIONS}

As part of the research project "High Performance Concrete for Bridge Deck Applications," this study is focused on the cracking properties of different HPC mixtures developed using locally available materials. The mechanical properties such as compressive strength, direct tensile strength, modulus of elasticity, as well as free shrinkage and restrained shrinkage of differenet HPC mixtures were studied. A cracking index concept was proposed as an effective method to evaluate the cracking property of different HPC mixtures. A numerical analysis procedure was developed for the AASHTO ring test. A comprehensive fracture mechanics tests study was conducted to obtain the fracture energy for selected HPC mixtures. The detailed concluding remarks are included at the end of each chapter. In this chapter, generalized conclusions are presented based on entire work, and recommendations for future work are also given.

\subsection{Conclusions}

\subsubsection{Hardened properties of HPC}

HPC with metakaolin exhibited the highest compressive strength among all SCM combinations; HPC with ternary combination (slag + fly ash + silica fume) showed lowest compressive strength. Compressive strength increased with the decrease in w/cm at all ages.

For all HPC tested, the direct tensile strength increased rapidly up to 14-day and then the increase became gradual due to the development of microcracks for air curing of specimens. The 90-day strength was slightly lower than the 28-day strength due to the so 
called "strength retrogression.” A relationship between direct tensile strength and compressive strength was proposed on the basis of the test results from this study. This relationship was compared with relationships proposed by other researchers and codes.

Tensile modulus of elasticity was determined by a procedure which considered the influence of high stress. This tensile modulus of elasticity was used to analyze the cracking of the ring specimens. The 28-day compressive modulus of elasticity was also obtained for all HPC mixtures. The modulus of elasticity (both tensile and compressive) of HPC with limestone was greater than that with gravel under the same conditions. This indicated that gravel HPC had advantage over limestone HPC for cracking resistance.

A correlation between compressive modulus of elasticity and 28-day compressive strength of HPC with local source of aggregates was proposed. The relationships between tensile strength and tensile modulus of elasticity were also proposed.

The HPC with gravel had slightly lower free shrinkage compared to HPC with limestone. HPC with SCM (except HPC with Silica fume) had a lower free shrinkage compared to control concrete (with same cementitious material content). HPC with metakaolin exhibited the best performance for free shrinkage. The current free shrinkage prediction models underestimated free shrinkage for HPC at early ages. A prediction model on the basis of regression analysis was proposed. This model can be used to estimate the free shrinkage values of HPC at early age. 


\subsubsection{Restrained shrinkage test}

The AASHTO ring instrumented with strain gauges can capture the cracking of HPC with reasonable accuracy, and proved to be an effective method to evaluate the cracking properties of different HPC. Tests results showed that HPC with gravel generally cracked later than HPC with limestone under the same conditions; for HPC with different SCM combinations, only slight difference was found on the cracking onset. For HPC with lower w/cm, the crack occurred earlier. It can be concluded that higher cementitious materials and lower w/cm made HPC more susceptible to cracking, though the chloride permeability values (not within the scope of this study) can be significantly reduced. It is necessary to define a balance between permeability requirement and cracking requirement when designing HPC mixtures for bridge deck.

A simplified approach (so called cracking index), based on most common material parameters such as compressive strength, modulus of elasticity, and free shrinkage, was recommended to evaluate cracking properties of different HPC mixtures. Real-life field data such as compressive strength and free shrinkage of HPC mixtures from well performing bridge decks in WV were obtained. Based on this data set, the cracking index was estimated, and a threshold cracking onset day was proposed as the criterion for any acceptable HPC mix design with gravel.

The elastic analysis of the ring specimen showed that at onset of cracking, the maximum stress inside concrete ring was lower than the tensile strength of the concrete material. This indicated that the conventional strength limit method is generally not suitable for the failure analysis of ring specimens. 


\subsubsection{Numerical analysis}

Fracture mechanics is an advanced technique for the analysis of cracking related problems. A numerical procedure based on fracture mechanics was developed for predicting the cracking of the AASHTO ring specimen due to the restrained shrinkage. The predicted cracking onset day is reasonably in agreement with the experimental result. This numerical model provided a theoretical basis for the AASHTO ring test. From the experimental as well as the numerical analysis results, it can be concluded that the AASHTO ring test is an effective method for the cracking evaluation of different HPC mixtures.

Based on the G and R curves developed for different HPC mixtures, it was found that the critical crack length $a_{c}$ ranged from $19.9 \mathrm{~mm}$ to $35.6 \mathrm{~mm}$. The variations in $a_{c}$ due to concrete ages are not definite, but there is trend of increasing $a_{c}$ with age with some inconsistencies. However, for very early age concrete (4-day), the $a_{c}$ was always lowest compared with other ages. At early age, the maximum tensile strains from fracture mechanics approach were lower than those from direct tensile tests, while at later ages, the values were higher compared to those from direct tests.

\subsubsection{Fracture mechanics test}

Fracture energy $G_{F}$ increased with the increase in ages. However, the magnitude of increase was different among mixtures. For HPC with lower w/cm $(\mathrm{w} / \mathrm{cm}=0.3)$, fracture energy increasing rate was slower than that of HPC with higher w/cm $(\mathrm{w} / \mathrm{cm}=0.4)$. Metakaolin HPC showed a higher value of fracture energy compared with HPC with slag + silica fume under the same conditions. Fracture energy of gravel HPC was lower at the 
early age, but increased at a much higher rate than the limestone HPC, and eventually, the $G_{F}$ value for gravel HPC at 28-day became higher than for limestone HPC, thus, it can be concluded that HPC mix designed with gravel and metakaolin and a medium w/cm (0.4) may be the best choice for bridge decks since this mixture has a good balance among strength, permeability and cracking (modulus, free shrinkage, and fracture energy). HPC with w/cm $=0.35$ may also be an alternative because of its good strength, very low permeability, and acceptable cracking potential.

Under the same conditions, the fracture energy values based on cohesive model are always higher than those based on effective crack model. In this study, the $G_{F}$ values obtained by FCM were about $1.30 \sim 1.95$ times greater than $G_{I c}^{e}$ values obtained by ECM.

The fracture energy increased with the increase in compressive strength. Comparing current research with CEB-FIP correlation between fracture energy and compressive strength, it was found that for the HPC with the strength less than $60 \mathrm{MPa}$ (8700 psi), the CEB-FIP equation underestimated the fracture energy value; while at a compressive strength larger than $60 \mathrm{MPa}$ (8700 psi), the CEB-FIP equation overestimated the actual fracture energy value.

Size effect of fracture mechanics tests of beams with different sizes and notch depths was evaluated in this study. It was found that the fracture energy from smaller size specimens was always larger than that from larger specimens for same concrete material. 


\subsection{Recommendations}

Although a relatively comprehensive study on cracking of different HPC is provided in this dissertation, further investigations are suggested in the following areas.

1. In order to establish a more reliable relationship between cracking index $C_{\text {ind }}$ and basic parameters (28-day compressive strength and modulus of elasticity, 90-day free shrinkage), more experimental work is needed in the future, which should cover a wider range of strength, aggregate types, and SCM combinations. Meanwhile, more field data from well performing bridge projects statewide is also required in order to better define a threshold value. The field data should include HPC with different types of coarse aggregates.

2. More fracture mechanics tests are recommended for a wider selection of HPC leading to the following achievements:

- A reliable correlation between facture energy and compressive strength of HPC with local material sources could be established.

- The numerical procedure as developed in Chapter Six could be verified by more cases to make the predicting results more consistent.

3. The tensile creep tests of different HPC mixtures are suggested in the future so that Bazant's B3 creep model can be verified or modified for the application to HPC mixtures. 


\section{REFERENCES}

AASHTO T 22 /ASTM C-39, "Standard Test Method for Compressive Strength of Cylindrical Concrete Specimens.”

AASHTO M 43 / ASTM C33, “Standard Specification for Concrete Aggregates.”

AASHTO T 119 / ASTM C 143, “Slump of Hydraulic Cement Concrete.”

AASHTO T 121 / ASTM C 138, "Unit Weight, Yield, and Air Content (Gravimetric) of Concrete."

AASHTO T 126 / ASTM C 192, "Making and Curing Concrete in the Laboratory.”

AASHTO T 152 / ASTM C 231, “Air Content of Freshly Mixed Concrete by the Pressure Method.”

AASHTO T 160 / ASTM C 157, “Test for Length Change of Hardened Cement Mortar and Concrete."

AASHTO Designation PP34-99 "Standard Practice for Estimating the Cracking Tendency of Concrete.”

AASHTO, “LRFD Bridge Design Specification,” 2002.

ACI Committee 209R-92, "Prediction of Creep, Shrinkage, and Temperature Effects in Concrete Structures,” American Concrete Institute, 1992.

ACI-318, "Building Code Requirement for Structural Concrete," American Concrete Institute, 2002.

ACI Committee 363R, "State-of-Art Report on High Strength Concrete," American Concrete Institute, 1997.

ACI Committee 446.1R-91, "Fracture Mechanics of Concrete: Concepts, Models and Determination of Material Properties,” American Concrete Institute, 1989.

Ahmad, S. H., and Shah, S. P., "Structural Properties of High Strength Concrete and its Application for Precast Prestressed Concrete,” PCI Journal, V. 30, No. 6, 1985, pp 92119.

Aitcin, P., “High Performance Concrete,” First Edition, Spon, Aug. 1998.

Anderson, T.L., "Fracture Mechanics: Fundamentals and Applications,” Second Edition, CRC Press, 1995. 
ANSYS 9.0, “Complete User's Manual,” ANSYS Corporation, 2006

ASTM C 260, “Specification for Air-Entraining Admixtures for Concrete.”

ASTM, E 399, "Standard Test Method for Linear-Elastic Plane-Strain Fracture Toughness $K_{I c}$ of Metallic Materials.”

ASTM C 469, "Static Modulus of Elasticity and Poisson's Ratio of Concrete in Compression.”

ASTM C 494, “Specification for Chemical Admixtures for Concrete.”

ASTM C 878, "Standard Test Method for Restrained Expansion of ShrinkageCompensating Concrete.”

Banthia, N.; Yan, C., and Mindess, S., "Restrained Shrinkage Cracking in Fiber Reinforced Concrete: a Novel Test Technique," Cement and Concrete Research, V. 26, No. 1, 1996, pp. 9-14.

Barenblatt, G. I., “The Formation of Equilibrium Cracks Formed during Brittle Fracture,” PMM, V. 23, No. 3, 1959, pp. 434-444.

Bazant, Z. P., and Oh, B. H., "Crack Band Theory for Fracture of Concrete," Materials and Structures, V. 16, 1983, pp.155-177.

Bazant Z. P.; Kim, J. K., and Pfeiffer, P. A., "Nonlinear Fracture Properties from Size Effect Tests,” Journal of Structural Engineering, V. 112, No. 2, 1986, pp. 289-307.

Bazant, Z. P., and Prasannan, S., "Solidification Theory for Concrete Creep, I. Formulation and II. Verification and Application,” ASCE, Journal of Engineering Mechanics, V. 115, 1989, pp. 1691-1725.

Bazant, Z. P., and Kazemi, M. T., "Determination of Fracture Energy, Process Zone Length and Brittleness Number from Size Effect, with Application to Rock and Concrete,” International Journal of Fracture, V. 44, 1990, pp. 111-131.

Beacham, M. W., "HPC Bridge Deck in Nebraska," Concrete International, V. 21, No. 2, 1999, pp. 66-68.

Bennett, E. W., and Laot, D. R., "Shrinkage and Creep of Concrete as Affected by Fineness of Portland Cement," Magazine of Concrete Research, V. 22, No. 71, 1970, pp. 69-78.

Bharatkumar, B. H.; Raghuprasad, B. H.; Ramachandramurthy, D. S.; Narayanan, R., and Gopalakrishnan, S., "Effect of Fly Ash and Slag on the Fracture Characteristics of HighPerformance Concrete,” Materials and Structures, V. 38, Jan.-Feb., 2005, pp. 63-72. 
Bissonnette, B.; Marchand, J.; Charron, J. P., and Delagrave, A., "Early Age Behavior of Cement-Based Materials," Materials Science of Concrete VI Proceeding 2001, the American Ceramic Society, 2001, pp. 243-326.

Bloom, R., and Bentur, A., "Free and Restrained Shrinkage of Normal and HighStrength-Concrete,” ACI Material Journal, V. 92, No. 2, 1995, pp. 211-217.

Brooks, J. J., and Megat, J. M. A., "Effect of Metakaolin on Creep and Shrinkage of Concrete,” Cement and Concrete Composite, V. 23, 2001, pp. 495-502.

Caldarone, M. A.; Gruber, K. A.; Burg, R. G., "High Reactivity Metakaolin: a New Generation Mineral Admixture,” Concrete International, V. 16, No. 11, 1994, pp. 37-40.

Carlson, R. W., “Cracking of Concrete,” Boston Society of Civil Engineers, BSCE, Jan. 1942.

Carlson, R. W., and Reading, T. J., "Model Study of Shrinkage Cracking in Concrete Building Wall,” ACI Structural Journal, V. 85, No. 4, 1998, pp. 395-404.

Chatterjee, Ani., "Shrinkage and Strength Characterizations of Concrete Containing Supplementary Cementing Materials,” Thesis (M.S.), West Virginia University, 2004.

Christopher, M. W., “Crack free HPC Bridge Deck --- New Hampshire’s Experience,” HPC Bridge Views, No. 4, July-Aug. 1999.

Coutino, A. S., “The Influence of the Type of Cement on its Cracking Tendency," RILEM Bulletin, No. 5, 1959, pp. 26-40.

Ding, J. T., and Li, Z. J., "Effect of Metakaolin and Silica Fume on Properties of Concrete,” ACI Materials Journal, V. 99, No. 4, 2002, pp. 393-398.

CEB Model Code, Design Code, 1978. pp 12-13.

CEB-FIP Model Code, Design Code, 1990.

Dugdale, D. S., "Yielding of Steel Sheets Containing Slits," Journal of the Mechanics and Physics of Solids, V. 8, 1960.

Elices, M., Planas, J., “The Equivalent Elastic Crack: 1. Load-Y Equivalences,” International Journal of Fracture, V. 61, 1993, pp. 159-172.

Fan, D. Y., "Durability Studies of High Performance Concrete Used for Bridge Deck,” Thesis (M.S.), West Virginia University, 2005.

Federal Highway Administration (FHWA) Annaul Report, 1993. 
Freedman, S., “High Strength Concrete,” ISL-OIT, Portland Cement Association, Skokie, IL, 1971.

French, C.; Eppers, L.; Le, Q. C., and Hajjar, J. F., “Transverse Cracking in Concrete Bridge Deck,” Transportation Research Record, No. 1688, Nov., 1999, pp. 21-29.

Folliard, K. J., and Berke, N. S., " Properties of High-Performance Concrete Containing Shrinkage-Reducing Admixture,” Cement and Concrete Research, V. 27, No. 9,1997, pp. 1357-1364.

Gardner, N. J., and Lockman, M. J., "Design Provisions for Drying Shrinkage and Creep of Normal-Strength Concrete,” ACI Materials Journal, V. 98, No. 2, 2001, pp. 159-167.

Griffith, A. A., “The Phenomenon of Rupture and Flow in Solid," Philosophical Transactions of the Royal Society of London, Series A, V. 221, 1920, pp.163-198.

Grzybowski, M., and Shah, S. P., "Shrinkage Cracking of Fiber Reinforced Concrete," ACI Materials Journal, V. 87, No. 2, 1990, pp.138-148.

Grzybowski, M., and Shah, S. P., "Shrinkage Cracking of Fiber Reinforced Concrete," ACI Materials Journal, V. 87, No. 2, 1990, pp. 138-148.

Goodspeed, C.; Vanikar, S, and Cook, R., "High-Performance Concrete (HPC) Defined for Highway Structures,” Concrete International, V. 18, No. 2, 1996, pp. 62-67.

Hillerborg, A.; Modeer, M., and Petersson, P. E., "Analysis of Crack Formation and Crack Growth in Concrete by Means of Fracture Mechanics and Finite Elements," Cement and Concrete Research, V. 16, No. 6, 1976, pp. 773-782.

Hillerborg, A., "The Theoretical Basis of a Method to Determine the Fracture Energy GF of Concrete,” Materials and Structures, V. 18, No. 106, Jul-Aug, 1985, pp. 291-296.

Huo, X. Sh.; Al-Omaishi, N., and Tadros, M. K., "Creep, Shrinkage, and Modulus of Elasticity of High Performance Concrete,” ACI Materials Journal, V. 98, No. 6, 2001, pp. 440-449.

Igarashi, S.; Bentur, A., and Kovler, K., “Autogenous Shrinkage and Induced Restraining Stress in High-Strength-Concretes,” Cement and Concrete Research, V. 30, 2000, pp. 1701-1707.

Irwin, G. R., "Analysis of Stress and Strains near the End of a Crack Traversing a Plate," Journal of Applied Mechanics, V. 24, 1957, pp. 361-364.

Japan Concrete Institute, JCI Research Report of Autogenous Shrinkage, 1996, pp. 199201. 
Jenq, Y. S., and Shah, S. P., “A Two Parameter Fracture Model for Concrete,” Journal of Engineering Mechanics, V. 111, No. 4, 1985, pp. 1227-1241.

Karihaloo, B. L., and Nallathambi, P, “An Improved Effective Crack Model for the Determination of Fracture Toughness of Concrete," Cement and Concrete Research, V. 19, 1989, pp. 603-610.

Karihaloo, B.L., and Nallathambi, P., "Effective Crack Model for the Determination of Fracture Toughness of Concrete,” Engineering Fracture Mechanics, V. 35, No. 4/5, 1990, pp. 637-645.

Karihaloo, B.L., and Nallathambi, P., "Notched Beam Test: Mode I Fracture Toughness,” Fracture Mechanics Test Methods for Concrete, Shah, S. P. and Carpinteri, A. Editors, Chapman and Hall, 1991, pp. 1-86.

Kerkhoff, B. "Benefits of Air Entrainment in HPC,” HPC Bridge Views, No. 23, Sept.Oct. 2002.

Kjellsen, K.O.; Hallgren, M., and Wallevik, O.H, "Fracture Mechanical Properties of High-Performance Concrete--- Influence of Silica Fume,” Materials and Structures, V. 33, Nov., 2000, pp. 552-558.

Kovler, K., “Testing System for Determining the Mechanical Behavior of Early Age Concrete Under Restrained and Free Uniaxial Shrinkage,” Materials and Structures, V. 27, 1994, pp. 324-330.

Kraai, P. P., "Proposed Test to Determine the Cracking Potential Due to Drying Shrinkage of Concrete,” Concrete Construction, V. 30, No. 9, 1985, pp. 775-778.

Krauss, P. D., and Rogaua, E. A., "Transverse Cracking in Newly Constructed Bridge Decks,” NCHRP Report 380, Transportation Research Board, Washington D.C., 1996.

Li, Q. B., and Ansari F., "High-Strength Concrete in Uniaxial Tension,” ACI Materials Journal, V. 97, No. 1, 2000, pp. 49-57.

Li, Z. j.; Qi, M.; Li, Z. L., and, Ma, B. G., "Crack Width of High-performance Concrete Due to Restrained shrinkage,” Journal of Materials in Civil Engineering, V. 11, No. 3, 1999, pp. 214-223.

Lopez, M.; Kahn, L. F., and Kurtis, K. E., "Creep and Shrinkage of High-Performance Lightweight Concrete,” ACI Materials Journal, V. 101, No. 5, 2004, pp. 391-399.

Lou, j.; Bhalerao, K.; Soboyejo, A. B. O.; Soboyejo, W. O., “An Investigation of Fracture Initiation and Resistance Curve Behavior in Concrete," Cement and Concrete Composites, V. 25, 2003, pp. 599-605. 
Malhotra, V. M., “Concrete Ring for Determining Tensile Strength of Concrete,” ACI Journal, V. 67, No. 4, 1970, pp. 354-357.

Malier, Y., “High Performance Concrete-from Material to Structures”, E\&FN Spon, London, 1992.

McCabe D. E. (Ed.), "Fracture Toughness Evaluation by R-Curve Method,” ASTM- STP 527, ASTM, Philadelphia, PA, 1973.

Mindess, S.; Young, J. F., and Darwin, D., “Concrete,” Second Edition, Prentice Hall, 2003.

Moore, J. A., “AASHTO Lead State Implementation,” HPC Bridge Views, No. 2, March/April, 1999.

Nallathambi, P., and Karihaloo, B. L., "Determination of Specimen-Size Independent Fracture Toughness of Plain Concrete,” Magazine of Concrete Research, V. 38, No. 135, 1986, pp. 67-76.

Neville, A. M., “Properties of Concrete,” Fourth Edition, Wiley, 1996.

Niwa, J., and Tangtermsirikul, S., "Fracture Properties of High Strength and SelfCompacting High-Performance Cocnrete," Transactions of the Japan Concrete Institute, V. 19, 1997, pp.73-80.

Petersson, P. E., "Fracture Energy of Concrete: Practical Performance and Experimental Results,” Cement and Concrete Research, V. 10, 1980, pp. 91-101.

Planas, J., and Elices, M., "Fracture Criteria for Concrete: Mathematical Approximations and Experimental Validation,” Engineering Fracture Mechanics, V. 35, 1990, pp. 87-94.

Planas, J., and Elices, M., "Shrinkage Eigenstresses and Structural Size Effect," International Fracture Mechanics of Concrete Structures, Bazant, P. Z. editor, Eisevier Applied Science, 1992, pp. 939-950.

Quyang C., and Shah S. P., "Geometry-Dependent R-Curve for Quasi-Brittle Materials," Journal of the American Ceramic Society, V. 74, No. 11, 1991, pp. 2831-2836.

Qzyildirim, C., "Laboratory Investigation of Low-Permeability Concrete Containing Slag and Silica Fume,” ACI Materials Journal, V.91, No.2, 1994, pp. 197-203.

Qzyildirim, C., “HPC Bridge Deck in Virginia,” Concrete International, V. 21, No. 2, 1999, pp. 59-60.

Rashid, Y. R., “Analysis of Pretressed Concrete Pressure Vessels,” Nuclear Engineering and Design, V. 7, 1968, pp. 334-344. 
Raus, M. L., “Texas HPC Bridge Deck,” Concrete International, V. 21, No. 2, 1999, pp. 63-65.

Rice, J. R., “A Path Independent Integral and Approximate Analysis of Strain Concentration by Notches and Cracks," Journal of Applied Mechanics, V. 35, 1968, pp. 379-386.

RILEM 50 FMC Committee on Fracture Mechanics of Concrete, "Determination of the Fracture Energy of Mortar and Concrete by Means of Three-Point Bend Tests on Notched Beams,” Materials and Structures, V. 118, No. 106, 1985, pp. 285-290.

RILEM TC-89-FMT, "Determination of Fracture Parameters $\left(K_{I c}^{s}\right.$ and $\left.C T O D_{c}\right)$ of Plain Concrete Using Three Point Bend Tests,” Materials and Structures, V. 23, 1990, pp. 457460.

RILEM TC-89-FMT, "Size Effect Method for Determining Fracture Energy and Process Zone Size of Concrete,” Materials and Structures, V. 23, 1990, pp. 461-465.

RILEM TC Committee TC 107, "Creep and Shrinkage Prediction Model for Analysis and Design of Concrete Structures--- Model B3,” Materials and Structures, V. 28, 1995, pp. 357-365.

Russell, H. G., “ACI Defines High-Performance-Concrete,” Concrete International, V. 21, No. 2, 1999, pp. 56-57.

Saadeghvaziri, M. A., and Hadidi, R., "Cause and Control of Transverse Cracking in Concrete Bridge Deck,” Final Report, Sept. 2002, New Jersey Institute of Technology.

Sakata K.; Tsubaki, T.; Inoue, Sh., and Ayano, T., "Prediction Equations of Creep and Drying Shrinkage for Wide Range Strength Concrete,” Creep, Shrinkage and Durability Mechanics of Concrete and Other Quasi-Brittle Materials, Edited by Ulm,F.J.; Bazant, Z. P., and Wittmam, F.H., Elsevier Science, 2001, pp. 753-756.

See, H. T.; Attiogbe, E. K., and Miltenberger, M. A., "Shrinkage Cracking Characteristics of Concrete Using Ring Specimen,” ACI Materials Journal, V. 100, No. 3, 2003, pp. 239-245.

Shah, S. P.; Quyang, C.; Marikunte, S.; Yang, W., and Becq-Giraudon, E., "Method to Predict Shrinkage Cracking of Concrete,” ACI Materials Journal, V. 95, No. 4, 1998, pp. 339-346.

Somsak, S.; Lu, H. R., and Wee, T. H., "Direct Tension Test and Tensile Strain Capacity of Concrete at Early Age,” Cement and Concrete Research, V. 33, 2003, pp. 2077-2084. 
Springenschmid, R.; Breitenbucher, R., and Mangold, M., "Development of Cracking Frame and the Temperature Stress Testing Machine,” Thermal Cracking in Concrete at Early Age, Edited by Springenschmid, R., E\&FN Spon, London, 1994, pp. 137-144.

Streeter, D. A., "Developing a High-Performance Concrete Mix for New York State Bridge Deck,” Transportation Research Record, 1532, TRB, 1996 pp. 60-65.

Subramaniam, K. V.; Gromotka, R.; Shah, S. P.; Obla K., and Hill, R., "Influence of Ultra-fine Fly Ash on the Early Age Response and the Shrinkage Cracking Potential of Concrete,” Journal of Materials in Civil Engineering, V. 17, No. 1, 2005, pp. 45-53.

Tada, H; Paris, P. C.; Irwin, G. R., “The Stress Analysis of Cracks Handbook,” $3^{\text {rd }}$ Edition, ASME International, New York, 2000.

Timoshenko, S., and Goodier, J. N., “Theory of Elasticity,” McGraw-Hill Education September 30, 1970.

Triandafilou, L. N., "HPC Implementation Status," HPC Bridge Views, No.32, March/April, 2004.

USBR 4914-92, "Procedure for Direct Tension Strength, Static Modulus of Elasticity, and Poisson's Ratio of Cylindrical Concrete Specimens in Tension," Annual Book of ASTM

Wang, Y. J.; Li, V. C., and Backer, S., "Experimental Determination of Tensile Behavior of Fiber Reinforced Concrete,” ACI Materials Journal, V. 87, No. 5, 1990, pp. 461-468.

Wecharatana, M., and Shah, S. P., "Slow Crack Growth in Cement Composites," Journal of Structural Engineering, V. 108, No. 61982 pp.1400-1413.

Weiss, W. J.; Yang, W., and Shah, S. P., "Shrinkage Cracking of Restrained Concrete Slab,” Journal of Engineering Mechanics, V. 124, No. 7, 1998, pp. 765-774.

West Virginia Department of Transportation-Division of Highway (WVDOH), "Strutural Concrete Class H Bridge Decks,” Special Provision, July 1, 2003

Whitting, D. A.; Detwiler, R. J., and Lagergren, E. S., “Cracking Tendency and Drying Shrinkage of Silica Fume Concrete for Bridge Deck Application,” ACI Materials Journal, V. 97, No. 1, 2000, pp. 71-77.

Wiegrink, K.; Marikunte, S., and Shah, S. P., "Shrinkage Cracking of High-StrengthConcrete,” ACI Materials Journal, V. 93, No. 5, 1996, pp. 409-415.

Wild, S.; Khatib, J. M., and Jones, A., "Relative Strength, Pozzolanic Activity and Cement Hydration in Superplasticised Metakaolin Concrete," Cement and Concrete Research, V. 26, No. 10, 1996, pp. 1537-1544. 
Zhang, W.; Kwan, A. K. H., and Lee, P. K. K., “Direct Tension Test of Concrete,” ACI Materials Journal, V. 98, No. 1, 2001, pp. 63-71.

Zia, P.; Leming, M. L., and Ahmad, S. H., "High Performance Concretes, A State-of -the Art Report," Report No. SHRP-C/FR-91-103, Strategic Highway Research Program, National Research Council, Washington D.C., 1991. 


\section{APPENDIX A}

Compressive Strength, Modulus of Elasticity, and Poisson's Ratio for All 24 HPC Mixtures

\begin{tabular}{|c|c|c|c|c|c|c|c|c|c|c|}
\hline \multirow{2}{*}{ Mixture } & \multirow{2}{*}{$\mathbf{w} / \mathbf{c}$} & \multirow{2}{*}{ Batch } & \multicolumn{6}{|c|}{ Compressive strength (MPa) } & \multirow{2}{*}{$\begin{array}{c}\text { Modulus of } \\
\text { elasticity } \\
\text { (GPa) }\end{array}$} & \multirow{2}{*}{$\begin{array}{c}\text { Poisson's } \\
\text { ratio }\end{array}$} \\
\hline & & & 1d & 3d & $7 d$ & 14d & 28d & 90d & & \\
\hline \multirow{2}{*}{ SL-SF-L1-0.40 } & \multirow{2}{*}{0.4} & 1 & 9.9 & 23.1 & 32.1 & 43.7 & 54.1 & 66.2 & \multirow{2}{*}{34.297} & \multirow{2}{*}{0.23} \\
\hline & & 2 & 9.7 & $-*$ & 31.7 & 43.9 & 53.5 & 65.1 & & \\
\hline \multirow{2}{*}{ FA-SF-L1-0.40 } & \multirow{2}{*}{0.4} & 1 & 15.8 & $-*$ & 38.6 & 47.8 & 53.0 & 67.3 & \multirow{2}{*}{32.524} & \multirow{2}{*}{0.23} \\
\hline & & 2 & 16.2 & 26.9 & 34.3 & 42.6 & 50.0 & 59.6 & & \\
\hline \multirow{2}{*}{ SL-FA-SF-L1-0.40 } & \multirow{2}{*}{0.4} & 1 & 6.5 & 14.1 & 23.2 & 32.4 & 43.1 & 55.7 & \multirow{2}{*}{31.421} & \multirow{2}{*}{0.24} \\
\hline & & 2 & 6.9 & 15.9 & 23.6 & 36.2 & 46.7 & 59.0 & & \\
\hline \multirow{2}{*}{ MK-L1-0.40 } & \multirow{2}{*}{0.4} & 1 & 16.2 & 31.0 & 40.2 & 48.9 & 55.5 & 64.2 & \multirow{2}{*}{34.407} & \multirow{2}{*}{0.22} \\
\hline & & 2 & 17.0 & 33.2 & 42.3 & 51.3 & 58.8 & 70.6 & & \\
\hline \multirow{2}{*}{ SL-SF-L1-0.35 } & \multirow{2}{*}{0.35} & 1 & 12.4 & 25.0 & 36.5 & 48.0 & 62.9 & 72.2 & \multirow{2}{*}{34.234} & \multirow{2}{*}{0.22} \\
\hline & & 2 & 14.6 & 29.4 & 40.1 & 49.4 & 65.1 & 81.0 & & \\
\hline \multirow{2}{*}{ FA-SF-L1-0.35 } & \multirow{2}{*}{0.35} & 1 & 11.5 & 35.1 & 41.3 & 50.0 & 58.2 & 70.6 & \multirow{2}{*}{33.724} & \multirow{2}{*}{0.21} \\
\hline & & 2 & 26.9 & 36.8 & 45.8 & 50.8 & 55.5 & 67.5 & & \\
\hline \multirow{2}{*}{ SL-FA-SF-L1-0.35 } & 0.35 & 1 & 6.0 & 21.4 & 32.7 & 45.6 & 54.6 & 68.1 & 33400 & 033 \\
\hline & 0.35 & 2 & 9.9 & 24.4 & 28.7 & 43.7 & 52.4 & 66.2 & 33.400 & 0.23 \\
\hline & 0.35 & 1 & 18.1 & 37.1 & 49.7 & 60.1 & 71.1 & 78.8 & 33.862 & 0.22 \\
\hline NIN-L1-0.35 & 0.30 & 2 & 17.6 & 36.2 & 48.6 & 61.5 & 71.7 & 81.0 & 33.002 & 0.22 \\
\hline & 03 & 1 & 13.2 & 29.4 & 42.6 & 54.4 & 66.7 & 91.1 & & \\
\hline SL-SF-L1-0.30 & 0.3 & 2 & 10.7 & 32.1 & 44.2 & 56.0 & 69.7 & 88.1 & 34.703 & 0.23 \\
\hline & & 1 & 17.3 & 39.0 & 48.9 & 54.6 & 67.5 & 76.9 & 33628 & \\
\hline FA-SF-LI-0.30 & 0.3 & 2 & 16.1 & 38.2 & 48.0 & 56.6 & 69.5 & 82.9 & 33.628 & 0.22 \\
\hline & & 1 & 12.9 & 28.0 & 39.5 & 57.1 & 68.6 & 82.9 & & \\
\hline SL-FA-SF-L1-U.3U & 0.3 & 2 & 8.0 & 31.0 & 44.5 & 59.9 & 73.9 & 86.2 & 32.262 & 0.23 \\
\hline & & 1 & 17.0 & 51.9 & 67.0 & 79.6 & 92.0 & 102.4 & & \\
\hline MIK-LI-U.30 & 0.3 & 2 & 28.0 & 51.6 & 61.8 & 70.6 & 80.7 & 86.5 & 35.069 & 0.22 \\
\hline
\end{tabular}


(Continued)

\begin{tabular}{|c|c|c|c|c|c|c|c|c|c|c|}
\hline \multirow{2}{*}{ Mixture } & \multirow{2}{*}{$\mathbf{w} / \mathbf{c}$} & \multirow{2}{*}{ Batch } & \multicolumn{6}{|c|}{ Compressive strength(MPa) } & \multirow{2}{*}{$\begin{array}{c}\text { Modulus of } \\
\text { elasticity } \\
\text { (GPa) }\end{array}$} & \multirow{2}{*}{$\begin{array}{c}\text { Poisson's } \\
\text { ratio }\end{array}$} \\
\hline & & & 1d & 3d & 7d & 14d & 28d & 90d & & \\
\hline \multirow{2}{*}{ SL-SF-L2-0.40 } & \multirow[b]{2}{*}{0.4} & 1 & 6.6 & 16.7 & 24.2 & 31.8 & 44.8 & 59.9 & \multirow{2}{*}{31.331} & \multirow{2}{*}{0.23} \\
\hline & & 2 & 12.9 & 29.7 & 40.6 & 53.0 & 66.2 & 73.6 & & \\
\hline \multirow{2}{*}{ FA-SF-L2-0.40 } & \multirow{2}{*}{0.4} & 1 & 10.2 & 24.2 & 36.5 & 39.3 & 48.9 & 59.6 & \multirow{2}{*}{30.434} & \multirow{2}{*}{0.21} \\
\hline & & 2 & 16.5 & 31.8 & 39.0 & 48.9 & 59.6 & 68.6 & & \\
\hline \multirow{2}{*}{ SL-FA-SF-L2-0.40 } & \multirow{2}{*}{0.4} & 1 & 12.4 & 19.5 & 28.0 & 38.2 & 50.0 & 64.0 & \multirow{2}{*}{31.041} & \multirow{2}{*}{0.21} \\
\hline & & 2 & 8.0 & 17.0 & 26.9 & 42.3 & 49.4 & 61.8 & & \\
\hline \multirow{2}{*}{ MK-L2-0.40 } & \multirow{2}{*}{0.4} & 1 & 26.4 & 37.3 & 46.7 & 53.0 & 62.0 & 71.4 & \multirow{2}{*}{33.800} & \multirow{2}{*}{0.20} \\
\hline & & 2 & 16.2 & 29.4 & 42.0 & 52.2 & 64.5 & 71.9 & & \\
\hline \multirow{2}{*}{ SL-SF-G1-0.40 } & \multirow{2}{*}{0.4} & 1 & 11.5 & 24.7 & 29.7 & 40.1 & 48.6 & 59.6 & \multirow{2}{*}{25.552} & \multirow{2}{*}{0.22} \\
\hline & & 2 & 9.3 & 20.0 & 26.1 & 35.4 & 43.4 & 58.5 & & \\
\hline \multirow{2}{*}{ FA-SF-G1-0.40 } & \multirow{2}{*}{0.4} & 1 & 17.0 & 32.4 & 39.0 & 46.4 & 51.3 & 64.5 & \multirow{2}{*}{23.076} & \multirow{2}{*}{0.23} \\
\hline & & 2 & 13.5 & 23.6 & 31.3 & 37.9 & 46.4 & 59.6 & & \\
\hline \multirow{2}{*}{ SL-FA-SF-G1-0.40 } & 04 & 1 & 13.7 & 20.6 & 29.7 & 38.2 & 48.9 & 65.1 & 2017 & כ20 \\
\hline & 0.4 & 2 & 9.1 & 18.4 & 25.0 & 32.9 & 43.4 & 59.9 & 22.911 & 0.22 \\
\hline MK-G1-0 40 & 04 & 1 & 17.6 & 35.7 & 38.7 & 44.5 & 51.9 & 60.4 & 25648 & 023 \\
\hline MK-GI-0.40 & 0.4 & 2 & 18.4 & 34.3 & 42.8 & 51.3 & 56.3 & 63.1 & 25.648 & 0.23 \\
\hline SI SE-C2R 10 & 04 & 1 & 9.6 & 17.6 & 27.7 & 34.9 & 46.9 & 58.2 & 26066 & $0>0$ \\
\hline SL-SF-G2-0.40 & 0.4 & 2 & 13.7 & 23.6 & 34.0 & 40.1 & 51.1 & 59.6 & 26.966 & 0.20 \\
\hline $5 A \mathrm{CE} C 2040$ & 04 & 1 & 13.7 & 20.0 & 27.5 & 34.3 & 43.1 & 48.9 & 25062 & 010 \\
\hline $\mathrm{FA}-\mathrm{SF}-\mathrm{G}<-0.40$ & 0.4 & 2 & 15.9 & 28.0 & 35.7 & 43.7 & 48.3 & 58.2 & 25.802 & 0.19 \\
\hline SL-FA-SF-G $2-040$ & 04 & 1 & 4.7 & 10.2 & 15.4 & 20.6 & 32.7 & 42.0 & 23034 & 020 \\
\hline & 0.4 & 2 & 10.2 & 16.7 & 23.1 & 31.8 & 43.7 & 50.8 & 23.034 & \\
\hline MK-G2-0.40 & 0.4 & 1 & 23.6 & 33.2 & 41.7 & 48.0 & 55.2 & 57.4 & 26.554 & 0.19 \\
\hline
\end{tabular}

Note: 1. * Asterisk, indicates data not considered in this study, because values were outside the expected ranges.

2. $1 \mathrm{MPa}=145 \mathrm{psi}$. 


\section{APPENDIX B}

\section{Direct Tensile Strength at Different Ages of Selected HPC Mixtures}

\begin{tabular}{|c|c|c|c|c|c|c|}
\hline \multirow{2}{*}{ Mixture } & \multirow{2}{*}{ Specimens } & \multicolumn{5}{|c|}{ Direct tensile strength (MPa) } \\
\hline & & 3d & $7 d$ & 14d & 28d & 90d \\
\hline \multirow{4}{*}{ SL-SF-L1-0.4 } & 1 & 2.052 & 2.257 & 2.440 & 2.634 & 2.866 \\
\hline & 2 & 2.033 & 2.223 & 2.671 & 2.597 & $(2.311)$ \\
\hline & 3 & 1.997* & $2.192 *$ & 2.687 & 2.903* & $(2.089)^{*}$ \\
\hline & Ave. & 2.028 & 2.225 & 2.599 & 2.712 & 2.866 \\
\hline \multirow{4}{*}{ FA-SF-L1-0.4 } & 1 & 2.071 & 2.242 & 2.596 & 2.952 & 2.743 \\
\hline & 2 & $(0.983)$ & 2.121 & 2.777 & 2.681 & 2.863 \\
\hline & 3 & $2.045^{*}$ & $2.345^{*}$ & 2.669 & $2.874 *$ & $2.904^{*}$ \\
\hline & Ave. & 2.058 & 2.236 & 2.682 & 2.836 & 2.837 \\
\hline \multirow{4}{*}{ MK-L1-0.4 } & 1 & 1.964 & 2.691 & 2.666 & 3.324 & 2.761 \\
\hline & 2 & 2.420 & 2.590 & 2.903 & 2.531 & 3.322 \\
\hline & 3 & $2.347 *$ & $2.010 *$ & 2.792 & $3.274 *$ & $3.216^{*}$ \\
\hline & Ave. & 2.244 & 2.641 & 2.787 & 3.044 & 3.100 \\
\hline \multirow{4}{*}{ SL-SF-L1-0.35 } & 1 & 2.517 & 3.001 & 2.881 & 3.538 & 3.202 \\
\hline & 2 & 2.356 & 2.566 & 3.456 & 3.148 & 3.559 \\
\hline & 3 & $2.310^{*}$ & 2.969* & 2.929 & 3.494* & $3.282^{*}$ \\
\hline & Ave. & 2.395 & 2.846 & 3.089 & 3.394 & 3.348 \\
\hline \multirow{4}{*}{ FA-SF-L1-0.35 } & 1 & 2.492 & (1.299) & 2.712 & 3.528 & 3.381 \\
\hline & 2 & 2.508 & 2.782 & 3.457 & 3.163 & 3.162 \\
\hline & 3 & $2.422 *$ & $2.720 *$ & 3.147 & 3.381* & $3.042 *$ \\
\hline & Ave. & 2.475 & 2.752 & 3.106 & 3.358 & 3.195 \\
\hline \multirow{4}{*}{ MK-L1-0.35 } & 1 & 2.887 & 2.898 & 2.934 & 3.871 & 3.461 \\
\hline & 2 & 2.353 & 2.834 & 3.542 & 2.824 & 2.848 \\
\hline & 3 & $2.321^{*}$ & $2.775 *$ & 2.845 & 3.499* & $3.356^{*}$ \\
\hline & Ave. & 2.521 & 2.836 & 3.238 & 3.399 & 3.222 \\
\hline \multirow{4}{*}{ SL-SF-L1-0.30 } & 1 & (2.116) & 2.751 & 3.087 & 3.544 & 3.187 \\
\hline & 2 & 2.937 & 3.364 & 3.223 & 3.501 & 3.581 \\
\hline & 3 & $2.799 *$ & $2.851^{*}$ & 3.294 & $3.443^{*}$ & $3.259 *$ \\
\hline & Ave. & 2.869 & 2.989 & 3.202 & 3.497 & 3.343 \\
\hline \multirow{4}{*}{ FA-SF-L1-0.30 } & 1 & 2.883 & $(2.226)$ & 3.269 & 3.549 & 3.201 \\
\hline & 2 & 2.803 & 2.967 & 3.454 & 3.464 & 3.346 \\
\hline & 3 & $2.808 *$ & 2.853* & $(2.368)$ & 3.355* & $2.878^{*}$ \\
\hline & Ave. & 2.832 & 2.911 & 3.362 & 3.457 & 3.274 \\
\hline \multirow{4}{*}{ MK-L1-0.30 } & 1 & 3.069 & 3.158 & 3.269 & $(2.580)$ & 3.274 \\
\hline & 2 & 2.764 & 3.058 & $(2.428)$ & 3.599 & $(2.541)$ \\
\hline & 3 & $2.862^{*}$ & $3.020^{*}$ & 3.516 & $3.501^{*}$ & 3.467* \\
\hline & Ave. & 2.899 & 3.079 & 3.393 & 3.550 & 3.371 \\
\hline
\end{tabular}

(To be continued) 
(Continued)

\begin{tabular}{|c|c|c|c|c|c|c|}
\hline \multirow{2}{*}{ Mixture } & \multirow{2}{*}{ Specimens } & \multicolumn{5}{|c|}{ Direct tensile strength (MPa) } \\
\hline & & $3 d$ & $7 d$ & 14d & 28d & 90d \\
\hline \multirow{4}{*}{ SL-SF-L2-0.4 } & 1 & 1.955 & 2.658 & 2.890 & 2.840 & 2.939 \\
\hline & 2 & 1.973 & 2.748 & 2.869 & 3.057 & 2.946 \\
\hline & 3 & $2.013^{*}$ & $2.639 *$ & 2.823 & $2.870^{*}$ & $2.935^{*}$ \\
\hline & Ave. & 1.981 & 2.682 & 2.861 & 2.949 & 2.941 \\
\hline \multirow{4}{*}{ FA-SF-L2-0.4 } & 1 & 1.720 & 2.680 & $(2.263)$ & 2.949 & 2.931 \\
\hline & 2 & 1.900 & $(2.148)$ & 2.894 & 2.900 & 2.880 \\
\hline & 3 & $1.920 *$ & $2.460 *$ & 2.868 & $2.884 *$ & $2.766^{*}$ \\
\hline & Ave. & 1.847 & 2.570 & 2.882 & 2.911 & 2.859 \\
\hline \multirow{4}{*}{ MK-L2-0.4 } & 1 & 2.154 & 2.832 & 2.870 & 2.873 & 3.017 \\
\hline & 2 & 2.190 & 2.765 & 2.982 & 3.439 & 3.004 \\
\hline & 3 & $2.275^{*}$ & 2.754* & 3.237 & 3.167* & $3.126 *$ \\
\hline & Ave. & 2.207 & 2.784 & 2.927 & 3.160 & 3.050 \\
\hline \multirow{4}{*}{ SL-SF-G1-0.4 } & 1 & 1.967 & 2.040 & (1.696) & 2.731 & 2.898 \\
\hline & 2 & 1.815 & 2.102 & 2.462 & 2.844 & (2.373) \\
\hline & 3 & $1.660^{*}$ & $2.035 *$ & 2.296 & $(2.151)^{*}$ & $2.886^{*}$ \\
\hline & Ave. & 1.814 & 2.060 & 2.380 & 2.788 & 2.893 \\
\hline \multirow{4}{*}{ FA-SF-G1-0.4 } & 1 & $(0.946)$ & 2.228 & 2.413 & 2.817 & (2.305) \\
\hline & 2 & 2.047 & 2.182 & 2.444 & 2.692 & 2.858 \\
\hline & 3 & $1.440 *$ & 2.096* & 2.550 & $2.612 *$ & $2.674 *$ \\
\hline & Ave. & 1.744 & 2.169 & 2.470 & 2.707 & 2.766 \\
\hline \multirow{4}{*}{ MK-G1-0.4 } & 1 & 2.220 & 2.559 & 2.630 & (2.237) & 2.869 \\
\hline & 2 & 2.025 & 2.570 & 2.648 & 2.917 & 2.925 \\
\hline & 3 & 2.033* & $2.486 *$ & (2.093) & 2.799* & $2.844 *$ \\
\hline & Ave. & 2.093 & 2.538 & 2.640 & 2.858 & 2.880 \\
\hline \multirow{4}{*}{ SL-SF-G2-0.4 } & 1 & 2.012 & 2.376 & 2.745 & 2.893 & 2.907 \\
\hline & 2 & 1.803 & 2.372 & 2.504 & 2.930 & $(2.235)$ \\
\hline & 3 & 1.875* & $2.427 *$ & 2.673 & 2.796* & $2.852 *$ \\
\hline & Ave. & 1.897 & 2.392 & 2.641 & 2.873 & 2.880 \\
\hline \multirow{4}{*}{ FA-SF-G2-0.4 } & 1 & 1.947 & 2.028 & 2.299 & 2.444 & 2.555 \\
\hline & 2 & $(1.032)$ & 2.066 & 2.156 & 2.409 & 2.632 \\
\hline & 3 & $1.476^{*}$ & $2.018 *$ & 2.244 & $2.640 *$ & (2.119)* \\
\hline & Ave. & 1.712 & 2.038 & 2.233 & 2.498 & 2.594 \\
\hline \multirow{4}{*}{ MK-G2-0.4 } & 1 & 2.235 & 2.698 & 2.725 & 3.138 & 2.785 \\
\hline & 2 & 2.289 & 2.372 & 2.729 & 2.778 & 2.882 \\
\hline & 3 & $2.152^{*}$ & $2.450^{*}$ & 2.787 & $2.905^{*}$ & 2.993* \\
\hline & Ave. & 2.226 & 2.507 & 2.728 & 2.941 & 2.887 \\
\hline
\end{tabular}

Note:

1. * Asterisk, indicates data collected for instrumented samples with strain gages, to compute tensile modulus of elasticity.

2. ${ }^{(}$Parenthesis, indicates data not considered in this study, because values were outside the expected ranges. In most cases one out of three was discarded, except for 90-day SL-SF-L1-0.4.

3. $1 \mathrm{MPa}=145 \mathrm{psi}$ 


\section{APPENDIX C}

\section{Free Shrinkage with Time for Selected HPC Mixtures}

\begin{tabular}{|c|c|c|c|c|c|c|c|c|c|c|c|c|c|c|c|c|c|c|c|}
\hline \multirow{2}{*}{ Mixtures } & \multicolumn{19}{|c|}{ Free shrinkage $(\mu \varepsilon)$} \\
\hline & $\mathbf{0}$ & 1 & 2 & 3 & 4 & 5 & 6 & 7 & 10 & 14 & 17 & 23 & 28 & 30 & 40 & 50 & 60 & 70 & 90 \\
\hline SL-SF-L1-0.4 & 0 & 42 & 98 & 133 & 196 & 245 & 275 & 298 & 345 & 385 & 417 & 441 & 455 & 466 & 490 & 508 & 517 & 527 & 550 \\
\hline FA-SF-L1-0.4 & 0 & 58 & 98 & 129 & 163 & 198 & 228 & 259 & 310 & 352 & 385 & 406 & 424 & 436 & 473 & 494 & 508 & 524 & 541 \\
\hline MK-L1-0.4 & 0 & 47 & 91 & 149 & 179 & 210 & 235 & 256 & 282 & 310 & 343 & 378 & 408 & 417 & 445 & 462 & 476 & 494 & 517 \\
\hline SL-SF-L1-0.35 & 0 & 103 & 170 & 221 & 266 & 301 & 331 & 350 & 389 & 427 & 459 & 499 & 524 & 531 & 552 & 569 & 585 & 599 & 627 \\
\hline FA-SF-L1-0.35 & 0 & 112 & 177 & 219 & 280 & 322 & 359 & 396 & 431 & 462 & 494 & 531 & 555 & 571 & 597 & 611 & 625 & 639 & 650 \\
\hline MK-L1-0.35 & 0 & 142 & 191 & 235 & 277 & 310 & 333 & 352 & 380 & 408 & 441 & 466 & 490 & 499 & 524 & 543 & 557 & 569 & 592 \\
\hline SL-SF-L1-0.3 & 0 & 100 & 175 & 240 & 289 & 333 & 371 & 396 & 445 & 490 & 531 & 571 & 604 & 611 & 636 & 664 & 685 & 695 & 716 \\
\hline FA-SF-L1-0.3 & 0 & 119 & 189 & 233 & 270 & 308 & 343 & 373 & 427 & 471 & 510 & 538 & 559 & 576 & 604 & 627 & 648 & 681 & 702 \\
\hline MK-L1-0.3 & 0 & 117 & 198 & 259 & 303 & 340 & 378 & 399 & 438 & 478 & 506 & 529 & 552 & 562 & 590 & 606 & 618 & 625 & 648 \\
\hline SL-SF-L2-0.4 & 0 & 51 & 91 & 147 & 187 & 213 & 240 & 272 & 301 & 333 & 360 & 397 & 419 & 427 & 445 & 467 & 488 & 509 & 539 \\
\hline FA-SF-L2-0.4 & 0 & 32 & 77 & 131 & 157 & 189 & 213 & 245 & 288 & 341 & 365 & 403 & 435 & 445 & 464 & 488 & 504 & 520 & 549 \\
\hline MK-L2-0.4 & 0 & 77 & 120 & 165 & 213 & 240 & 256 & 275 & 304 & 341 & 360 & 389 & 421 & 435 & 445 & 469 & 485 & 496 & 517 \\
\hline SL-SF-G1-0.4 & 0 & 47 & 100 & 140 & 170 & 205 & 228 & 252 & 289 & 312 & 338 & 373 & 401 & 424 & 455 & 471 & 485 & 501 & 524 \\
\hline FA-SF-G1-0.4 & 0 & 47 & 103 & 131 & 163 & 186 & 219 & 242 & 284 & 324 & 347 & 387 & 408 & 417 & 438 & 459 & 476 & 492 & 520 \\
\hline MK-G1-0.4 & 0 & 52 & 112 & 140 & 164 & 203 & 227 & 262 & 304 & 322 & 343 & 381 & 409 & 420 & 441 & 458 & 469 & 483 & 493 \\
\hline SL-SF-G2-0.4 & 0 & 47 & 100 & 140 & 170 & 205 & 228 & 252 & 289 & 312 & 338 & 373 & 401 & 424 & 455 & 471 & 485 & 501 & 524 \\
\hline FA-SF-G2-0.4 & 0 & 47 & 103 & 131 & 163 & 186 & 219 & 242 & 284 & 324 & 347 & 387 & 408 & 417 & 438 & 459 & 476 & 492 & 520 \\
\hline MK-G2-0.4 & 0 & 52 & 112 & 140 & 164 & 203 & 227 & 262 & 304 & 322 & 343 & 381 & 409 & 420 & 441 & 458 & 469 & 483 & 493 \\
\hline
\end{tabular}


Moist Cuing Correction Factor $\gamma_{c p}$ in ACI 209R

\begin{tabular}{c|c}
\hline $\begin{array}{c}\text { Moist curing duration } \\
\text { (days) }\end{array}$ & $\gamma_{c p}$ \\
\hline 1 & 1.2 \\
\hline 3 & 1.1 \\
\hline 7 & 1.0 \\
\hline 14 & 0.93 \\
\hline 28 & 0.86 \\
\hline 90 & 0.75 \\
\hline
\end{tabular}

(Adopted from: ACI 209 R, 1992) 


\section{APPENDIX D}

function $[\mathrm{a}, \mathrm{R}]=\mathrm{R}$-curve (p, cmod, $\mathrm{E}$ )

$\%$ Function to calculate R-curve

$\% \mathrm{p}$ is load, and CMOD is corresponding crack mouth opening displacement

$\% \mathrm{E}$ is tensile modulus of elasticity

$L=483 ; S=406 ; b=101 ; w=0.231 ; t=101 ; \%$ basic information of the beam

lh =length(p); \% length of the load matrix

for $\mathrm{i}=1: 1: \mathrm{lh}$

$\mathrm{M}=\mathrm{p}(\mathrm{i}) * \mathrm{~S} / 4+\mathrm{w}^{*} \mathrm{~L} *(2 * \mathrm{~S}-\mathrm{L}) / 8 ; \%$ calculate the moment at notch section

$\mathrm{A}=\mathrm{cmod}(\mathrm{i}) * \mathrm{E} * \mathrm{~b} * \mathrm{t} / \mathrm{M}$;

$\mathrm{A} 1=-48.96 ; \mathrm{A} 2=190.8 ; \mathrm{A} 3=-298.44 ; \mathrm{A} 4=220.56$;

$\mathrm{A} 5=-(91.68+\mathrm{A}) ; \mathrm{A} 6=30.08+2 * \mathrm{~A} ; \mathrm{A} 7=-\mathrm{A}$;

$\%$ constant of the 6 th order polynomial function

$\mathrm{c}=$ [A1 A2 A3 A4 A5 A6 A7] \% to solve the polynomial function

$\mathrm{r}=\operatorname{roots}(\mathrm{c})$;

alpha $=\mathrm{r}(6) ; \quad \%$ alpha is the notch to depth ratio(a/b)

$\mathrm{a}(\mathrm{i})=\mathrm{b}$ *alpha; $\quad \%$ crack extension length

$\mathrm{KI}(\mathrm{i})=6 * \mathrm{M} * \operatorname{sqrt}(\mathrm{a}(\mathrm{i})) /(\mathrm{b} * \mathrm{~b} * \mathrm{t}) *((1.99-$ alpha*(1-alpha)*(2.15-

3.93*alpha+2.7*alpha^2) $) /((1+2 *$ alpha $) *(1$-alpha $) \wedge(3 / 2)))$;

$\%$ calculate the stress intensity factor

$\mathrm{R}(\mathrm{i})=1000 * \mathrm{KI}(\mathrm{i}) \wedge 2 / \mathrm{E} ; \quad \% \mathrm{R}$ curve for ring specimen

End 
function $[\mathrm{ac}, \mathrm{Gc}]=$ findfun $(\mathrm{R}, \mathrm{a}, \mathrm{E})$

$\%$ function to find the critical crack length

$\% \mathrm{R}$ is the R-curve, a is the crack extension length, $\mathrm{E}$ is modulus of elasticity.

$\mathrm{b}=101 ; \quad \%$ depth of the beam

Max=150; \% Assumed the maximum strain for ring specimen

lh=length(a); \% length of crack extension matrix

for $\mathrm{j}=2: \mathrm{lh}-1$

$r(j)=(R(j+1)-R(j-1)) /(a(j+1)-a(j-1))$

end

$\%$ calculate the first order derivative of R-curve

for sigma $=1: 0.5:$ Max $\%$ sigma is the inducted strain inside the concrete ring $\mathrm{i}=2 ; \mathrm{L}=\mathrm{lh}-1$;

while $\mathrm{i}<=\mathrm{L}$

alpha(i)=a(i)/b; \% notch to depth ratio

$\mathrm{F}(\mathrm{i})=5.244 *$ alpha(i)^4-11.431*alpha(i)^3+10.531*alpha(i)^2-2.137*alpha(i)+1.655;

$\%$ geometric factor for G-curve

$\mathrm{G}(\mathrm{i})=0.6 * \mathrm{pi}^{*} \mathrm{E}^{*} \operatorname{sigma}^{\wedge} 2^{*} \mathrm{a}(\mathrm{i})^{*} \mathrm{~F}(\mathrm{i})^{\wedge} 2 ; \quad \%$ calculate G-curve

$\mathrm{f}(\mathrm{i})=20.976 *$ alpha(i)^4-34.29*alpha(i)^3+21.062*alpha(i)^2-2.137*alpha(i) $\quad \%$ geometric factor for first order derivative of G-curve

$\mathrm{g}(\mathrm{i})=0.6 * \mathrm{pi}^{*} \mathrm{E} * \operatorname{sigma} \wedge 2 * \mathrm{~F}(\mathrm{i}) \wedge 2+1.2 * \mathrm{pi}^{*} \mathrm{E} * \operatorname{sigma} \wedge 2 * \mathrm{~F}(\mathrm{i}) * \mathrm{f}(\mathrm{i})$

$\%$ calculate first order derivative of G-curve

if $\mathrm{G}(\mathrm{i})=\mathrm{R}(\mathrm{i})$ and $\mathrm{g}(\mathrm{i})=\mathrm{r}(\mathrm{i}) \quad \%$ the conditions for critical cracking propagation

$\mathrm{ac}=\mathrm{a}(\mathrm{i}) ; \quad \%$ critical crack length

$\mathrm{Gc}=\mathrm{G}(\mathrm{i}) ; \quad \%$ critical fracture energy

break

else $\mathrm{i}=\mathrm{i}+1$

end

end

end 


\section{APPENDIX E}

Creep Compliance $J\left(t, t^{\prime}\right)$ for HPC Mixture SL-SF-L1-04

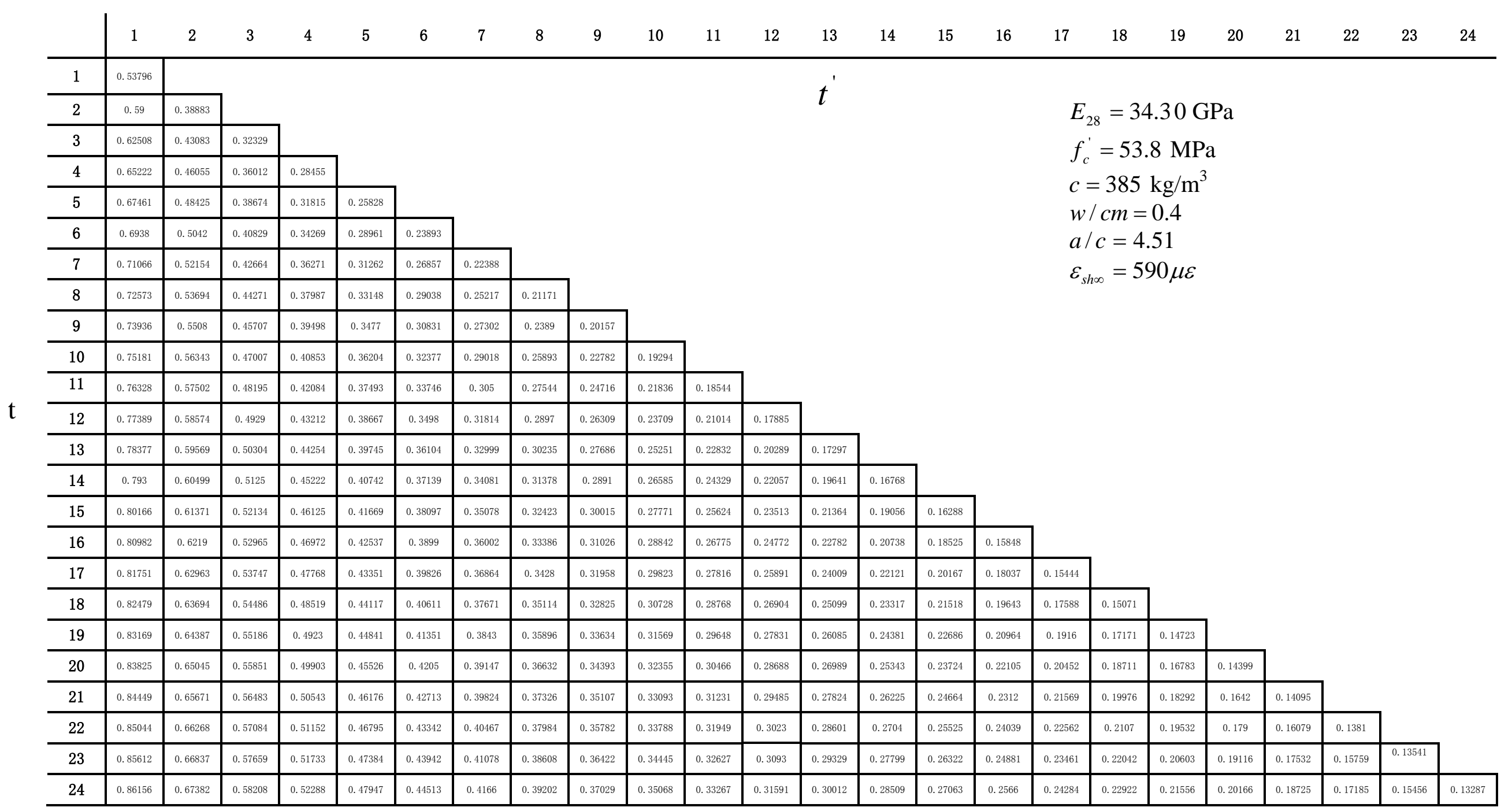




\section{Creep Compliance $J\left(t, t^{\prime}\right)$ for HPC Mixture SL-SF-G2-04}

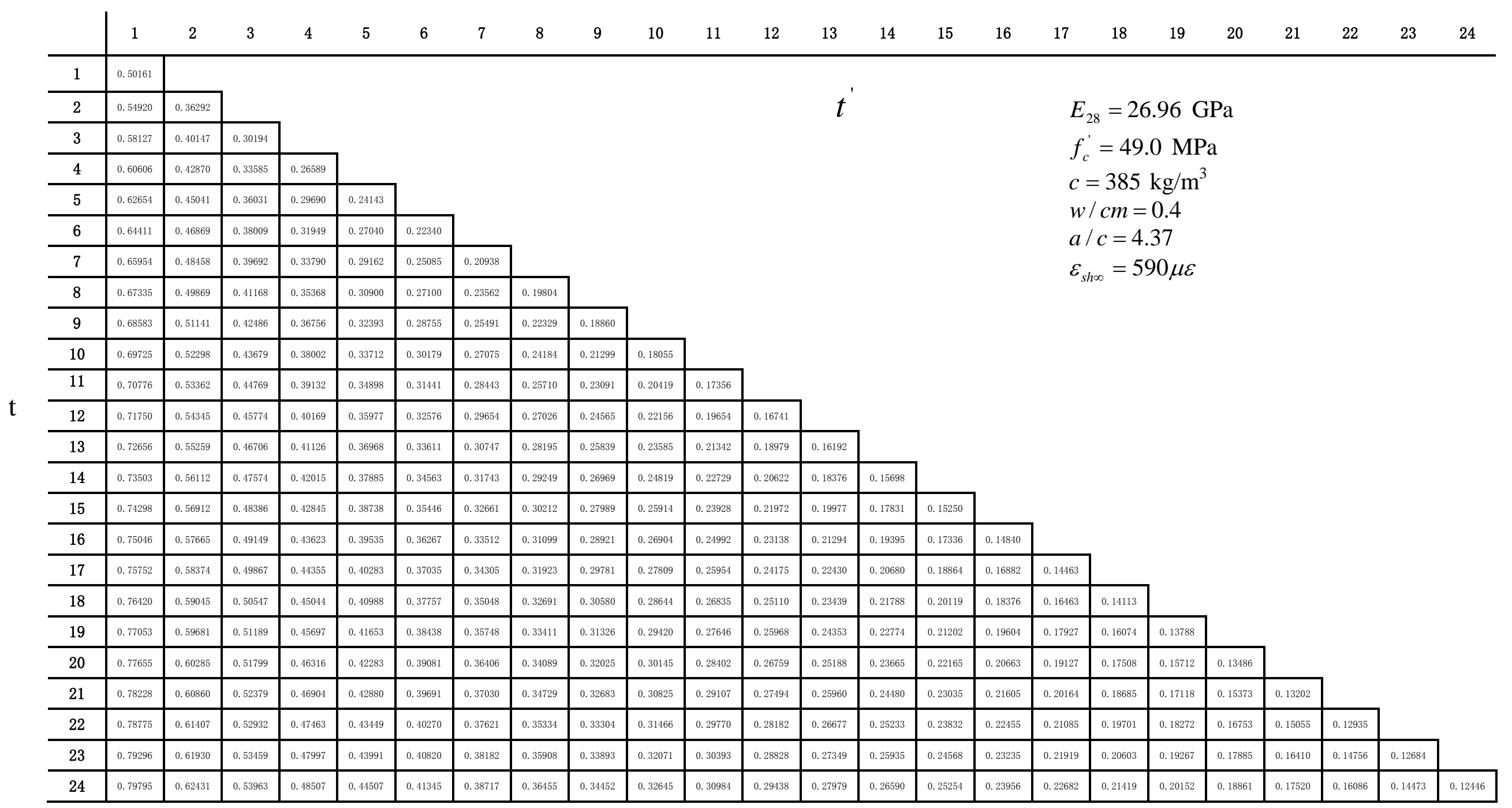




\section{Creep Compliance $J\left(t, t^{\prime}\right)$ for HPC Mixture SL-SF-L1-03}

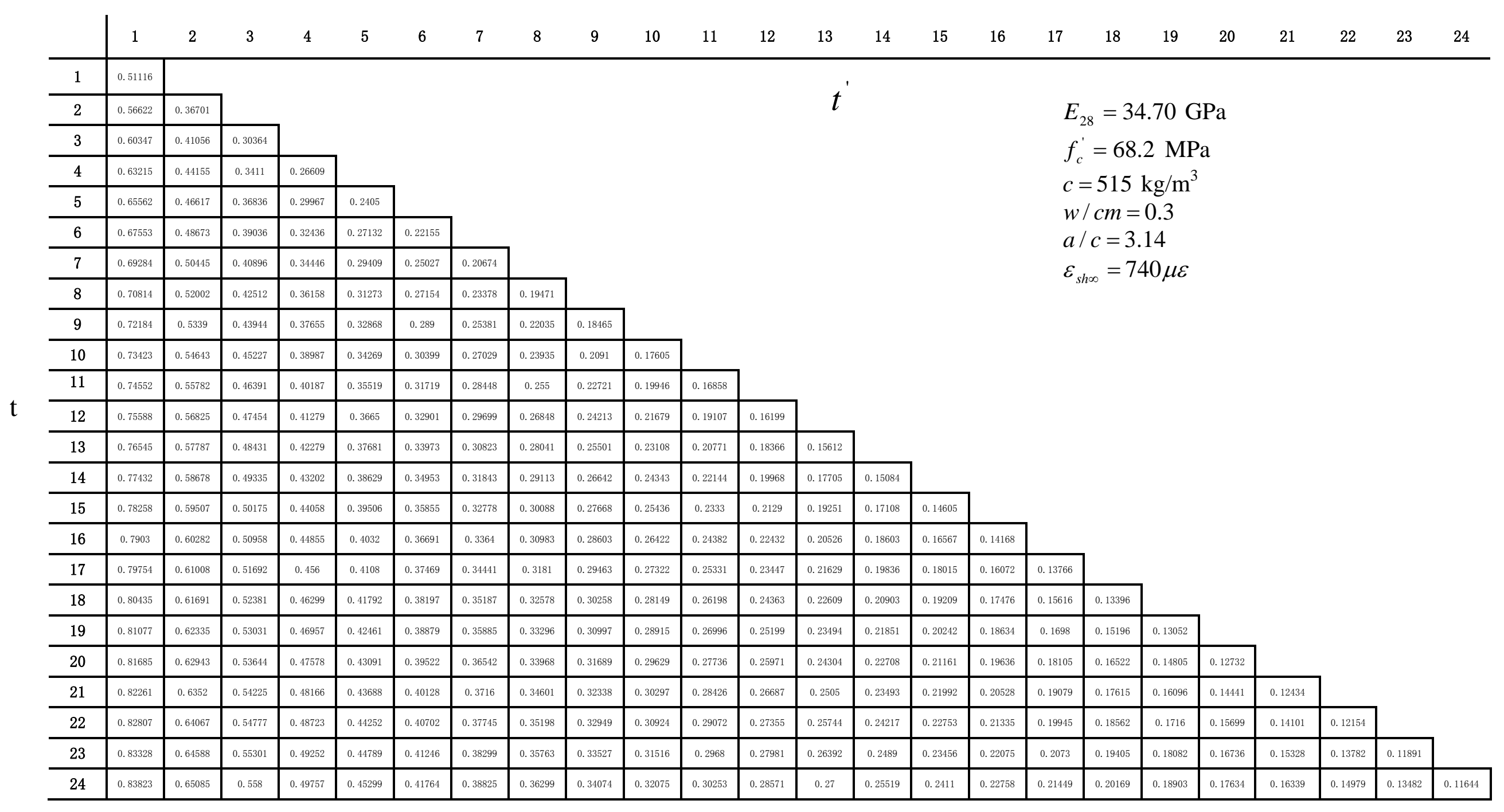

